\title{
Atmospheric Neutrinos In The MINOS Far Detector
}

Caius Leo Frederick Howcroft

of

Peterhouse, Cambridge

A dissertation submitted to the University of Cambridge for the degree of Doctor of Philosophy

8 December 2004 



\title{
Atmospheric Neutrinos In The MINOS Far Detector
}

\author{
Caius Leo Frederick Howcroft
}

\begin{abstract}
The phenomenon of flavour oscillations of neutrinos created in the atmosphere was first reported by the Super-Kamiokande collaboration in 1998 and since then has been confirmed by Soudan 2 and MACRO.

The MINOS Far Detector is the first magnetised neutrino detector able to study atmospheric neutrino oscillations. Although it was designed to detect neutrinos from the NuMI beam, it provides a unique opportunity to measure the oscillation parameters for neutrinos and anti-neutrinos independently. The MINOS Far Detector was completed in August 2003 and since then has collected 2.52 kton-years of atmospheric data.

Atmospheric neutrino interactions contained within the volume of the detector are separated from the dominant background from cosmic ray muons. Thirty seven events are selected with an estimated background contamination of less than $10 \%$. Using the detector's magnetic field, 17 neutrino events and 6 anti-neutrino events are identified, 14 events have ambiguous charge.

The neutrino oscillation parameters for $\nu_{\mu}$ and $\bar{\nu}_{\mu}$ are studied using a maximum likelihood analysis. The measurement does not place constraining limits on the neutrino oscillation parameters due to the limited statistics of the data set analysed. However, this thesis represents the first observation of charge separated atmospheric neutrino interactions. It also details the techniques developed to perform atmospheric neutrino analyses in the MINOS Far Detector.
\end{abstract}




\section{Declaration}

This dissertation is my own work, except where explicit reference is made to the work of others, and has not been submitted for another qualification to this or any other university. This dissertation does not exceed the word limit for the respective Degree Committee.

Caius Howcroft 


\section{Acknowledgments}

One and half thousand pints, two hundred curries and one thesis after I started this whole fieasco there are an enormous number of people to whom I will always be indebted.

First and foremost must come my parents, Sarah Howcroft and Steve Eaton. Without their support I would have never made it to this point. I would also like to thank John Mansfield for his continued friendship over the years.

Mark Thomson, my supervisor at Cambridge, can count the completion of this thesis as a personal achievement. Without his (very) patient guidance I would have never finished it and would have certainly not had so much fun (or beer) along the way. While Mark can claim most of the credit for the completion of frustrating task of steering me through the academic requirements of the last four years, there are other people I must thank. Pat and David Ward have both provided invaluable advise and working in the same group as them has been a joy. Roy Lee who spent the time to show me the ropes of the analysis. Andy Blake for the mammoth contribution he has made to the MINOS atmospheric analysis and his friendship over the last 3 years. Matt Palmer, fellow software victim. Geoff Pearce, Tim Nichols, Tass Belias, Gordon Crone, Nathaniel Tagg and Liza Falk-Harris for all the shared pain over the last 4 years. I must also thank all the members of the MINOS collaboration whos' dedication to the project made this possible and PPRAC for funding my studentship.

Lastly, but by no means least, come the friends I have made at Cambridge and elsewhere, who where there when it counted and without whom the last four years would have intolerable; Piers "Beastie" Baker-Bates, Ed Cambell, Jim Dale, Hillary Dixon, Jeremy Gogos, Catherine "Sequel" Howell, Nico "The Kid" Jose Rodriguez, Katey Lancaster, Tim Langdon, Marta de Magalhae, Jeremy Marchant, Phil Marshall, Adrian Pabst, Rachel "Sparkie" Parker, Mitesh Patel, Dipu Rahman, Richard Roberts, Ben Speakman, Richard J Thomas, Anze Slozar, Emma Toogood, Ross Williamson and Kris Zarb-Admai, may God have mercy on our livers. 
'Thus I descended from the first circle down into the second, which girdles less space, and so much more woe that it goads to wailing. There abides Minos horribly, and snarls; he examines the sins at the entrance; he judges, and he sends according as he entwines himself.'

Dante Alighieri - Divina Commedia. 


\section{Contents}

$\begin{array}{lll}1 & \text { Introduction } & 1\end{array}$

2 Neutrino Oscillations 4

$2.1 \quad$ Neutrino Oscillations In Vacuum . . . . . . . . . . . . . . . . . . 4

2.2 The Chooz Experiment $\ldots \ldots \ldots \ldots \ldots$

2.3 The Solar Neutrino Problem. . . . . . . . . . . . . . . . . . . . 7

2.3 .1 Radiochemical Experiments . . . . . . . . . . . . . . . . 10

$2.3 .2 \quad$ Water Čerenkov Detectors . . . . . . . . . . . . . . . . . . . 11

2.4 KamLAND . . . . . . . . . . . . . . . . . . . . . . . . . 14

2.5 Atmospheric Neutrinos . . . . . . . . . . . . . . . . . . . . 17

2.5 .1 General Features of Atmospheric Neutrino Experiments. . . . . 18

$2.5 .2 \quad$ Atmospheric Neutrino Experiments. . . . . . . . . . . . . . 19

2.6 Long Baseline Accelerator Experiments . . . . . . . . . . . . . . . . 21

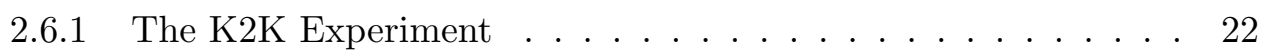

2.6 .2 MINOS . . . . . . . . . . . . . . . . . . . 23

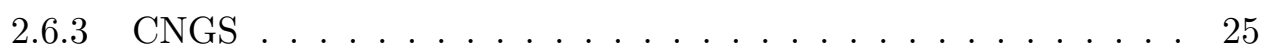

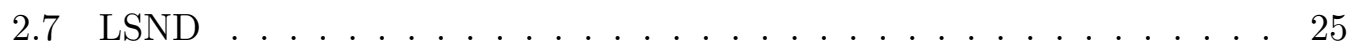

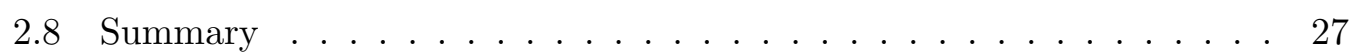

\begin{tabular}{lll}
\hline & The MINOS Far Detector & $\mathbf{2 8}$
\end{tabular}

3.1 Detector Physical Overview . . . . . . . . . . . . . . . . . 28

3.2 Far Detector Front-End Electronics . . . . . . . . . . . . . . . . . . 30

3.3 Event Information . . . . . . . . . . . . . . . . . . . . . . . . 32

3.4 Calibration Overview . . . . . . . . . . . . . . . . . . . . . . . 32

3.4 .1 Energy Calibration . . . . . . . . . . . . . . . . . 32

3.4 .2 Timing Calibration . . . . . . . . . . . . . . . . . . . . . 34

3.5 The Veto Shield $\ldots \ldots \ldots \ldots$. . . . . . . . . . . . . . . . 35 
3.5 .1 Veto Shield Overview. . . . . . . . . . . . . . . . . . . . 35

3.5 .2 Shield Noise . . . . . . . . . . . . . . . . . . . . . 36

3.5 .3 Tuning the PMT Gains . . . . . . . . . . . . . . . . . . . . . . . . . 37

3.5.4 Shield Timing . . . . . . . . . . . . . . . . . . . . . . . . 38

3.6 Summary $\ldots \ldots \ldots \ldots \ldots \ldots$. . . . . . . . . . . . . . . . . 41

\begin{tabular}{|lll|}
\hline 4 & The MINOS Data Acquisition System & 42
\end{tabular}

$4.1 \quad$ Far Detector Data Acquisition System Operation . . . . . . . . . . . . 42

4.1 .1 The Trigger Farm . . . . . . . . . . . . . . . . . . . 44

4.1 .2 Data Collection Process . . . . . . . . . . . . . . . . . 45

4.2 Run Control . . . . . . . . . . . . . . . . . . . . . . . . . . . . . . . 47

$4.2 .1 \quad$ Design and Implementation . . . . . . . . . . . . . . . . . 48

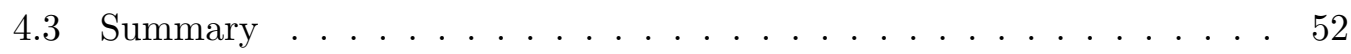

5 Monte Carlo Simulation 5

5.1 Monte Carlo . . . . . . . . . . . . . . . . . . . . . . 53

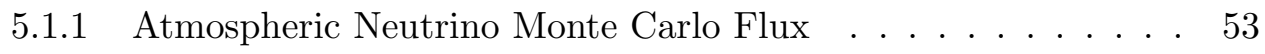

5.1 .2 General Features Of Atmospheric Fluxes . . . . . . . . . . . 57

5.1 .3 Neutrino Cross-Sections . . . . . . . . . . . . . . . 58

5.1 .4 Systematic Uncertainties on Number of Neutrino Interactions . 60

5.1 .5 Cosmic Ray Muon Monte Carlo Flux. . . . . . . . . . . . . . 62

5.1 .6 Particle Tracking and Detector Simulation . . . . . . . . . . . 64

5.1 .7 Monte Carlo Production . . . . . . . . . . . . . . . . . . . 64

$5.2 \quad$ Data and Monte Carlo Comparison . . . . . . . . . . . . . . . 64

$5.2 .1 \quad$ Event Timing . . . . . . . . . . . . . . . . . . 65

5.2 .2 Strip Response and Energy Deposition . . . . . . . . . . . . 65

5.2 .3 Charge Reconstruction . . . . . . . . . . . . . . 67

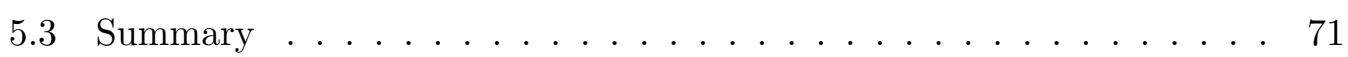

\begin{tabular}{lll}
\hline 6 & Signal Selection & $\mathbf{7 2}$
\end{tabular}

6.1 Data Selection . . . . . . . . . . . . . . . . . . 72

6.2 Event Reconstruction $\ldots \ldots \ldots \ldots$. . . . . . . . . . . 73

6.3 Background Rejection . . . . . . . . . . . . . . . . . . . . . 74

6.3 .1 Track Length . . . . . . . . . . . . . . . . . . . . . . . . . . . . . . . . . . . .

6.3 .2 Containment . . . . . . . . . . . . . . . 75

6.3 .3 Track Quality . . . . . . . . . . . . . . . . . . . 75

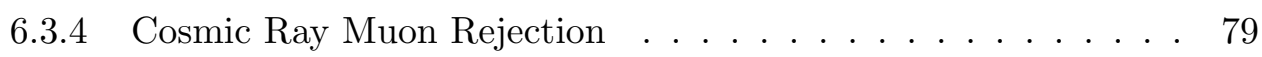


6.3 .5 Event Quality . . . . . . . . . . . . . . . . . . . . 86

6.4 The MINOS Veto Shield . . . . . . . . . . . . . . . . . . . . . . . . . 87

$6.4 .1 \quad$ Background Rejection Efficiency . . . . . . . . . . . . . 88

$6.4 .2 \quad$ Signal Inefficiencies . . . . . . . . . . . . . . . . . . . . . . 89

6.4 .3 Using the Veto Shield . . . . . . . . . . . . . . . . . . . 91

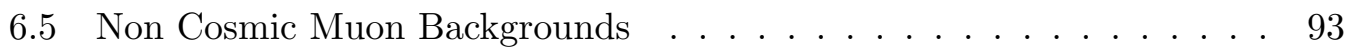

$6.5 .1 \quad$ Neutron Backgrounds $\ldots \ldots \ldots \ldots$. . . . . . . . . . 93

6.5 .2 Neutrino Backgrounds . . . . . . . . . . . . . . . . . 93

6.6 Signal Efficiencies . . . . . . . . . . . . . . . . . . . . . . . . . . 95

6.7 Selected Events . . . . . . . . . . . . . . . . . . . . . . 95

6.8 Summary . . . . . . . . . . . . . . . . . . . . . . . . 101

\begin{tabular}{lll}
\hline 7 & Neutrino Oscillation Analysis & 102
\end{tabular}

7.1 Event Properties . . . . . . . . . . . . . . . . . 102

$7.1 .1 \quad$ Event Direction . . . . . . . . . . . . . . . . . . . . . 102

7.1 .2 Energy Reconstruction . . . . . . . . . . . . . . . . . . . 109

$7.1 .3 \quad$ Charge Reconstruction . . . . . . . . . . . . . . . . 112

7.2 Neutrino Flux Charge Ratio . . . . . . . . . . . . . . . . . . . . 117

7.3 Methods of Oscillation Parameter Estimation . . . . . . . . . . . 118

7.4 Discussion of Systematics $\ldots \ldots$. . . . . . . . . . . . . 125

7.4 .1 Normalisation . . . . . . . . . . . . . . . . . . 125

7.4 .2 Hadronic Models . . . . . . . . . . . . . . . . . . 126

7.4 .3 Event Timing . . . . . . . . . . . . . . . . . . . . . . . . 129

7.4 .4 Neutrino Cross Sections . . . . . . . . . . . . . . . . . . . . . . 129

7.5 Expected 25 kton-year Sensitivity . . . . . . . . . . . . . . 130

7.6 Fit to Data . . . . . . . . . . . . . . . . . . . . . . . . . . 131

7.7 Charge Separated Oscillation Measurement . . . . . . . . . . . . 137

7.8 Summary . . . . . . . . . . . . . . . . . . . . . . . . . 140

\begin{tabular}{lll}
\hline 8 & Discussion & 141
\end{tabular}

\begin{tabular}{lr}
\hline A Selected Events & 143
\end{tabular} 


\section{List of Figures}

2.1 The Chooz exclusion plot. . . . . . . . . . . . . . . . . 8

2.2 The Standard Solar Model neutrino flux. . . . . . . . . . . . . . . . . . 9

2.3 The angle between the observed electron and the sun for Super-Kamiokande solar neutrino experiment. . . . . . . . . . . . . . . . . . . 11

2.4 The allowed regions of solar parameter space after the Super-Kamiokande results. . . . . . . . . . . . . . . . . . . 13

2.5 The flux of $\nu_{e}$ and $\nu_{\mu, \tau}$ as measured by the SNO experiment. . . . . . 14

2.6 The allowed regions for $\Delta m_{12}^{2}$ and $\sin ^{2} 2 \theta$ obtained by combining KamLAND and solar neutrino results. . . . . . . . . . . . . . . . 15

2.7 The prompt event energy spectrum measured by KamLAND . . . . 16

2.8 Sketch of the production of atmospheric neutrinos $\ldots \ldots \ldots . \ldots 17$

2.9 The atmospheric $\nu_{\mu}$ survival probability as a function of cosine of the zenith angle and the neutrino energy for four different values of $\Delta m_{23}^{2}$ assuming maximal mixing. . . . . . . . . . . . . . . . . 18

2.10 The zenith angle distributions for atmospheric neutrinos in SuperKamiokande ...................... 20

2.11 The allowed regions for $\nu_{\mu} \leftrightarrow \nu_{\tau}$ atmospheric neutrino oscillations. . . 21

$2.12 L / E$ distributions for electron and muon type events from the Soudan

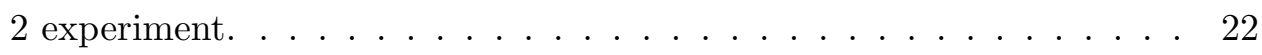

2.13 The neutrino energy spectrum measured by K2K experiment. . . . . 24

2.14 The allowed regions obtained by the K2K experiment. . . . . . . . . 25

2.15 The expected sensitivity of the MINOS beam analysis. . . . . . . . . 26

3.1 A schematic of the MINOS Far Detector. . . . . . . . . . . . . . . 29

$3.2 \quad$ Photograph of the completed MINOS Far Detector. . . . . . . . . . . 30

3.3 An example of a multiplexed event. . . . . . . . . . . . . . . . 31

$3.4 \quad$ An event display of an example cosmic ray muon event in the MINOS Far Detector. . . . . . . . . . . . . . . . . 33 
3.5 The acceptance of the MINOS veto shield for cosmic ray muons . . . . 36

$3.6 \quad$ The stability of noise rate in the MINOS veto shield. . . . . . . . . . . 38

3.7 The gain of the PMTs that readout the MINOS veto shield. . . . . . . 38

3.8 The timing resolution of the MINOS veto shield. . . . . . . . . . . 40

$3.9 \quad$ Constants used to calibrate the veto shield timing. . . . . . . . . . . . 40

$4.1 \quad$ An overview of the MINOS DAQ architecture. . . . . . . . . . . . . 44

4.2 DAQ trigger efficiency for atmospheric $\nu_{\mu}$ CC events. . . . . . . . . . 46

4.3 The finite state machine for the DAQ run model. . . . . . . . . . . . . 50

5.1 The measured primary cosmic ray flux of protons and Helium nuclei. . 55

5.2 The weighted phase space distribution for atmospheric neutrinos. . . . 57

5.3 The atmospheric neutrino flux from the Battistoni et al calculations. . 59

5.4 Zenith angle distributions for the Battistoni et al atmospheric neutrino flux calculation. . . . . . . . . . . . . . . . 60

5.5 A schematic of the $\nu_{\mu}$ CC interaction. . . . . . . . . . 61

5.6 The contributions to the total $\nu_{\mu}$ charged current cross section from the different simulated processes in NEUGEN3. . . . . . . . . . . . . . 61

5.7 The total neutrino cross sections as a function of neutrino energy compared to data. . . . . . . . . . . . . . . . . . . . 62

5.8 The $\nu_{\mu}$ and $\bar{\nu}_{\mu}$ charged current cross section as a function of energy. . 63

5.9 Ratio of cross sections $\bar{\nu}_{\mu} / \nu_{\mu}$ as a function of energy as calculated by

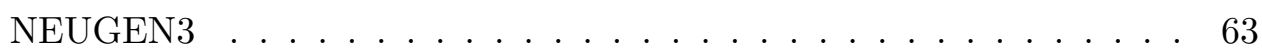

5.10 Data and Monte Carlo track direction reconstruction efficiency for cosmic ray muons that stop in the detector's fiducial volume. . . . . . . . 66

5.11 The timing resolution as a function of plane for an example run. . . . 66

5.12 The attenuation of charge associated with a hit as a function of muon

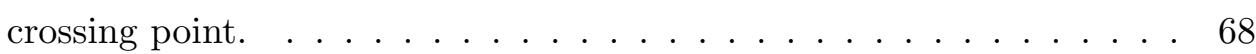

5.13 The probability of a hit on a cosmic ray muon track being double ended as a function of track position. . . . . . . . . . . . . 68

5.14 The charge deposited per unit strip length by a muon. . . . . . . . . . 69

5.15 The $\langle Q / P\rangle / \sigma_{Q / P}$ distribution for stopping cosmic ray muons for data and Monte Carlo. . . . . . . . . . . . . . . . . . 70

5.16 The dependance of $\left(N^{+} / N^{-}\right)_{\text {Data }} /\left(N^{+} / N^{-}\right)_{M C}$ on reconstructed muon momentum. . . . . . . . . . . . . . . . 70

$6.1 \quad$ The rate of through-going cosmic ray muons for both supermodules. . 73 
6.2 The reconstruction tracking efficiency for $\nu_{\mu}$ CC events. . . . . . . . 74

$6.3 \quad \nu_{\mu}$ charged current tracking purities. . . . . . . . . . . 76

6.4 The distance from event to the edge of the detector. . . . . . . . . . 77

6.5 The total charge deposited outside the fiducial volume. . . . . . . . . 78

6.6 The event trace as a function of energy. . . . . . . . . . . . . . 80

6.7 The trace for Monte Carlo signal and backgrounds. . . . . . . . . . 80

6.8 The zenith angle against vertex charge for cosmic muon background and signal . . . . . . . . . . . . . . . . . . . . . 81

6.9 The zenith angle against angle of track to planes over vertex charge for cosmic muon background and signal. . . . . . . . . . . . . . 81

6.10 The $\eta$ cut for Monte Carlo signal and background. . . . . . . . . . . . 82

6.11 An example of 'turn over' event. . . . . . . . . . . . . . . . . . . 83

6.12 The mean and RMS of the distribution of hits about the upper end of the track . . . . . . . . . . . . . . . . . . . 84

6.13 The true cosine of the angle to $X Y$-plane for the cosmic ray muon background. . . . . . . . . . . . . . . . . . . . 89

6.14 The veto shield efficiency from stopping cosmic ray muons as a function of $Z$ and time. . . . . . . . . . . . . . . . . . . . 90

6.15 The out-of-time charge in veto shield for selected events. . . . . . . . 92

6.16 The depth distribution of voted events. . . . . . . . . . . . . . . . 92

6.17 The signal efficiency. . . . . . . . . . . . . . . . . . . . . 96

6.18 Location of the selected events in the $X Y$-plane $\ldots \ldots \ldots$. . . . . . 97

6.19 The location of selected events in the detector volume. . . . . . . . . . 98

6.20 The time distribution of selected events. . . . . . . . . . . . . . . . 999

6.21 Some examples of selected events. . . . . . . . . . . . . 100

$7.1 \quad$ The difference in $\chi^{2} / n d f$ for the fits to the north and south going track hypotheses for $\nu_{\mu}$ CC tracks. . . . . . . . . . . . . . 104

7.2 The track direction reconstruction efficiency and purity. . . . . . . . . 104

7.3 Direction reconstruction efficiency for cosmic ray muons after a smearing factor was applied to the Monte Carlo $\chi^{2} / n d f$ distributions. . . . . 105

7.4 Neutrino zenith angle reconstruction resolution and bias. . . . . . . . 106

$7.5 \quad \cos \theta_{\text {zenith }}$ angle resolution from Monte Carlo. . . . . . . . . . . . . 107

$7.6 \quad$ Measured zenith angle distributions. . . . . . . . . . . . . . . . 108

7.7 Muon momentum resolution. . . . . . . . . . . . . . . . . . . . . . 109

7.8 Handornic shower energy resolution. . . . . . . . . . . . . . . . 110 
7.9 Measured neutrino energy distribution. . . . . . . . . . . . . . 111

7.10 Neutrino charge separation. . . . . . . . . . . . . . . . . . . . 114

7.11 Neutrino charge purity. . . . . . . . . . . . . . . . . 115

7.12 The $\langle Q / P\rangle / \sigma_{Q / P}$ distribution of selected events. . . . . . . . . . 115

7.13 Tuning the timing and charge identification cuts to optimise sensitivity to differences in $\Delta m^{2}$ and $\Delta \bar{m}^{2} \ldots \ldots \ldots \ldots 116$

7.14 Comparison of the expected sensitivity obtained from different fitting methods. . . . . . . . . . . . . . . . . . . . . . . . . . 119

7.15 The measured up-down ratio compared to Monte Carlo expectation as

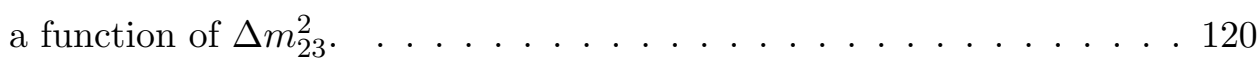

7.16 The $\log _{10}(L / E)$ resolution for events with good timing. . . . . . . . 121

7.17 The expected $\log _{10}(L / E)$ distribution for an exposure of 25 kton-years. 121

$7.18 \log _{10}(L / E)$ resolution as a function of neutrino energy and zenith angle. 122

7.19 The expected zenith angle/energy distributions for an exposure of 25 kton-years. . . . . . . . . . . . . . . . . . . . . . 124

7.20 Neutrino angle resolution, in degrees, as a function of neutrino energy and the fraction of the neutrino energy carried by the muon. . . . . . 125

7.21 The effect of neutrino flux normalisation uncertainty on the expected sensitivity. . . . . . . . . . . . . . . 126

7.22 The reconstructed energies of selected events using three different hadronic models. . . . . . . . . . . . . . . . . . . 127

7.23 zenith angle distributions for three different hadronic models. . . . . . 128

7.24 The effect of event timing simulation on the expected sensitivity. . . . 129

7.25 The effect of systematic uncertainty on the contribution of the quasielastic process on the total cross section on the expected sensitivity. . 130

7.26 The expected sensitivity from the fully contained events for a $25 \mathrm{kton}-$ year exposure. . . . . . . . . . . . . . . . . . . 132

7.27 The expected and observed zenith angle distribution for 2.52 ktonyears exposure. . . . . . . . . . . . . . . . . . . . . 134

7.28 The likelihood surface obtained for the selected events. . . . . . . . . . 135

7.29 The $68 \%$ and $90 \%$ excluded regions of oscillation parameter space. . . 136

7.30 The expected and observed charge separated zenith angle distributions. 138

7.31 The expected sensitivity to $\Delta m^{2}$ and $\Delta \bar{m}^{2} \ldots \ldots \ldots 139$

7.32 The expected confidence limits for the two extreme cases when $\Delta m_{23}^{2} \neq$

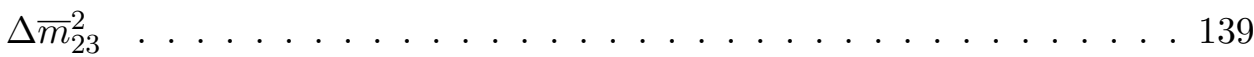




\section{List of Tables}

1.1 Summary of current limits on neutrino masses . . . . . . . . . . . . 2

$3.1 \quad$ Single and double ended noise rates in the veto shield. . . . . . . . . . 37

3.2 Timing resolution of the veto shield before and after calibration. . . . 39

4.1 The event and raw data rates for the Far Detector after the DAQ trigger. 45

4.2 The primary DAQ states. . . . . . . . . . . . . . . . . . . . . . . 49

6.1 The number of events (data and Monte Carlo) at difference states of the event selection. . . . . . . . . . . . . . . . . . . . 85

6.2 The veto shield efficiency for stopping cosmic ray muons. . . . . . . . 89

6.3 Summary of selected events compared to Monte Carlo expectations . . 101

7.1 Summary of the classifications of observed events into 'Low Resolution' and 'Good Timing' samples. . . . . . . . . . . . . . . . . . . . . 103

7.2 The charge of the observed events. . . . . . . . . . . . . 113 



\section{Chapter 1}

\section{Introduction}

In 1914 Chadwick observed that the energy spectrum from $\beta$-decay was continuous up to a maximum value [1]. If $\beta$-decay were a two body process, as it was then believed to be, a continuous spectrum would violate energy conservation. It was a problem that plagued the physics community for fifteen years, prompting DeBye to declare it "a problem like new taxes, one had best not think about it".

In 1930 Pauli proposed a solution in a letter to a conference in Türbingen. Pauli's solution was a new particle contained within the nucleus and ejected with the electron during $\beta$-decay. He called it the 'neutron' and deduced it must be light, neutral and spin $1 / 2$ to conserve energy, charge and angular momentum. It is interesting to note that Pauli's letter is cautious about his suggested solution, calling it "desperate".

A year later Chadwick discovered what we now know as the neutron for which he was awarded the Nobel prize in 1935 2. It was clearly not Pauli's particle as the mass was much too great. Fermi gave the neutrino its name in 1931 and in 1934 published his theory of nuclear $\beta$-decay [3]. The success of Fermi's theory gave the neutrino a solid theoretical grounding. However, the low probability of interaction made the direct observation of the neutrino exceptionally difficult and it was not until 20 years later that the neutrino was first observed. Fermi's neutrino was the 'electron neutrino', i.e. its creation and destruction is always associated with an electron. The discovery of heavier charged leptons, the muon $\left(\mu^{-}\right)$and tau $\left(\tau^{-}\right)$ suggested the existence of the muon and tau neutrinos. The muon neutrino was first observed in 1962 at Brookhaven National Laboratory [4] and the tau neutrino by the DONUT collaboration in 2000 [5].

Until recently the standard model of particle physics incorporates all three flavours of neutrinos and anti-neutrinos as massless particles. Efforts to directly measure the 
mass of the neutrinos have so far only yielded upper limits, shown in Table 1.1. In the standard model neutrinos interact purely through the weak force. There are two classes of neutrino interaction: neutral current,

$$
\nu_{x}+N \rightarrow \nu_{x}+N, x=e, \mu \text { or } \tau
$$

where the neutrino exchanges some momentum with the target particle $N$ but otherwise remains unchanged, and charged current,

$$
\begin{aligned}
& \nu_{x}+n \rightarrow x^{-}+p \\
& \bar{\nu}_{x}+p \rightarrow x^{+}+n
\end{aligned}
$$

where a charged lepton of the same flavour as the neutrino is produced.

\begin{tabular}{|l|c|}
\hline Flavour & Mass limit \\
\hline Electron & $<3 \mathrm{eV}$ \\
Muon & $<0.19 \mathrm{MeV}$ \\
Tau & $<18.2 \mathrm{MeV}$ \\
\hline
\end{tabular}

Table 1.1: The current limits on neutrino masses as summarised in [6]

In 1957, Pontecorvo suggested that if neutrinos have a non-zero mass, it may be possible for them to oscillate between flavours [7, 8]. The first hint that neutrino oscillation is a real phenomenon came in the 1960s when Ray Davis measured the flux of electron neutrinos from the sun and found it to be significantly lower than expected [9]. However, the first convincing evidence came from the SuperKamiokande collaboration's 1998 study of muon neutrinos produced in the Earth's atmosphere [10].

The MINOS-NuMI project is designed to accurately measure the frequency of oscillations using a man-made beam of neutrinos instead of natural sources, such as the atmosphere or the sun. The MINOS detector is the first deep underground neutrino detector to be magnetised and therefore able to determine the charge of the lepton produced in a neutrino interaction. While the detector was designed to detect neutrinos from the beam, it also presents the unique opportunity to independently measure the frequency of oscillations of atmospheric neutrinos and anti-neutrinos.

This thesis details the first attempt to measure atmospheric neutrino oscillation parameters using neutrinos interacting within the MINOS detector. The relatively small size of the data sample restricts the precision of the measurement obtained. 
In Chapter 2, the phenomenology of neutrino oscillations is introduced and the current experimental data is presented. Chapter 3 introduces the MINOS detector and discusses its construction and calibration. The design and implementation of the MINOS Data Acquisition system is discussed in Chapter 4. Chapter 5 discusses the source and simulation of atmospheric neutrinos in the MINOS detector. The task of isolating candidate atmospheric neutrino interactions from a large cosmic ray muon background is presented in Chapter 6. The extraction of the oscillation amplitude and frequency from the selected events and the future sensitivity of atmospheric neutrino oscillation measurements is presented in Chapter 7 . 


\section{Chapter 2}

\section{Neutrino Oscillations}

\subsection{Neutrino Oscillations In Vacuum}

Neutrinos are produced and detected in eigenstates of the weak interaction. However, there is no reason why the weak eigenstates should be parallel to the mass eigenstates. We can write the weak eigenstates $\left(\nu_{e}, \nu_{\mu}, \nu_{\tau}\right)$ as linear combinations of the mass eigenstates $\left(\nu_{1}, \nu_{2}, \nu_{3}\right)$,

$$
\left(\begin{array}{c}
\nu_{e} \\
\nu_{\mu} \\
\nu_{\tau}
\end{array}\right)=\left(\begin{array}{ccc}
U_{e 1} & U_{e 2} & U_{e 3} \\
U_{\mu 1} & U_{\mu 2} & U_{\mu 3} \\
U_{\tau 1} & U_{\tau 2} & U_{\tau 3}
\end{array}\right)\left(\begin{array}{c}
\nu_{1} \\
\nu_{2} \\
\nu_{3}
\end{array}\right)
$$

where $U$ is Maki-Nakagawa-Sakata (MNS) lepton mixing matrix [11].

Each mass eigenstate evolves as

$$
\left|\nu_{1}(\mathbf{x})\right\rangle=\left|\nu_{1}(0)\right\rangle e^{-i \mathbf{p}_{1} \cdot \mathbf{x}}
$$

where $\mathbf{p}_{\mathbf{1}}$ is the 4 -momentum of the state $\mathbf{1}$. Therefore, for a neutrino produced in an initially pure $\nu_{\mu}$ state, the wavefunction evolves as:

$$
\left|\nu_{\mu}(\mathbf{x})\right\rangle=U_{\mu 1}\left|\nu_{1}\right\rangle e^{-i \mathbf{p}_{1} \cdot \mathbf{x}}+U_{\mu 2}\left|\nu_{2}\right\rangle e^{-i \mathbf{p}_{\mathbf{2}} \cdot \mathbf{x}}+U_{\mu 3}\left|\nu_{3}\right\rangle e^{-i \mathbf{p}_{\mathbf{3}} \cdot \mathbf{x}} .
$$

By inverting Equation 2.1 to express the mass eigenstates in terms of weak eigen- 
states and substituting in for $\left|\nu_{1}\right\rangle,\left|\nu_{2}\right\rangle$ and $\left|\nu_{3}\right\rangle$, we obtain;

$$
\begin{aligned}
\left|\nu_{\mu}(\mathbf{x})\right\rangle & =\left(U_{\mu 1} U_{e 1}^{*} e^{-i \mathbf{p}_{1} \cdot \mathbf{x}}+U_{\mu 2} U_{e 2}^{*} e^{-i \mathbf{p}_{2} \cdot \mathbf{x}}+U_{\mu 3} U_{e 3}^{*} e^{-i \mathbf{p}_{3} \cdot \mathbf{x}}\right)\left|\nu_{e}\right\rangle \\
& +\left(U_{\mu 1} U_{\mu 1}^{*} e^{-i \mathbf{p}_{1} \cdot \mathbf{x}}+U_{\mu 2} U_{\mu 2}^{*} e^{-i \mathbf{p}_{2} \cdot \mathbf{x}}+U_{\mu 3} U_{\mu 3}^{*} e^{-i \mathbf{p}_{3} \cdot \mathbf{x}}\right)\left|\nu_{\mu}\right\rangle \\
& +\left(U_{\mu 1} U_{\tau 1}^{*} e^{-i \mathbf{p}_{1} \cdot \mathbf{x}}+U_{\mu 2} U_{\tau 2}^{*} e^{-i \mathbf{p}_{2} \cdot \mathbf{x}}+U_{\mu 3} U_{\tau 3}^{*} e^{-i \mathbf{p}_{3} \cdot \mathbf{x}}\right)\left|\nu_{\tau}\right\rangle
\end{aligned}
$$

Therefore, for a non-diagonal form of $U$, the wavefunction for $\nu_{\mu}$ has a non-zero contribution from $\nu_{e}$ and $\nu_{\tau}$. The probability of observing a $\nu_{\mu}$ at $\mathbf{x}$, can be obtained by projecting out the $\nu_{\mu}$ component. By rearranging and using the unitary relations for $U_{i j}$ we obtain;

$$
\begin{aligned}
P\left(\nu_{\mu} \rightarrow \nu_{\mu}\right)=\left|\left\langle\nu_{\mu} \mid \nu_{\mu}(\mathbf{x})\right\rangle\right|^{2}=1 & -4\left|U_{\mu 1}\right|^{2}\left|U_{\mu 2}\right|^{2} \sin ^{2}\left(\frac{\left(\mathbf{p}_{\mathbf{1}}-\mathbf{p}_{\mathbf{2}}\right) \cdot \mathbf{x}}{2}\right) \\
& -4\left|U_{\mu 1}\right|^{2}\left|U_{\mu 3}\right|^{2} \sin ^{2}\left(\frac{\left(\mathbf{p}_{\mathbf{1}}-\mathbf{p}_{\mathbf{3}}\right) \cdot \mathbf{x}}{2}\right) \\
& -4\left|U_{\mu 2}\right|^{2}\left|U_{\mu 3}\right|^{2} \sin ^{2}\left(\frac{\left(\mathbf{p}_{\mathbf{3}}-\mathbf{p}_{\mathbf{2}}\right) \cdot \mathbf{x}}{2}\right) .
\end{aligned}
$$

Making the assumption that the energy of the neutrino is much greater than the mass, $E \gg m$, and that the neutrino is produced with a well defined energy, so that $\Delta \mathbf{p} \cdot \mathbf{x} \approx(\Delta E-\Delta p) x$ and $p \approx E-m^{2} / 2 E$, where $\underline{x}$ is the 3 -vector position, $x=|\underline{x}|$ and $\Delta \mathbf{p}$ is the difference in momentum between mass states. Therefore we may write:

$$
\begin{aligned}
P\left(\nu_{\mu} \rightarrow \nu_{\mu}\right)=1 & -4\left|U_{\mu 1}\right|^{2}\left|U_{\mu 2}\right|^{2} \sin ^{2}\left(\frac{\Delta m_{12}^{2} L}{4 E}\right) \\
& -4\left|U_{\mu 1}\right|^{2}\left|U_{\mu 3}\right|^{2} \sin ^{2}\left(\frac{\Delta m_{13}^{2} L}{4 E}\right) \\
& -4\left|U_{\mu 2}\right|^{2}\left|U_{\mu 3}\right|^{2} \sin ^{2}\left(\frac{\Delta m_{32}^{2} L}{4 E}\right),
\end{aligned}
$$

where $L=|\underline{x}|$, the distance travelled by the neutrino, and $\Delta m_{i j}^{2}=m_{i}^{2}-m_{j}^{2}$. Similar equations can be derived for all neutrino transition probabilities. In general, the transition probability between two weak eigenstates $\alpha$ and $\beta$ can be written as

$$
P\left(\nu_{\alpha} \rightarrow \nu_{\beta}\right)=\delta_{\alpha \beta}-2 \Re \sum_{j>i} U_{\alpha i} U_{\alpha j}^{*} U_{\beta i}^{*} U_{\beta j}\left(1-e^{\left(i \Delta m_{i j}^{2} L / 2 E\right)}\right),
$$

where $j$ and $i$ represent the mass eigenstates.

In the case of normal hierarchy $\left(m_{1}<m_{2}<m_{3}\right)$ the mass difference can be 
related by $\Delta m_{12}^{2}+\Delta m_{23}^{2}=\Delta m_{13}^{2}$. The full hierarchy of the masses $m_{1}, m_{2}$ and $m_{3}$ is not known, as the sign of the $\Delta m^{2}$ cannot be determined from vacuum oscillation experiments. The two well-established measurements of neutrino oscillations give two very different $\Delta m^{2}$ s. The solar neutrino experiments (discussed in Section 2.3) are consistent with a $\left|\Delta m^{2}\right| \approx 8 \times 10^{-5} \mathrm{eV}^{2}$ and atmospheric neutrino experiments (discussed in Section 2.5) are consistent with a $\left|\Delta m^{2}\right| \approx 2 \times 10^{-3} \mathrm{eV}^{2}$. We can arbitrarily choose our basis such that the separation between $m_{1}$ and $m_{2}$ relates to the solar neutrino measurement. Therefore,

$$
\left|\Delta m_{12}^{2}\right| \ll\left|\Delta m_{13}^{2}\right| \approx\left|\Delta m_{23}^{2}\right|
$$

which is independent of mass hierarchy. The MNS matrix can be factorised into a convenient form [12] based on the three mixing angles between mass eigenstates $\theta_{i j}$ and a complex phase factor $\delta$, so that

$$
U=\left(\begin{array}{ccc}
1 & 0 & 0 \\
0 & c_{23} & s_{23} \\
0 & -s_{23} & c_{23}
\end{array}\right)\left(\begin{array}{ccc}
c_{13} & 0 & s_{13} e^{-i \delta} \\
0 & 1 & 0 \\
-s_{13} e^{i \delta} & 0 & c_{13}
\end{array}\right)\left(\begin{array}{ccc}
c_{12} & s_{12} & 0 \\
-s_{12} & c_{12} & 0 \\
0 & 0 & 1
\end{array}\right)
$$

where $c_{i j}=\cos \theta_{i j}$ and $s_{i j}=\sin \theta_{i j}$ and Dirac neutrino masses are assumed. The Chooz result (discussed in Section 2.2) suggests that $\theta_{13}$ is small and therefore that the second term is approximately the identity matrix. In this case the atmospheric sector (the first term) is de-coupled from the solar sector (the third term). Therefore, instead of the general form of the oscillation probability, given in Equation 2.7, we can analyse many of the current oscillation results in terms of two generation mixing. In this case, the form of $U$ reduces to

$$
\left(\begin{array}{l}
\nu_{\alpha} \\
\nu_{\beta}
\end{array}\right)=\left(\begin{array}{cc}
\cos \theta & \sin \theta \\
-\sin \theta & \cos \theta
\end{array}\right)\left(\begin{array}{l}
\nu_{1} \\
\nu_{2}
\end{array}\right)
$$

where $\theta$ is the mixing angle between the generations. By following the same procedure as above, we arrive at a simpler form for the oscillation probability:

$$
P\left(\nu_{\alpha} \rightarrow \nu_{\beta}\right)=\delta_{\alpha \beta}-\sin ^{2} 2 \theta \sin ^{2}\left(\Delta m^{2} L / 4 E\right)
$$

Using this simple form, we can study the effect of the mixing parameters on the oscillation probability. The mixing angle between generations, $\theta$, governs the size of the oscillations and the period of oscillation is governed by the mass difference $\Delta m^{2}$. 


\subsection{The Chooz Experiment}

The Chooz experiment [13] was a short baseline reactor experiment located approximately $1 \mathrm{~km}$ from the Chooz nuclear power plant on the France-Belgium border. The detector was a 5-ton Gadolinium (Gd) loaded liquid scintillator detector. $\bar{\nu}_{e}$ produced in the reactor were detected via the inverse $\beta$-decay reaction

$$
\bar{\nu}_{e}+p \rightarrow e^{+}+n
$$

The event signature is a delayed coincidence between the prompt back-to-back $\gamma$ signal from the $e^{+}$annihilation and the delayed signal from the neutron capture on the Gd atoms.

The measured $\bar{\nu}_{e}$ flux was compared to the Monte Carlo expectation and no evidence of neutrino oscillations was found. The mean energy of the $\bar{\nu}_{e}$ is $3 \mathrm{MeV}$. For neutrinos of this energy the oscillation length due to $\Delta m_{12}^{2}$, as measured by solar neutrino experiments, is of the order of $10 \mathrm{~km}$, large compared to the baseline of the experiment. Therefore, from Equation 2.7 the survival probability of the $\bar{\nu}_{e}$ can be written as,

$$
\begin{aligned}
P\left(\bar{\nu}_{e} \rightarrow \bar{\nu}_{e}\right) & \approx 1-4\left(1-U_{e 3}^{2}\right) U_{e 3}^{2} \sin ^{2}\left(\Delta m_{23}^{2} L / 4 E\right) \\
& =1-4\left(1-s_{13}^{2}\right) s_{13}^{2} \sin ^{2}\left(\Delta m_{23}^{2} L / 4 E\right) \\
& =1-\sin ^{2} 2 \theta_{13} \sin ^{2}\left(\Delta m_{23}^{2} L / 4 E\right) .
\end{aligned}
$$

Figure 2.1 shows the resulting exclusion plot in terms of $\Delta m_{23}^{2}$ and $\sin ^{2} 2 \theta_{13}$ obtained. From atmospheric neutrino oscillation results $\Delta m_{23}^{2}$ is known to be in the range $10^{-3}$ to $10^{-2}$. This places a limit on $\sin ^{2} 2 \theta_{13} \geq 0.17$ at $90 \%$ confidence. Therefore there are two possible allowed regions of $s_{13}, s_{13} \geq 0.96$ or $s_{13} \leq 0.04$. The ambiguity in $s_{13}$ is removed by the atmospheric and solar neutrino results. The Palo Verde experiment also did not observe an oscillation signature, however it placed weaker limits on the mixing paramters than Chooz [14].

\subsection{The Solar Neutrino Problem}

The thermonuclear fusion process in the sun's core produces a large flux of electron neutrinos. The dominant source of solar energy is the fusion process

$$
4 p \rightarrow{ }^{4} \mathrm{He}+2 \nu_{e}+2 e^{+},
$$




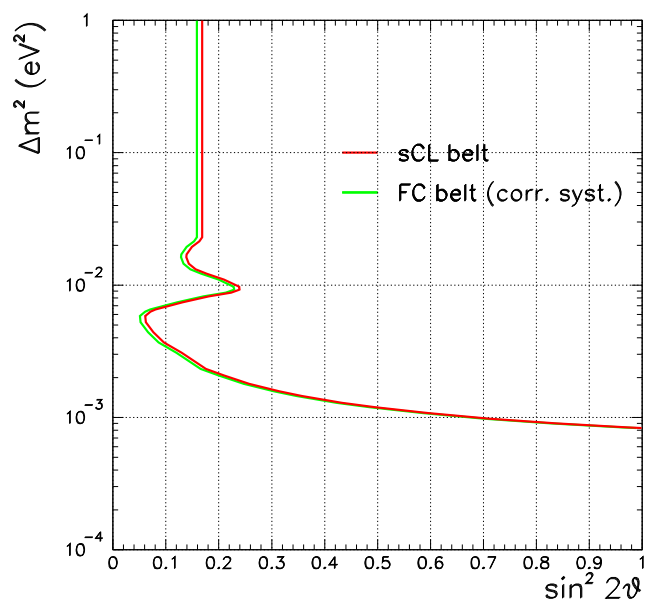

Figure 2.1: The confidence limits on $\sin ^{2} 2 \theta_{13}$ and $\Delta m_{23}^{2}$ obtained by the Chooz experiment. All points to the right of the lines are excluded at a confidence of $90 \%$. The two lines are the result of two different methods of obtaining the confidence limits. Figure taken from [13].

which proceeds via a series of reactions known as the proton-proton chain.

The expected energy spectrum of electron neutrinos calculated using the Standard Solar Model (SSM) [15], is shown in Figure 2.2. The sun's luminosity is highly correlated with the neutrino flux, therefore the observed luminosity of the sun places strict limits the SSM. The estimated uncertainty in the flux of electron neutrinos is of the order of a few percent. However, the neutrino flux has been measured by several experiments and has shown a significant deficit compared to predictions in the absence of neutrino oscillations.

The solar neutrino experiments can be broadly divided into two categories; the gallium and chlorine radiochemical detectors and the water Cerenkov experiments. The energy range accessible to these experiments is shown in Figure 2.2. Before the experiments are introduced, the phenomenology of solar neutrino oscillations is briefly discussed below.

Neutrinos from the sun have an energy of the order of $1 \mathrm{MeV}$, so the oscillation length corresponding to $\Delta m_{23}^{2}$ is of order $1 \mathrm{~km}$ and is small compared to the region of production within the sun's core. The component of oscillation probability due to $\Delta m_{23}^{2}$ is therefore averaged to $1 / 2$, and the general form of the vacuum oscillation 


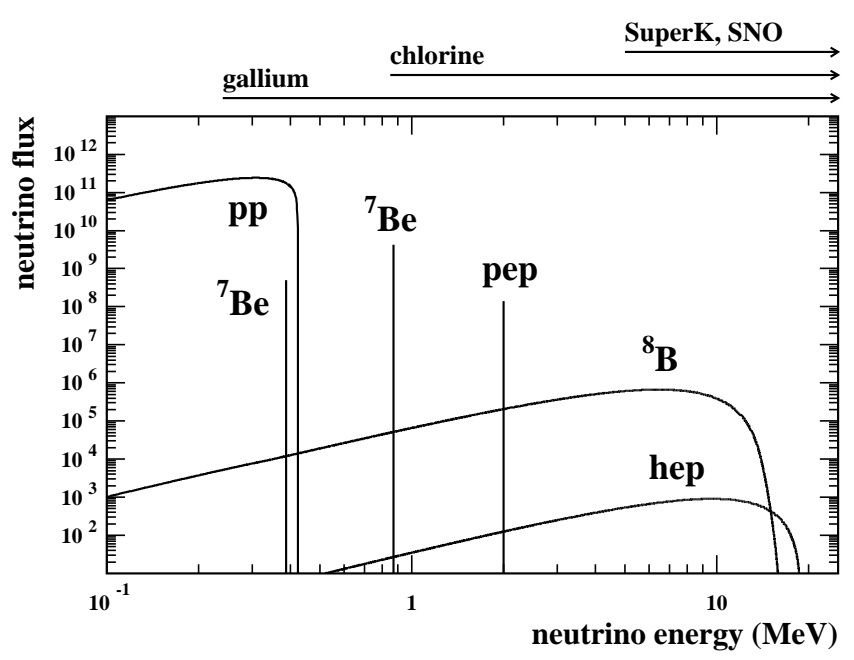

Figure 2.2: The solar neutrino flux at the Earth from the standard solar model [15]. The flux is measured in units of $\mathrm{cm}^{-1} \mathrm{~s}^{-1} \mathrm{MeV}^{-1}$ for the continuous spectra and $\mathrm{cm}^{-1} \mathrm{~s}^{-1}$ for the lines. The regions over which the different class of experiments are sensitive are also shown, Figure taken from [16].

probability, Equation 2.7, can be written for solar neutrinos as

$$
\begin{aligned}
P\left(\nu_{e} \rightarrow \nu_{e}\right) & \approx 1-4 U_{e 1}^{2} U_{e 2}^{2} \sin ^{2}\left(\Delta m_{12}^{2} L / 4 E\right)-2\left(1-U_{e 3}^{2}\right) U_{e 3}^{2} \\
& =s_{13}^{4}+c_{13}^{4}\left(1-4 c_{12}^{4} s_{12}^{4} \sin ^{2}\left(\Delta m_{12}^{2} L / 4 E\right)\right) \\
& =s_{13}^{4}+c_{13}^{4}\left(1-\sin ^{2} 2 \theta_{\odot} \sin ^{2}\left(\Delta m_{\odot}^{2} L / 4 E\right)\right),
\end{aligned}
$$

where $\sin ^{2} 2 \theta_{\odot}=4 c_{12}^{2} s_{12}^{2}$ and $\Delta m_{\odot}^{2}=\Delta m_{12}^{2}$. However, the oscillation probability is strongly affected by the material through which the neutrino passes. All neutrinos elastically scatter from electrons and nuclei in the medium through which they propagate. However, for electron neutrinos there is an additional component from charged current forward scattering on electrons. Therefore, electron neutrinos experience an additional effective potential in the medium, altering their phase with respect to the other flavours. The introduction of matter effects breaks the independence of the oscillation probability on the sign of $\Delta m_{12}^{2}$ and gives effective values of $\sin ^{2} 2 \theta_{\odot}$ and $\Delta m_{\odot}^{2}$. Solar neutrino experiments can therefore, in principle, determine the mass hierarchy between the $\nu_{1}$ and $\nu_{2}$ mass states.

As discussed in the previous section, the null result from Chooz gives two allowed 
regions for $U_{e 3}$. However, a large mixing angle $\theta_{13}$ would give a solar electron neutrino survival probability close to unity regardless of the sizes of $\sin ^{2} \theta_{\odot}$ and $\Delta m_{\odot}^{2}$. Hence, only a small mixing angle, $\theta_{13}$ is consistent with the observed solar neutrino deficit and Equation 2.13 reduces to the simple two flavour case.

\subsubsection{Radiochemical Experiments}

The first indication of the solar neutrino deficit came from the Homestake experiment [9] which consisted of a $380 \mathrm{~m}^{3}$ tank filled with tetrachlorethylene, $\mathrm{C}_{2} \mathrm{Cl}_{4}$. The Homestake experiment detected neutrinos via the inverse $\beta$-decay process

$$
\nu_{e}+{ }^{37} \mathrm{Cl} \rightarrow e^{-}+{ }^{37} \mathrm{Ar},
$$

with a threshold energy of $0.814 \mathrm{MeV}$. The tank was purged with helium once a month to remove the argon atoms which were then counted by detecting the decay back to chlorine with a half-life of 35 days. The experiment released its first results in 1968 but continued to take data until 1995. The measured flux [17] was $2.56 \pm 0.16 \pm 0.16 \mathrm{SNU}$ compared to a predicted flux of $7.6_{-1.1}^{+1.3}$ SNU. One SNU (Solar Neutrino Unit) equals one neutrino interaction per second per $10^{36}$ atoms.

The gallium experiments SAGE and GALLEX/GNO are sensitive to the lower energy neutrinos from the sun, as shown in Figure 2.2, in particular those produced by the $p p$ chain. The $p p$ source is more directly correlated with the sun's luminosity than the higher energy sources $\left({ }^{7} B e\right.$ and $\left.{ }^{8} B\right)$ and the uncertainty in the neutrino fluxes is correspondingly lower.

The gallium experiments detect neutrinos via the reaction

$$
\nu_{e}+{ }^{71} \mathrm{Ga} \rightarrow e^{-}+{ }^{71} \mathrm{Ge},
$$

where the energy threshold is $0.233 \mathrm{MeV}$. The ${ }^{71} \mathrm{Ge}$ atoms are then purged from the system and counted by counting decays with a half-life of 16.5 days. The SAGE experiment measured a overall flux of $70.9_{-5.2}^{+5.3+3.7} \mathrm{SNU}$ [18] and GALLEX/GNO $70.8 \pm 4.5 \pm 3.8 \mathrm{SNU}$ [19] which are both incompatible with the SSM prediction of $129_{-7}^{+9} \mathrm{SNU}$. 


\subsubsection{Water Čerenkov Detectors}

\section{Kamiokande and Super-Kamiokande}

The Super-Kamiokande experiment consists of a large $40 \mathrm{~m}$ high and $40 \mathrm{~m}$ diameter cylinder filled with 50 ktons of ultra-pure water. The volume is divided into an outer an inner detector. The inner detector is $32 \mathrm{ktons}$, of which $22.5 \mathrm{ktons}$ is used as a fiducial volume and is viewed by 11146 photomultiplier tubes. The outer detector is used as a veto shield to identify through-going cosmic ray muons and low energy external backgrounds. Neutrinos are detected via the elastic scattering process

$$
\nu_{x}+e^{-} \rightarrow \nu_{x}+e^{-},
$$

where $x=e, \mu$, or $\tau$. The cross section for electron neutrinos is approximately five times greater than for than muon or tau neutrinos due to an additional contribution from charged current scattering. The recoil electron can be detected by the Čerenkov light it produces.

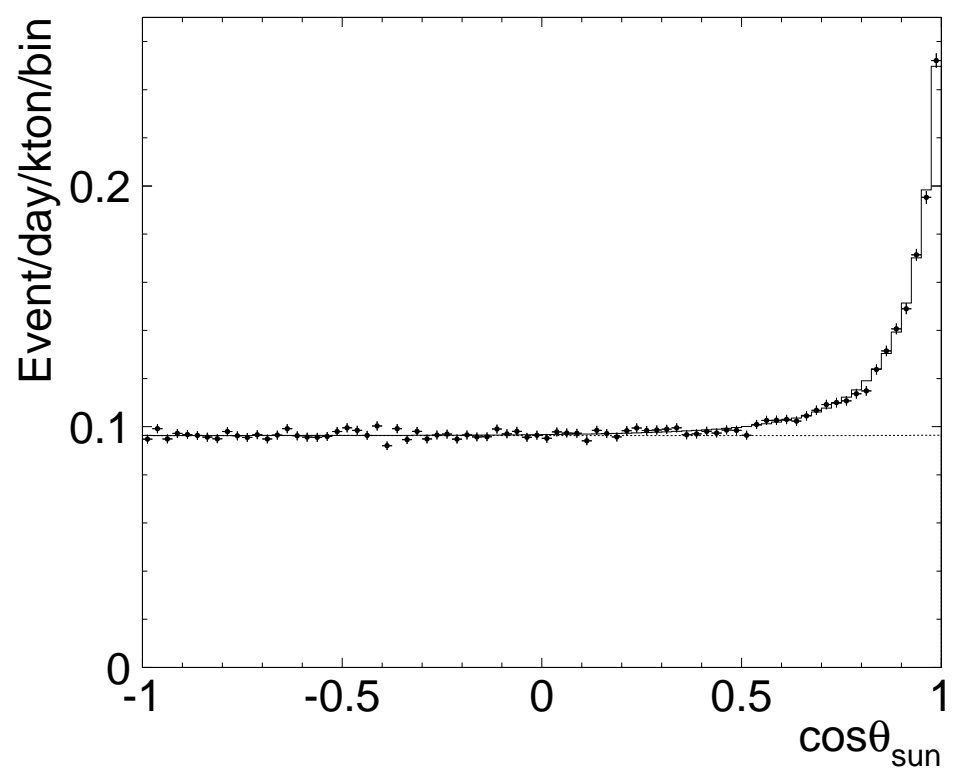

Figure 2.3: The cosine of the angle between the reconstructed electron direction and the Earth-Sun distance in the Super-Kamiokande detector for 1258 days of data. The points represent the data and the line is the prediction of backgrounds plus the best fit solar neutrino flux. Figure taken from [20]. 
The Super-Kamiokande experiment has two advantages over the radiochemical experiments, as both the direction of the neutrino and a lower limit on its energy can be estimated from the direction and energy of the recoil electron. Solar neutrinos can therefore be selected from the background by requiring events that point back towards the sun. Figure 2.3 shows the angle between the event direction and the sun. Super-Kamiokande measured the flux from elastic scattering processes to be $2.32 \pm 0.03_{-0.07}^{+0.08} \times 10^{6} \mathrm{~cm}^{-2} \mathrm{~s}^{-1}[20$ ], or $0.45 \pm 0.08$ of the expected SSM value. In addition to the integrated flux measurement, Super-Kamiokande was also able to look for distortions of the energy spectrum of the observed events and neutrino path-length $(L)$ effects, the so called "zenith spectrum" measurements. However, no significant distortions were observed excluding large regions of oscillation parameter space. Figure 2.4 shows the region of parameter space excluded by the zenith angle measurements. By combining the integrated flux measurements and the energy and path-length distributions, Super-Kamiokande obtained the two allowed regions shown in Figure 2.4 the Large Mixing Angle (LMA) solution at $\Delta m^{2}=10^{-4}$ and the LOW solution at $\Delta m^{2}=10^{-8}$.

\section{Sudbury Neutrino Observatory}

The first direct observation of solar neutrinos changing flavour was presented by the Sudbury Neutrino Observatory (SNO) experiment in 2001 [21]. SNO is a water Čherenkov detector located $2 \mathrm{~km}$ underground at the Creighton mine, Sudbury, Canada. The detector consists of 1 ktons of ultra-pure heavy water $\left(\mathrm{D}_{2} \mathrm{O}\right)$ surveyed by 9,456 photomultiplier tubes. The heavy water is surrounded by a further $7 \mathrm{ktons}$ of ultra-pure light water which acts as support and shielding. SNO detects solar ${ }^{8} B$ neutrinos via three different processes:

1. Elastic Scattering (ES). The scattering of all neutrino flavours from electrons,

$$
\nu_{x}+e^{-} \rightarrow \nu_{x}+e^{-},
$$

where $\nu_{x}=e, \mu$ or $\tau$. As discussed above, the cross section for electron neutrino is substantially higher for electron neutrinos than for the other flavours and is, therefore, most sensitive to the flux of electron neutrinos.

2. Charged-current (CC) interactions. Specific to electron neutrinos,

$$
\nu_{e}+d \rightarrow p+p+e^{-}
$$




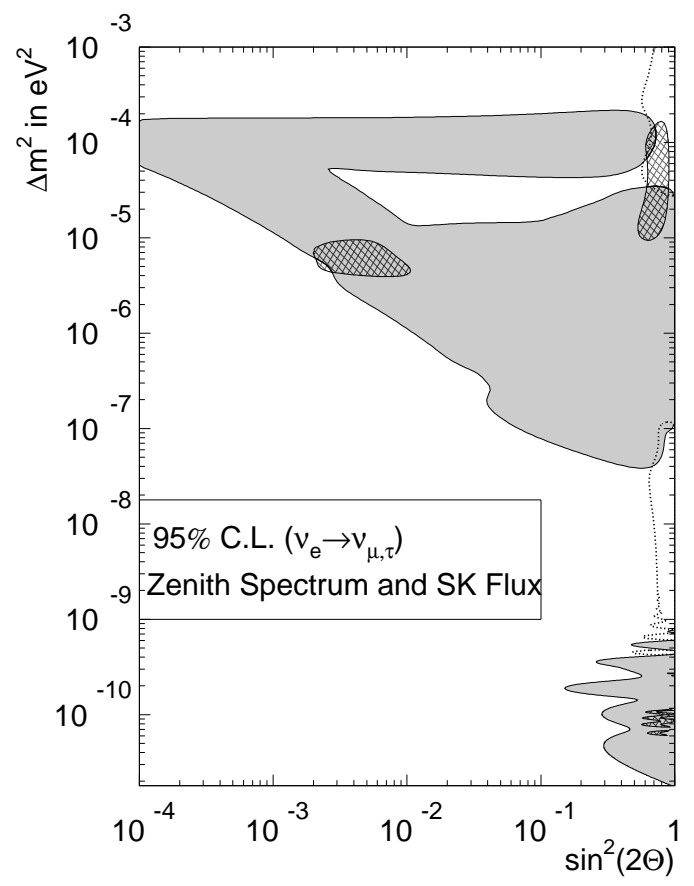

Figure 2.4: The allowed regions of vacuum oscillation parameter space from the integrated flux measurements is shown by the hatched areas. The region excluded from the absence of a distortion in the energy spectrum or path length distribution is shown by the shaded area. The allowed regions from a combination of SuperKamiokande's flux, energy spectrum and path length distribution is shown by the dotted line. Figure taken from [20].

The charged current interactions provides a direct measurement of the electron neutrino flux.

3. Neutral-current (NC) interactions. Equally sensitive to all neutrino flavours.

$$
\nu_{x}+d \rightarrow n+p+\nu_{x} .
$$

The neutron is detected using different methods during the three phases of the experiment.

SNO is unique as it is the only experiment that can simultaneously observe the disappearance of electron neutrinos and the corresponding appearance of other neutrino species via the NC interaction. The electrons are identified by the Cerenkov light they produce. During the first phase of the experiment the neutrons from NC 
interactions are detected via their capture on deuterium nuclei and subsequent emission of a $6.25 \mathrm{MeV}$ gamma-ray. In the second phase, salt $(\mathrm{NaCl})$ was added to the heavy water increasing the neutron capture efficiency and a gamma energy release which is better separated from background. During the third phase, due to start in 2005, the salt will be removed and an array of proportional counters will be inserted, which will directly detect the neutrons. The measured flux [22] for the three processes, ES, CC and NC are shown in Figure 2.5.

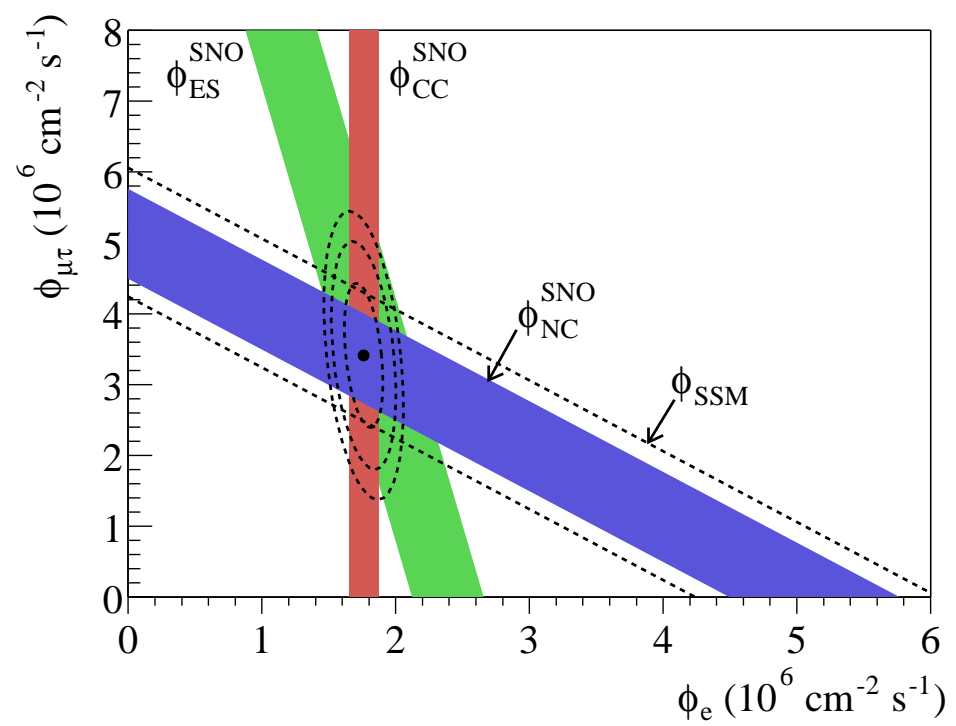

Figure 2.5: The ${ }^{8} \mathrm{~B}$ solar neutrino flux as measured by the SNO experiment. The Standard Solar Model (SSM) prediction is shown by the dotted line. The total flux measured with the neutral current reaction is shown by the solid diagonal band. The width of the bands represent the $\pm 1 \sigma$ errors. The results are consistent with neutrino flavour transformation at the $5.3 \sigma$ level. Figure taken from [22].

The combined fit to all the solar neutrino data with the results of the SNO experiment can be seen in left panel of Figure 2.6(a).

\subsection{KamLAND}

The Kamioka Liquid scintillator Anti-Neutrino Detector (KamLAND) was designed to confirm the observations of solar neutrino oscillation experiments. The experiment detects $\bar{\nu}_{e}$ from 53 Japanese power stations located at distances between 150 and 


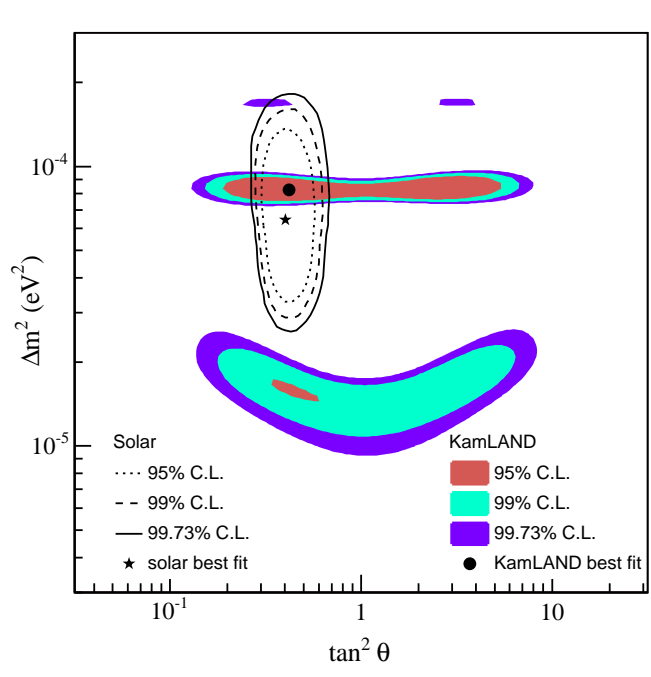

(a)

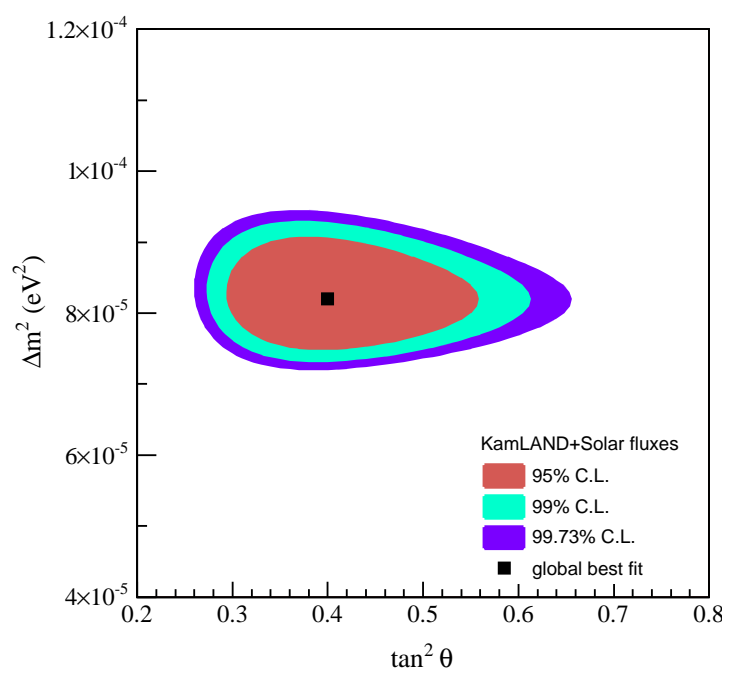

(b)

Figure 2.6: (a) The allowed regions obtained by the KamLAND experiment (solid regions) and the results of the solar neutrino experiments (lines). (b) The result of combining the KamLAND and solar neutrino results. Figure taken from [23].

$210 \mathrm{~km}$ from the detector. The survival probability for $\bar{\nu}_{e}$ is given by

$$
\begin{aligned}
P\left(\bar{\nu}_{e} \rightarrow \bar{\nu}_{e}\right) & \approx 1-4 U_{e 1}^{2} U_{e 2}^{2} \sin ^{2}\left(\Delta m_{12}^{2} L / 4 E\right)-4\left(1-U_{e 3}^{2}\right) U_{e 3}^{2} \sin ^{2}\left(\Delta m_{32}^{2} L / 4 E\right) \\
& =1-\cos ^{4} \theta_{13} \sin ^{2} 2 \theta_{12} \sin ^{2}\left(\Delta m_{12}^{2} L / 4 E\right)-\sin ^{2} 2 \theta_{13} \sin ^{2}\left(\Delta m_{32}^{2} L / 4 E\right) .
\end{aligned}
$$

As the mixing angle $\theta_{13}$ is small, KamLAND is primarily sensitive to the same oscillation parameters as the solar neutrino experiments.

The KamLAND detector consists of 1 kton of ultra-pure liquid scintillator contained in a transparent balloon suspended in non-scintillating mineral oil. The whole volume is surrounded by an $18 \mathrm{~m}$ support structure on which are mounted 1879 photomultiplier tubes. A 3.2 kton water Čerenkov detector surrounds the inner detector and is used to absorb neutrons and $\gamma$-rays from the surrounding rock and to tag through-going cosmic ray muons.

Electron anti-neutrinos are detected by the charged current interaction

$$
\bar{\nu}_{e}+p \rightarrow e^{+}+n,
$$


with a threshold of $1.8 \mathrm{MeV}$. The signal is scintillation light from the $e^{+}$and a delayed $2.2 \mathrm{MeV} \gamma$-ray from the neutron capture on hydrogen. The energy of the $\bar{\nu}_{e}$ is estimated from the $e^{+}$scintillation light.

The latest results from KamLAND 23 . show a distortion in the energy spectrum, shown in Figure 2.7. The result of a fit to the oscillation parameters for KamLAND data is shown in Figure 2.6(a), Also shown in Figure 2.6(a) are the confidence limits obtained from a fit to all the solar data. If it assumed that the neutrino and antineutrino mixing parameters are the same, the KamLAND result can be combined with the solar neutrino results to obtain a global fit, the allowed regions are shown in Figure 2.6(b), The best fit point is $\Delta m_{12}^{2}=8.2_{-0.5}^{+0.6} \mathrm{eV}^{2}$ and $\tan ^{2} \theta_{12}=0.40_{-0.07}^{+0.09}[23$.

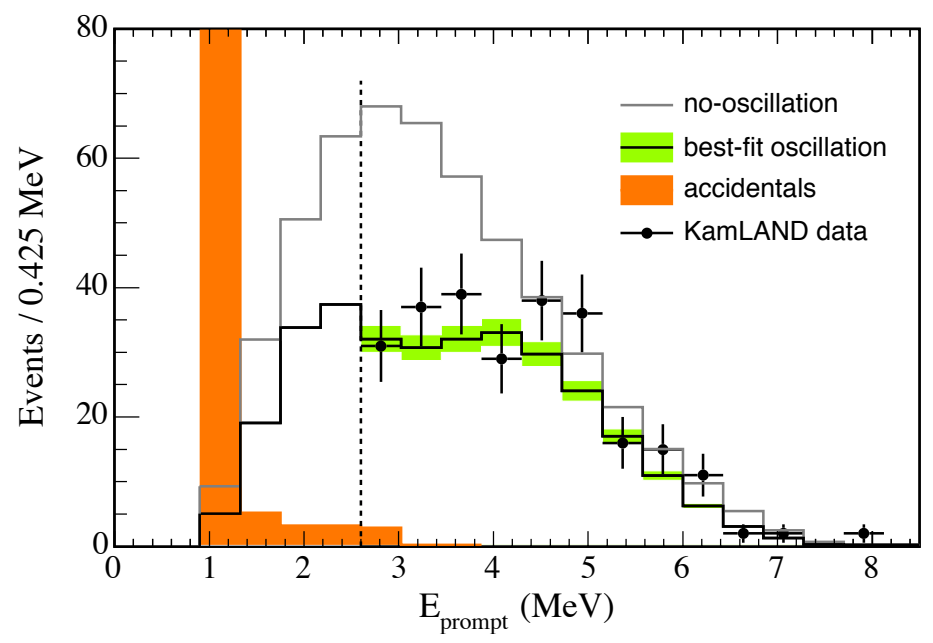

Figure 2.7: The measured energy spectrum of the $\bar{\nu}_{e}$ events measured by the KamLAND experiment. Figure taken from [23]. 


\subsection{Atmospheric Neutrinos}

Atmospheric neutrinos are produced in the decay of pions and kaons created by primary cosmic ray interactions with the Earth's atmosphere. The processes can be summarised as;

$$
\begin{aligned}
p+N & \rightarrow \pi^{ \pm}+X \\
\pi^{ \pm} & \rightarrow \mu^{ \pm}+\nu_{\mu}\left(\bar{\nu}_{\mu}\right) \\
\mu^{ \pm} & \rightarrow e^{ \pm}+\nu_{e}\left(\bar{\nu}_{e}\right)+\nu_{\mu}\left(\bar{\nu}_{\mu}\right) .
\end{aligned}
$$

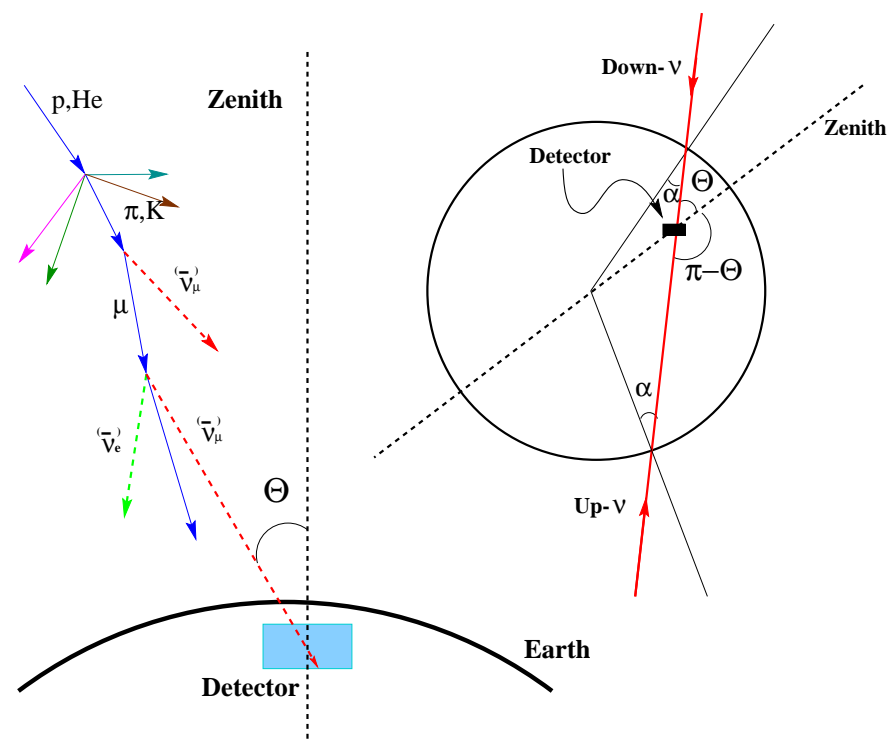

Figure 2.8: A sketch of the production of atmospheric neutrinos and the dependance of the neutrino path length on the zenith angle, $(\Theta)$.

The energy of atmospheric neutrinos contained within detectors is approximately $1 \mathrm{GeV}$. The path length for an atmospheric neutrino is the distance from its production point to the detector and varies from a few kilometres for neutrinos produced directly above the detector, to approximately $12,000 \mathrm{~km}$ for neutrinos created on the other side of the Earth, see Figure 2.8. The wavelength of oscillations due to the $\Delta m_{12}^{2}$ term, measured by solar neutrino experiments, is of the order of $40,000 \mathrm{~km}$ and therefore large compared with typical atmospheric neutrino path lengths. Therefore, oscillations containing $\Delta m_{12}^{2}$ do not have significant time to develop and we can 
write the probability of $\nu_{\mu} \rightarrow \nu_{\tau}$ oscillations as

$$
\begin{aligned}
P\left(\nu_{\mu} \rightarrow \nu_{\tau}\right) & \approx 4 U_{\mu 3}^{2} U_{\tau 3}^{2} \sin ^{2}\left(\Delta m_{23}^{2} L / 4 E\right) \\
& =\left(1-s_{13}^{2}\right) \sin ^{2} 2 \theta_{23} \sin ^{2}\left(\Delta m_{23}^{2} L / 4 E\right) .
\end{aligned}
$$

Again we assume $\theta_{13}$ is small, so that the transition probability reduces to the two flavour case. A diagram showing the oscillation probability as a function of neutrino zenith angle (defined in Figure 2.8) and energy is given in Figure 2.9.
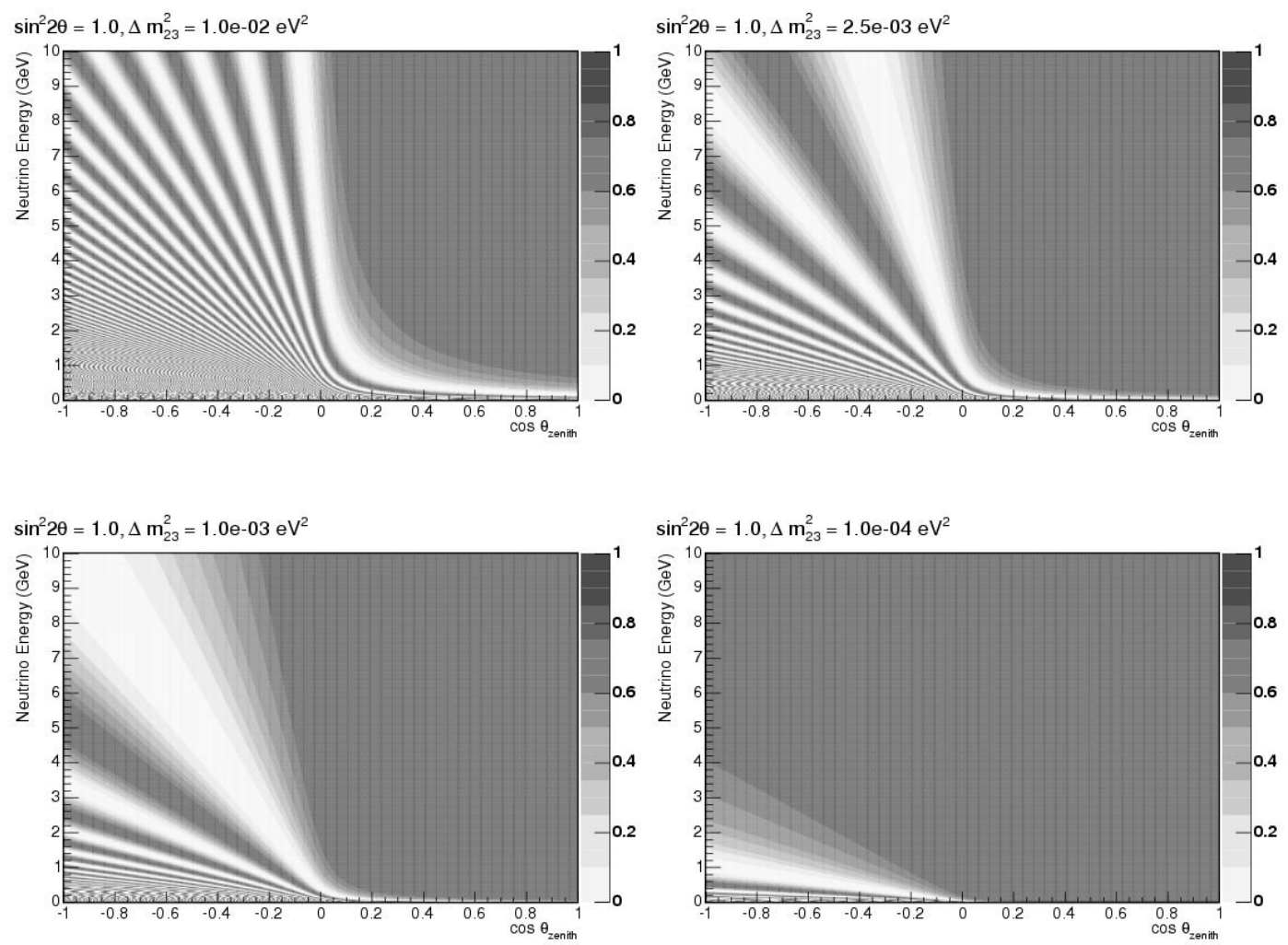

Figure 2.9: The atmospheric $\nu_{\mu}$ survival probability as a function of cosine of the zenith angle and the neutrino energy for four different values of $\Delta m_{23}^{2}$ assuming maximal mixing.

\subsubsection{General Features of Atmospheric Neutrino Experiments.}

While the atmospheric neutrino experiments vary in terms of detector technology they share some common features. The neutrinos are detected via the charged current 
interaction,

$$
\begin{gathered}
\nu_{e}\left(\bar{\nu}_{e}\right)+n(p) \rightarrow e^{\mp}+p(n) \\
\nu_{\mu}\left(\bar{\nu}_{\mu}\right)+n(p) \rightarrow \mu^{\mp}+p(n) .
\end{gathered}
$$

The observed charged lepton is used to estimate the direction and energy of the parent neutrino.

The candidate neutrino events are commonly separated into three categories.

- Fully contained (FC) events. Neutrinos that interact inside the detector's fiducial volume and the lepton produced does not exit the detector. The lepton's momentum can be measured from the track's range.

- Partially contained (PC) events. Neutrinos that interact inside the detector's fiducial volume and the lepton produced exits the detector.

- Upward-going muons. Neutrinos that interact in the rock under the detector, so that only the muon produced is observed. Downward-going neutrino induced muons are impossible to distinguish from the much larger background of cosmic ray muons.

The dependance of the neutrino flux on the neutrino energy and zenith angle is then examined for evidence of neutrino oscillations.

\subsubsection{Atmospheric Neutrino Experiments}

The predictions of the total flux of neutrinos are estimated to carry a $20 \%$ uncertainty (discussed in Chapter 5) dominated by the uncertainty in the primary cosmic ray spectrum and in the hadronic production models. However, the relative flux of muon to electron type neutrinos carries an uncertainly of just 5\%. By examining Equation 2.15, the expected ratio, $\mathcal{R}=N_{\nu_{\mu}} / N_{\nu_{e}}$, should be approximately 2 . The IMB experiment [24], a water Cerenkov experiment, was the first to measure this ratio and found a significant reduction compared to the expectation. This was later confirmed by the Kamiokande experiment [25], another water Čerenkov experiment, which saw a deficit of $30 \%$ in muon neutrinos. However, two early iron calorimeter experiments Fréjus [26] and NUSEX [27] observed no evidence of a deficit. The statistical significance of these early experiments was far from compelling.

The first compelling evidence that the reduction of the atmospheric neutrino flux is caused by neutrino oscillations was presented by the Super-Kamiokande experiment in 1998 [10]. The Super-Kamiokande experiment showed a clear dependance 
of the $\nu_{\mu} / \nu_{e}$ ratio on zenith angle. The latest zenith angle distributions from SuperKamiokande are shown in Figure 2.10.
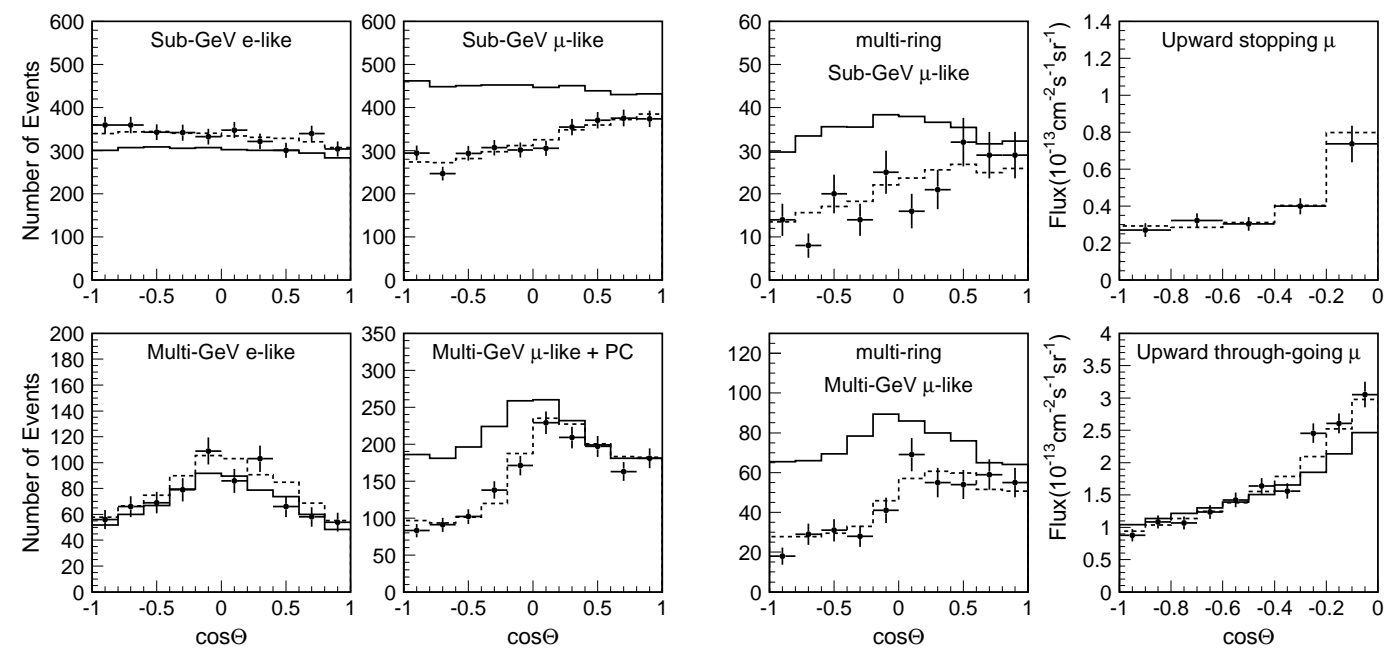

Figure 2.10: The zenith angle distribution from Super-Kamiokande's latest results [28]. Points show data for an exposure of 96 kton-years, the solid lines show the Monte Carlo expectation without neutrino oscillations and the dotted lines show the Monte Carlo expectation for best-fit to $\nu_{\mu} \rightarrow \nu_{\tau}$ oscillations.

By comparing the zenith angle distributions to the Monte Carlo expectations for 2-flavour $\nu_{\mu} \leftrightarrow \nu_{\tau}$ oscillation, the confidence limits shown in Figure 2.11 are obtained. The best fit point is located at $\sin ^{2} 2 \theta=1.0, \Delta m^{2}=2.0 \times 10^{-3} \mathrm{eV}^{2}$ and the $90 \%$ confidence limits are $\sin ^{2} 2 \theta>0.90$ and $1.3 \times 10^{-3}<\Delta m^{2}<3.0 \times 10^{-3} \mathrm{eV}^{2}$.

The Soudan 2 experiment has confirmed the observation of atmospheric neutrino oscillations. Soudan 2 was a 1 kton iron tracking calorimeter located at a depth of 2070 meters-water-equivalent on the $27^{\text {th }}$ level of the Soudan Underground Mine State Park, Minnesota, USA. Soudan 2 measured the $L / E$ distribution for fully and partially contained electron and muon neutrino interactions [29]. The $L / E$ distributions obtained after a 7.36 kton-year exposure are shown in Figure 2.12. A clear deficit of muon neutrinos can be seen at high values of $L / E$. The confidence limits obtained for 2-flavour oscillations are shown in Figure 2.11. The MACRO experiment located at the Grand Sasso Laboratory has also confirmed the Super-Kamiokande results [30] and the confidence limits obtained are shown in Figure 2.11.

Super-Kamiokande was also able to partially resolve a dip in the $L / E$ distribution 


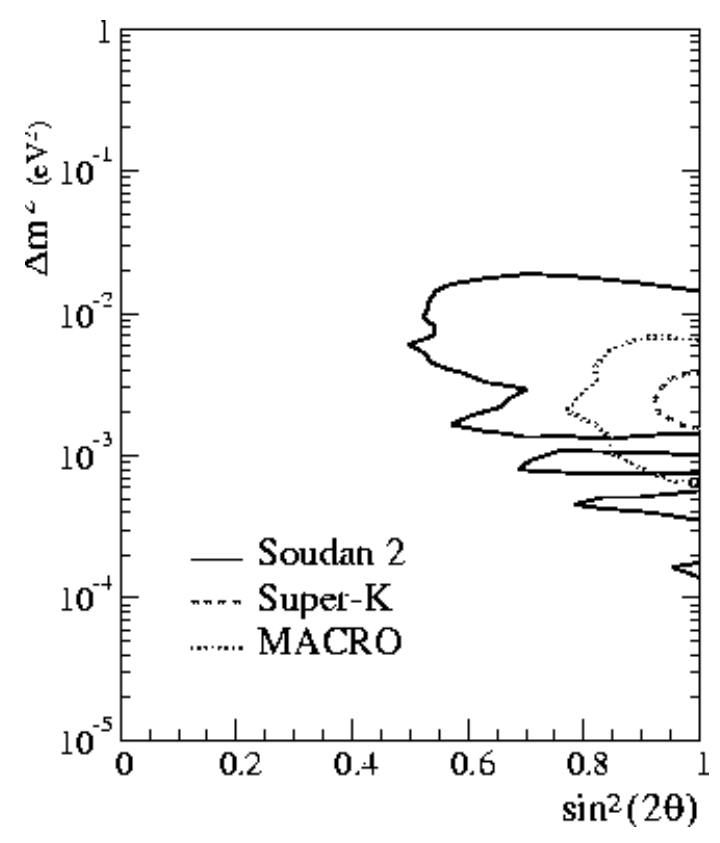

Figure 2.11: The allowed regions for atmospheric $\nu_{\mu} \leftrightarrow \nu_{\tau}$ neutrino oscillations. The $90 \%$ confidence limits are shown for the Super-Kamiokande, Soudan 2 and MACRO experiments. Figure taken from [29].

of their events by selecting events with high $L / E$ resolution [31]. The dip is characteristic of a sinusoidal neutrino oscillation probability and disfavours other models that give rise to zenith angle dependent neutrino disappearance such as neutrino decay [32] and neutrino decoherence [33]. The best fit to the neutrino decay hypothesis has a $\chi^{2}$ which is 3.4 standard deviations larger than the neutrino oscillation hypothesis and neutrino decoherence has a $\chi^{2} 3.8$ standard deviations larger. The probability that neutrino decay could mimic neutrino oscillations is approximately $0.1 \%$, with decoherence even more unlikely.

\subsection{Long Baseline Accelerator Experiments}

Long baseline experiments are designed to probe neutrino oscillation parameters to very high precision. In general they consist of an accelerator producing a high energy beam of protons which then strike a target producing pions that decay to $\nu_{\mu}$. The beam is measured at a point close to its source by a 'near' detector, i.e. before the neutrinos have oscillated. The beam is then propagated over a large distance, typi- 

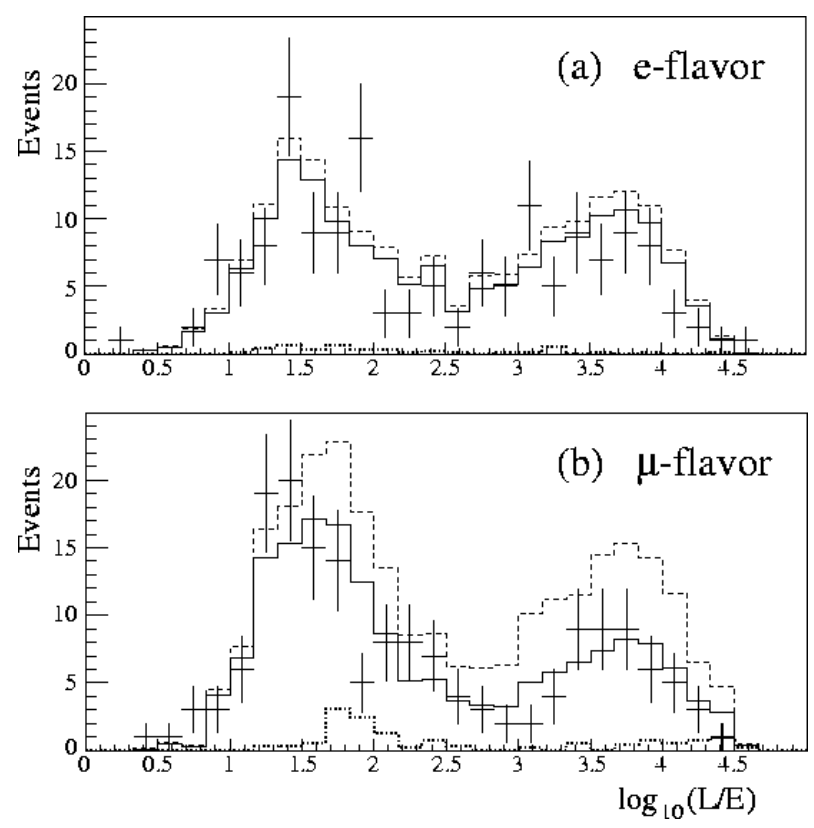

Figure 2.12: The $\log _{10}(L(\mathrm{~km}) / E(\mathrm{GeV}))$ for electron and muon type atmospheric neutrino events observed in the Soudan 2 detector for 7.36 kton-years exposure. Figure taken from [29].

cally hundreds of kilometres, to allow oscillations to develop and the beam spectrum measured again by a 'far' detector. Neutrino oscillations produce a characteristic dip in the ratio of the far and near energy spectra. The location of the dip gives the mass difference for the oscillations and the depth of the dip contains information about the mixing angle. The energy of the beam is tuned so that first oscillation minimum can be observed. For example, for $\Delta m_{23}^{2} \approx 0.0025 \mathrm{eV}^{2}$ and an experimental baseline of $700 \mathrm{~km}$, the ideal beam energy is $1.4 \mathrm{GeV}$. The near and far detectors are usually very similar in design to minimise the systematic errors in comparing near and far spectra.

The first generation of long baseline experiments have been designed to confirm and accurately measure the oscillation parameters associated with atmospheric neutrinos.

\subsubsection{The K2K Experiment}

The first long baseline experiment to start taking data was the KEK to Kamioka (K2K) experiment in Japan, which has a baseline of $250 \mathrm{~km}$ [34]. The K2K beam 
is $92 \% \nu_{\mu}$, peaked around $1 \mathrm{GeV}$ in energy and is directed towards the SuperKamiokande detector (described in Section 2.3.2). The beam is monitored by the near detectors which consist of a 1 kiloton water Cerenkov detector, which is essentially a scaled down version of the Super-Kamiokande detector, and a fine grained detector system. The predicted number of events and energy spectrum at the SuperKamiokande detector is estimated by measuring the beam at the near detectors and extrapolating to the far detector using Monte Carlo simulations. The number of events at the far detector is predicted using selected $\nu_{\mu}$ charged current events in the near water Čerenkov detector because they both have the same target material and uncertainties in the cross sections cancel. Fully contained accelerator events at Super-Kamiokande are selected based on timing information, the estimated backgrounds from atmospheric neutrinos is negligible. A total of 107 events were selected compared to the Monte Carlo prediction of $151_{-10}^{+12}$ in the absence of neutrino oscillations. The neutrino energy spectrum is estimated from the muon momentum and angle to the beam, assuming quasi-elastic kinematics. A sample of 57 single $\mu$-like ring events from the 107 events where selected and used to measure the beam spectrum at the far detector. The observed and predicted energy spectrum is shown in Figure 2.13. A clear distortion of the energy spectrum can be seen. A two-flavour neutrino oscillation analysis was performed, assuming $\nu_{\mu} \leftrightarrow \nu_{\tau}$ oscillations. Figure 2.14 shows the allowed regions obtained by K2K. They show excellent agreement with the results of the Super-Kamiokande atmospheric neutrino analysis.

The excellent flavour separation ability of the water-Čherenkov detectors allows $\mathrm{K} 2 \mathrm{~K}$ to do $\nu_{e}$ appearance studies [35]. No significant excess of $\nu_{e}$ events were observed. The low statistics of the study mean that the limit on electron mixing obtained is approximately half as sensitive as that obtained by Chooz.

\subsubsection{MINOS}

The Main Injector Neutrino Oscillation Search (MINOS) will begin taking data in January 2005. It consists of two similar magnetised iron-scintillator sampling calorimeter detectors separated by $734 \mathrm{~km}$ and a variable energy $\nu_{\mu}$ beam. The beam project is called NuMI (Neutrinos at the Main Injector).

The beam is produced using $120 \mathrm{GeV}$ protons from the Fermilab Main Injector located near Chicago, USA. The proton beam then impinges on a graphite target. The charged mesons produced ( predominantly $\pi^{ \pm}$and $K^{ \pm}$) are then focused by two magnetic horns. The two horns act like a pair of achromatic lenses. By adjusting the inter-horn distance, the energy spectrum of the focused mesons can be adjusted. 


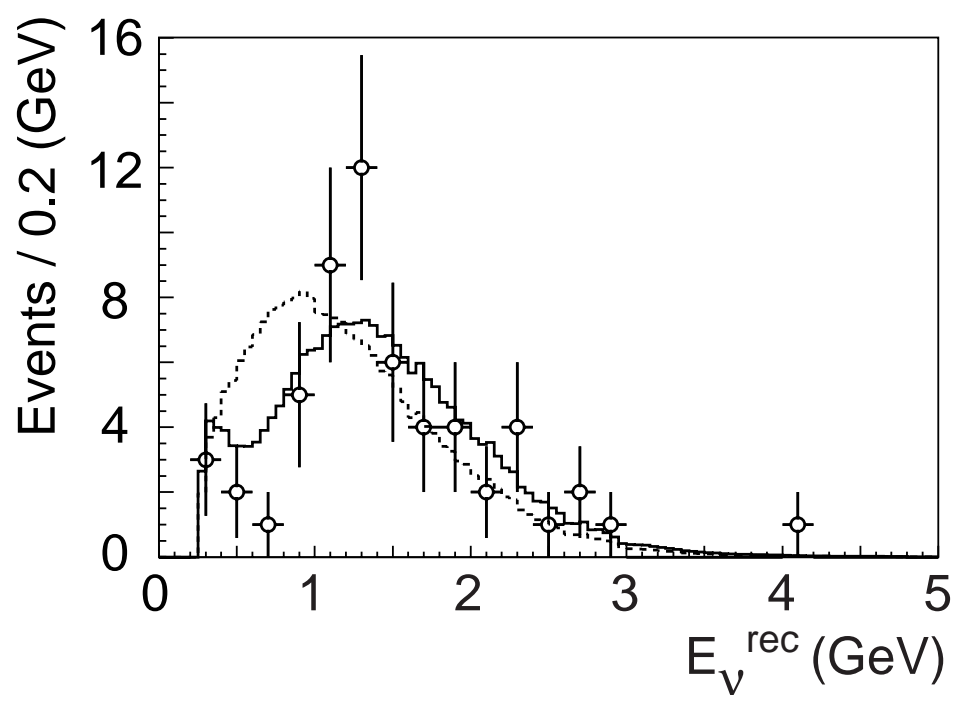

Figure 2.13: The reconstructed neutrino energy distribution for the single ring $\mu$-like sample measured by the K2K experiment. Points with error bars show the data, The solid line shows the Monte Carlo expectation for the best-fit solution to $\nu_{\mu} \rightarrow \nu_{\tau}$ oscillations. The dashed line is the expected spectrum in the absence of oscillations. Figure taken from [34].

The focused $\pi^{+}$and $K^{+}$then decay in a $675 \mathrm{~m}$ decay pipe producing muon neutrinos directed towards the Far Detector.

A near detector is located at the end of the decay tunnel and measures the beam energy spectrum. The measured energy spectrum at the near detector can then be extrapolated to the far detector and compared to the observed spectrum. While the atmospheric neutrino results are well described by a sinusoidal neutrino oscillation probability, there are alternative models. MINOS will be able to make a high statistics measurement of the oscillation probability (Figure 2.15) and make a conclusive statement about the alternative models for $\nu_{\mu}$ disappearance. MINOS will be able to make a high precision (10\%) measurement of $\Delta m_{23}^{2}$. Figure 2.15 shows the expected sensitivity compared to the Super-Kamiokande measurement for three different numbers of protons on target. MINOS will also search for subdominant $\nu_{\mu} \rightarrow \nu_{e}$ oscillations. The $3 \sigma$ discovery potential for $\theta_{13}$ is approximately a factor of two improvement on the current Chooz limit.

While the main goal of the MINOS experiment is analysis of the beam data, the MINOS Far Detector provides a unique opportunity to do a charge separated atmospheric neutrino oscillation analysis. This thesis details the first charge separated contained event results from MINOS. 


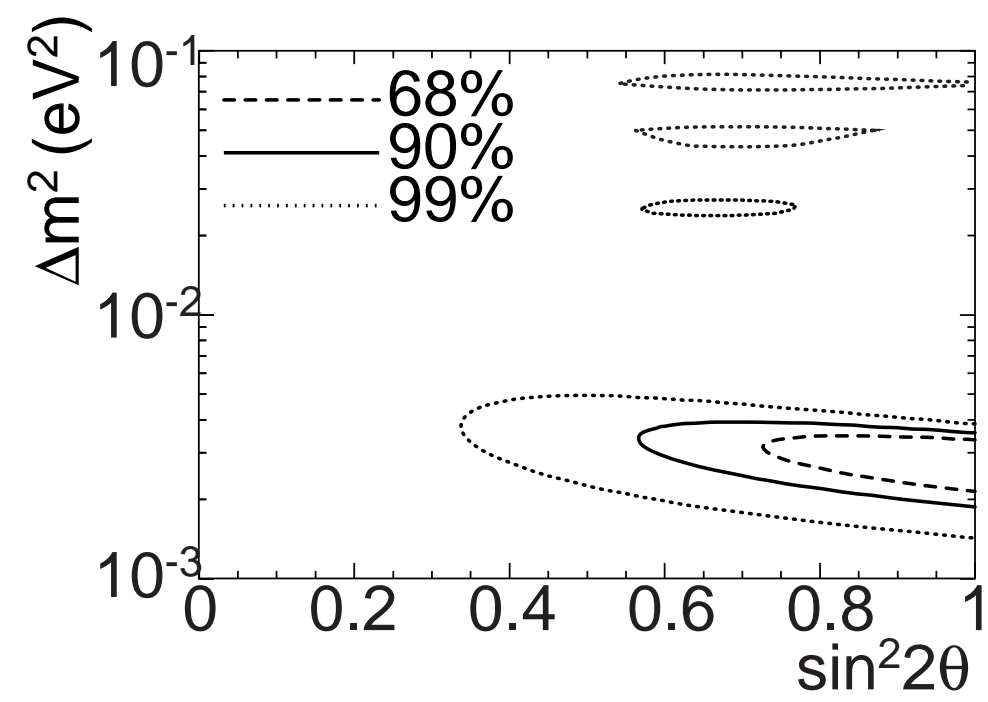

Figure 2.14: The allowed oscillation parameter regions as obtained by the K2K experiment, the lines show different confidence limit contours. Figure taken from [34]

\subsubsection{CNGS}

The CERN to Gran Sasso (CNGS) [36] project is another long baseline experiment located in Europe. The CNGS uses a multi-GeV $\nu_{\mu}$ beam created at CERN and directed towards the Gran Sasso Laboratory in Italy, $732 \mathrm{~km}$ away. Two experiments are located at Gran Sasso, Oscillation Project with Emulsion Racking Apparatus (OPERA) and Imaging Cosmic And Rare Underground Signals (ICARUS). Both experiments are designed to detect the appearance of $\nu_{\tau}$ in the CNGS beam.

\section{$2.7 \quad$ LSND}

The Liquid Scintillator Neutrino Detector (LSND) experiment was a short baseline experiment (approximately $30 \mathrm{~m}$ ) located at Los Alamos, USA. It reported the appearance of $\bar{\nu}_{e}$ in a beam of $\bar{\nu}_{\mu}, 4$ standard deviations above the expected background [37]. The energy and baseline of LSND implies $\Delta m^{2} \sim 1 \mathrm{eV}^{2}$. As there are only three neutrino flavours, there can only be two independent mass differences. The LSND result is therefore incompatible with the results of solar and atmospheric neutrino experiments. There have been several speculative solutions to the LSND anomaly proposed [38], including the existence of a sterile neutrino that does not interact weakly and that $C P T$ may be violated giving a different set of masses for 

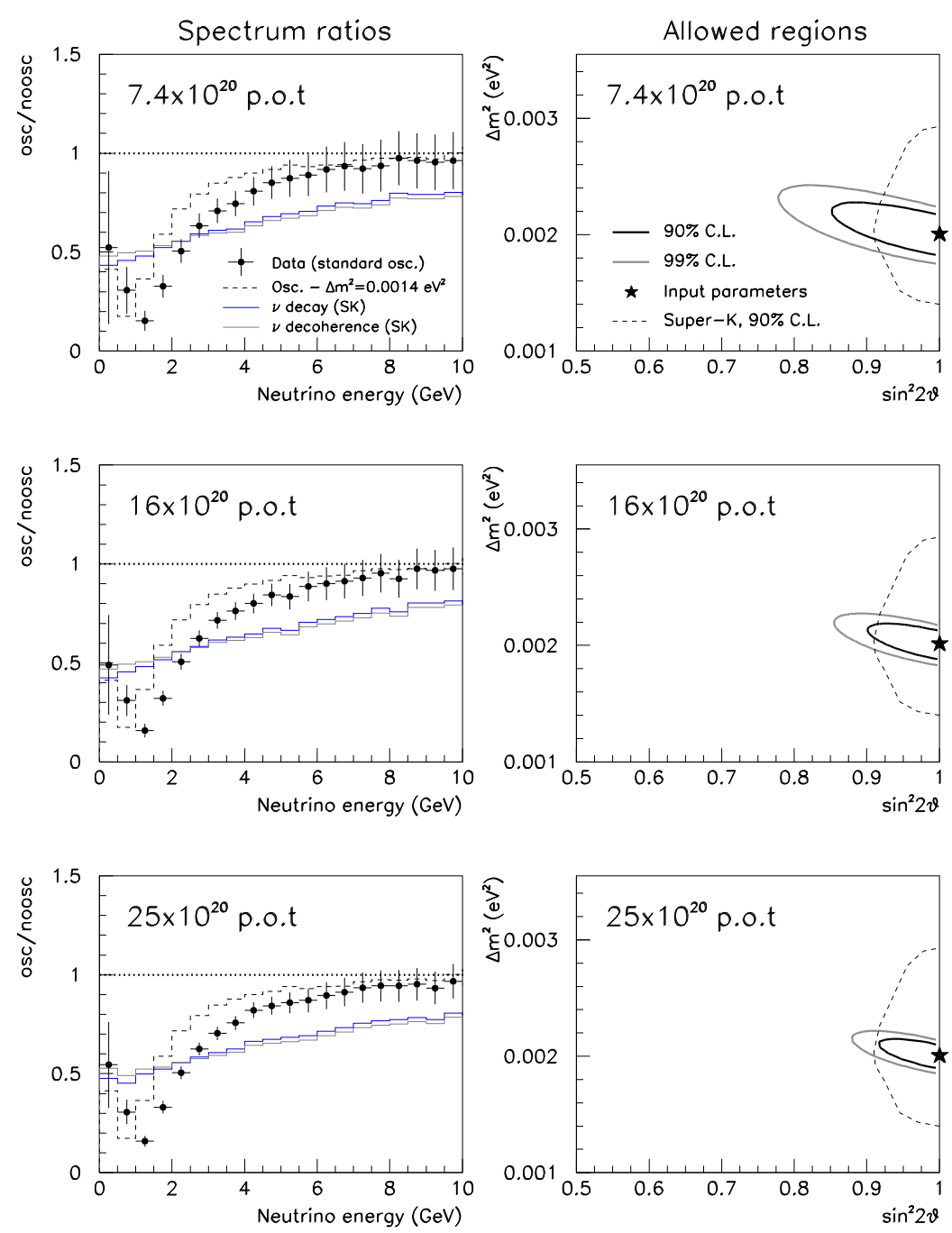

Figure 2.15: (Left): The expected ratio, for 3 difference exposures, of the far to near energy spectrum in the MINOS detectors for oscillation parameters $\Delta m_{23}^{2}=$ $0.002 \mathrm{eV}^{2}$ and $\sin ^{2} 2 \theta_{23}=1.0$. (Right): The corresponding expected $90 \%$ confidence limits compared to the limit obtained by Super-Kamiokande.

neutrinos and anti-neutrinos. However, the SNO results have heavily restricted the contribution of a sterile neutrino and the violation of $C P T$ in the neutrino sector looks increasing unlikely [39]. In addition the KARMEN [40] and NOMAD [41] experiments have excluded much of the parameter space allowed by LSND.

The MiniBooNE experiment 42 at Fermilab is attempting to confirm the LSND results. MiniBooNE is looking for $\nu_{\mu}$ to $\nu_{e}$ oscillations and is able to exclude oscillations by at least $5 \sigma$ if not in the LSND allowed regions. 


\subsection{Summary}

Since the first observation of the solar neutrino deficit, the SNO and KamLAND experiments have provided compelling evidence that this deficit is indeed due the oscillation of electron neutrinos. The evidence for atmospheric neutrino oscillations from Super-Kamiokande has been confirmed by other experiments and by an accelerator experiment.

If it is assumed that the coupling between the atmospheric and solar sectors is small, as suggested by the Chooz experiment, then we can identify the mass splittings as $\Delta m_{\odot}^{2}=\Delta m_{12}^{2} \approx 8 \times 10^{-5} \mathrm{eV}^{2}$ and $\Delta m_{\text {atmos }}^{2}=\Delta m_{23}^{2} \approx \Delta m_{13}^{2} \approx 2.5 \times 10^{-3} \mathrm{eV}^{2}$. The results of the atmospheric neutrino experiments strongly suggest near maximal mixing, $\sin ^{2} 2 \theta_{23} \approx 1.0$ or $\theta_{23} \approx 45^{\circ}$. The combination of the solar neutrino and KamLAND results suggest $\tan ^{2} \theta_{12}=0.4$, or $\theta_{12} \approx 30^{\circ}$. Therefore, in the limit of $\theta_{13}=0, U$ can be written as

$$
U \approx\left(\begin{array}{ccc}
\frac{\sqrt{3}}{2} & \frac{1}{2} & 0 \\
-\frac{1}{2 \sqrt{2}} & \frac{\sqrt{3}}{2 \sqrt{2}} & \frac{1}{\sqrt{2}} \\
\frac{1}{2 \sqrt{2}} & -\frac{\sqrt{3}}{2 \sqrt{2}} & \frac{1}{\sqrt{2}}
\end{array}\right)
$$

The results from the solar neutrino experiments have resolved the sign ambiguity of $\Delta m_{12}^{2}$, but the sign of $\Delta m_{23}^{2}$ is still unresolved. However, if $\theta_{13}$ is non-zero, matter effects would be introduced in atmospheric neutrinos, which would be seen in $\nu_{\mu}$ but not $\bar{\nu}_{\mu}$. It has been suggested [43] that an atmospheric neutrino detector that is capable of distinguishing the $\nu_{\mu}$ charge, such as MINOS, may be able to resolve the ambiguity.

The MINOS atmospheric neutrino analysis will measure the oscillation parameters for $\nu_{\mu}$ and $\bar{\nu}_{\mu}$ and will place strong constraints on $C P T$ violating models that have been suggested to accommodate the LSND and atmospheric neutrino results. 


\section{Chapter 3}

\section{The MINOS Far Detector}

A brief overview of the MINOS Far Detector is given. Particular attention is paid to the veto shield which is central to the physics analysis presented in this thesis. A detailed discussion of the Data Acquisition System is given in the following chapter.

\subsection{Detector Physical Overview}

The Far Detector is located on the 27th level of the Soudan Underground Mine State Park, Soudan, Minnesota, USA. The laboratory is situated at a depth of $714 \mathrm{~m}$, or 2100 metres-water-equivalent (mwe), giving a $10^{5}$ reduction in cosmic ray muon rates. The MINOS collaboration share the laboratory with CDMS 44] and the recently decommissioned Soudan 2 experiment [45]. The design of the detector was constrained by the size and capacity of the elevator which serves as the only access point to the mine. A schematic of the Far Detector is shown in Figure 3.1 and a photograph of the completed detector is shown in Figure 3.2 .

The Far Detector is a steel-scintillator sampling calorimeter with a total mass of 5.4 kilotons. It is composed of 483 active planes each consisting of an octagonal steel plate 8 meters across, $2.52 \mathrm{~cm}$ thick and backed with 192 scintillator strips. Each strip is $4.1 \mathrm{~cm}$ wide, $1 \mathrm{~cm}$ deep and runs the full width of the plane. Strips are optically isolated from their neighbours by a plastic coating and are grouped together into modules of 20 or 28 strips. The modules are covered by a light tight aluminium casing and are welded onto the steel planes. A plane is called a "U-plane" or "Vplane" depending on the orientation of its strips. Adjacent planes have orthogonal orientations.

The planes are arranged to be approximately perpendicular to the NuMI beam 


\section{East}

\section{West}

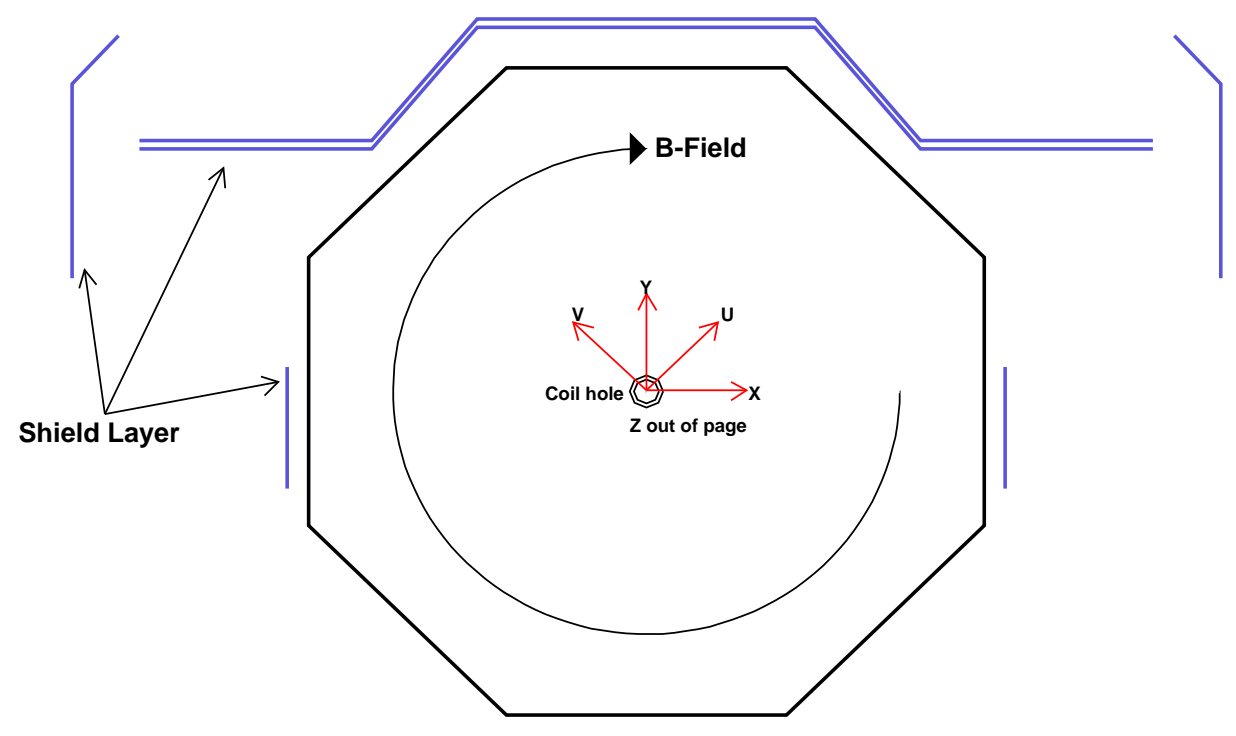

Figure 3.1: A schematic of the Far Detector in the $X Y$ view. The co-ordinate system used is shown. The veto shield layers are shown in blue.

direction and are separated by a $1 \mathrm{~cm}$ air gap. The detector is separated into two sub-detectors, called Supermodules separated by a $1.1 \mathrm{~m}$ gap. Supermodule 1, which is the most southern of the two, has 248 steel planes and Supermodule 2 has 236. Each Supermodule is magnetised by a $15 \mathrm{kA}$ coil that runs perpendicularly through the centre of the planes and returns below the detector. The coils generates a toroidal magnetic field in the steel with a mean strength of 1.5 Tesla.

Scintillation light generated by the passage of a charged particle is collected by a wavelength shifting (WLS) fibre glued into one side of the strip. The light signal is propagated to both ends of the strip where the WLS fibre is coupled to long clear fibre cable which carries the signal to 16-pixel Hamamatsu R5900-00-M16 photomultiplier tubes (PMT) up to several meters away. Each PMT has a sixteen pixel anode arranged in a $4 \mathrm{~mm} \times 4 \mathrm{~mm}$ grid and a set of twelve common dynodes. At the PMT's face, signals from eight strips in a plane are optically summed, or multiplexed, into one pixel of the PMT. The multiplexing introduces ambiguities in the hit location. 


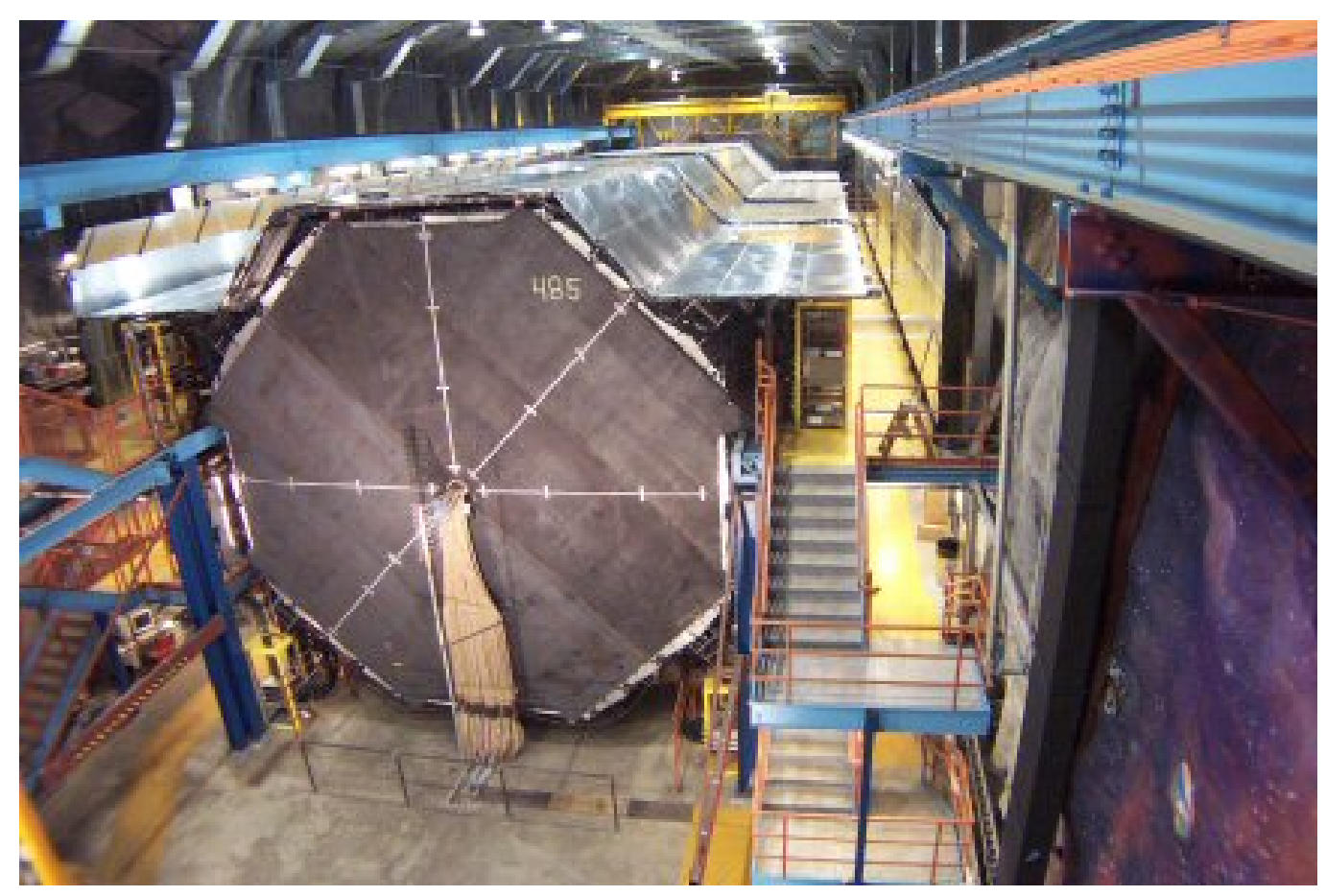

Figure 3.2: The completed Far Detector viewed from the north looking south.

\subsection{Far Detector Front-End Electronics}

Three photomultipliers are arranged inside a light-tight $M U X$ box. Each MUX box has a VA Front-end Board (VFB) which provides analogue readout and trigger information for the three photomultipliers. An Amplifier/Shaper/Discriminator chip (ASD-lite) triggers readout of the photomultipliers based on the charge on the last dynode stage. Three VA chips, from IDEAS of Oslo, provide analogue readout of the photomultiplier anodes. A VA chip can shape, sample and hold all 16 channels of a photomultiplier synchronously.

Each VFB is a slave module and is under the direct control of a VA Readout Controller (VARC), which co-ordinates readout for up to 12 VFBs (36 VA chips). The VARCs are situated in the DAQ crates located several meters away from the MUX boxes. Each VARC has six VARC Mezzanine Modules (VMM) handling the digitisation and readout co-ordination of two VFBs (six VA chips). Each VMM has one Analogue-to-Digital Converter (ADC) for all the chips it handles.

A dynode trigger signal is time-stamped and after a short delay (approximately $200 \mathrm{~ns}$ ) to allow the peaking of the signal, the VA chip is sent the sample and hold 

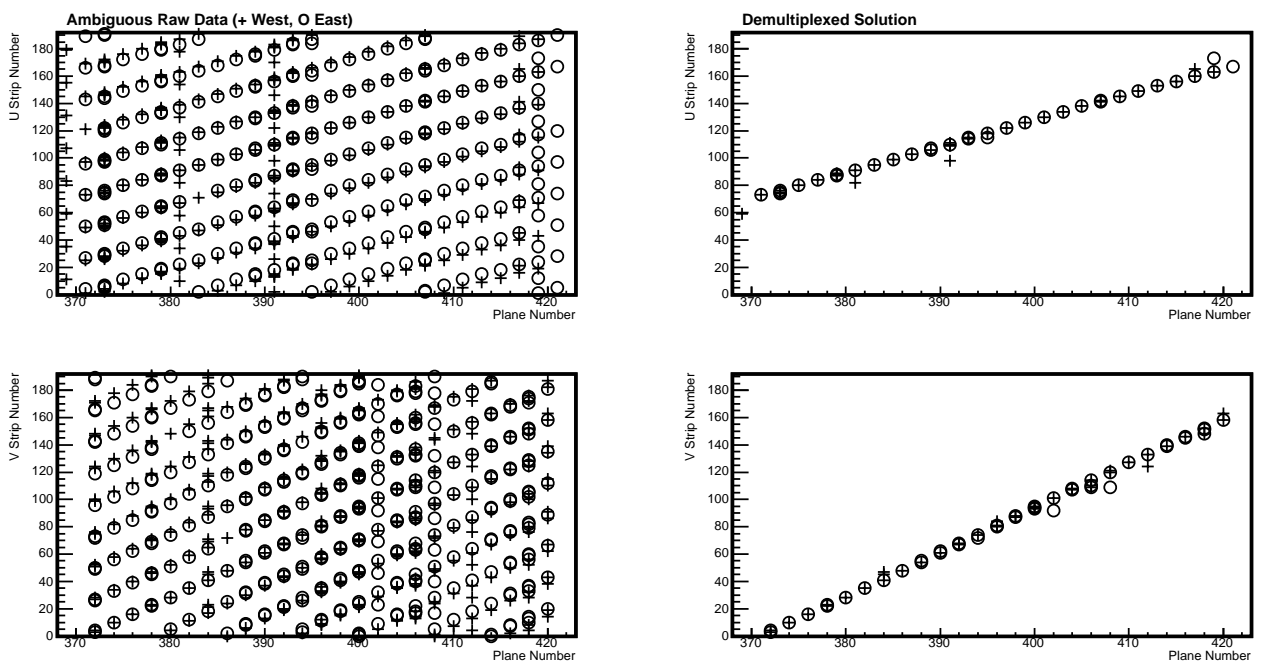

Figure 3.3: A cosmic ray muon passing through the detector. The left panels show the hit location ambiguity due to optical multiplexing. The location of the muon hit on each plane can be determined by point at which the strip alternatives from each side coincide. The right hand panels show the event after the demultiplexing software has been run.

command. The trigger is then placed in a queue to be digitised by the VMM's ADC. As the digitisation process takes $5 \mu s$ and only one of the six chips under the control of the VMM can be digitised at a time, it can be up to $30 \mu s$ between a chip receiving the sample and hold command and the trigger being digitised, during which time further dynode triggers are ignored. This is the major source of dead time in the detector.

Once the trigger has been digitised it is transferred to the VARC's Sparsifier which performs three duties; pedestal subtraction, common noise subtraction and sparsification. Each channel has an inherent offset, or 'pedestal', which is calculated during special calibration runs and subtracted from each channel reading during normal data-taking. It has been found that the some of the inherent noise in the system effects all channels on a single VA chip equally, this is called common mode noise. The Sparsifier removes common mode noise by subtracting readings from channels not attached to photomultiplier pixels. The last step is data sparsification which is the removal from the data stream of all channel readings below a threshold of $1 / 5$ of a photoelectron [46]. The data is then transferred to the VARC output buffers for collection by the Data Acquisition System (DAQ). The DAQ collects data 
from all the VARCs and forms physics events.

In response to the higher than predicted noise rates, a 2 out of 36 trigger has been implemented in the VARC. For data from a VARC to be readout, two out of a possible total of 36 VA chips on the VARC must be triggered within 500 ns of each other.

\subsection{Event Information}

For each physics event, a series of 'digits' is recorded. Each digit contains an electronics channel number, a pulse height and a time-stamp. The electronics channel number is used to identify which PMT pixel recorded the hit. Due to the optical multiplexing, there are eight possible strips that might have given rise to the signal. However, as the multiplexing pattern is different on the west and east sides of the detector, the ambiguity can be removed in software by examining the overlap of possible strips on each side. Figure 3.3 shows the ambiguity in the hit location and the demultiplexed solution. The two strip orientations give two views of the event, $U-Z$ and $V-Z$ which can are combined to form three dimensional tracks and showers. Figure 3.4 shows an example of an event display for a through-going cosmic ray muon event after demultiplexing. The direction of the track can be clearly determined from the timing of hits along its length. Once tracks have been identified, the curvature of the track in the magnetic field is used to identify the charge and momentum of the particle that produced it. The digit pulse height is used to estimate the energy deposition in each strip from hadronic and electromagnetic showers.

\subsection{Calibration Overview}

\subsubsection{Energy Calibration}

Measuring the energy of a $\nu_{\mu}$ charged current event in a MINOS detector requires measuring the momentum of the muon and the energies of hadronic or electromagnetic showers. The muon momentum is measured either from range or curvature in the magnetic field. This depends on the track topology and not on the energy deposited in each hit, it is therefore not sensitive to the strip response calibration. However, the response of the scintillator strips to hadronic showers must be well understood.

The gain and linearity of the PMTs are measured and monitored using a light injection system. Light from UV Light Emitting Diodes (LED) is injected into the 

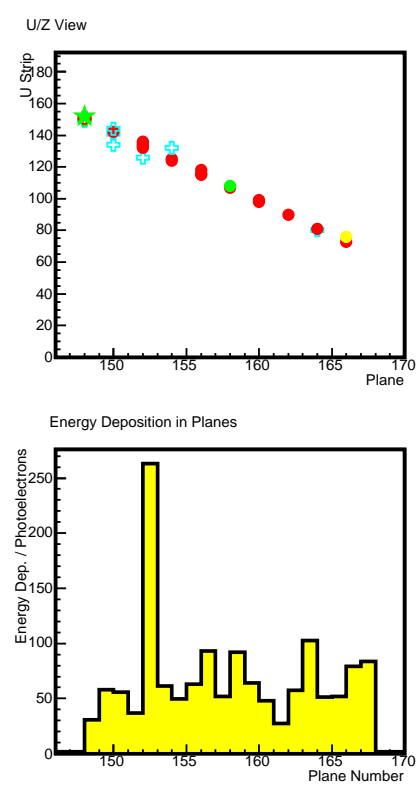

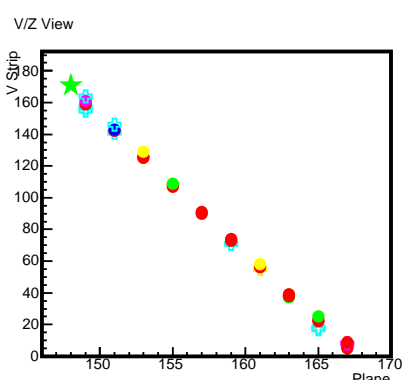

Run : 18141 Snarl: 500 Trk: 1 Shw: 1
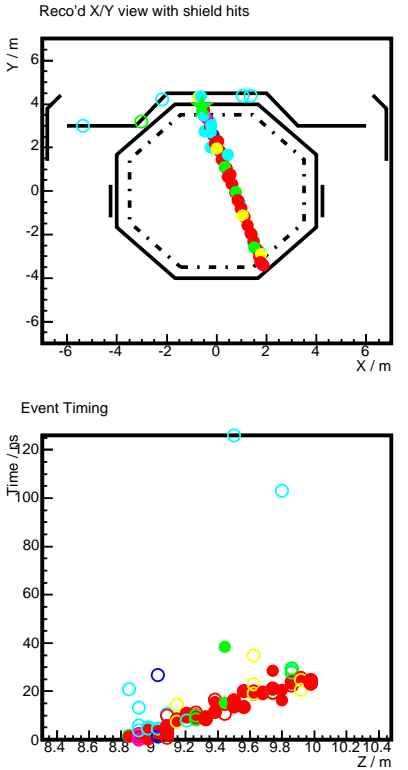

Figure 3.4: A cosmic ray muon passing through the detector. The top left two panels shows the raw data from both views after demultiplexing. The top right is the $X / Y$ reconstruction from the two views. The $X / Y$ view also shows the hits in the veto shield, closed circles are in-time veto shield hits, open circles are out-of-time. The bottom left shows the energy deposited in each plane calibrated to remove the electronics variation but not the differences in strip response or attenuation in the fibres. The bottom right shows the time of hits against $Z$, from which the direction of the muon can be easily determined (closed circles are hits associated with the track).

wavelength shifting fibre at the end of the strips. The signal measured by the PMT is compared to a PIN diode which is flashed with the same pulse that illuminates the fibres and is read out in a spare channel on the front-end electronics. The stability of the PIN diodes has been shown to be better than $0.5 \%$ over a period of two months [47. The light injection system performs three tasks:

- Gain Mapping. The gain of the PMTs is highly non-linear above 100 photoelectrons. The light injection system is used to map the gain curve by flashing the strips at a range of light levels and comparing the PIN readout with the PMT ADC values. The non-linearity in ADC conversion is removed by a special calibration run where a series of known charges are injected into the channels of the VA chips.

- Drift Monitoring. The gain of a PMT will vary as a function of time due to 
environmental and supply voltage changes. By pulsing a test point in the gain curve the light injection system can create calibration constants to correct for short term variations in the gain curve.

- Optical Integrity. The light injection system is used to check that the optical pathways are working.

To maximise live-time and the frequency of calibration, light is injected in parallel with physics data-taking. To identify light injection events in the data a dedicated PMT, the TriggerPMT (TPMT), is flashed in time with injection of light into the detector. The Trigger Farm uses hits from the TPMT to identify and remove light injection events from the physics data stream.

Once the variation in electronics and PMT gains have been corrected for, the variation in strip response must be calibrated. The strip response varies with scintillator light output, fibre collection efficiencies and fibre attenuation constants. While these effects have been measured for each component during construction, it is desirable to have a strip-to-strip calibration measured simultaneously and in situ with data-taking. Cosmic ray muons provide an excellent test beam for strip response calibration. The response of each strip is measured in MIPs. One MIP is defined to be the energy deposited in one strip by a minimum ionising muon travelling at normal incidence to the planes.

To obtain the hadronic energy scale, a small calibration detector was placed in a test beam at CERN. The response of the detector to hadrons of known energy relative to muons was measured. The relative response to muons between the calibration and Far Detector can then be used to obtain the hadronic response of each strip.

\subsubsection{Timing Calibration}

The timing resolution of a single hit is dominated by four processes.

- Clock resolution. The time of dynode triggers is time stamped by a $640 \mathrm{MHz}$ clock giving a minimum timing resolution $0.45 \mathrm{~ns}$. This provides a hard limit in the timing resolution.

- Single trigger per PMT. The time-stamp for a hit is set to the dynode trigger time. However, all pixels on a PMT share the same dynode trigger. Therefore, the time-stamp of any PMT hit is the time of the first dynode trigger. 
- Electronics and PMT. The electronics and PMTs introduce several delays due to signal propagation and pulse shapes. These delays depend on the pulse height of the signal, an effect known as time-walk.

- Strip Response The rise and decay time of light signals in the fibres.

The first two sources provide a hard limit on the timing resolution. However, the second two can be calibrated out by looking at the timing residuals for cosmic ray muon tracks in the detector. If it is assumed that the muon is travelling at $c$ then the residuals to a fit for recorded time against path length gives the timing calibrations for each strip. After calibration, the mean strip timing resolution is $2.6 \mathrm{~ns}$.

\subsection{The Veto Shield}

\subsubsection{Veto Shield Overview.}

The surface area of the detector sides is $40 \%$ air gaps, $40 \%$ steel and $20 \%$ scintillator. Therefore, $80 \%$ of the surface area is uninstrumented. A veto shield covering the top and sides if the detector has been constructed to tag charged particles entering the detector. It is constructed using the same scintillator modules used in the detector and is read out using the same front-end electronics and DAQ. However, the chips that read out the veto shield are not included in the 2-out-of-36 VARC trigger.

The scintillator strips are orientated along the $Z$ axis of the detector and read out at both ends. Each supermodule is covered by two shield sections. As each section is $8 \mathrm{~m}$ long and each supermodule is approximately $15 \mathrm{~m}$ in length, the two sections overlap by about a mere in the centre of the supermodule. The shield that covers the top of the detector has two layers of scintillator and the wall modules use one, as shown in Figure 3.1. The cosmic ray muon acceptance of the shield calculated using Monte Carlo is shown in Figure 3.5. Of the cosmic ray muons that deposit energy in eight or more planes and do not enter the detector via the Supermodule ends, less than $0.1 \%$ do not intercept the shield. Figure 3.5 shows the veto shield acceptance for cosmic ray muons in terns of the angle to the $Y$ axis in the XY plane and the intercept to that axis. The coloured histogram shows the parameter space occupied by the downward going cosmic ray muons as calculated from Monte Carlo. Cosmic ray muons below the black lines hit the detector. Cosmic ray muons below the red lines intercept at least one layer of shield. The two regions of shield acceptance correspond to the upper and lower wing sections. 


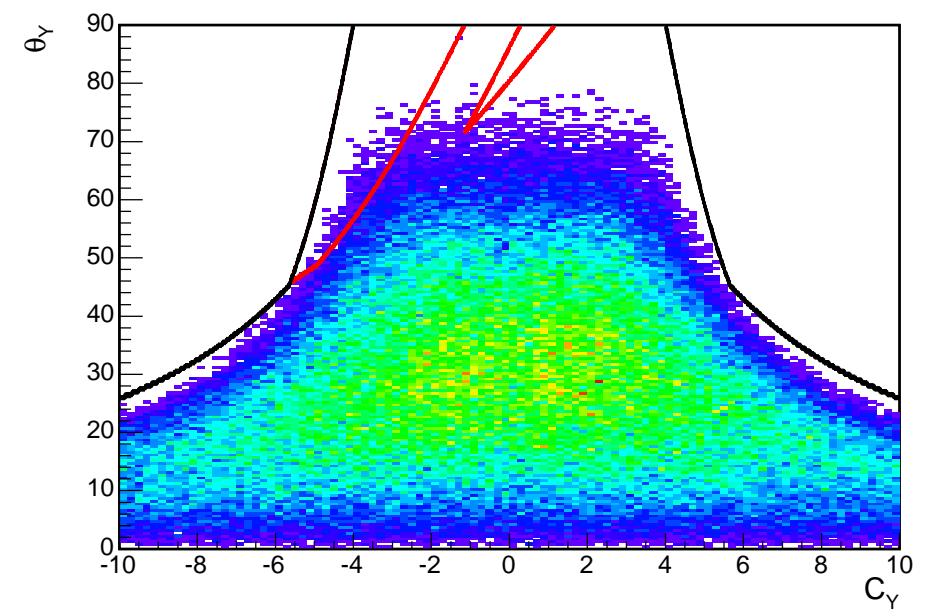

Figure 3.5: True muon angle to the $Y$ axis in the $X Y$ plane, $\theta_{Y}$, against the true intercept of the muon trajectory on the $Y$ axis, $C_{Y}$, for muons that deposit energy in 8 or more planes and do not cross the first or last 5 planes of the detector. The detector acceptance is shown by the black line: muons with parameters above these lines miss the detector. The shield acceptance is shown by the red line: muons with parameters below this line cross at least one layer of veto shield scintillator.

The read out from eight adjacent strips is multiplexed together to form a plank which is read out by a single electronics channel on each end. It should be noted that, as the multiplexing pattern is the same on both ends, it is not possible to demultiplex the shield as it is in the main detector. Therefore, the hit location resolution of the shield in the $X Y$-plane is approximately $10 \mathrm{~cm}$.

\subsubsection{Shield Noise}

There are three sources of noise in the veto shield.

- $\gamma$ Radiation. The dominant sources of $\gamma$ radiation in the MINOS hall are radon gas, $U^{238}, T h^{232}$ and $K^{40}$ decays. The singles rate from $\gamma$ radiation has been estimated to be $350 \mathrm{~Hz} / \mathrm{m}^{2}$ [48]. The effect of radon gas can be seen in the variation of noise rates with season shown in Figure 3.6 .

- PMT Dark Noise. The PMTs are noisy due to thermal emission of electrons from the photo-cathode resulting in a noise spectrum peaked at a single photoelectron. There is a small component of noise from thermal emission from the 
first dynode. The dark noise rate has been measured and varies between 0.5 and $1 \mathrm{kHz}$ for a threshold corresponding to $1 / 3$ of a photoelectron.

- Fibre Noise. The wavelength shifting fibre has been shown to be a significant source of noise, about $100 \mathrm{~Hz}$ per meter of fibre [49]. The source of this noise is believed to be relaxation of mechanical stress created when gluing the fibres into the scintillator strips. The fibre noise is decreasing with time with a decay constant of the order of 100 days.

To determine the singles rate in the shield, special runs without the DAQ triggers were taken. The single and double-ended rates for each shield section are presented in Table 3.1. A double-ended hit is required to have charge on both ends of a plank within $100 \mathrm{~ns}$. The mean rate of dynode triggers in each shield PMT is monitored by the DAQ and written to file.

\begin{tabular}{|c|c|c|}
\hline Section Number & Singles Rate $/ \mathrm{kHz}$ & Double Ended Rate $/ \mathrm{kHz}$ \\
\hline 1 & 130.1 & 13.9 \\
\hline 2 & 129.5 & 13.7 \\
\hline 3 & 108.3 & 9.3 \\
\hline 4 & 123.1 & 11.6 \\
\hline
\end{tabular}

Table 3.1: The mean single and double ended noise rates in the veto shield, for an example point during the data-taking.

\subsubsection{Tuning the PMT Gains}

The shield does not have a light injection calibration system. Therefore, it is not possible to use the method of gain calibration used in the main detector. However, the single photoelectron peak can be used to estimate the gain for each PMT pixel. The dynode trigger threshold on the shield PMTs was reduced to $1 / 3$ of a photoelectron and 1 minute of untriggered shield data was taken. Figure 3.7(a) shows the distribution of signals for an example PMT pixel in ADC counts. The distribution is dominated by the single photoelectron peak. The central peak was fitted to a Gaussian distribution to obtain an ADC count to photoelectrons conversion for each channel. The high voltage on each PMT was then adjusted to minimise the spread of PMT gains across the shield. Figure 3.7(b) shows the gain of the shield PMT pixels after the high voltage adjustments. The mean gain of the shield PMTs is $89.8 \pm 10.8$ ADC counts per photoelectron. 


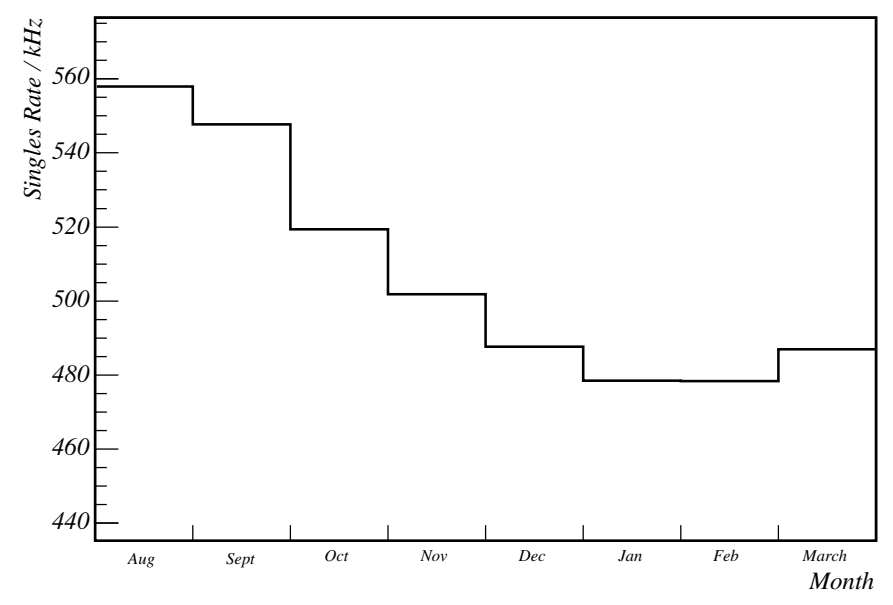

Figure 3.6: The total rate of dynode triggers in the veto shield over the period of data taking, the seasonal effect of radon gas concentrations can be observed.

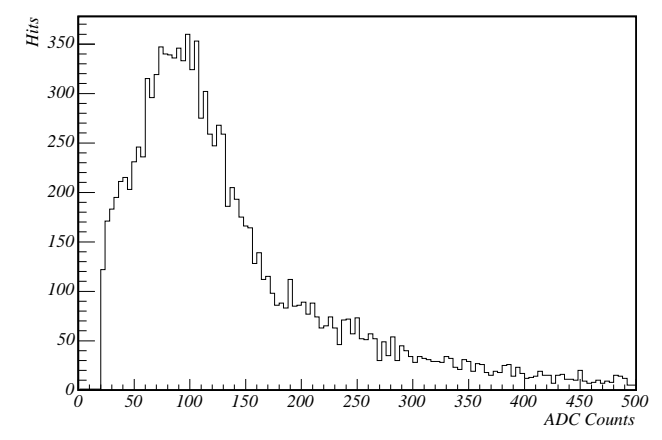

(a)

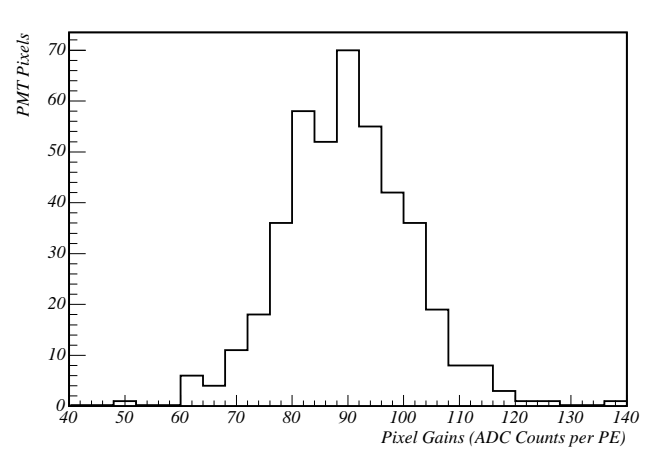

(b)

Figure 3.7: (a) An example of the single photoelectron peak for a single PMT. (b) The distribution of gain constants for the veto shield after tuning of high voltages.

\subsubsection{Shield Timing}

To correctly associate events in the detector with veto shield hits the timing resolution of the veto shield must be understood. Cosmic ray muons that pass through the detector were selected from the data. The muon track was required to be a good fit to a straight line in both $U Z$ and $V Z$ views. The rate of such events is $0.14 \mathrm{~Hz}$. The 
muon was projected back to the shield and required to intercept at least one layer.

For each event the 'golden' shield hits are selected. Golden shield hits are hits that are clearly caused by the muon. For a shield hit to be golden it must pass two requirements.

1. It must be double ended and have a total charge, before attenuation correction, of greater than 5 photoelectrons.

2. The angle in the $X Y$-plane between the muon trajectory and the line joining the point the muon entered the detector with the centre of the hit plank be less than $30^{\circ}$.

The estimated time that the muon crossed the shield, $t_{m}$, is calculated by subtracting the travel time between the muon entry point and the projected shield intercept point. The difference between $t_{m}$ and the muon crossing time estimated from the veto shield hit $t_{v}, \Delta t_{0}$ is shown in Figure 3.8. The mean value of $\Delta t_{0}$ is calculated for each plank, $\overline{\Delta t_{0}}$. The $\overline{\Delta t_{0}}$ s are then used as timing calibration constants for the shield. The calibration constants are shown in Figure 3.9. The timing resolution before and after application of the timing calibrations is shown in Figure 3.8. The widths of the $\Delta t_{0}$ distributions before and after calibration are given in Table 3.2

\begin{tabular}{|l|c|c|}
\hline & Before Calibration & After Calibration \\
\hline Mean $\mu$ & $4.9 \pm 0.2$ & $0.4 \pm 0.02$ \\
\hline Width $\sigma$ & $6.7 \pm 1.5$ & $4.2 \pm 0.3$ \\
\hline
\end{tabular}

Table 3.2: The means and widths of the $\Delta t_{0}$ distributions before and after calibration constants were applied

To estimate the timing resolution of a single hit in the shield, the error in the muon vertex time and the resolution of the projection back to the shield must be estimated. The timing resolution on a single detector hit, discussed in the previous section, was found to be $2.6 \mathrm{~ns}$. However, the vertex time is calculated by averaging over five planes, so the estimated error in the vertex time is $1.3 \mathrm{~ns}$. The error in the projection was estimated using Monte Carlo muons and found to be $1.2 \mathrm{~cm}$, which is equivalent to a time resolution of the order of $10 \mathrm{ps}$. The timing resolution for a single hit in the shield is therefore $4.0 \mathrm{~ns}$. 


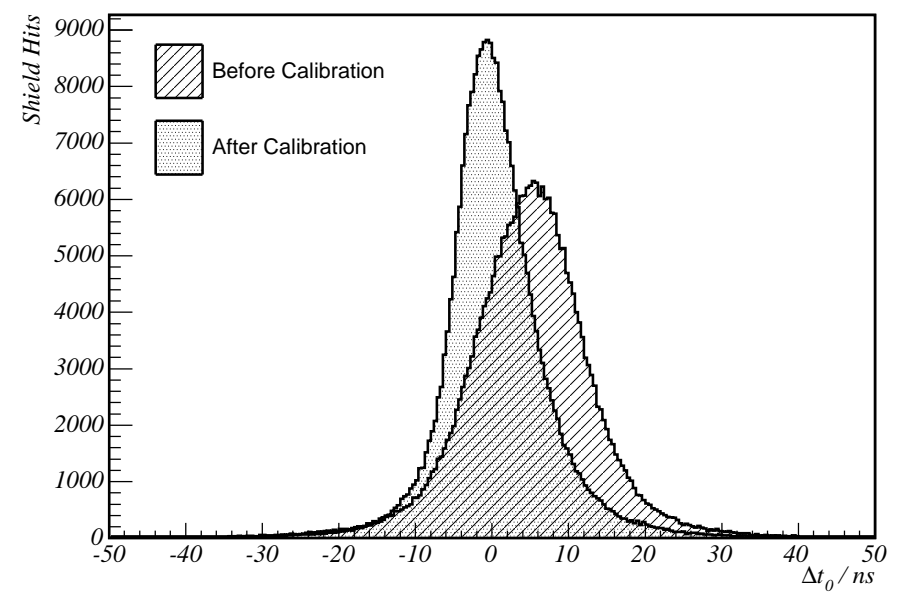

Figure 3.8: The veto shield timing resolution before and after the application of the timing calibration constants. The width of the timing resolution after calibration is $4.2 \mathrm{~ns}$

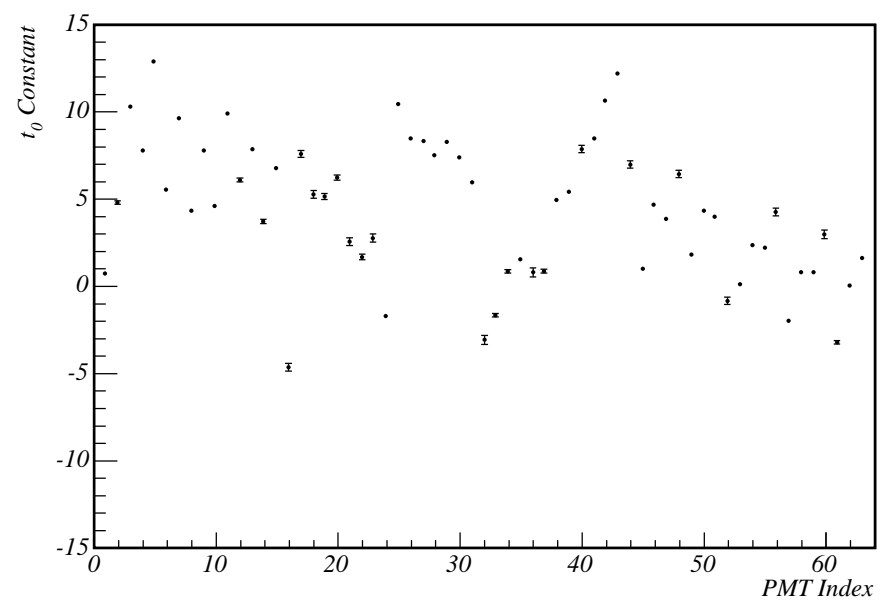

Figure 3.9: The timing calibration constants for the MINOS veto shield. Each point is the mean of the best fit Gaussian for the $\Delta t_{0}$ s for a PMT. The PMT index corresponds to a single PMT in the shield. 


\subsection{Summary}

The MINOS Far Detector construction was completed in mid July 2003. Veto shield construction was completed early August 2003. Since that time the detector has been taking cosmic muon data.

At the time of writing, the complete energy calibration of the Far Detector was unavailable. A PMT gain calibration without linearity or drift corrections was available, providing conversion from ADC counts to photoelectrons [50].

A single timing calibration was performed in January 2003 [51]. No further updates were made to this calibration at the time of writing. Ultimately the timing calibration will be repeated every two months. 


\section{Chapter 4}

\section{The MINOS Data Acquisition System}

Despite the difference in detector topology, number of channels and front-end electronics, all three MINOS detectors have essentially the same Data Acquisition System (DAQ).

The MINOS DAQ [52] has a modular design making it easily expandable. The use of commercially available hardware means that the system modules are robust, cheap and easy to replace. The extensive use of software throughout the system provides a high degree of flexibility. For example, system configurations such as trigger algorithms can be modified with small software changes. The remote location and high cost of staffing of the Soudan mine means that the DAQ must be a stable system requiring minimal operator intervention.

At the time of writing, the MINOS DAQ software consisted of approximately three hundred thousand lines of $\mathrm{C}$ and $\mathrm{C}++$ code to which the author made a central contribution including the Run Control server software, the Data Collection Processor and a number of support libraries such as robust network communication libraries and user messaging.

\subsection{Far Detector Data Acquisition System Operation}

Figure 4.1 shows a schematic overview of the DAQ system. Data from the front-end electronics (discussed in Section 3.2) is stored in the front-end buffers on custom made VME (Versa Module Europa) boards termed VA Readout Controllers or VARCs. Each VARC buffers the output of up to 36 photomultiplier tubes. There are 3 VARCs 
per VME DAQ crate and a total of sixteen DAQ crates. Each crate is read out under the supervision of the DAQ Read Out Processor (ROP).

The Read Out Processor consists of a CES RIO3 single board computer running the Wind River Systems VxWorks 5.4 real-time operating system. The ROPs run in disk-less mode, loading the operating system over the network at boot time.

The ROPs read data from alternating buffers allowing digitisation to continue during readout, incurring no dead-time. Read out is synchronised across all frontend crates by the Timing system Central Unit (TCU). The TCU fans out control signals to VME Timing Receiver Cards (TRCs) in each crate. The TRCs generate VME interrupts at a programmable rate, currently set to $40 \mathrm{~Hz}$. Upon receipt of a VME interrupt the ROP swaps front-end buffers and transfers the data into memory. Each data transfer is referred to as a time-block.

The ROP assembles the time-blocks over a programmable time period, currently one second, into timeframes. It adds a header containing crate timing information and timeframe number and appends some monitoring statistics. The last time block from each timeframe is repeated at the head of each new timeframe. This removes trigger inefficiencies due to physical events occurring near a timeframe boundary which could be split between two timeframes. Timeframes are then buffered in the ROP until requested for processing.

Up to four ROPs are daisy-chained together into one branch using a differential PCI Vertical InterConnect system (PVIC). Each branch is connected to a Branch Readout Processor (BRP) via an optical PVIC connection. PVIC is a multi-node PCI-PCI interconnect, allowing one node direct access to another node's memory. It provides a high speed data transfer mechanism without the CPU over head of serialisation methods.

The BRPs are responsible for assembling data from all the ROPs on their branch and transferring it to the trigger farm for processing. Each BRP is an Intel Pentium based PC running the Fermilab release of Redhat Linux. One BRP acts as a master, co-ordinating the readout of the others. The master BRP instructs all the BRPs to request the next timeframe from the memory of the ROPs. Once this has completed, it selects a Trigger Processor (TP) from the trigger farm based on a selectable least-loaded or round robin algorithm. The master BRP then instructs each $\mathrm{BRP}$ to transfer the timeframe to the TP via direct memory access over the output PVIC branch. By passing the appropriate target address to each BRP in turn, the timeframe for the whole detector can be assembled continuously in the TP memory. 


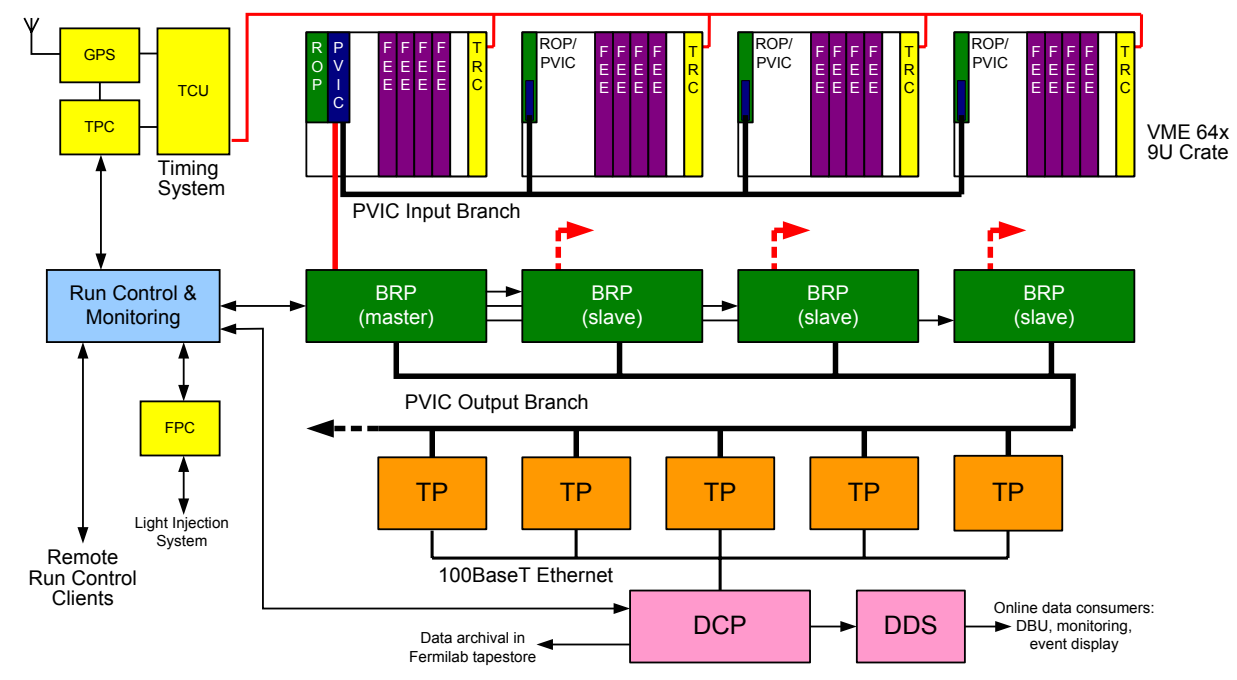

Figure 4.1: The architecture of the MINOS Far Detector DAQ system. For clarity only one of four PVIC input branches are shown. The system is essentially the same for both MINOS detectors apart from the timing system and front-end electronics.

\subsubsection{The Trigger Farm}

Upon receipt of a timeframe the TP performs a number of tasks. First, it timeorders the data on a crate level. It then performs data integrity checks ensuring the timeframes from all ROPs are correctly structured. Any calibration data is processed at this point and the results placed into the data output stream.

The TPs primary task is to look for events of physics interest. The TP divides data up into sections separated by at least 100 clock ticks (156 ns) with no hits, creating candidate events. The candidate events are then passed to a set of algorithms which look for clusters of detector activity. If the candidate event passes one or more of the algorithms, it is passed to the output stream. The actual algorithms used are configurable and an interface is provided to develop new ones. The algorithm used for the data presented in the following chapters requires that any four out of five contiguous planes in the detector have hits. A candidate event that passes one or more of the triggers is referred to as a snarl.

To investigate the efficiency of the 4-out-of-5 trigger algorithm for atmospheric neutrinos, the triggers were applied to a Monte Carlo sample of $\nu_{\mu}$ charged current events. The VARC 2-out-of-36 (discussed in the previous chapter) trigger was simulated and then the 4-out-of-5 plane trigger was applied. Figure 4.2 shows the DAQ 
trigger efficiency as a function of neutrino energy. The trigger efficiency is driven by the number of planes crossed by the event, 4 planes approximately corresponds to the range of a $200 \mathrm{MeV}$ muon at normal incidence to the planes.

Once processing in the trigger farm is complete, the physics event and monitoring statistics are moved to the output buffer. The $30 \mu \mathrm{s}$ of detector activity prior to the physics event is also written out. These data are called the pre-trigger window. The pre-trigger window can be used to flag channels that are dead due to detector noise or light-injection.

The output rate from the TP is typically two orders of magnitude lower than the input rate and can be transferred over TCP/IP to the Data Collection Process. The data rates for the Far Detector after DAQ triggers is shown in Table 4.1.

\begin{tabular}{|c|c|c|c|c|}
\hline & $\begin{array}{c}\text { Event Rate } \\
/ \mathrm{Hz}\end{array}$ & Events year & $\begin{array}{c}\text { Raw event } \\
\text { size }(\mathrm{kB})\end{array}$ & $\begin{array}{c}\text { Data Volume } \\
\left(\mathrm{GB} \mathrm{year}^{-1}\right)\end{array}$ \\
\hline Cosmic Ray Muons & 0.55 & $1.65 \times 10^{7}$ & 1.1 & 18 \\
\hline Noise & 6.5 & $1.95 \times 10^{8}$ & 0.2 & 39 \\
\hline Monitoring/Calibration & & & & 312 \\
\hline
\end{tabular}

Table 4.1: The event and raw data rates for the Far Detector after the DAQ trigger.

\subsubsection{Data Collection Process}

The Data Collection Process (DCP) collects processed timeframes from the trigger farm and performs a number of tasks:

- The time-ordering of data: In general, timeframe processing in the trigger farm does not finish in time order. The DCP buffers timeframes and time orders them before further processing.

- Data integrity checks: Checksum words in the data stream are tested upon receipt of a timeframe to flag corrupted data. If data corruption is detected, data-taking is stopped and the offending timeframe written to a debugging file.

- Removal of timeframe overlaps: To remove trigger inefficiencies at timeframe boundaries, the ROPs copy a programmable length of data from the end of one timeframe into the start of the next. For current DAQ settings and event rates, this leads to a $0.16 \%$ probability that a cosmic ray muon event can be found in two adjacent timeframes. The DCP examines adjacent timeframes and repeated events are removed before archival to file. 

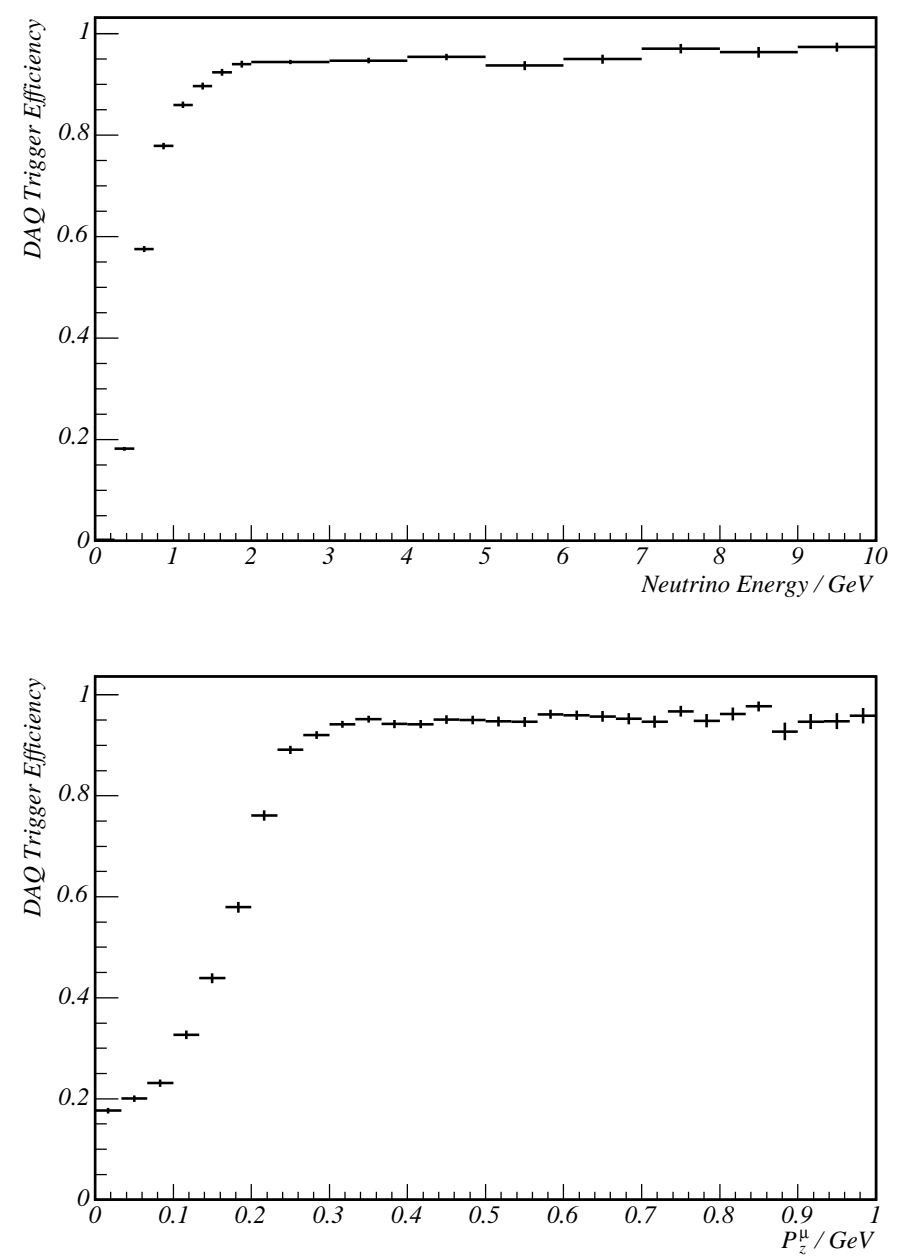

Figure 4.2: The DAQ Trigger efficiency for atmospheric $\nu_{\mu} \mathrm{CC}$ events as a function of (top) neutrino energy (bottom) the $Z$ component of muon momentum.

- Write the data to file: Data can be written in either ROOT [53] format or written in the native MINOS DAQ format. The output files are supplied to the MINOS Data Distribution System which distributes data to the quasi online processes such as online monitoring and event displays. Once closed, a file is archived to Fermilab mass storage system via File Transfer Protocol (FTP).

- Monitor data flow rate and DAQ disk usage: By examining the data the DCP reports the current dynode trigger rates in each crate to the operator. It also supplies information on disk usage. 
- Run configuration archival: The DCP archives the current DAQ and run configuration to file with the data, allowing easy reconstruction of exact DAQ conditions in data analysis.

The responsibilities of the DCP are divided into two groups and each group is handled by a different process. The two processes transfer data between themselves via a buffer system residing in shared memory. The first process handles requests for data transfer from the trigger farm. Upon a successful request the timeframe is transferred over a TCP/IP socket to the DCP which writes it to a free buffer and flags it for processing. The DCP has a finite number of buffers and must process timeframes in time order. It will therefore only grant a request for the transfer of timeframe $\mathrm{N}$ if the number of free buffers is less than the difference between $\mathrm{N}$ and the current timeframe being processed. This ensures that the DCP will always have room for the next timeframe required for processing. The first DCP processes also handles Run Control communication, data integrity monitoring and statistics reporting.

A second DCP process takes the current and next to current timeframe from the buffer system, removes any event overlaps near the timeframe boundary and writes the data to file in time order. The output to disk is the slowest part of the DAQ. It is therefore separated out into its own process in order to ensure responsiveness to trigger farm requests.

The mean data rate through the DCP under normal data-taking conditions is $15 \mathrm{kBs}^{-1}$. However, the maximum continuous data through-put obtained during tests is $8 \mathrm{MBs}^{-1}$, on a $700 \mathrm{MHz}$ Intel PIII with $128 \mathrm{MB}$ memory. However, with file compression set to optimal values the maximum continuous rate supported is approximately $4 \mathrm{MBs}^{-1}$.

\subsection{Run Control}

The MINOS DAQ system is monitored and co-ordinated by the Run Control system. Run Control also provides the contact point between the operator and the DAQ. It allows the operator to control and run diagnostics on the DAQ while providing feedback in real time. The system requirements are listed in full in [54] and are broadly summarised below.

1. Run Control must be able to determine and display to the operator the state of all the DAQ components and the rate of data-flow through them. 
2. Run Control must allow the operator to change run conditions and detector configuration via a user friendly interface and communicate these changes to all parts of the DAQ.

3. Run Control must co-ordinate the changes in run state between all DAQ components. Run conditions must be static during a run and each run must be identified by a unique number.

4. Run Control must allow multiple users to observe the current DAQ status, but only one user should have control of the DAQ at any one time. The users may be distributed throughout the world and a well defined control transfer protocol must be provided.

5. Run Control should be able to run the detector under normal conditions without operator intervention, taking calibration and diagnostic runs with the required periodicity.

The Run Control system was designed during summer 2001 and implemented and deployed over the following year. It has been installed at all three detector locations and is currently in version 3.2.1.

\subsubsection{Design and Implementation}

\section{Overall Structure}

Run Control is based on the client-server model. A central server runs at each detector location and each location is a totally independent system. Light-weight clients can then connect to the server via the internet. Clients do not contain any control logic but purely provide a user interface. The clients are under the control of client management software in the central server. It is this software that decides which client can issue commands to the system and handles the transfer of control between them. The client in current use is a ROOT [53] based Graphical User Interface implementation developed at Cambridge [55]. Run Control also has a passive web and Wireless Application Protocol (WAP) interface for use on mobile phones. No commands can be issued to the DAQ from the passive interfaces, they purely display the current DAQ state and the data transfer rates.

The Run Control server connects to all DAQ processes over TCP/IP sockets using a protocol designed specifically for this task. All configuration, commands, state reports and monitoring data are passed over the socket connections. Run Control is of object-oriented design and is implemented in $\mathrm{C} / \mathrm{C}++$. 


\begin{tabular}{|c|l|}
\hline IDLE & $\begin{array}{l}\text { The node is not participating in data taking. This } \\
\text { the default state of all nodes at start-up. A node } \\
\text { stays in this state until instructed to prepare for } \\
\text { data taking by Run Control. }\end{array}$ \\
\hline PREPARED & $\begin{array}{l}\text { The node has successfully configured itself for data } \\
\text { taking according to the parameters provided by } \\
\text { Run Control. Data-taking does not start until in- } \\
\text { structed to do so by Run Control. }\end{array}$ \\
\hline RUNNING & $\begin{array}{l}\text { The node is currently involved in data taking and } \\
\text { will continue to be until instructed to stop by Run } \\
\text { Control or an error occurs. }\end{array}$ \\
\hline ERROR & $\begin{array}{l}\text { The node is no longer able to continue with data } \\
\text { taking or a state transition has failed. }\end{array}$ \\
\hline
\end{tabular}

Table 4.2: The primary DAQ states.

\section{Run States}

The DAQ consists of several logical nodes; DCP, Trigger Farm Processor (TFP), MBRP, light injection calibration system (FPC), the ROPs, the BRPs, the Timing System (TPC) and some quasi-online processes which are not strictly part of the DAQ but are still monitored by Run Control, for example the Detector Control System (DCS). Each node is responsible for maintaining its own state, which in turn may depend on the state of one or more dependants, for example, the MBRP derives its state from the state of the BRPs, which in turn derive their state from the states of the ROPs that they manage. Each node must conform to the state model which describes the various run states and the allowed transitions between them.

The Run Model used in the MINOS DAQ is described in detail in [56]. Figure 4.3 shows the finite state machine that each node must follow. There are a well defined set of primary states and transitions between them. The primary states are defined in Table 4.2. A node can move through a state transition under the command of Run Control, the exception being the ERROR state which the node enters when it is no longer able to continue with data taking or complete a state transition.

Run Control maintains a DAQ Global state, which is dependant on the state of the nodes and conforms to the same state machine described above. The list of nodes from which the Global state derives can be dynamically edited by the operator for debugging purposes. Some node's state transitions take a finite length of time and require synchronisation with other nodes. This logic is contained within the Global 


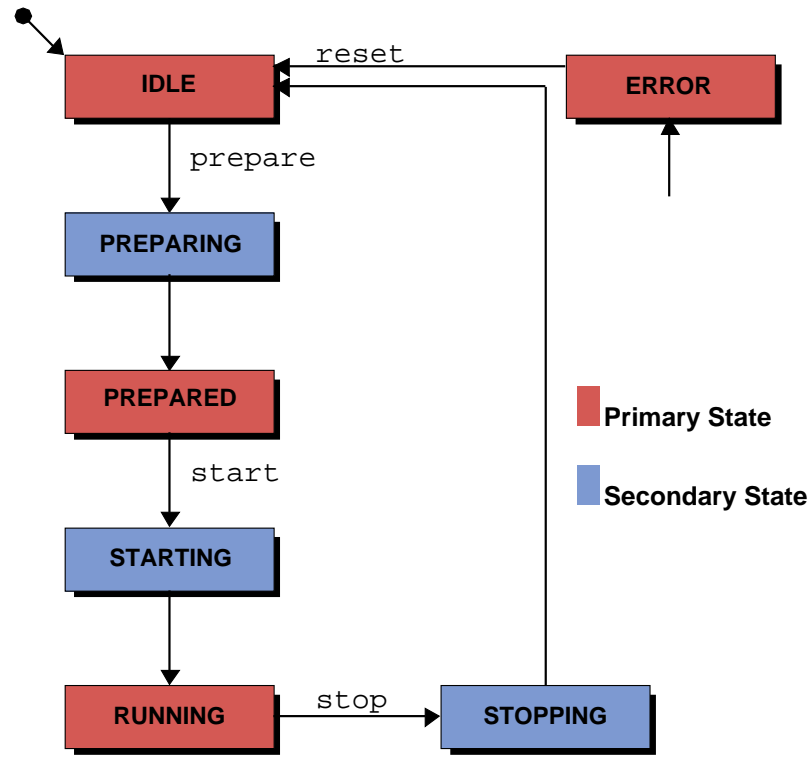

Figure 4.3: The finite state machine for the DAQ run model. Note that the implicit abort command which is valid from any state is not shown. Also the implicit transitions from any state to the ERROR state is not shown.

state. For example, to start a run, Run Control issues a single command to the Global state, which then starts nodes in the correct sequence and distributes required configuration information. If any dependant node is in the ERROR state, the Global state will move to ERROR from which the error recovery logic can be executed with the reset command. If the dependant nodes are in a mixture of non-ERROR states and Global is not in a state transition, Global moves to a special state UNKNOWN and user intervention is required to bring the DAQ back to a well defined state. Run Control allows the issuing of commands directly to nodes by-passing Global to recover from UNKNOWN states. The Global state machine was implemented using the State and Command Design Patterns [57].

\section{System Messaging}

It is a design requirement that DAQ processes should log all activity to a central location, and that these logs should be viewable by the operator in real time. The logging should not interfere with data-taking. All DAQ processes use the messaging facility not just those connected directly to the Run Control server, therefore using the Run Control socket protocol was not a viable solution. An interface similar to 
that of the UNIX syslog process was required. A messaging library, MsgLogLib, was developed for the LINUX platform. MsgLogLib sends messages directly to the Run Control server via a User Datagram Protocol (UDP) connection. UDP is a connectionless protocol and is therefore not dependant on the destination socket being open. Processes using MsgLogLib are therefore guaranteed not to block, regardless of network status. The message protocol flags each message with Process ID, MINOS DAQ node ID, Host IP and a message sequence number allowing the receiving end to tell if a message has been dropped. The messages are read by the Run Control Server, displayed on the user interface and archived to file.

\section{Process Management}

Run Control provides the user with a single interface for processes management. The status of every DAQ process can be queried and re-started. Processes management is provided by a scripting interface to the Run Control server. Expert users can provide scripts for Run Control to execute on the DAQ system, the output of which is presented to the user on the client interface. The scripting service is also used to provide a unified interface for general DAQ maintenance tasks that must be carried out during shifts. The scripting interface removes the need for anyone other than expert users to log on to DAQ systems. Instead, all DAQ related maintenance can be executed directly from the GUI.

\section{Run Sequencer}

Run Control provides an automated running service, the Run Sequencer. A sequence of runs can be defined by the Run Control user by passing the Run Sequencer a XML (Extensible Markup Language [58]) file. Each run in the sequence has a run duration and a configuration string. When the user loads a sequence XML file into the Run Sequencer, it takes control of the Run Control state machine and will configure and take the defined runs automatically. It can either repeat the sequence indefinitely or stop the sequence after a configurable number of iterations. The XML file configuration is extendable via a custom written plug-in system. Users can provide a dynamically loadable library that can provide arbitrarily complex sequence and run configurations. The advantage of the plug-in system is that the libraries can be loaded when required. Users can, therefore, develop complex run sequences without stopping the Run Control process. 


\subsection{Summary}

The MINOS DAQ is designed to be cheap, robust and expandable. The use of off-the-shelf hardware throughout has allowed a high redundancy to be built into the system. Run Control has been designed and implemented to be highly flexible and expandable. The heavy use of dynamically loadable libraries and the objectorientated design means expansion of the current system or even addition of new sub detectors would require only trivial changes to the Run Control server. All routine DAQ tasks can be performed directly from a single client GUI screen, eliminating the need to allow login access to shift workers.

The MINOS DAQ has been deployed at five different locations over the past three years including the MINOS Far Detector, Near Detector, Calibration Detector and two test locations. 


\section{Chapter 5}

\section{Monte Carlo Simulation}

The analysis presented in the following chapters was developed, tuned and interpreted using Monte Carlo simulations of signal and background events. It is therefore important to understand the Monte Carlo implementation, in particular the limitations that might give rise to systematic errors in the final results obtained.

\subsection{Monte Carlo}

\subsubsection{Atmospheric Neutrino Monte Carlo Flux}

There are several models of atmospheric neutrino flux available (for example [59, 60, 61, 62]), although they all share some basic ingredients:

- Primary Cosmic Muon Flux. The flux of cosmic particles, predominately protons, striking the upper atmosphere.

- Geomagnetic Effects. The effect of the Earth's magnetic field on the primary and secondary cosmic ray particles.

- Hadronic Interactions. The propagation and interactions of intermediate secondary particles.

- 3 Dimensional Effects. Until recently, the atmospheric neutrino flux calculations were all one dimensional, where all particles produced were assumed to be along the direction of the initial primary cosmic ray. However, the effect of the opening angle between the neutrino and is parent particle has been included by Battistoni et al [60, Honda et al [62] and more recently by Barr et al [61]. The 
calculations by Barr et al have also included the effect of the Earth's magnetic field on the propagation of the air showers.

These features are discussed in more detail below. Particular attention is paid to the Battistoni et al calculation used for the analysis presented in this thesis.

\section{Primary Cosmic Ray Flux}

The origin of the primary cosmic rays is still not understood. However, it is believed that the origin of the flux is within the Galaxy [63]. The cosmic rays are made up of approximately $90 \%$ protons, $9 \%$ alpha particles and the rest are heavier nuclei [64]. The cosmic ray flux has been well measured up to $100 \mathrm{GeV}$ per nucleon in balloon-borne magnetic spectrometers, for example [65, 66, 67, 68, 69] and the AMS space shuttle flight [70]. Higher energies have been probed using balloon-borne calorimenters [71, 72]. However, the spectrum at these higher energies tends to be less precisely measured because the flux is much lower and the calorimeters have poorer energy resolution than the spectrometers. Very high energy flux measurements are not possible with balloon experiments because the low flux requires large detectors exposed for a long period of time. Ground based experiments, such as AGASA [73] and Auger [74], detect the large air showers initiated by high energy incident cosmic rays. However, these experiments suffer from large statistical errors due to the low flux at high energies and larger systematic errors due to the difficultly of inferring the primary flux from the air showers detected by the experiment.

The observed spectrum is summarised in Figure 5.1. Cosmic rays are well described by a power law of the form;

$$
\frac{\mathrm{dN}}{\mathrm{dE}} \propto E^{-\gamma}
$$

where $\gamma=2.7$ for energies less than $10^{6} \mathrm{GeV}$, a region known as below the knee, and $\gamma=3.0$ above the knee.

The sun emits a magnetised plasma called the solar wind. The period between successive solar maxima is approximately 11 years [75]. To reach the Earth's atmosphere, the cosmic rays have to diffuse through the outward flow of the solar wind, the effect of which is that solar activity modulates the lower energy region of the cosmic ray flux. For the Soudan site the effect is estimated to be approximately $20 \%$ for $10 \mathrm{GeV}$ protons and nearly a factor of 2 for protons below $1 \mathrm{GeV}$. The last solar maximum occurred in 2000. The flux used in this thesis was calculated at solar max- 

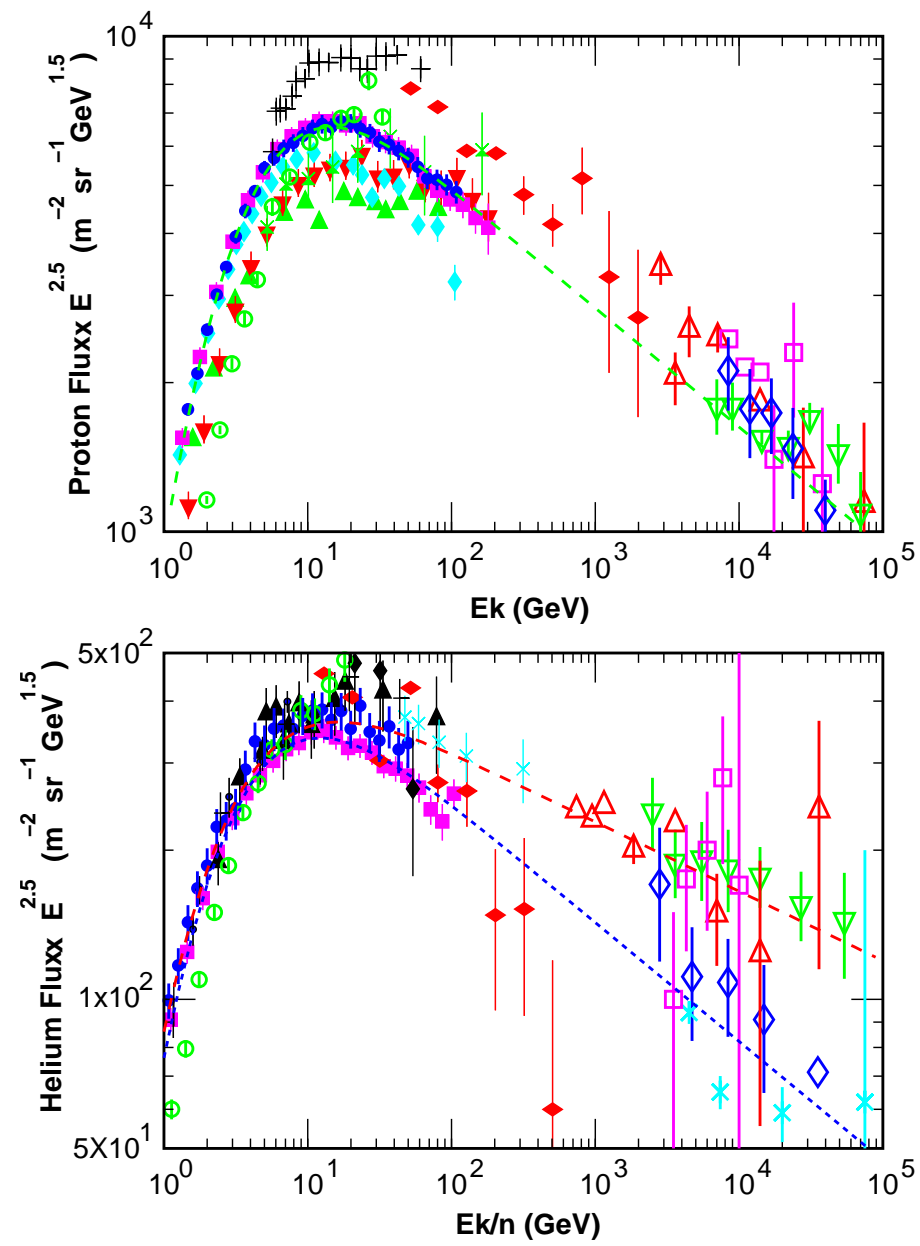

Figure 5.1: The observed flux of primary cosmic ray protons and helium nuclei. The dashed lines show a commonly used parameterisation which include different data sets. The data points are Webber (crosses), LEAP (upwards triangles), MASS1 (open circles), CAPRICE (vertical diamonds), IMAX (downward triangles), BESS98 (circles), AMS (squares), Ryan (horizontal diamonds), JACEE (downward open triangles), Ivanenko (upward open triangles), Kawamura (open squares) and Runjob (open diamonds). References can be found in [64]. 
imum and variations in solar activity over the period of data-taking were estimated to be of the order of $1 \%$.

\section{Geomagnetic Effects}

The positively charged primary cosmic rays interact with the Earth's magnetic field. Particles with low rigidity (defined as particle momentum over charge) are deflected before they strike the atmosphere and do not contribute to the observed neutrino and cosmic ray muon flux. The minimum rigidity required for a cosmic ray particle to strike the top of the atmosphere is called the 'cutoff rigidity' and is a function of location and direction of travel of the cosmic ray. Particles arriving from the East have a higher cutoff than particles arriving from the West due to the interaction of a positive particle with the Earth's magnetic field. Therefore, the cosmic ray flux from the West is greater than that from the East. However, due to the high magnetic latitude of the Soudan site, the east-west asymmetry is expected to be small.

In the $1 \mathrm{D}$ calculations, the produced neutrino was assumed to be collinear to the parent cosmic ray. In this case the cutoff can be calculated for every position on the Earth's atmosphere, because only one direction can contribute to the flux at the detector site. To calculate the minimum rigidity at each point, the time/charge reversible nature of the field equations is exploited. A test particle of negative charge is fired upwards from the top of the atmosphere. If the test particle can escape the Earth's magnetic field then the event is kept. However, the Battistoni flux is a semi-3D calculation, accounting for the opening angle between the neutrinos and their parent particles. Therefore, at each location at the top of the atmosphere, there are many incident angles that can produce a neutrino at the detector site. Once a neutrino is produced, the interaction chain that created it is rotated until the neutrino strikes the detector. The cut-off is then applied in the normal way using the relative position and trajectory of the parent cosmic ray.

\section{Hadronic Interactions}

The dominant source of uncertainty in the predicted neutrino flux is the hadronic interaction model, in particular the pion production model. These models are tuned to the accelerator data for protons on light nuclei. Most of these experiments were studies for accelerator design, hence the phase space explored does not fully cover the phase space of the meson production in the atmosphere. Figure 5.2 shows the regions of phase space for proton-air interactions that contribute to contained atmospheric 
neutrinos. Superimposed on Figure 5.2 are the regions of phase space covered by the data sets used for tuning the hadroproduction models. It is therefore necessary to extrapolate and interpolate into unmapped areas of phase space.

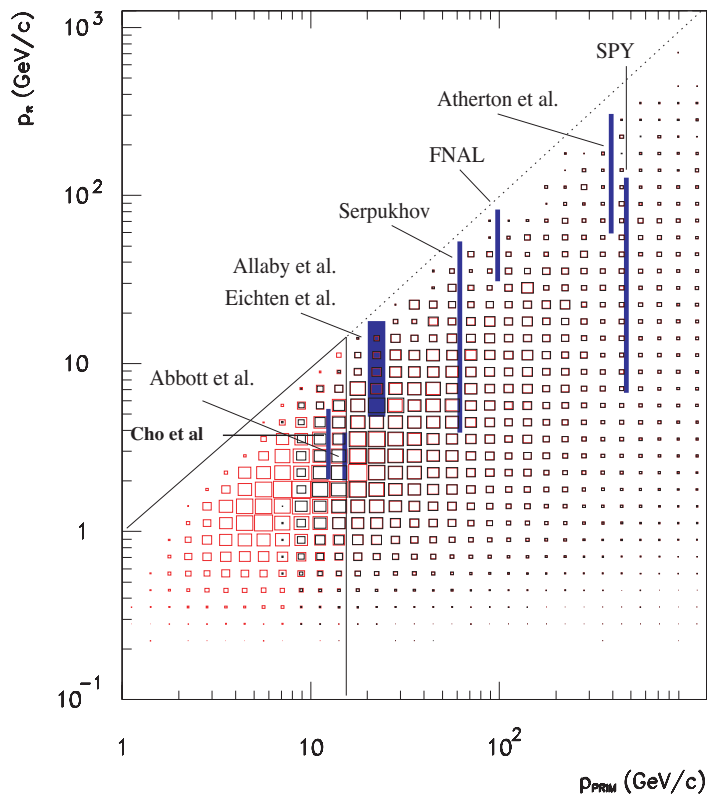

Figure 5.2: The weighted phase space distribution for atmospheric neutrinos at SuperKamiokande, calculated using the FLUKA model. The main data sets used for tuning the hadronic models are also shown. Figure taken from [64].

The Battistoni flux model uses the FLUKA cascade model, which shows $10 \%$ agreement with data for all measured regions of phase space. The Battistoni group estimate the contribution to the overall flux normalisation uncertainty from hadronic models to be approximately $15 \%$.

\subsubsection{General Features Of Atmospheric Fluxes}

The dominant process for neutrino production in the atmosphere is the leptonic decay of pions created in the hadronic showers of the primary cosmic ray flux,

$$
\begin{aligned}
& \pi^{ \pm} \rightarrow \mu^{ \pm}+\nu_{\mu}\left(\bar{\nu}_{\mu}\right) \\
& \mu^{ \pm} \rightarrow e^{ \pm}+\nu_{e}\left(\bar{\nu}_{e}\right)+\bar{\nu}_{\mu}\left(\nu_{\mu}\right) .
\end{aligned}
$$

The leptonic decays of kaons also produce neutrinos. However, kaons are not a significant source of neutrino production until neutrino energies of approximately 
$100 \mathrm{GeV}$. The predicted energy spectrum for $\nu_{\mu}$ at Soudan from the Battastoni flux calculation is shown in Figure 5.3 .

Assuming that all the muons decay, we can use Equation 5.1 to predict some general features of the neutrino flux in the absence of oscillations;

$$
\begin{aligned}
\frac{\nu_{e}+\bar{\nu}_{e}}{\nu_{\mu}+\bar{\nu}_{\mu}} & =1 / 2 \\
\nu_{\mu} / \bar{\nu}_{\mu} & =1
\end{aligned}
$$

As the primary energy increases, more muons reach the ground before decay. Thus for neutrino energies above a few $\mathrm{GeV}$, the charge ratio, $\nu_{\mu} / \bar{\nu}_{\mu}$ is expected to tend to the charge ratio of pions, approximately 1.2 [64].

The zenith angle dependence of neutrino flux is shown for four different energy ranges in Figure 5.4. The low energy sample shows a significant excess of downwardgoing neutrino events, reflecting the fact that the Soudan site has a low geomagnetic cutoff, so that more low energy primary cosmic rays reach the atmosphere above Soudan than the average for the rest of the world.

\subsubsection{Neutrino Cross-Sections}

Neutrinos interact with the nuclei in the detector via the charged current (CC) interaction to produce muons, Figure 5.5 .

The NEUGEN3 package [77] was used to model the neutrino interactions. NEUGEN simulates three distinct types of charged current interaction; quasi-elastic $(Q E)$, resonance production (res) and deep inelastic scattering $(D I S)$. The contribution to the total cross section from coherent pion production is not currently included in NEUGEN. The contribution to the total cross section from each of the simulated processes is shown in Figure 5.6. It should be noted that in the energy range of interest for contained events, $0.5-6 \mathrm{GeV}$, several production methods contribute to the total cross section.

The steeply falling neutrino flux combined with the dominance of the quasi-elastic process at low energies means that the majority $(60 \%)$ of contained events are quasielastic, i.e.

$$
\begin{gathered}
\nu_{\mu}+n \rightarrow \mu^{-}+p \\
\bar{\nu}_{\mu}+p \rightarrow \mu^{+}+n
\end{gathered}
$$

Figure 5.7 shows the contribution of the quasi-elastic events compared to data. The 

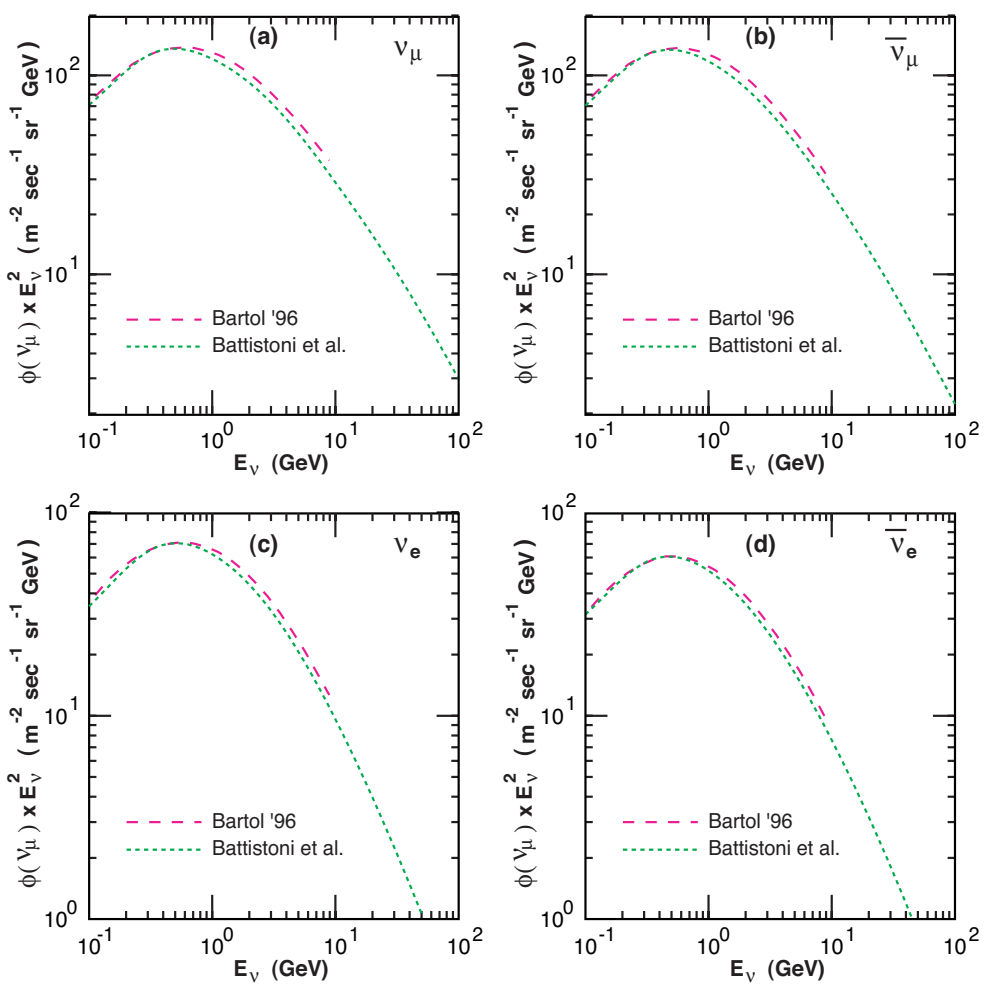

Figure 5.3: The flux of different neutrino types, (a) $\nu_{\mu}$, (b) $\bar{\nu}_{\mu}$, (c) $\nu_{e}$ and (d) $\bar{\nu}_{e}$, as a function of neutrino energy from the Battistoni et al calculations [60]. The Bartol'96 [59] which is a 1D calculation used by the Soudan 2 experiment [29], is shown for comparison.

low energy region, dominated by the quasi-elastic process, has not been extensively experimentally measured. The uncertainty in the contribution of the quasi-elastic process to the total cross section is estimated to be $20 \%$ [78].

Resonance production accounts for about $30 \%$ of the events selected in the analysis presented in this thesis. Resonance production at low energies such as those studied here is dominated by $\Delta(1232)$, although 17 different resonances are included in NEUGEN. The dominant final product of resonance production is a single pion,

$$
\nu_{\mu}+p \rightarrow \mu^{-}+p+\pi^{+} .
$$



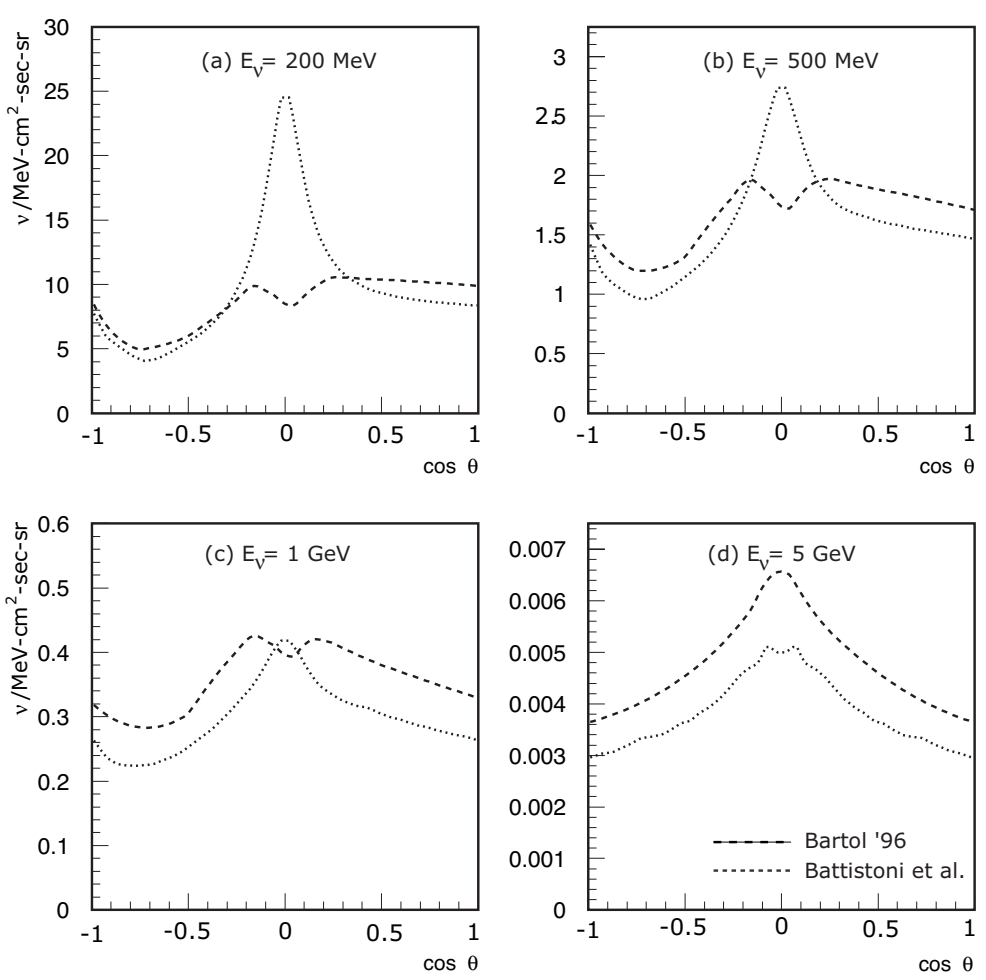

Figure 5.4: The cosine zenith angle dependance of the neutrino flux for four different energy bins. The Bartol'96 model which is a 1D calculation is also included for comparison. Figure taken from [76].

However, some resonances can result in multi-pion final states. Deep inelastic scattering mostly creates multi-pion final states.

The uncertainty in the total cross section is estimated to be $10 \%$ for both neutrinos and anti-neutrinos [78]. A summary of measurements for both $\nu_{\mu}$ and $\bar{\nu}_{\mu}$ charged current cross sections is shown in Figure 5.8 and the ratio of cross sections from NEUGEN is shown in Figure 5.9. The systematic uncertainty in ratio of neutrino to anti-neutrino cross sections is conservatively estimated to be $15 \%$ [8].

\subsubsection{Systematic Uncertainties on Number of Neutrino Interactions}

The systematic uncertainties in the neutrino flux are mostly attributed to uncertainties in the primary spectrum and the treatment of hadronic interactions in the atmosphere. However, there are additional minor uncertainties due to technical aspects of the simulation and the model of the atmosphere used. The estimated uncertainty in the fit to all current primary cosmic ray data below $100 \mathrm{GeV}$ is $7 \%$ [60]. The 


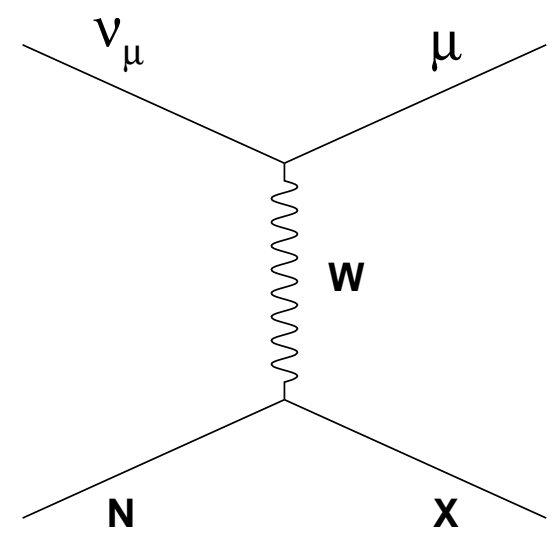

Figure 5.5: A schematic of the muon neutrino charged current interaction on a nucleus $\mathrm{N}$. X depends on the form of the interaction.

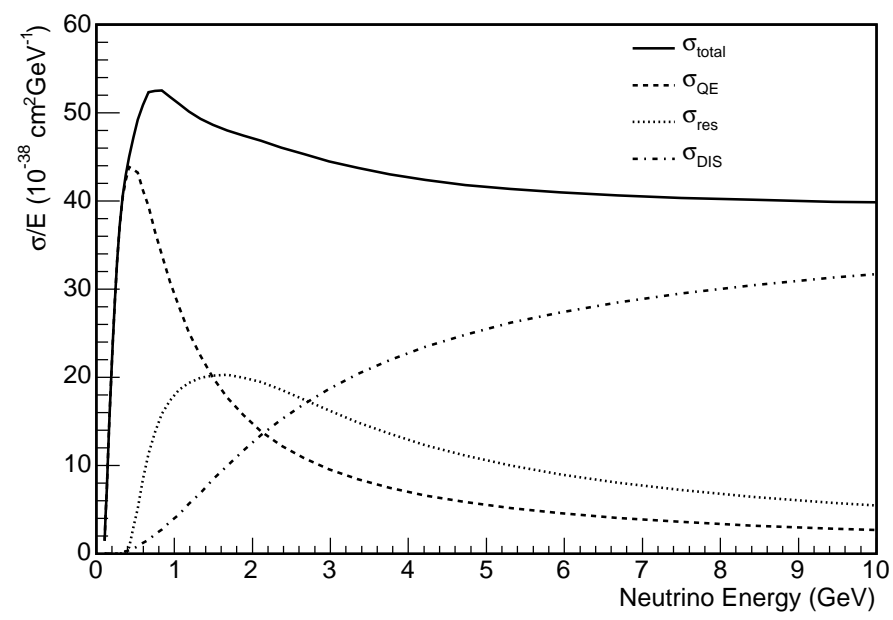

Figure 5.6: The contributions to the total cross section from the different simulated processes in NEUGEN3; quasi-elastic (QE), resonance production (res) and deep inelastic scattering (DIS).

estimated error on the hadronic production model is $15 \%$. The effect on the total flux due to density variations in the atmosphere is estimated to be less than $1 \%$. The effect of the geomagnetic field on the propagation of showers is estimated to carry an uncertainty of approximately $2 \%$. The overall uncertainty in the neutrino flux is therefore estimated to be approximately 17\%. An additional 10\% comes from the uncertainty in the total neutrino cross section. The overall systematic uncertainty in the number of neutrino interactions is therefore taken to be $20 \%$. 


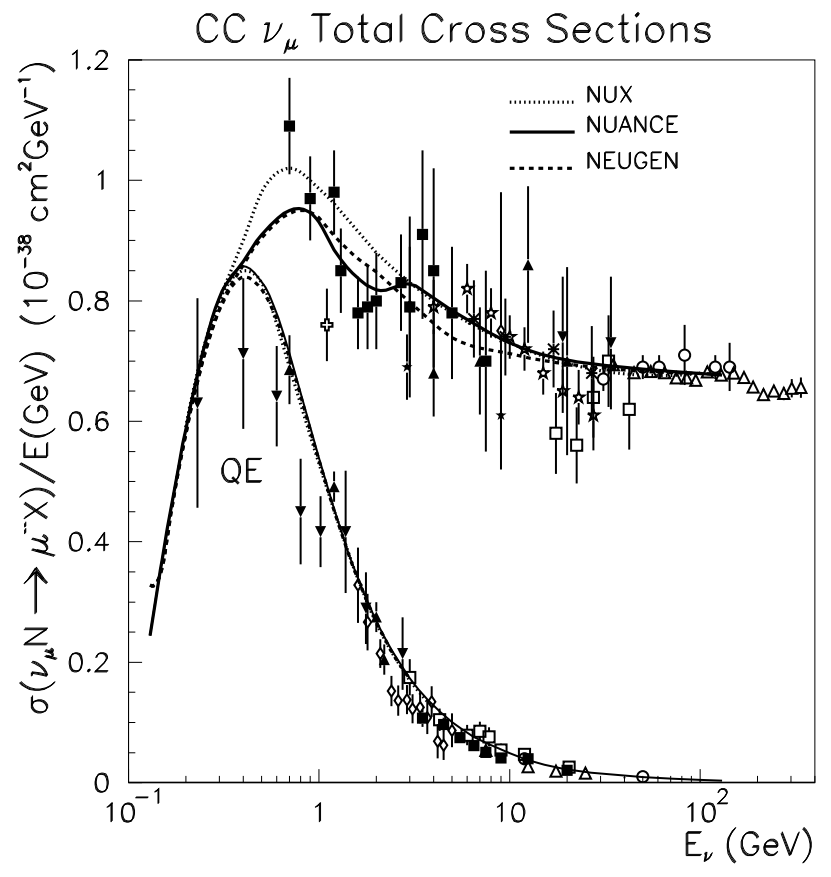

Figure 5.7: The total and quasi-elastic neutrino cross sections as a function of neutrino energy compared to data. The predictions from two other neutrino event generators (NUX [79] and NUANCE [80]) are also shown. Plot taken from [81].

\subsubsection{Cosmic Ray Muon Monte Carlo Flux}

The cosmic ray muons from high energy pion and kaon decays are the main source of background in the analysis presented in this thesis. The cosmic ray muon Monte Carlo sample was generated by propagating a parameterisation of muon flux at the surface [82] through a map of the rock overburden generated by the Soudan 2 callaboration [83]. The overall normalisation on the cosmic ray muon Monte Carlo was determined by comparing the number of through-going muons selected in data and Monte Carlo. A second estimate was made using the cosmic ray muon events that 


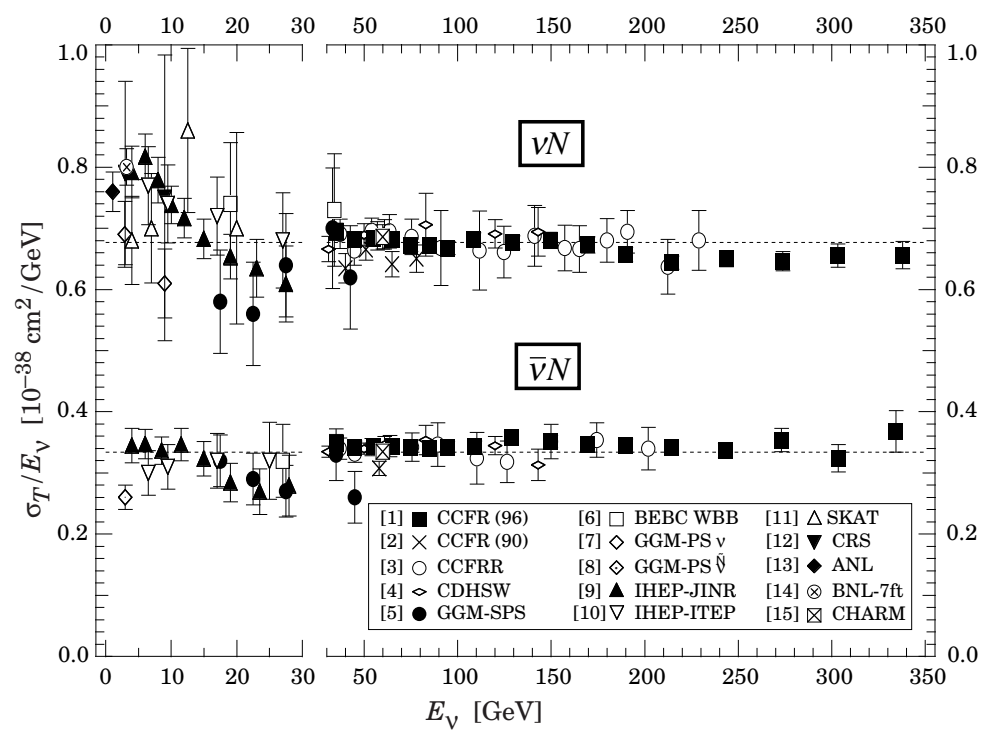

Figure 5.8: The $\nu_{\mu}$ and $\bar{\nu}_{\mu}$ charged current cross sections as a function of energy (taken from [6]). The error bars indicate both statistical and systematic errors and the dotted lines are the mean values.

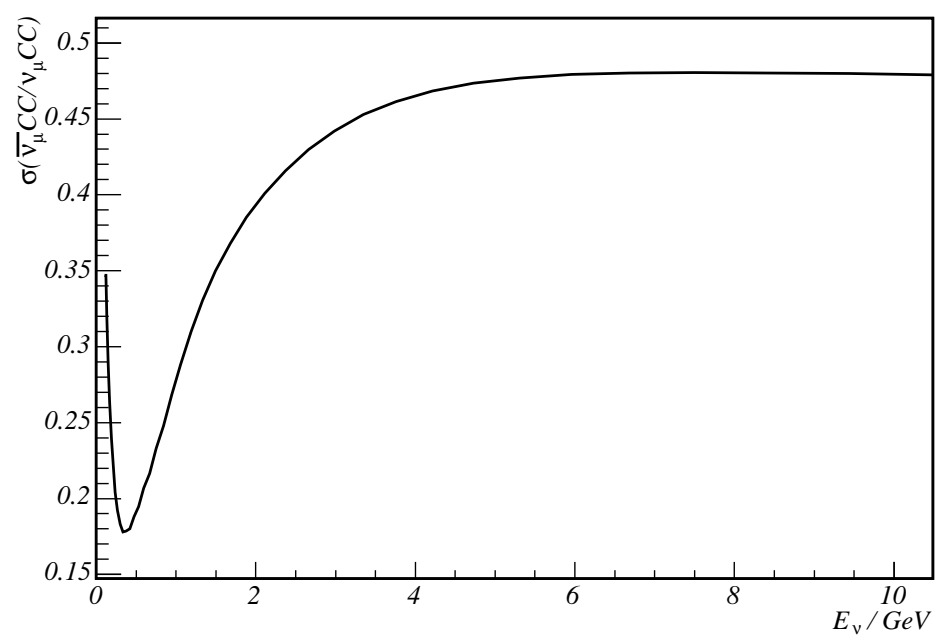

Figure 5.9: Ratio of cross sections $\bar{\nu}_{\mu} / \nu_{\mu}$ as a function of energy as calculated by NEUGEN3 
stop in the detector. The difference between these two values $(15 \%)$ was taken to be the systematic error on the normalisation and is attributed to inaccuracies in the muon momentum spectrum model used in the simulation.

\subsubsection{Particle Tracking and Detector Simulation}

The propagation of particles through the detector is performed using the GEANT3 package [84. The simulation of photon capture in the wavelength shifting fibre and the propagation of photons to the PMT face was performed by the PhotonTransport package [85]. The PMT and electronics response was simulated by the DetSim package 85$]$. Both PhotonTransport and DetSim are available in release 1.5 of Minossoft.

The Monte Carlo output was reconstructed using the same software used for data reconstruction. Information about the simulated neutrino and all the particles produced in the interaction is retained. This information is referred to as the 'truth' information.

\subsubsection{Monte Carlo Production}

Two cosmic ray muon samples were generated, the Full Spectrum and the Reduced Spectrum. The Full Spectrum sample consisted of 2.5 million events with a full energy spectrum, corresponding to 60 days live-time. The Reduced Spectrum sample consisted of 2.5 million events with $E_{\mu}<20 \mathrm{GeV}$, corresponding to 1 year of livetime, approximately twice the exposure of the data used in this thesis. This is the energy range occupied by the cosmic ray muon background in this analysis.

A sample of 1.5 million atmospheric neutrinos (equivalent to 520 kton-years or 96 years of data) was generated using the Battistoni flux and the GCALOR package [86]. A calibration detector (CalDet) was placed in a proton and pion beam with energies in the range $0.2-3.6 \mathrm{GeV}$. The GCALOR package was found to give the best description of the detector response to single pions and protons. Small atmospheric neutrino samples were generated using the FLUKA 87] and SLAC-GHEISHA [88] hadronic interaction models for the purposes of comparison with GCALOR.

\subsection{Data and Monte Carlo Comparison}

To study the accuracy with which the Monte Carlo simulation describes the data, a sample of cosmic ray muons that stop within the fiducial volume was selected. These 
events have momenta in a similar range to the contained muons produced in $\nu_{\mu} \mathrm{CC}$ events, making them an ideal test beam.

The Reduced Spectrum cosmic ray muon Monte Carlo was compared to the data. The contribution to stopping cosmic ray muons from energies not included in the Reduced Spectrum sample was less than $1 \%$.

Both the data and Monte Carlo were demultiplexed using the AltDeMux package [89] and track reconstruction was performed using the AtNuReco package [90]. A set of 11,000 stopping muons was selected from data by requiring that the reconstructed track deposited energy in 8 or more planes and just one track end be contained within the fiducial volume ${ }^{1}$. The track was also required to have at least six track-like planes, where a track-like plane is defined to have $50 \%$ or more of the charge in the plane within \pm 1 strip of the reconstructed track.

\subsubsection{Event Timing}

Once a track has been found, the direction of muon propagation is determined from the times of the hits along the track 2 . The flat overburden of Soudan mine means that there are no cosmic ray muons from below the horizontal, i.e. all cosmic ray muons are downward-going. The direction reconstruction efficiency can be determined for both the data and the Monte Carlo sample by investigating how many cosmic ray muons entering the detector from above and stop within the fiducial volume are reconstructed as upward going. The direction reconstruction efficiency for both data and Monte Carlo for cosmic ray muon events which traverse more than 8 planes is shown in Figure 5.10. It should be noted that there is a maximum of $4 \%$ disagreement between Monte Carlo and the data. The most probable cause for this discrepancy is imperfect timing calibration of the data. The calibration was last determined in January 2003 [51], since then various parts of the electronics have been replaced. However, the calibration was not recalculated for the new components. Figure 5.11 shows the timing resolution bias for each plane in the detector, regions of systematic offset can be seen where components have been replaced.

\subsubsection{Strip Response and Energy Deposition}

The gains of the photomultiplier tubes have been calibrated using the light-injection system, giving an ADC-count to photoelectron conversion for each tube. However,

\footnotetext{
${ }^{1}$ The definition of the fiducial volume is discussed in Section 6.3 .2

${ }^{2}$ The details of the track direction reconstruction are described in Section 7.1.1
} 


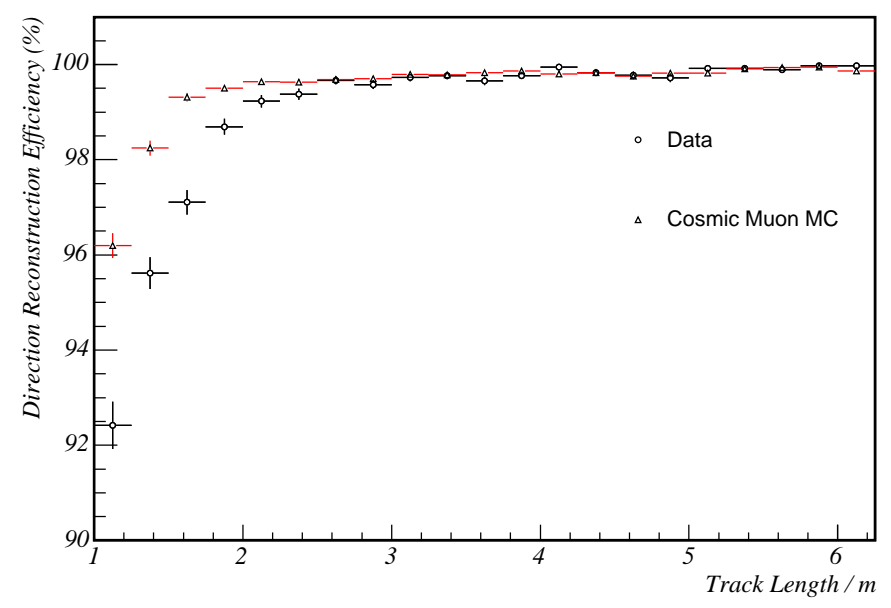

Figure 5.10: The probability of correctly identifying the direction of a downwardgoing cosmic ray muon track that stops in the detector as a function of track length.

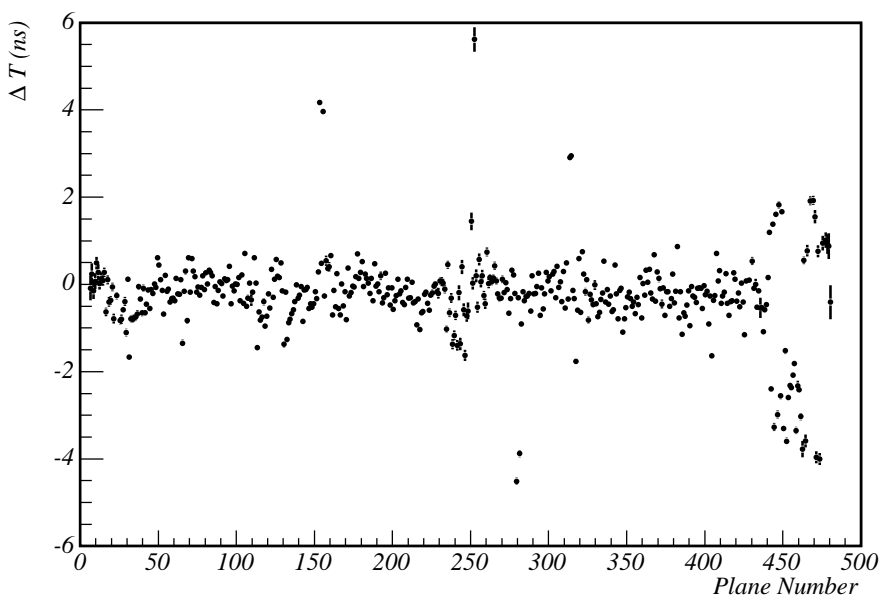

Figure 5.11: The mean difference $(\Delta T)$ between expected time of a digit on the East side minus the expected time on the West side, $\left(t_{E}-t_{W}\right)_{\text {exp }}$, calculated from the reconstructed track position and that observed, $\left(t_{E}-t_{W}\right)_{o b s}$, as a function of plane number. Planes with poor timing calibrations can be identified by the large offsets in $\Delta T$. 
the light output varies from strip to strip. The strip output was mapped for each module at the scintillator factory. However, since then the modules were transported and welded onto the steel planes and it is expected that the light output will have changed. Ultimately, cosmic ray muons will be used to calibrate the strip-to-strip response in the detector, but these constants were not available at the time of writing.

Over suitably small distances, the energy deposited by a muon in a scintillator strip is proportional to the path length in the scintillator. The light produced is attenuated by the wavelength shifting fibre with a characteristic double decay length of $1 \mathrm{~m}$ and $7 \mathrm{~m}$, corresponding to the strong absorption of short wavelengths in plastics and remaining attenuation of longer wavelengths from atomic absorption and scattering from cracks and imperfections in the fibres. Figure 5.12 shows the mean number of photoelectrons, corrected for clear fibre attenuation and muon angle, measured by a photomultiplier tube as a function of the distance between the muon crossing point and the strip end. A slight reduction in light output can be seen at the near strip end due to light capture inefficiencies which have not been fully modelled. There is also a small difference in attenuation lengths observed. Figure 5.13 shows the probability of a track hit being observed on both ends of the strip as a function of the track position on the strip. A difference of the order of $1 \%$ can be seen over the width of the strip. However, when dead chip $\$^{3}$ are accounted for, the discrepancy is reduced to less than 1\%. Figure 5.14 shows the mean charge deposited per unit strip width by a muon. The data shows a broader distribution than the Monte Carlo due to the lack of a strip-to-strip calibration. The lack of strip response calibration is not expected to significantly effect the results of the analysis presented in the following chapters. The measurement of muon momentum from range depends on the topology of the track hits and not on the energy deposited in each strip. Therefore, the calibration solely effects the measurement of the hadronic energy which has an intrinsic resolution of $55 \% / \sqrt{E}$. For the events selected in this analysis the mean hadronic energy is approximately $1 \mathrm{GeV}$. Hence, an additional $15 \%$ uncertainty from the strip to strip calibration makes only a $2 \%$ difference to the hadronic energy resolution in the worse case where all hadronic energy is deposited in a single strip.

\subsubsection{Charge Reconstruction}

Correctly simulating the charge reconstruction requires correct simulation of timing as well as the magnetic field. To remove the effects of the timing simulation discussed

\footnotetext{
${ }^{3}$ see Section 6.3 .5 for a full discussion of how to identify dead channels
} 


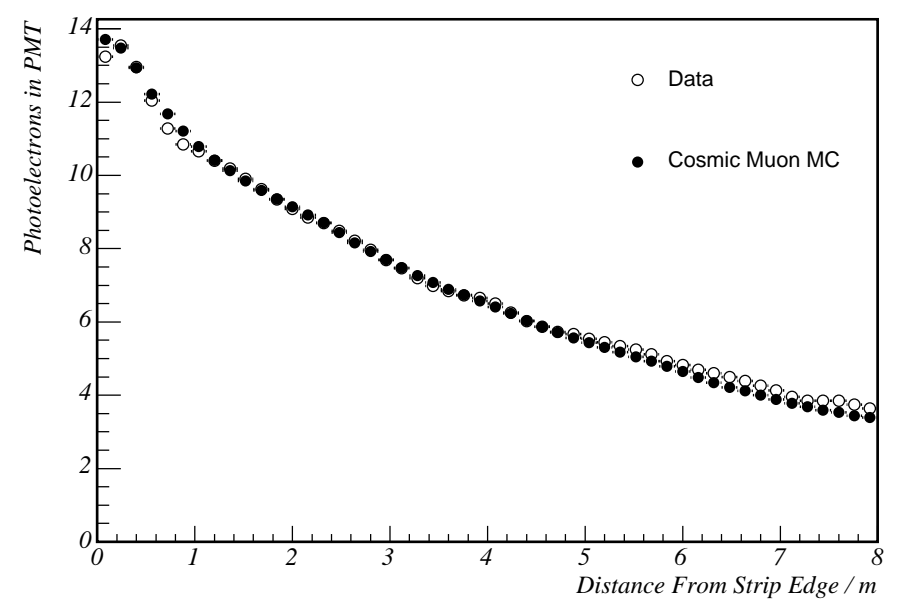

Figure 5.12: The charge recorded by the PMT for a stopping cosmic ray muon as a function of the distance between the muon crossing point obtained from the reconstructed track and the strip end. The path length of the muon through the strip and the attenuation of the signal in the clear fibres have been accounted for.

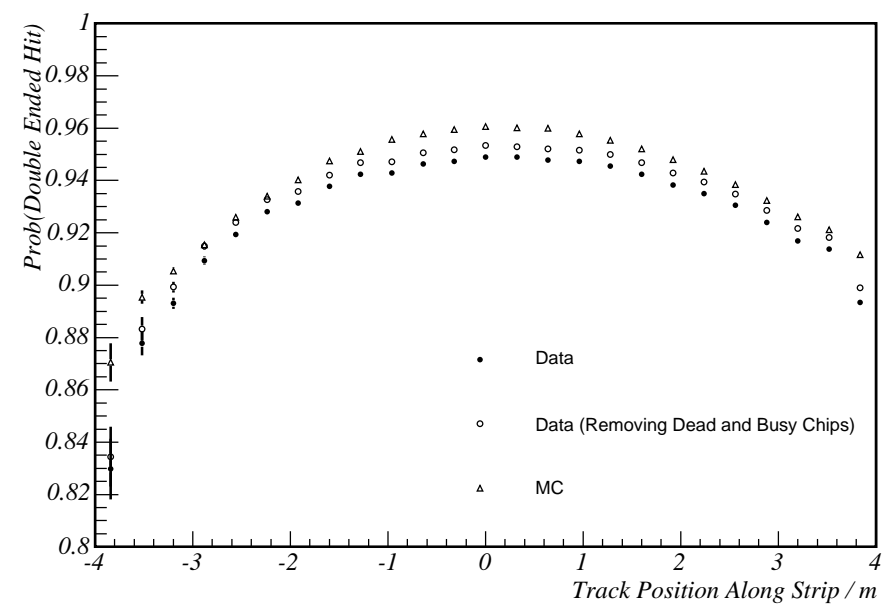

Figure 5.13: The probability of a hit on a cosmic ray muon track being double ended as a function of track position along the strip for stopping cosmic ray muon events. Data and Monte Carlo are shown. Also shown is the probability recalculated for data after removing strips when one of the chips is not reading out or is busy at the time of hit. 


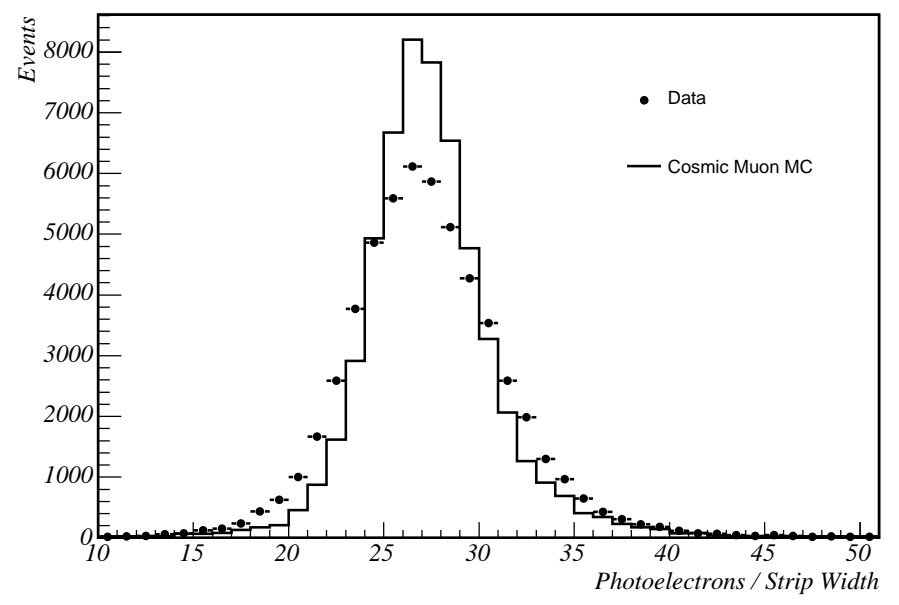

Figure 5.14: The mean charge deposited in scintillator per unit strip width corrected for attenuation in clear and green fibre for stopping cosmic ray muons that span eight planes.

in the previous section, the reconstructed track is required to be downward going and must span a minimum of eight planes. As discussed in Chapter 7, the charge of the muon creating the track is determined by measuring the track's curvature in the magnetic field. The variable $\langle Q / P\rangle / \sigma_{Q / P}$, where $\langle Q / P\rangle$ is the mean charge divided by momentum along the track and $\sigma_{Q / P}$ is the error in the mean, is related to the track curvature and is used to identify the muon's charge. This variable is shown for data and Monte Carlo cosmic ray muons that stop in the detector in Figure 5.15. Two clear peaks can be seen, corresponding to $\mu^{+}$and $\mu^{-}$. However, there is a slight systematic difference between data and Monte Carlo in the heights of the two peaks. The difference is consistent with the $10 \%$ uncertainty on the $\mu^{+} / \mu^{-}$ratio. The measured charge ratio $\mu^{+} / \mu^{-}$for data compared to Monte Carlo as a function of measured muon momentum from the track range is shown in Figure 5.16. The mean value of $1.086 \pm 0.008$ is shown by the dotted line. There is no evidence of a momentum dependant systematic difference between Monte Carlo and data. 


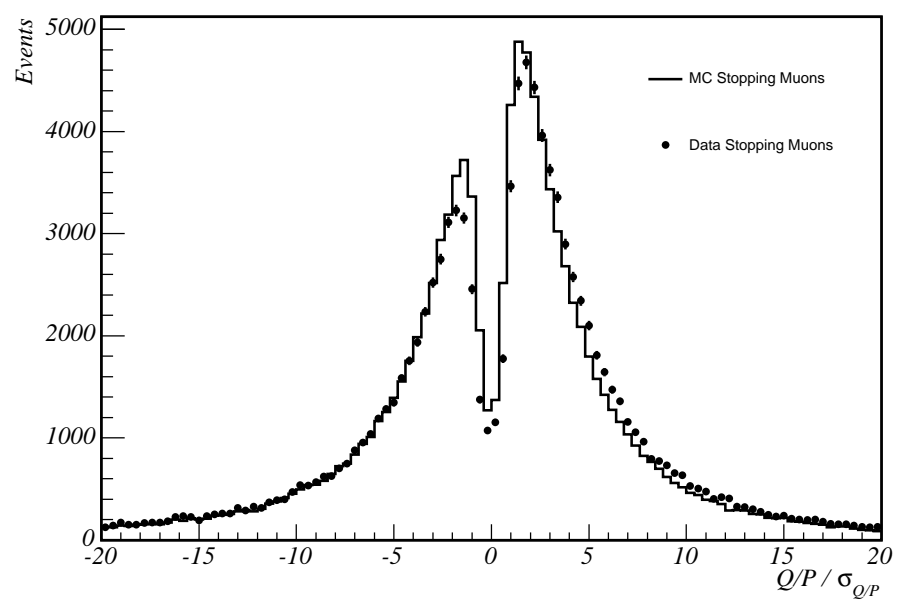

Figure 5.15: The reconstructed value for $\langle Q / P\rangle / \sigma_{Q / P}$ for data and Monte Carlo stopping cosmic ray muons that span more than eight planes and are reconstructed as downward going.

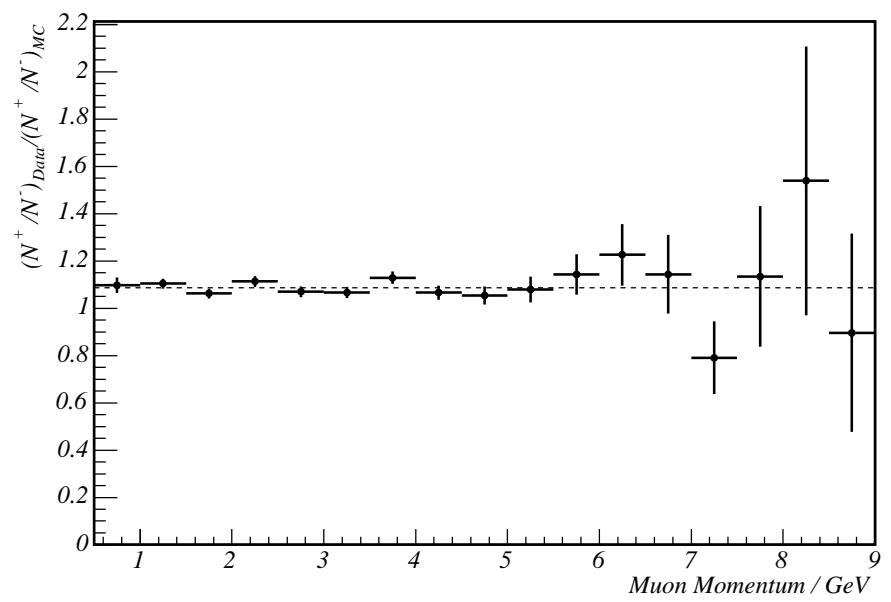

Figure 5.16: The data to Monte Carlo ratio of $\mu^{+} / \mu^{-}$charge ratios as a function of momentum for stopping cosmic ray muons that span more than eight planes and are reconstructed as downward going. The mean value of $1.086 \pm 0.008$ is shown by the dotted line. 


\subsection{Summary}

A large Monte Carlo sample of atmospheric neutrinos in the MINOS Far Detector was generated using the Battistoni flux and the NEUGEN3 interaction model. By combining the systematic uncertainties in the neutrino flux as estimated by the Battistonti group with the estimates of the uncertainties on the neutrino cross sections in the energy range $0.6-10 \mathrm{GeV}$, the uncertainty on the expected number of neutrino interactions is estimated to be $20 \%$.

A large sample (approximately twice the current data exposure) of cosmic ray muons was generated. The sample was compared to cosmic ray muons in the Far Detector. In general the Monte Carlo provides a good description of the data. However, a significant discrepancy was seen between simulated and observed hit timing resolution. This discrepancy is accounted for when interpreting the data, as discussed in Section 7.4.3. The modelling of light output attenuation is accurate to approximately $1 \%$. The variation in light output from strip to strip is significantly larger in data than in Monte Carlo due to the lack of calibration, however, this is not expected to have a significant effect on the analysis. 


\section{Chapter 6}

\section{Signal Selection}

In the absence of neutrino oscillations, the Monte Carlo predicts $415 \pm 83$ triggered $\nu_{\mu} / \bar{\nu}_{\mu}$ charge current (CC) events per year in the MINOS Far Detector. The error is dominated by the systematic uncertainty in the neutrino flux. This rate should be compared to 50000 cosmic ray muon events per day. In order to achieve a contamination of less than $10 \%$ a cosmic ray muon rejection rate of order 1 in $10^{6}$ is required.

A cuts-based selection is used to separate out $\nu_{\mu} / \bar{\nu}_{\mu} \mathrm{CC}$ events from the cosmic ray muon background. The small remaining cosmic ray muon background is measured using the MINOS veto shield. Other sources of background, including neutrons from muon spallation in the surrounding rock and neutrino induced upward-going muons are investigated.

\subsection{Data Selection}

The data presented in this thesis were taken between $1^{\text {st }}$ August 2003 and $1^{\text {st }}$ May 2004 during detector commissioning. Great care has been taken to remove data taken under non-standard conditions.

Through-going muons were used to monitor the detector live time. The distribution of mean through-going muon rates per run for each Supermodule is shown in Figure 6.1. Runs with rates more than 5 standard deviations away from the mean were flagged and removed. The rates of dynode triggers from the shield and the two Supermodules before the application of the DAQ triggers were monitored on a per second basis. Runs with anomalously low trigger rates from the veto shield or either supermodule were removed. It was required that the coil in both Supermodules be 


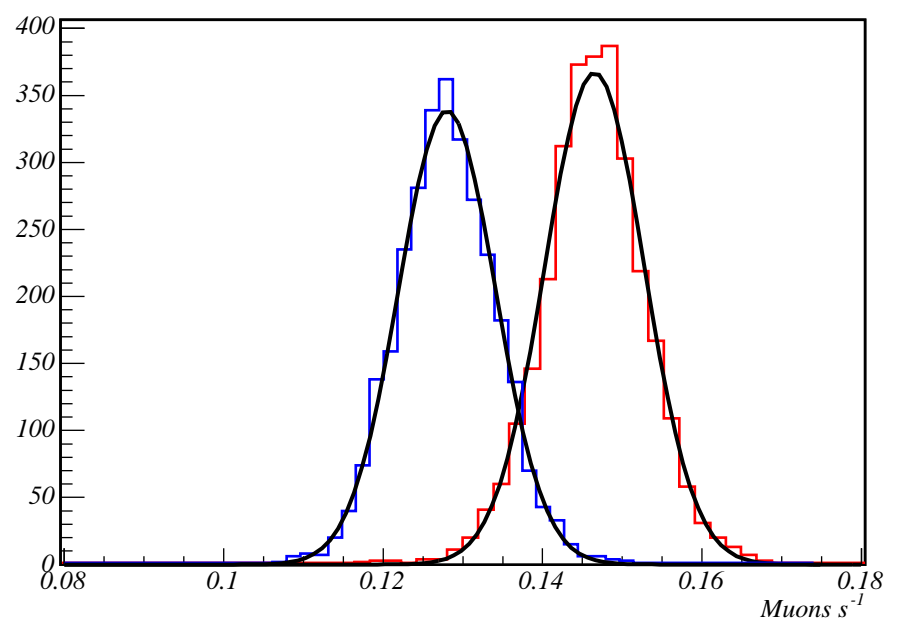

Figure 6.1: The mean rate of through going muons in Supermodule 1 (red) and Supermodule 2 (blue) per run, The vertical axis shows the number of runs. The mean through-going muon rates are $0.1465 \mathrm{~Hz}$ for Supermodule 1 and $0.1280 \mathrm{~Hz}$ for Supermodule 2 .

switched on and running at normal current. All high voltage supplies were required to be running at operating voltages.

The total exposure time of the data presented is 4113.2 hours, or 2.52 kton-years. The run time was calculated from the number of timeframes processed by the trigger farm and is known to an accuracy of one second.

\subsection{Event Reconstruction}

The 'standard' MINOS reconstruction software has been optimised for beam neutrino events in which the neutrino is travelling approximately parallel to the $Z$-axis of the detector. The analysis presented here uses reconstruction software developed by the Cambridge group that has been optimised for atmospheric neutrino events.

The first step of reconstruction is the removal of the eight-fold ambiguity in the association of raw hits to strips, a process referred to as demultiplexing. This is performed using the AltDeMux package [89] which uses information from both strip ends and hit timing to remove the ambiguities. For cosmic ray muon Monte Carlo, more than $99 \%$ of the charge in the event is assigned to the correct strip. Fake hits resulting from optical and electrical cross-talk are identified and removed at this stage.

After demultiplexing, the data is in the form of two $2 \mathrm{D}$ event views, $U-Z$ and $V-Z$. The AtNu package [90] is used to reconstruct the two event views into 3D tracks and 
showers. Figure 6.2 shows the percentage of triggered $\nu_{\mu} \mathrm{CC}$ events for which the tracking software has found the muon track. At low energies the muon does not cross enough planes for the tracker to identify it. At higher energies the efficiency levels off at $74 \%$, the rejected events are either events where the muon track is obscured by the vertex shower or the muon has exited the detector leaving a short track.

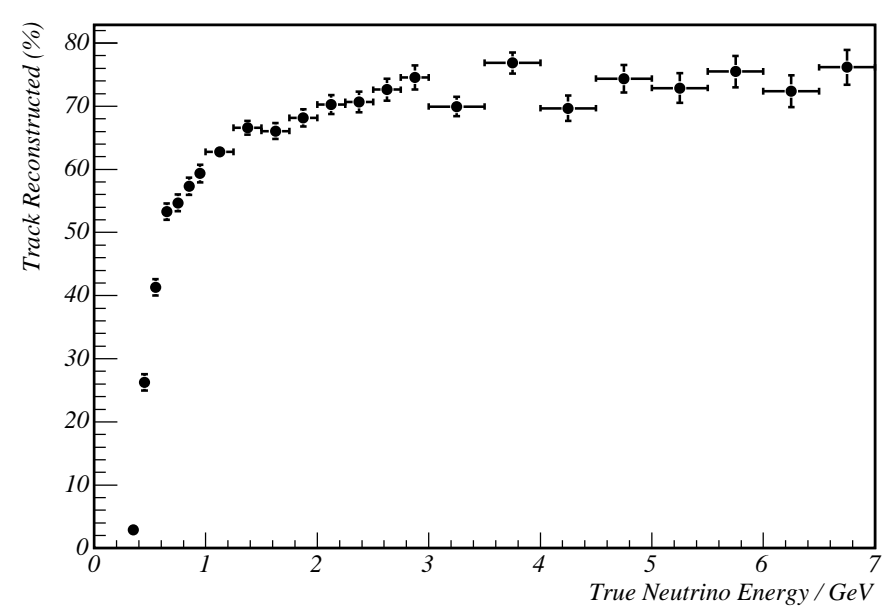

Figure 6.2: The efficiency, from Monte Carlo, of muon tracking for triggered $\nu_{\mu} \mathrm{CC}$ events.

\subsection{Background Rejection}

A series of seven selection cuts was developed with the aim of increasing the signal to background ratio to approximately 1 to 1 before using information from the veto shield. The cuts were developed using cosmic ray muon and atmospheric neutrino Monte Carlo samples, discussed in the previous chapter.

\subsubsection{Track Length}

The tracking software requires hits in at least six planes to reconstruct a track and may produce multiple tracks per event. The majority of $\nu_{\mu} \mathrm{CC}$ events have zero or one track reconstructed. The contribution from multi-tracked events is less that $0.5 \%$. Only single tracked events are considered in this analysis.

Events are only considered if the track spans eight or more planes for two reasons. Firstly, the fraction of atmospheric neutrinos reconstructed as single tracked events 
that are $\nu_{\mu} \mathrm{CC}$ interactions increases with increasing number of planes spanned, as shown in Figure 6.3(a). Secondly, the muon tracking is better for events that span more planes. Figure 6.3(b) shows the fraction of hits on the reconstructed track that are caused by the muon for $\nu_{\mu} \mathrm{CC}$ events as a function of the number of planes spanned by the track. This cut introduces a lower limit on the momentum of the muon of $400 \mathrm{MeV}$, the momentum required for a muon to pass through 8 planes at normal incidence.

\subsubsection{Containment}

A fiducial volume that contains $73 \%$ of the detector mass is defined. Events that show significant activity outside this volume are rejected. Events which start or stop within the first or last five planes of a Supermodule are rejected, a five-plane-veto. Figure 6.4(a) shows the number of planes between the end of a supermodule and the closest end of a track. The slight depletion of events in the second to fourth bins is due to the minimum track length requirement.

Events that enter the detector via its sides are removed by requiring that the minimum distance between either end of the track and the detector's side, the fiducial distance, be greater than $50 \mathrm{~cm}$. Figure 6.4(b) shows the fiducial distance before the application of a five-plane-veto. A clear peak in the fiducial distance can be seen at approximately $3.75 \mathrm{~m}$ from cosmic muons that enter the detector via the uninstrumented coil hole. These events are removed by requiring both ends of the track to be more than $40 \mathrm{~cm}$ from the centre of the detector in the $X Y$-plane.

A containment cut is also applied at the hit level. To obtain the position of each hit in 3 dimensional space, the position in the orthogonal view is obtained from the mean position of the charge in the two adjacent planes. Events with a charge of more than 5 photoelectrons outside the fiducial volume are rejected. Figure 6.5 shows the total charge outside the fiducial volume for Monte Carlo cosmic ray muons and data. The containment cuts remove $99.8 \%$ of the cosmic ray muons.

\subsubsection{Track Quality}

Events where the reconstructed track is embedded in a shower were rejected by requiring the track be clean. A clean track is required to have six or more out of any contiguous group of 7 planes where more than $50 \%$ of the charge in that plane is within \pm 1 strip of the track position. Clean tracks are also required to have a maximum pulse height in any plane which is less than 350 photoelectrons (corresponding to a hadronic energy of $1.5 \mathrm{GeV}$ ). This removes events where the cosmic ray muon travels parallel to the planes, depositing a large amount of energy in a single plane. 


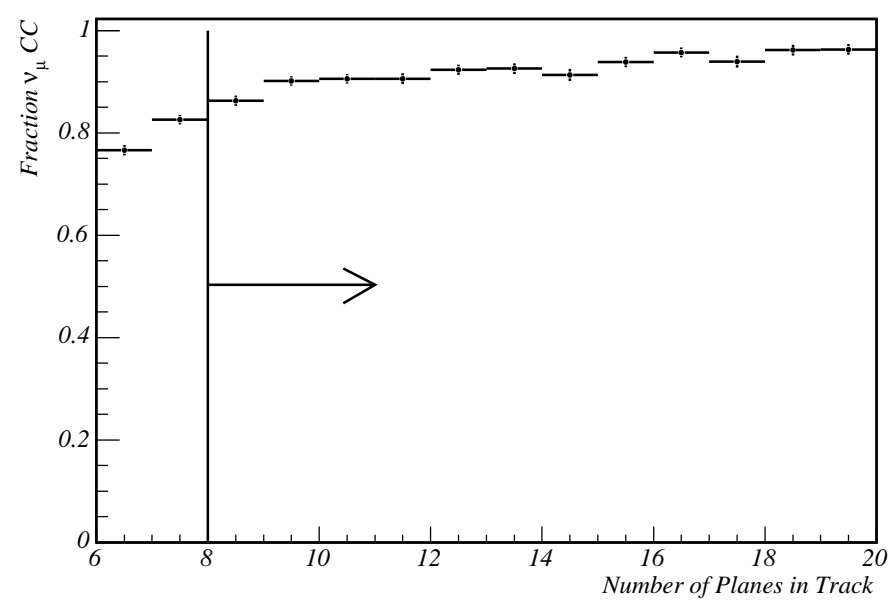

(a)

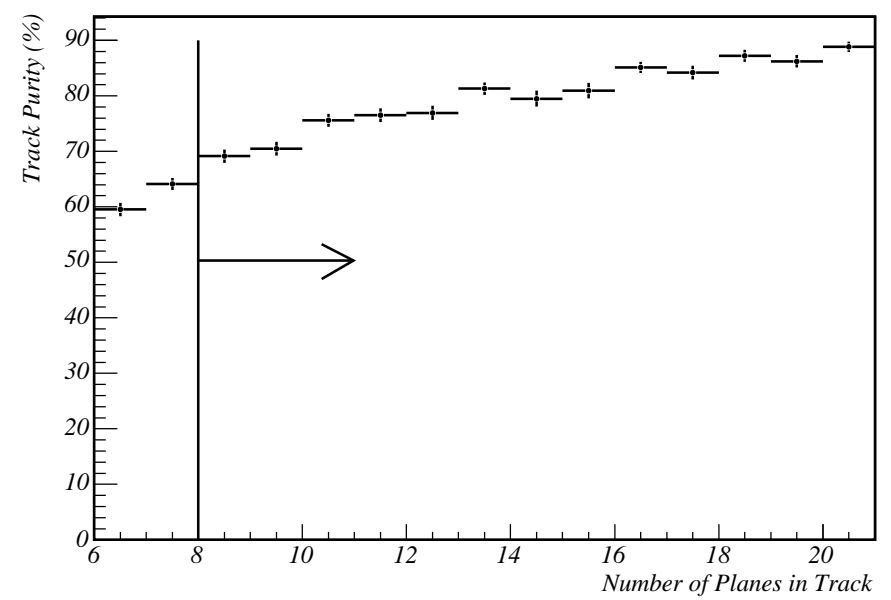

(b)

Figure 6.3: (a) The fraction of Monte Carlo atmospheric neutrinos reconstructed as single tracked events that are $\nu_{\mu}$ CC interactions. (b) The percentage of hits along a track that are due to a muon for single tracked $\nu_{\mu}$ CC events as a function of number of planes spanned by the track. 


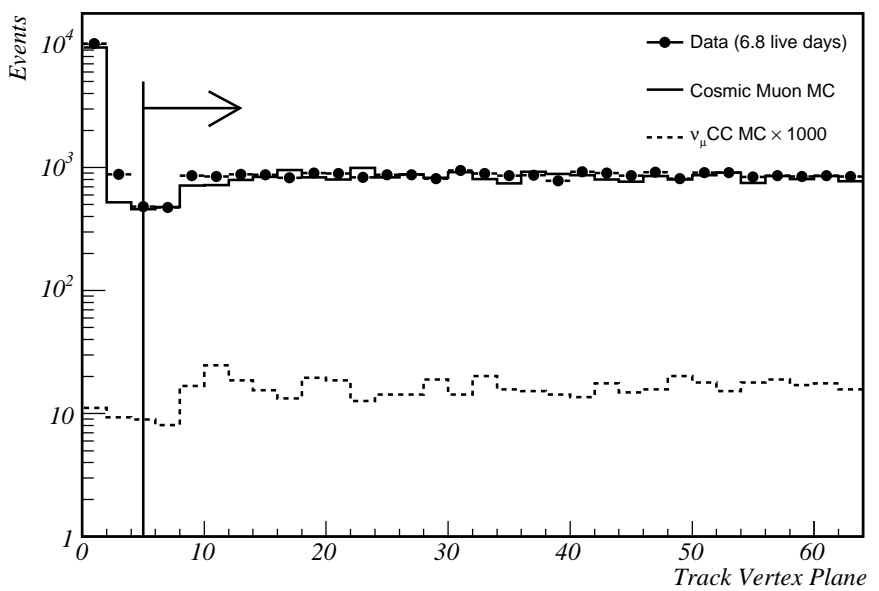

(a)

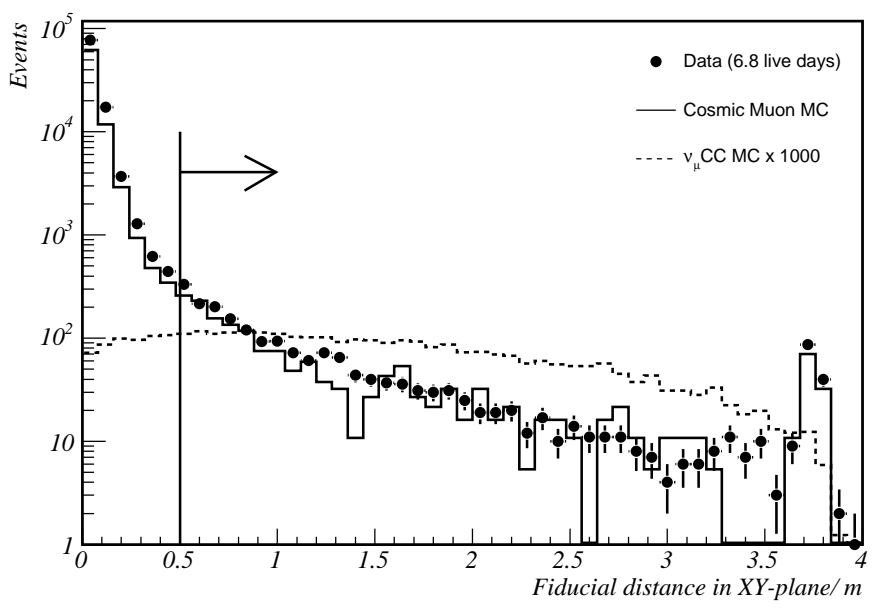

(b)

Figure 6.4: (a) The plane of the reconstructed vertex from timing. A 5 plane cut is made at the boundary of both Supermodules. The same requirement is made on both track ends. (b) The fiducial distance. A cut is made at $50 \mathrm{~cm}$. Data from 6.8 live days is shown by the points, Monte Carlo cosmic ray muons are shown by the solid line and $\nu_{\mu} \mathrm{CC}$ events are shown by the dashed line. 


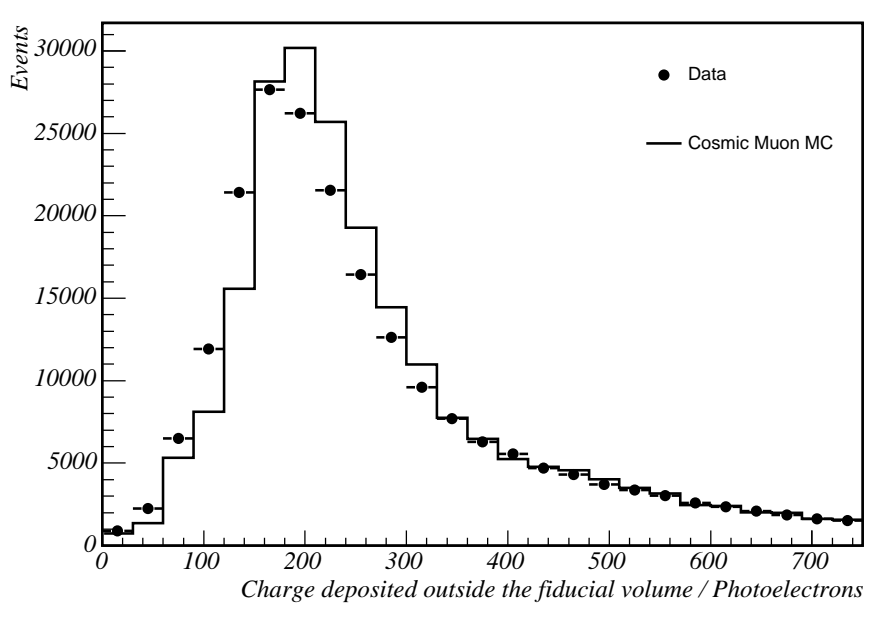

(a)

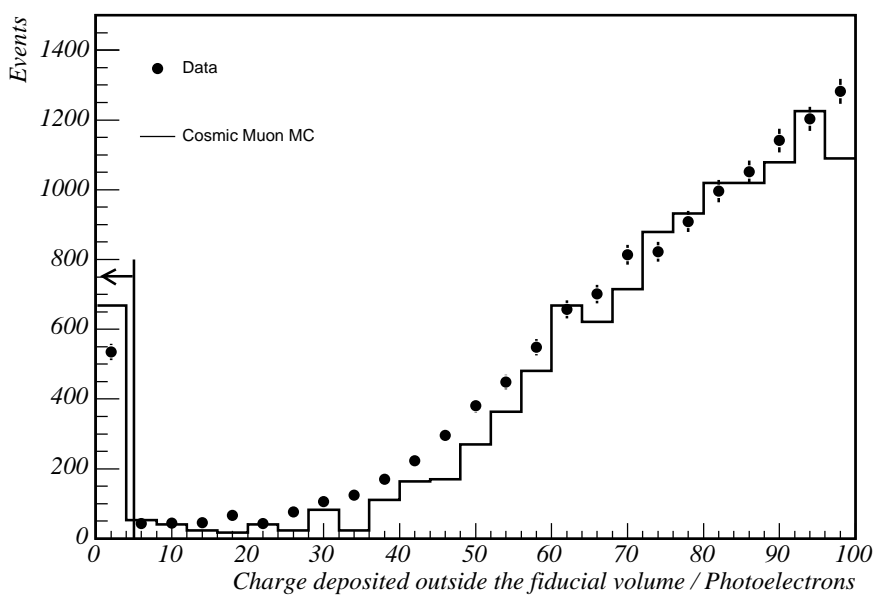

(b)

Figure 6.5: (a) The total charge deposited outside the fiducial volume for 6.8 live days of data compared to Monte Carlo predictions for cosmic ray muons. (b) Expanded view of the low charge region. A cut is made at 5 photoelectrons. 


\subsubsection{Cosmic Ray Muon Rejection}

After the above requirements, the background is dominated by steep cosmic ray muons that enter the detector at small angles to the $X Y$-plane. These muons can penetrate several meters into the fiducial volume before traversing a scintillator plane and leaving a detectable signal. The event rate from this class of events is approximately 15000 events year ${ }^{-1}$. All cosmic ray muon events are downward-going, therefore, when considering cosmic muon rejection the upper track end, i.e. the one with the largest $Y$ co-ordinate is taken to be the vertex. This ensures that inefficiencies in the background rejection are not introduced by inefficiencies in event direction reconstruction.

To identify this class of event the track is extrapolated linearly from the upper track end to the edge of the detector. The $Z$ projection of this extrapolation is calculated, $\Delta_{Z}$, and is termed the event trace. For high energy, well reconstructed muons, $\Delta_{Z}$ should approach the distance between active planes, approximately $6 \mathrm{~cm}$. However, for low energy events the muon is deflected by the magnetic along the $Z$ direction, increasing $\Delta_{Z}$. Figure 6.6 shows the mean $\Delta_{Z}$ as function of the true muon energy for Monte Carlo cosmic muons. Figure 6.7 shows the distribution of $\Delta_{Z}$ for data, signal and Monte Carlo background for all single track events that pass the containment and track quality cuts. Good agreement is seen between data and Monte Carlo. The requirement that the reconstructed $\Delta_{Z}$ be greater than $50 \mathrm{~cm}$ is made, reducing the cosmic muon background rate from 15000 to 2800 events per year. The corresponding loss in signal is $4 \%$.

After the $\Delta_{Z}$ cut, $30 \%$ of the remaining background consists of steep cosmic ray muons that pass the cut due to the difficulty in reconstructing steep tracks with high curvature in the first few planes. These events tend to deposit a large charge in the first active plane they cross as they traverse many scintillator strips due the steep trajectory. Figure 6.8 shows the charge in the vertex plane, $Q_{v t x}$, against the cosine of the zenith angle, $\cos \theta_{z e n i t h}$. For robustness against reconstruction errors $Q_{v t x}$ is taken to be the maximum charge in the first 4 planes from the upper end of the track. Further discriminating power comes from the fact that the remaining cosmic ray background travels at small angles to the planes, i.e. at small values of $\left|\cos \theta_{Z}\right|$. Figure 6.9 shows $\left|\cos \theta_{Z}\right| / Q_{v t x}$ against $\cos \theta_{\text {zenith }}$ for both $\operatorname{cosmic}$ ray muon and signal Monte Carlo. The variable $\eta$, defined as

$$
\eta=60 \frac{\left|\cos \theta_{z}\right|}{Q_{v t x}}-\cos \theta_{z e n i t h}
$$




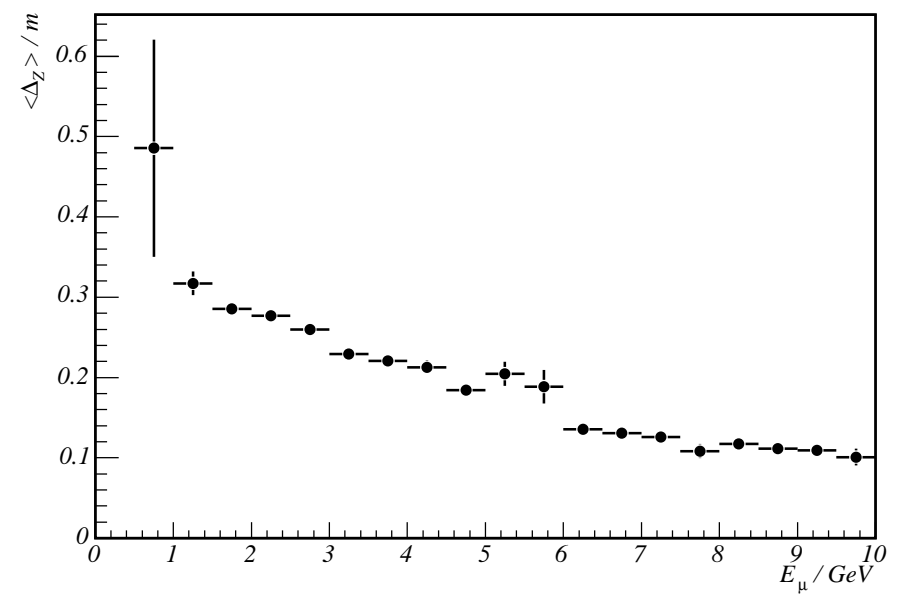

Figure 6.6: The mean $\Delta_{Z}$ of the Monte Carlo cosmic ray muon background against the true muon energy for contained events that pass the track quality cuts.

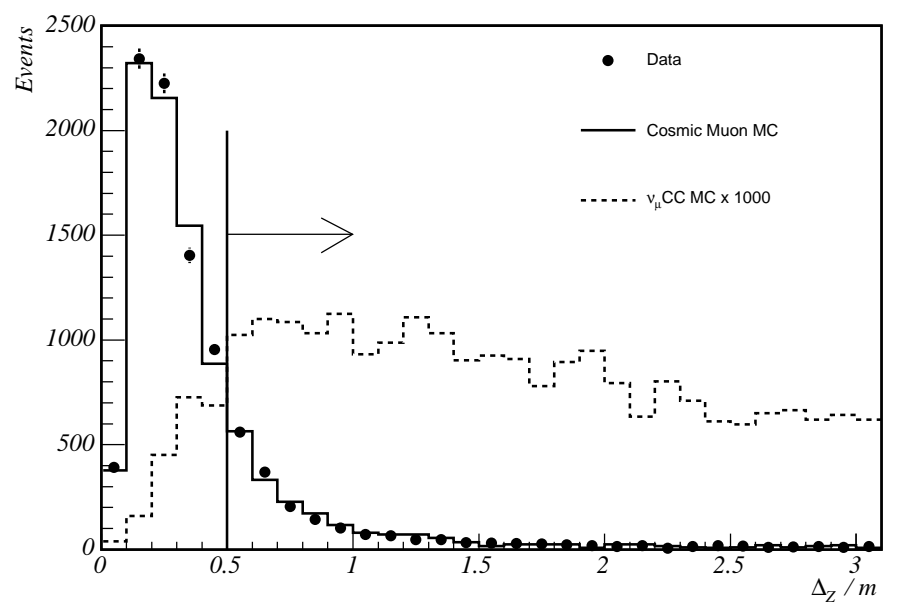

Figure 6.7: The trace for Monte Carlo cosmic ray muons (solid line), $\nu_{\mu}$ CC Monte Carlo (dashed line) and 2.52 kton-years of data (points). 
is constructed. Figure 6.10 shows the distribution of $\eta$ for data compared to Monte Carlo cosmic ray muons. The expected distribution for signal events is also shown. Events are rejected if $\eta<0$.
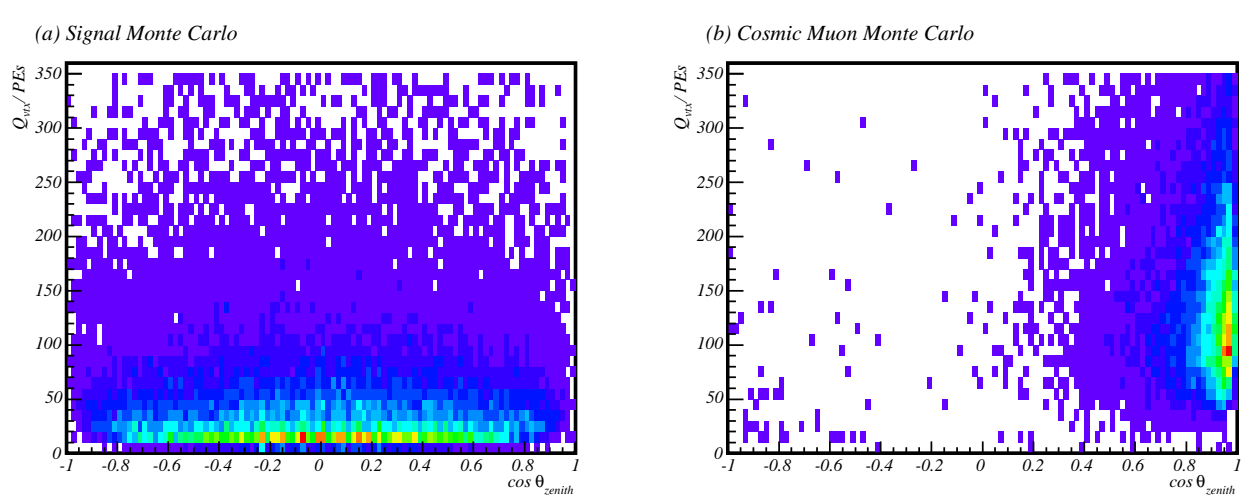

Figure 6.8: The Monte Carlo distributions $Q_{v t x}$ plotted against $\cos \theta_{\text {zenith }}$ for cosmic ray muon background and the $\nu_{\mu} \mathrm{CC}$ signal events, for events that pass containment and track quality cuts.
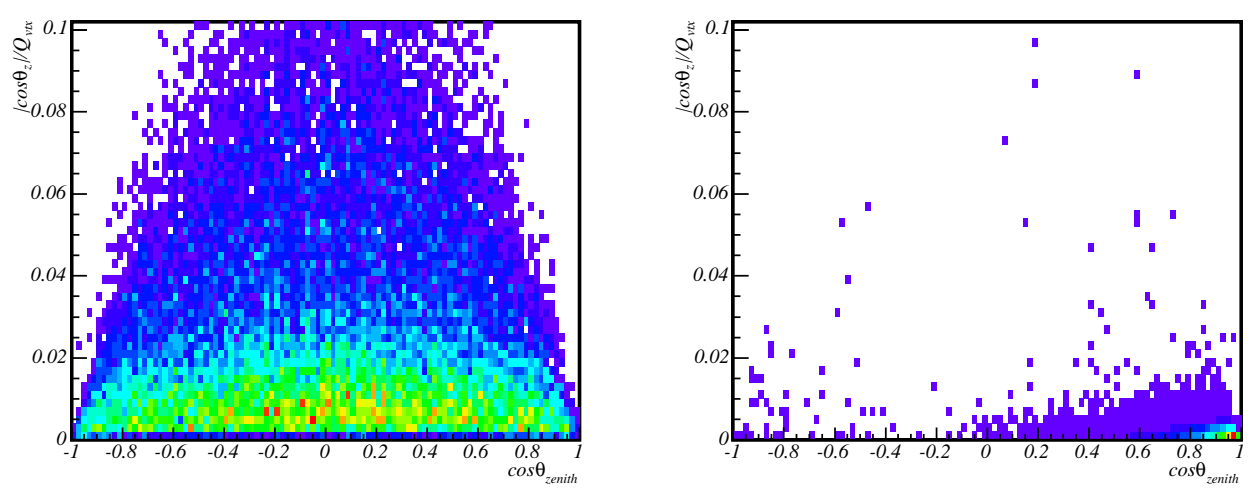

Figure 6.9: The Monte Carlo distributions of $\cos \theta_{z} / Q_{v t x}$ against reconstructed $\cos \theta_{z e n i t h}$ for signal events (left panel) and cosmic ray muon background (right panel), for events that pass containment and track quality cuts.

After the trace cut, $70 \%$ of the remaining background are events in which the muon has undergone a large deflection in the magnetic field such that it has passed through at least one scintillator plane twice in opposite directions, leaving hits in two positions in that plane. Demultiplexing planes with two muon hits is significantly harder than planes with a single hit. In addition, the reconstruction software only allows a track to pass through a plane once, so only chooses one of the solutions for 


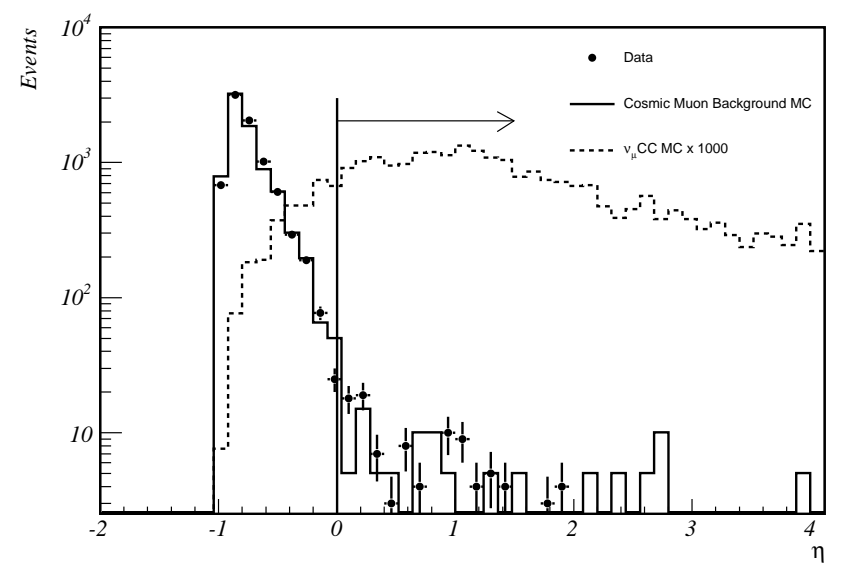

Figure 6.10: The Monte Carlo distribution of the discriminant variable $\eta=60 \frac{\left|\cos \theta_{z}\right|}{Q_{v t x}}-$ $\cos \theta_{z e n i t h}$, for events that pass the containment and track quality cuts.

the track location. As it is required that the reconstructed track be contained, the selection is biased towards events where the tracker has chosen the lower of the two solutions. Figure 6.11 shows an example of this class of event. These events typically deposit a significant amount of charge in plane at the end of the event, where the event turns over as it has a longer path length in the scintillator. Therefore, $93 \%$ of these are rejected by the $\eta$ cut. The remaining $7 \%$ are identified by the charge weighted distribution of the hits from the fitted track in the $U-Z$ and $V-Z$ views. The mean charge weighted distance of the hits from the track location in both views,

$$
\left\langle\Delta_{U V}\right\rangle=\left(\sum_{i}^{U \text { view hits }} Q_{i}\left(U_{i}^{\text {track }}-U_{i}^{\text {hit }}\right)+\sum_{i}^{\text {View hits }} Q_{i}\left(V_{i}^{\text {track }}-V_{i}^{\text {hit }}\right)\right) / \sum_{i} Q_{i}
$$

of the hits about the track position in each plane, and its root mean square deviation, $\left\langle\Delta_{U V}^{2}\right\rangle^{\frac{1}{2}}$, are calculated. Selected events are required to have $\left\langle\Delta_{U V}\right\rangle>-0.25 \mathrm{~m}$ and $\left\langle\Delta_{U V}^{2}\right\rangle^{\frac{1}{2}}<0.8 \mathrm{~m}$, where a negative value of $\left\langle\Delta_{U V}\right\rangle$ indicates that most of the charge was above the track, i.e. at larger $U$ or $V$ co-ordinates. Figure 6.12(a) and Figure 6.12(b) show good agreement between data and Monte Carlo for both $\left\langle\Delta_{U V}\right\rangle$ and $\left\langle\Delta_{U V}^{2}\right\rangle^{\frac{1}{2}}$.

After the cosmic ray muon rejection cuts 91 events were selected for a Monte Carlo expectation of 103 in the absence of oscillations. Table 6.1 shows the number of observed and expected events at each stage in the event selection. 

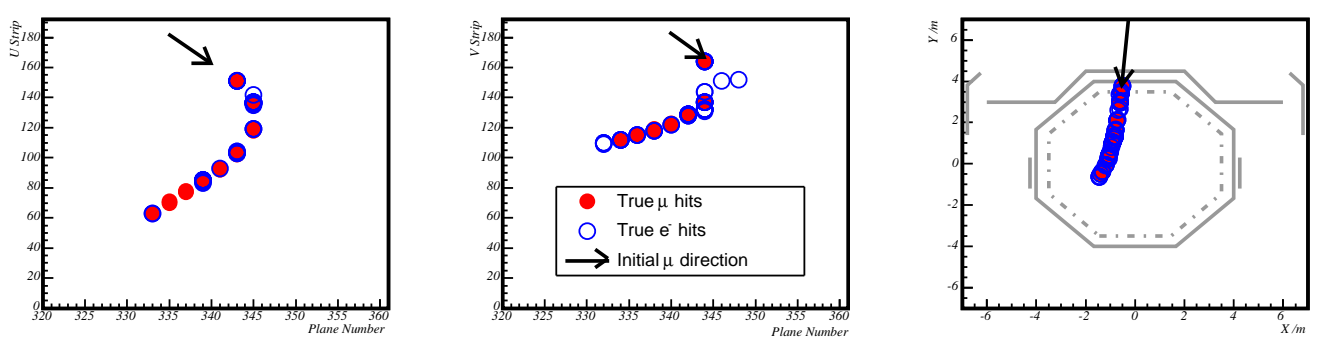

(a)
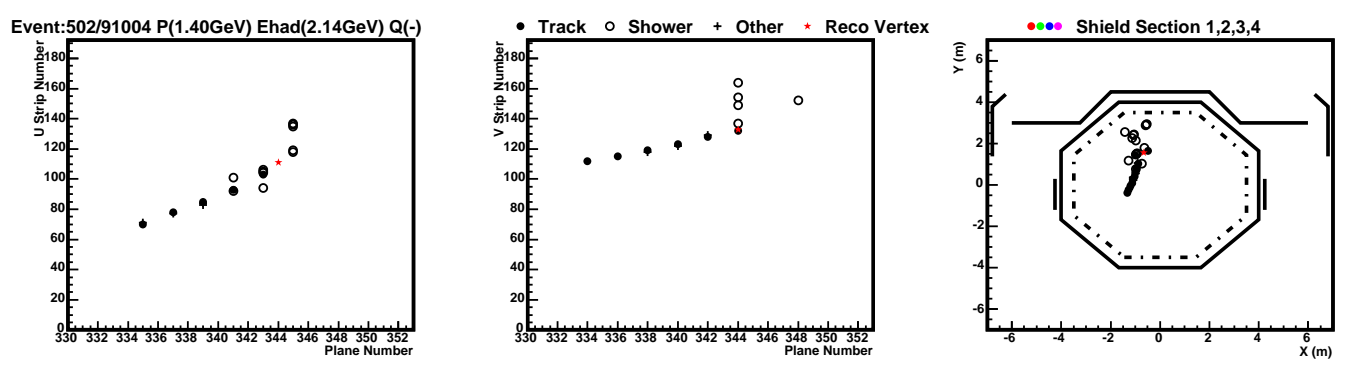

(b)

Figure 6.11: An example of a simulated cosmic ray muon event that has undergone a significant deflection in the magnetic field so that it changes its direction of travel along the $Z$ axis . (a) The initial direction of the muon is shown by the arrows, the true location of muon hits are shown by the solid dots and true electron hits are shown by open markers. (b) The hit locations after demultiplexing. In some planes, for example plane 344, the demultiplexer has grouped the two hit clusters together into one, in other planes it finds the correct solutions, for example plane 345 . The hits chosen by the tracking software are shown by the filled circles. As the software does not allow for more than one position in a plane it stops tracking after plane 345 . All other hits are assumed to be a vertex shower. The event can be identified by the mean and RMS of the position of the hits about the track in the $U-Z$ and $V-Z$ views. 


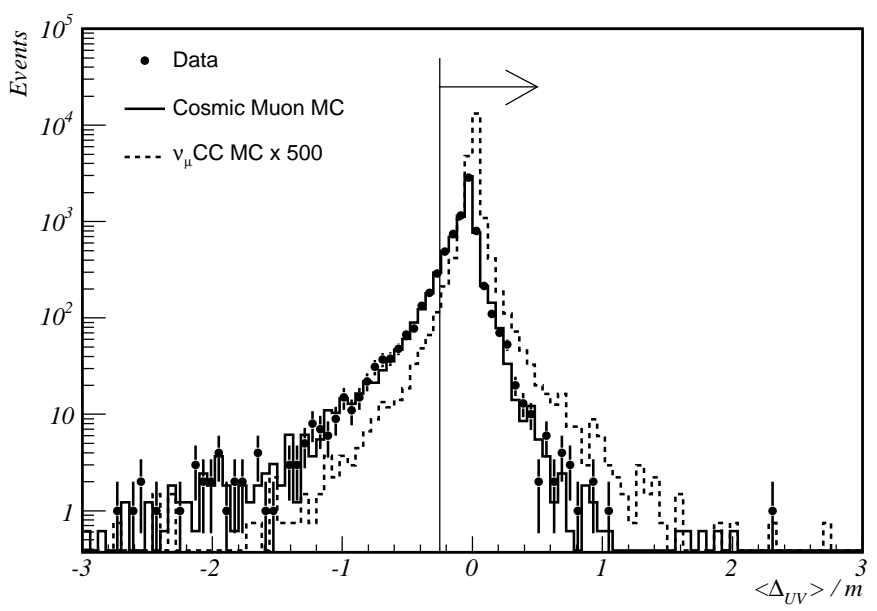

(a)

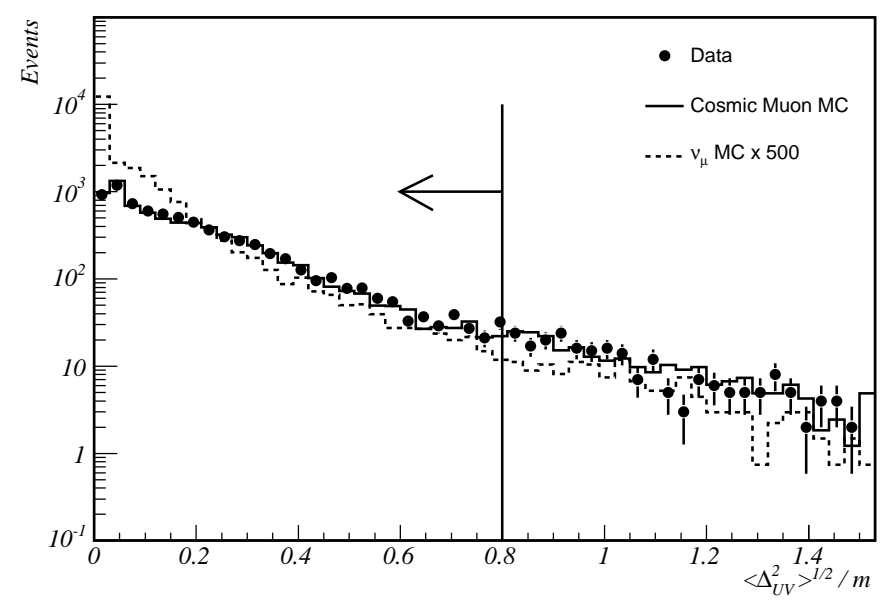

(b)

Figure 6.12: (a) The mean hit location about the track position, $\left\langle\Delta_{U V}\right\rangle$. (b) The RMS of the hit locations about the track position, $\left\langle\Delta_{U V}^{2}\right\rangle^{\frac{1}{2}}$ for events that pass the containment and track quality cuts. 


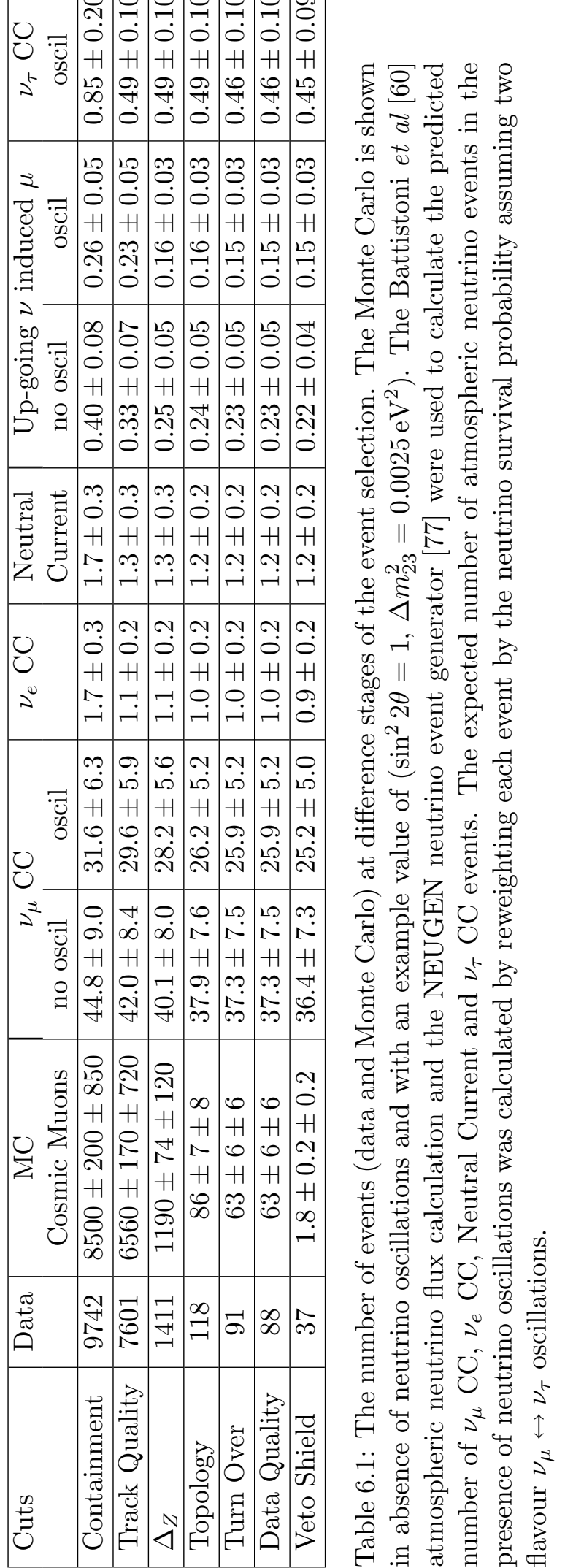




\subsubsection{Event Quality}

In addition to the cut described above the remaining events were examined for evidence of detector related effects that may cause additional backgrounds.

\section{Timeframe Boundaries}

The DAQ trigger farm processes data in 1 second timeframes. There is a small probability $\left(1\right.$ in $\left.5 \times 10^{6}\right)$ that events will be split across timeframe boundaries breaking the physics event into two reconstructed events. Ultimately, the DAQ system will be upgraded to process timeframes that overlap by $1 \mu \mathrm{s}$, eliminating this effect. However, the upgrade was not installed in time for the data used in this analysis. To remove split events, the event trigger time was required to be at least $1 \mu \mathrm{s}$ from the edge of the timeframe, with an expected rejection rate of 1 in $10^{6}$ events. Two data events that passed all other cuts were rejected by this requirement.

\section{Dead Channels}

A single dead plane can cause a steep muon to appear as a contained event if the hits near the muon entry point are lost. It is therefore, important to identify events that may have been truncated due to dead detector planes. There are two possible causes for a dead channel.

1. Busy Chips. It takes $5 \mu \mathrm{s}$ for the analogue-to-digital converters (ADC) to read out a single VA chip and each ADC digitises the signals from six chips. Therefore, it can take up to $30 \mu$ s between a dynode trigger and the chip being read out, during which time further dynode triggers are ignored. A single PMT reads out two thirds of one side of a plane (128 strips).

There are two possible causes of a busy chip: detector noise and the lightinjection calibration system. To identify chips that are busy due to noise, the previous $30 \mu \mathrm{s}$ of detector activity, called the pre-trigger window, is written out with each event 1 . The hits in the pre-trigger window are time ordered according to their dynode trigger time. The analysis software then calculates when the chip will have finished reading out and will become available again. Chips that are still reading out at the time of the first hit associated with the event are

\footnotetext{
${ }^{1}$ The first month, or 300 hours, of data used in this analysis used a pre-trigger window of $5 \mu \mathrm{s}$ rather than $30 \mu \mathrm{s}$. However, less that $10 \%$ of these events are expected to have busy chips due to hits before the $5 \mu \mathrm{s}$.
} 
flagged as dead. On average 6.14 chips were dead per event, corresponding to 4.25 planes with one strip end dead. The probability of there being one or more planes dead on both sides was obtained from data and found to be $11 \%$ and is believed to be dominated by $\gamma$ decays.

The calibration system injects light into the strip ends at $50 \mathrm{~Hz}$. While light is being injected the chips that readout both ends of the strip being injected will be unavailable to read out a physics event. All channels in a crate are calibrated in parallel and each crate is calibrated in turn. Each crate reads out 64 planes, therefore, $13 \%$ of the active detector is dead on both sides for $0.15 \%$ of all physics events when light-injection is running. Hits from the light injection system are identified and removed from the data stream by the trigger processors. Short summaries of times that each crate was calibrated are written to file with the data. The summaries are used by the analysis software to flag events that have be truncated by light-injection.

2. Malfunctioning Electronics. Failed electrical components are only replaced during scheduled detector down time. The DAQ records the rate of dynode triggers in each chip and these summaries are then used to identify parts of the detector that are not reading out. The mean rate of dynode triggers per chip is $5 \mathrm{kHz}$, if the rate on any chip falls below $0.1 \mathrm{kHz}$ it is flagged as dead. During the data used in this analysis there were an average of two chips not reading out.

For the purposes of selecting atmospheric neutrinos, it is required that there is no more than a single dead plane at either end of the event. One data event that passed all other cuts failed this requirement.

\subsection{The MINOS Veto Shield}

After the above cuts, 88 data events remain, Table 6.1 shows the predicted number of events of each class. The remaining cosmic ray muon background is tagged using the veto shield that covers the upper part of the detector. An event is vetoed if there was charge deposited in the section of the shield above the upper end of the track within $100 \mathrm{~ns}$ of the event. This time window is 20 times the resolution of the shield timing discussed in Chapter 2. However, the stated resolution assumes good reconstruction. By opening out the window to $100 \mathrm{~ns}$, contained events arising from reconstruction failures can also be tagged. 


\subsubsection{Background Rejection Efficiency}

The efficiency of the veto shield for tagging the remaining cosmic ray muon background was estimated by two independent methods. The first method involved using a sample of muons that stop in the detector. A series of four cuts was applied to select out stopping cosmic ray muons that occupy a similar phase space to the remaining cosmic ray muon background in the analysis.

- Track Length: The reconstructed track was required to span at least 8 planes.

- Containment Cuts: The reconstructed track vertex was required to be outside the fiducial volume and the end of the track was required to be inside. Muons that enter the detector via the coil hole are removed by requiring that the reconstructed radial position of the vertex be greater than $40 \mathrm{~cm}$. Muons that leave the detector in the air gaps or steel planes, and therefore leave no signal outside the fiducial volume, are removed by a $30 \mathrm{~cm}$ trace cut applied to the end of the track.

- End Plane Cut: It is required that the reconstructed track does not start or stop in the first five or last five planes of each supermodule. The dominant source of cosmic ray muon background is from muons that enter the detector between planes, not from the detector ends.

- $\cos \theta_{Z}$ Cut: Figure 6.13 shows the cosine of the true angle of the muon to the $X Y$ plane, $\cos \theta_{Z}$, for the cosmic ray muon background after the containment cuts. To obtain a set of stopping cosmic ray muons that occupy the same phase space, selected events are required to have $\left|\cos \theta_{Z}\right|<0.25$.

In the 170.4 live days of data taken, 116000 stopping muons were selected, 18000 passed the $\cos \theta_{Z}$ cut and were used to estimate the shield background rejection efficiency.

Veto shield hits are only considered if they are in the shield sections directly above the end of the event with the highest $Y$ co-ordinate. In regions of the detector where shield sections overlap, both sections are considered. The event is vetoed if the summed charge from selected shield hits is greater than 2 photoelectrons. Table 6.2 shows the shield efficiencies for both supermodules, measured using the stopping muon sample. The veto shield cut rejected $97.1 \pm 0.1 \%$ of the steep cosmic muons. Figure 6.14(a) shows that the shield efficiency is stable through the period that the data were taken. 


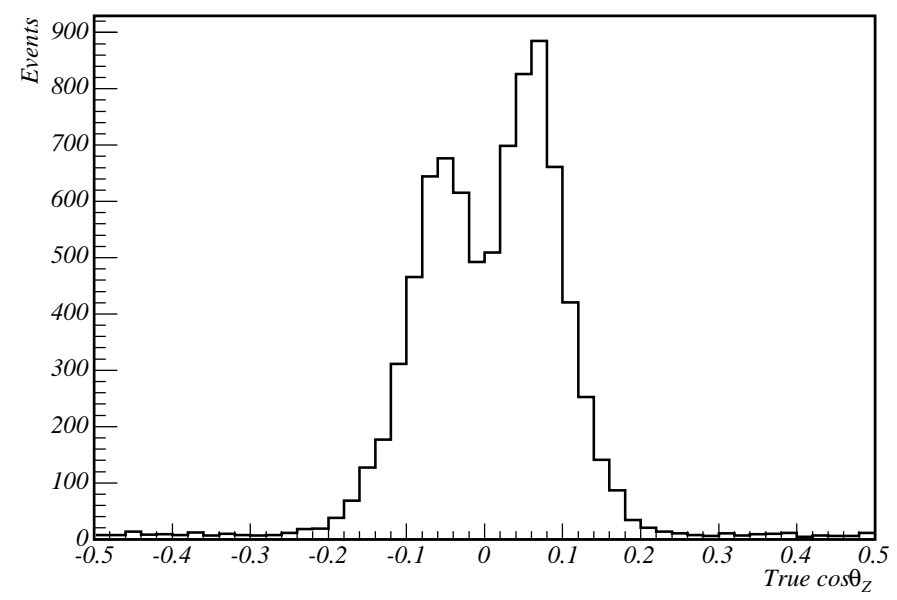

Figure 6.13: The true $Z$ direction cosine of the cosmic muon background after containment cuts.

\begin{tabular}{|c|c|}
\hline & Shield Efficiency $\%$ \\
\hline Supermodule 1 & $97.6 \pm 0.2$ \\
\hline Supermodule 2 & $96.5 \pm 0.2$ \\
\hline
\end{tabular}

Table 6.2: The veto shield background rejection efficiencies for each supermodule calculated using steep, stopping cosmic muons

A second estimate of the veto shield efficiency was obtained by relaxing the event selection cuts until the sample was dominated by background. The $\eta, \Delta_{U V}$ and $\Delta_{Z}$ cuts were relaxed until 2191 events were selected for an expected signal of $29.9 \pm 6.0$ events (assuming $\Delta m^{2}=0.0025 \mathrm{eV}^{2}$ ). Applying the same veto shield requirements used in the stopping muon analysis, $94.4 \pm 0.5 \%$ of the events were rejected. When the expected signal is removed, the estimated shield efficiency is $97.2 \pm 0.4 \%$, which is consistent with the results shown in Table 6.2. From the two independent methods described above, the shield efficiency is estimated to be $97.1 \pm 0.1$ (stat.) \pm 0.4 (sys.), where the central value is taken from the stopping muon analysis and the systematic error reflects the difference between the two methods.

\subsubsection{Signal Inefficiencies}

Contained $\nu_{\mu} \mathrm{CC}$ events may be rejected by the veto shield cuts either because of random noise in the shield or from shield hits associated with the event. 


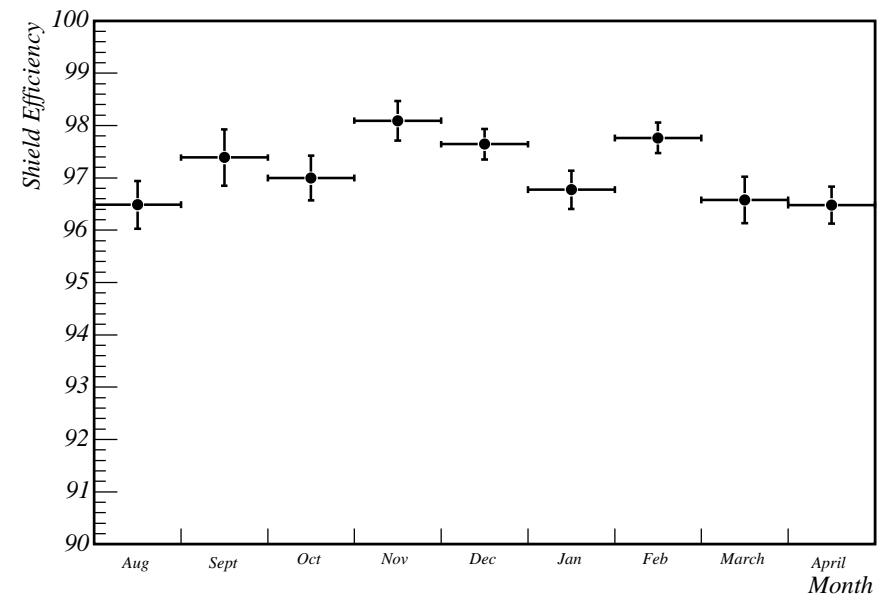

(a)

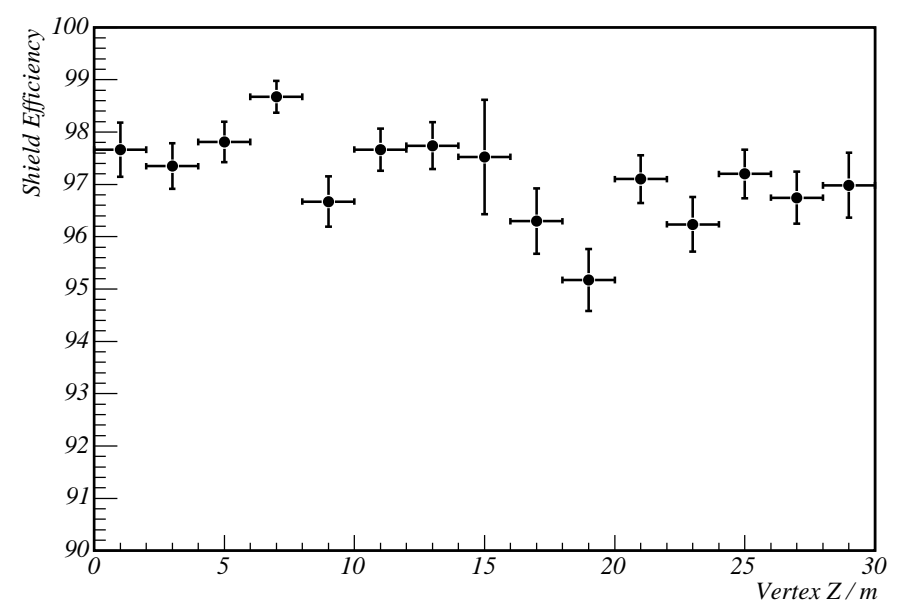

(b)

Figure 6.14: The veto shield tagging efficiency for stopping muons, (a) for each month of data taking, (b) as a function of vertex position. 
- Coincident Hits: The signal inefficiency arising from random noise in the veto shield was estimated by overlaying shield noise data on Monte Carlo signal events. A series of 3 second samples of shield noise was taken using a special untriggered run. The calculated noise rates are presented in Section 3.5.2, The sample was then divided up into $100 \mathrm{~ns}$ slices and each slice was overlaid on every Monte Carlo signal event. For each event, the probability that the event was vetoed was estimated from the number of slices that failed the veto shield requirements. The mean signal inefficiency was estimated to be $2.2 \pm 0.4 \%$ where the error represents the systematic time dependence in the shield noise rates.

- Associated Hits: Signal events can be rejected due to the hits in the shield associated with the event, for example from the muon exiting the detector between planes. The shield's scintillator modules were incorporated into the GEANT simulation of the detector, of the selected signal events, $0.31 \pm 0.07 \%$ deposited a significant amount of energy in the veto shield and would have been rejected.

The combined signal inefficiency is $2.5 \pm 0.4 \%$, where the error is dominated by the time dependence of the shield noise.

\subsubsection{Using the Veto Shield}

Applying the veto shield cuts to the data removes 51 of the 88 selected events. Using the background rejection efficiency quoted in the previous section the remaining background is estimated from data to be $1.5 \pm 0.2$ (stat.) \pm 0.2 (sys.), where the systematic error is dominated by the error in the estimate of the shield efficiency. This should be compared to the background of $1.8 \pm 0.2 \pm 0.3$ events predicted by Monte Carlo, where the systematic error is dominated by errors in the shield efficiencies and the overall normalisation of the Monte Carlo. The two estimates of the remaining background agree to better than one standard deviation.

Figure 6.15 shows the charge deposited in the shield in the pre-trigger window for the selected events. Also shown is the expected charge, predicted by overlaying shield noise onto signal Monte Carlo. No evidence of excess shield pulse height due to events with poor shield timing is seen. Figure 6.16 shows the distance to the nearest detector edge from the vertex for vetoed data events compared with the predicted distribution from cosmic ray muon Monte Carlo. 


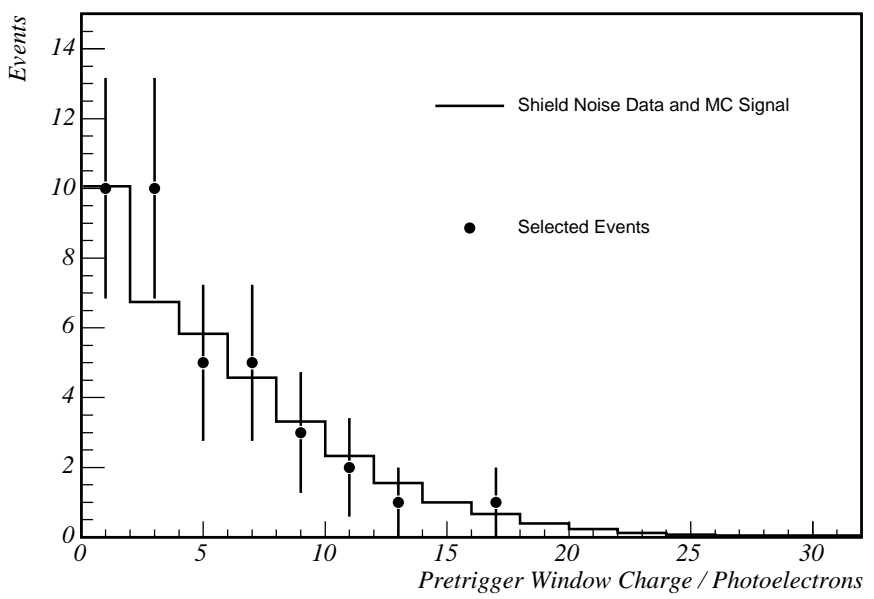

Figure 6.15: The activity in the pre-trigger time window for the selected events and a prediction using shield noise data overlaid on signal Monte Carlo.

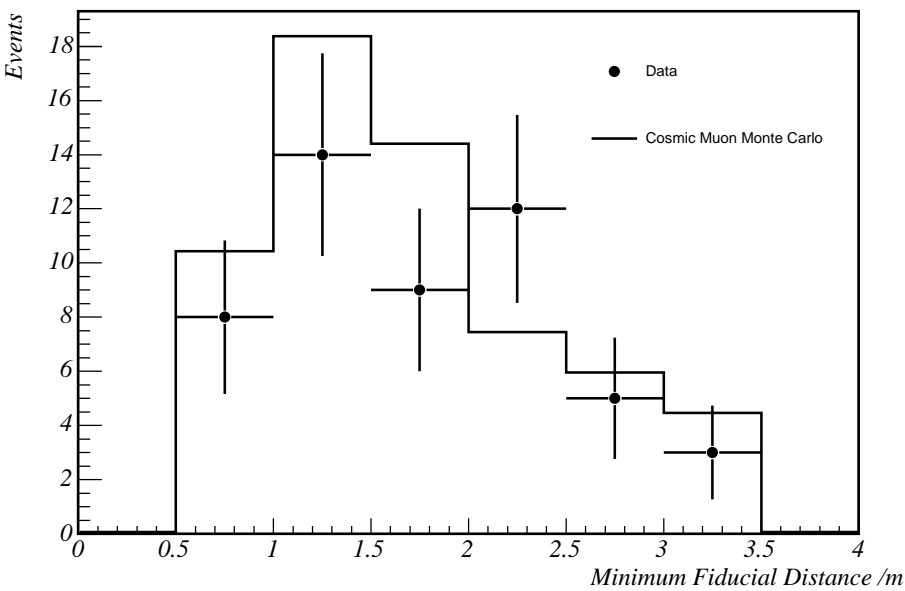

Figure 6.16: The distance to the nearest detector edge from the track's vertex (determined from timing) for vetoed events. The Monte Carlo prediction is shown by the solid line. There is a $15 \%$ uncertainty in the overall normalisation of the cosmic muon Monte Carlo. 


\subsection{Non Cosmic Muon Backgrounds}

\subsubsection{Neutron Backgrounds}

A possible source of background is muon spallation in the rock around the detector. The neutron interaction length in steel is approximately $30 \mathrm{~cm}$ so it is possible that the neutron might enter the fiducial volume before interacting. If the parent muon misses the detector then the proton track from the neutron interaction may masquerade as a contained neutrino event. Two independent methods were used to investigate neutron backgrounds.

The Soudan 2 collaboration measured the neutron background in the Soudan mine by fitting the depth distribution of selected events [29]. They observed 568 'rock' events in 7.6 years exposure, or 75 events per year above $300 \mathrm{MeV}$. If the attenuation length of neutron events and the difference in acceptance between the Soudan 2 detector and the MINOS Far Detector is taken into account, the expected number of incident neutrons above $300 \mathrm{MeV}$ in MINOS is approximately 200 events per year [91]. To estimate the acceptance of the selection cuts for neutrons, a Monte Carlo sample of isotropic incident neutrons with a uniform kinetic energy distribution between 0 and $2 \mathrm{GeV}$ was generated. For neutron energies above $300 \mathrm{MeV}$, the selection efficiency was $0.015 \pm 0.004$ (stat.)\%. Therefore the expected number of events in the data sample considered here is $0.014 \pm 0.004$. It should be noted that this estimate makes no attempt to account for the steeply falling energy spectrum of muon induced neutrons and should be regarded as an upper limit.

As a cross check, an attempt was made to select data neutron events where the neutron was accompanied by its parent muon. Events that had a through-going muon and an additional eight plane track within the fiducial volume where selected. In 83 days of data, 500 events were selected. Hand scanning showed that these events were largely dominated by multiple muons where only one of the muons was contained. When the selection cuts where applied to the contained section of the event, no events passed. It is therefore concluded that the neutron backgrounds are not significant in this analysis.

\subsubsection{Neutrino Backgrounds}

The background selection cuts are designed to reduce the dominant downward-going cosmic ray muon background. Stricter requirements are therefore placed on the upper end of the track than on the lower end. It is possible therefore, that an upward-going muon from a neutrino interaction in the rock below the detector may be selected 
by the contained event selection cuts. Upward-going muons in MINOS have been studied in detail [51]. A Monte Carlo sample corresponding to 340 years exposure was generated using the Bartol'96 flux and the contained event selection applied. The expected number of selected upward going muon events in 2.52 kton-years data in the absence of oscillations is $0.22 \pm 0.02$ (stat.) \pm 0.04 (sys.), where the systematic error is dominated by the $20 \%$ overall normalisation uncertainty.

In the presence of $\nu_{\mu} \rightarrow \nu_{\tau}$ oscillations there is an additional background from $\nu_{\tau}$ CC interactions, where the $\tau$ decays to a $\mu$. The background from $\nu_{\tau}$ interactions is estimated from Monte Carlo to be $0.45 \pm 0.06$ (stat.) \pm 0.09 (sys.) events in 2.52 kton-years, assuming $\Delta m^{2}=0.0025 \mathrm{eV}^{2}$. The systematic error is dominated by the $20 \%$ flux normalisation uncertainty, no attempt was made to include additional uncertainties from the $\nu_{\tau}$ cross section.

The background from $\nu_{e}$ interactions is also included and shown in Table 6.1.

The neutrino backgrounds vary as a function of oscillation parameters. This is accounted for when performing the oscillation analysis. 


\subsection{Signal Efficiencies}

The expected number of triggered $\nu_{\mu} \mathrm{CC}$ interactions in the MINOS far detector in 2.52 kton-years, assuming no oscillations, is $321 \pm 64$. However, only $183 \pm 37$ of these are truly contained, i.e. the muon and hadronic shower deposit no energy in the scintillator outside the fiducial volume, and of the truly contained events $91 \pm 18$ pass the DAQ triggers. The selection efficiency for truly contained triggered events as a function of neutrino energy is shown in Figure 6.17(a). The dominant loss of overall efficiency is due the requirement that the event span at least eight planes. At low energy, the selection inefficiency is low due to the short length of the muon track. The event selection efficiency switches on at a neutrino energy of approximately $600 \mathrm{MeV}$. At higher energies, i.e. greater than approximately $3 \mathrm{GeV}$, the selection efficiency starts to fall as the muon track becomes obscured by the hadronic showers. Figure 6.17(b) shows the selection efficiency as a function of the fraction of the neutrino energy carried by the produced muon. It should be noted that the selection is most efficient for events with a high muon energy fraction and therefore have a better neutrino direction resolution. Figure 6.17(c) shows the selection efficiency as a function of zenith angle for truly contained events with a true neutrino energy greater than $600 \mathrm{MeV}$. The poor acceptance, due to the minimum plane requirement, for very steep events can be clearly seen. The selection cuts are more severe on downward-going than upward-going events, reflecting the phase space occupied by the cosmic muon background, and hence the selection efficiency is asymmetric about $\cos \theta_{\text {zenith }}=0$. Figure 6.17(d) shows the selection efficiency for truly contained events as a function of the true value of $\log _{10}(L / E)$. It should be noted that the selection efficiency is quite uniform over the period of the first oscillation minimum. The slight increase in efficiency as a function of $L / E$ reflects the reduced efficiency of selecting downward-going compared to upward-going events.

\subsection{Selected Events}

A total of 37 data events is selected from 2.52 kton-years of data compared to a Monte Carlo expectation value of $29.7 \pm 5.9$ assuming maximal mixing and $\Delta m^{2}=0.0025 \mathrm{eV}^{2}$ or $40.5 \pm 8.0$ in the absence of oscillations.

It was demonstrated in the previous chapter that the event timing was not well simulated. However, it should be noted that the event selection does not use the event timing information, and will not be affected by the inaccuracies of the timing simulation. 


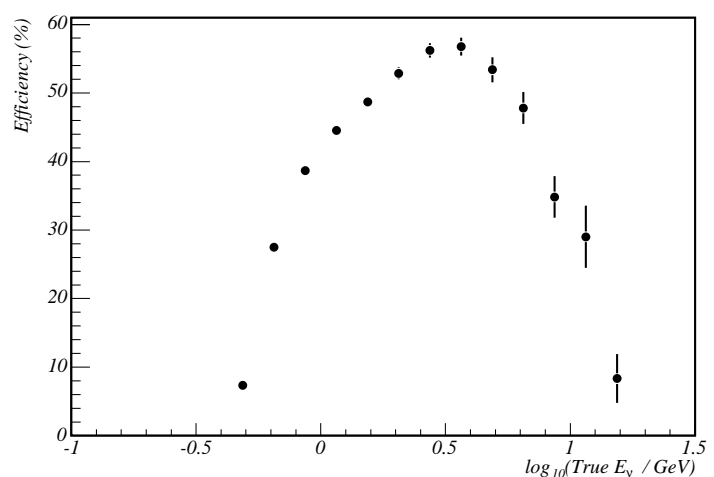

(a)

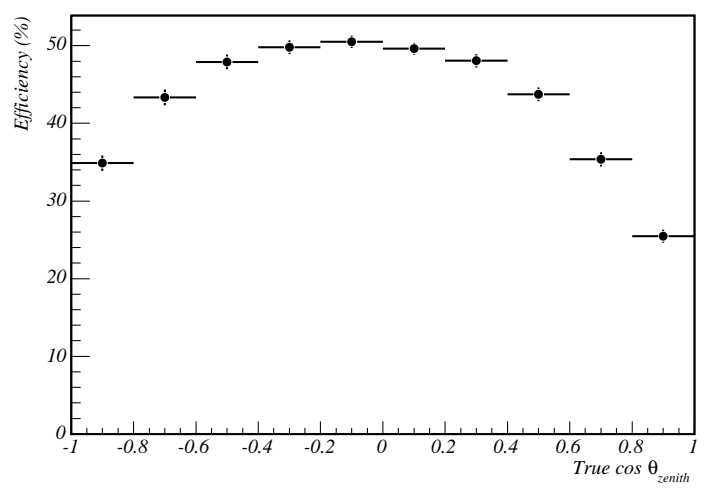

(c)

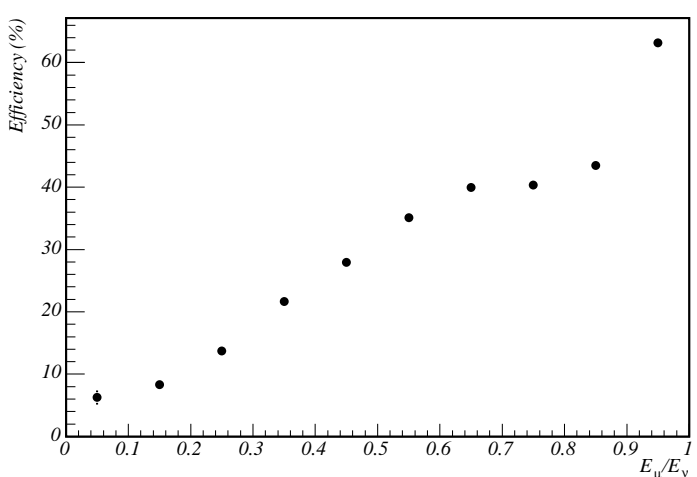

(b)

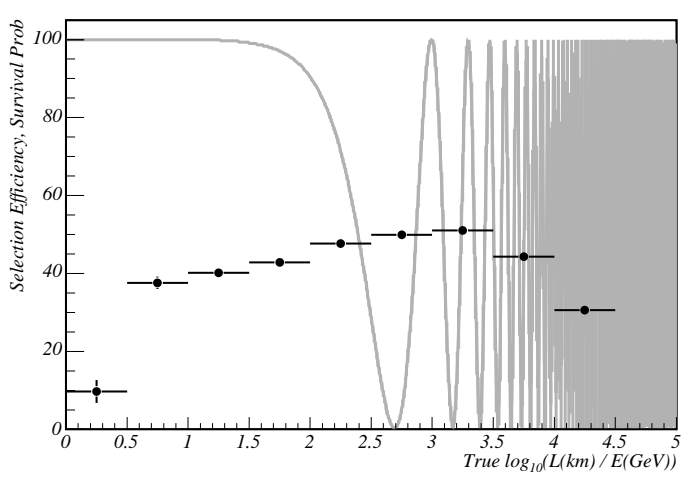

(d)

Figure 6.17: (a) The selection efficiency for truly contained $\nu_{\mu}$ CC events. (b) The selection efficiency for the same events as a function of the fraction of the neutrino energy that is imparted to the muon. (c) The efficiency for the truly contained events with an energy above $600 \mathrm{MeV}$ as a function of true neutrino zenith angle. (d) The selection efficiency as a function of true $\log _{10}(L(\mathrm{~km}) / E(\mathrm{GeV}))$ for events with $E_{\nu}^{\text {true }}>600 \mathrm{MeV}$. Superimposed onto(d) is the $\nu_{\mu}$ survival probability assuming $\left(\sin ^{2} 2 \theta=1, \Delta m^{2}=0.0025 \mathrm{eV}^{2}\right)$. 
The selected events are expected to be uniformly distributed throughout the detector. The event timing information can be used to determine the track direction. The event vertex is defined to be the early end of the track. The location of the event vertices in the $X Y$ plane is shown in Figure 6.18. Figure 6.19(a) shows the distance between the track vertex and the nearest detector edge. Figure 6.19(b) is a histogram of the nearest detector edge for the selected events. If there were still significant background contamination in the selected event sample, an excess of events near the upper edges of the detector would be observed. There is no significant evidence for excess events seen in the figures. Figure 6.19(c) shows the angle between the track direction from timing and the line joining the event to its nearest side: no excess of events pointing towards the centre of the detector is seen. Figure 6.19(d) shows the distribution of the selected events in $Z$. No evidence of an excess of events from one region in the detector can be seen.

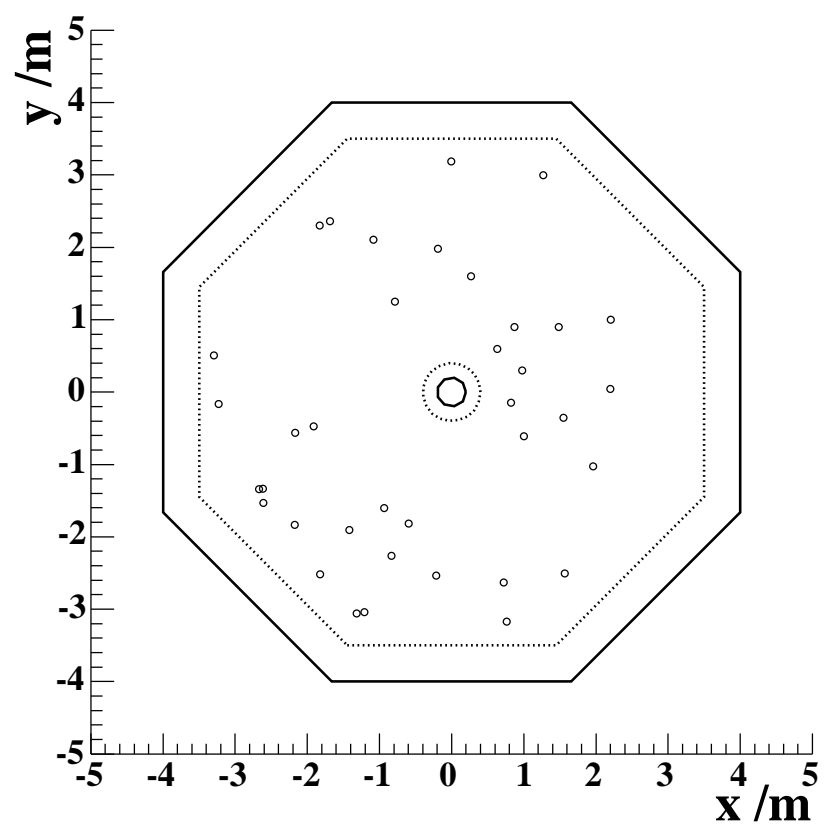

Figure 6.18: Location of the selected event vertices in the $X Y$-plane. 


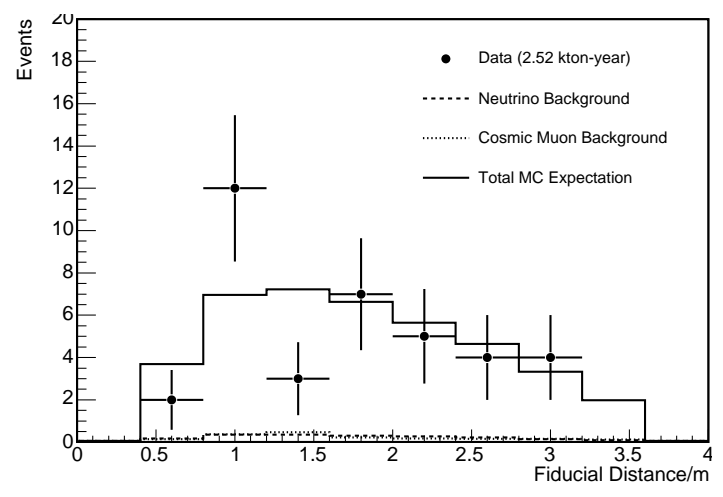

(a)

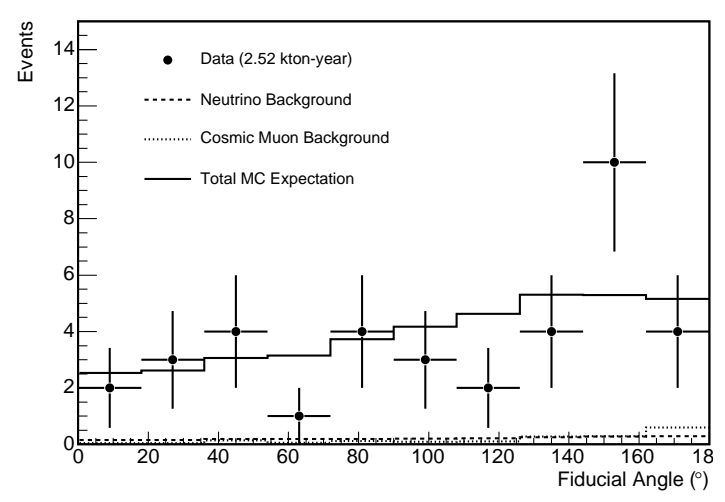

(c)

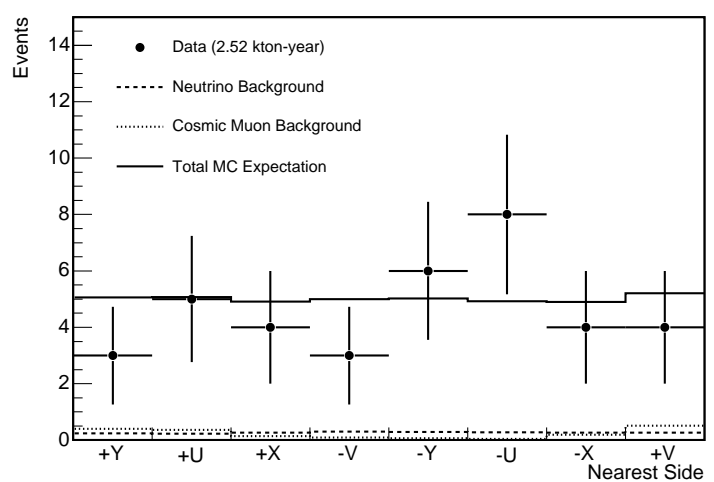

(b)

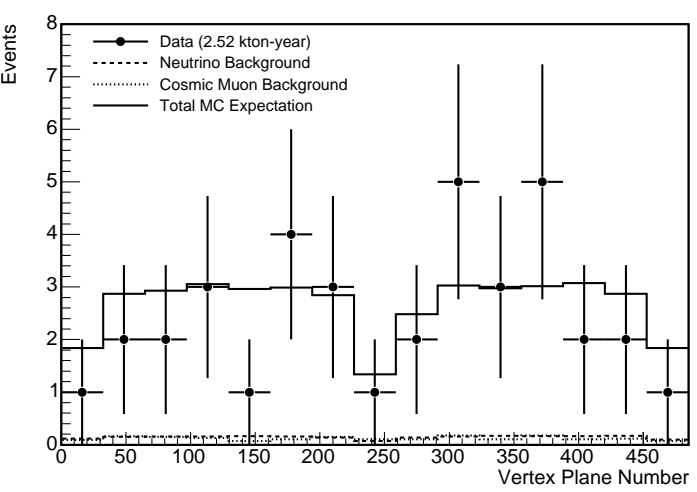

(d)

Figure 6.19: The location of the selected events in the detector. All Monte Carlo plots are normalised to the expected event rates. (a) The distance of the event vertex from the edge of the detector. (b) A histogram of the nearest edge to the event vertex. (c) The angle between the event direction and the line joining the event vertex and the nearest detector edge. (d) The distribution of the events in $Z$. 
As the data used in this analysis were taken during detector commissioning, it is important to check the temporal distribution of selected events. Figure 6.20 shows the distribution of selected events in time. To normalise within each month to the exposure, the number of selected events per stopping cosmic ray muon event is plotted. There is no indication of an excess of selected events from one period of data-taking.

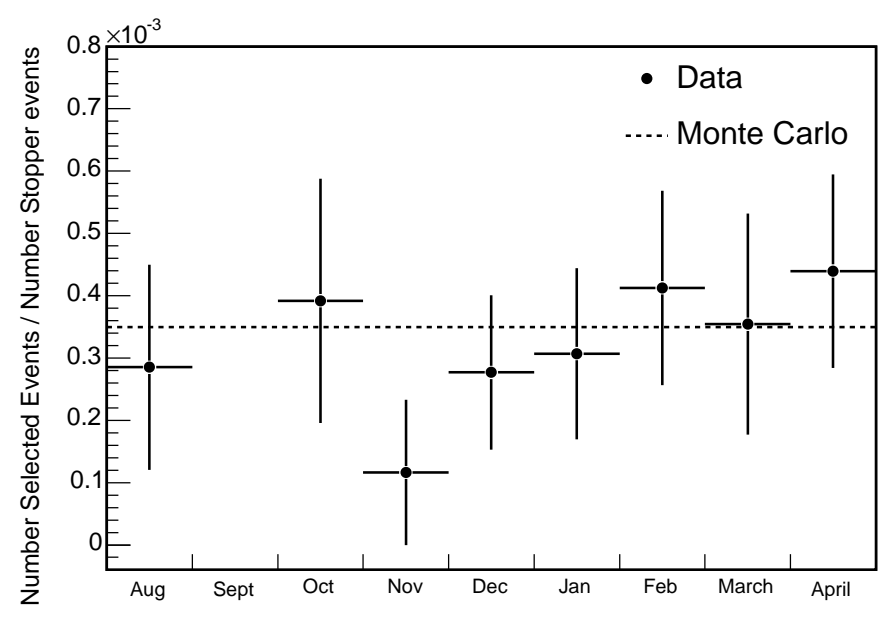

Figure 6.20: The distribution of the events in time. To account for the different exposure times month to month, the number of events in each bin has been normalised to the number of stopping cosmic ray muons observed in that bin. The dotted line shows the Monte Carlo expectation in the absence of oscillations.

Figure 6.7 shows three examples of selected events, the full set can be seen in Appendix A. Figure 6.21(a) shows a neutrino interaction where the muon and the vertex shower can be clearly identified. However, events with such a large vertex shower are less common, and most selected events have a smaller vertex shower, for example Figure 6.21(b), Figure 6.21(c) shows an example of lower energy event which are considerably more common. 

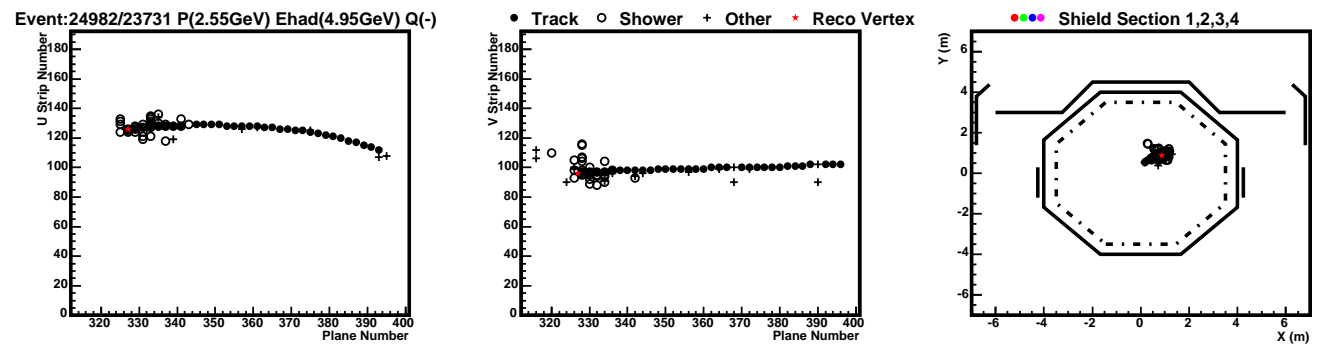

(a)
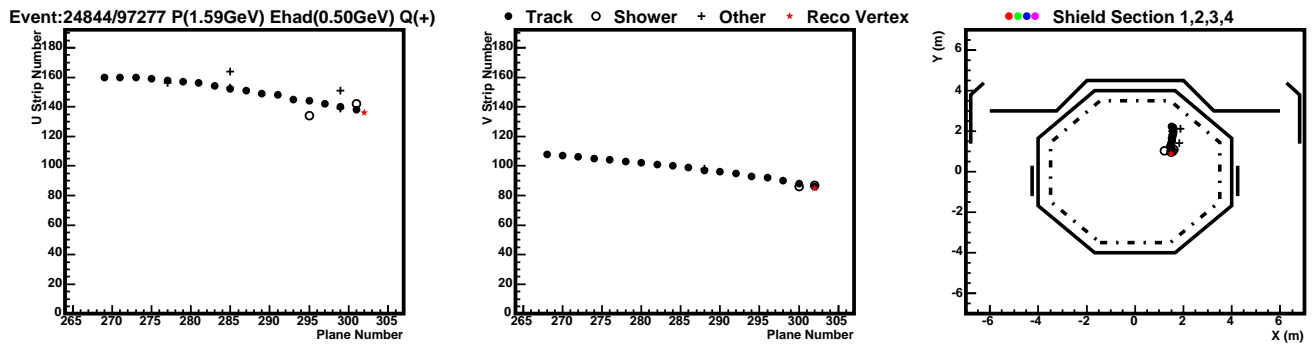

(b)
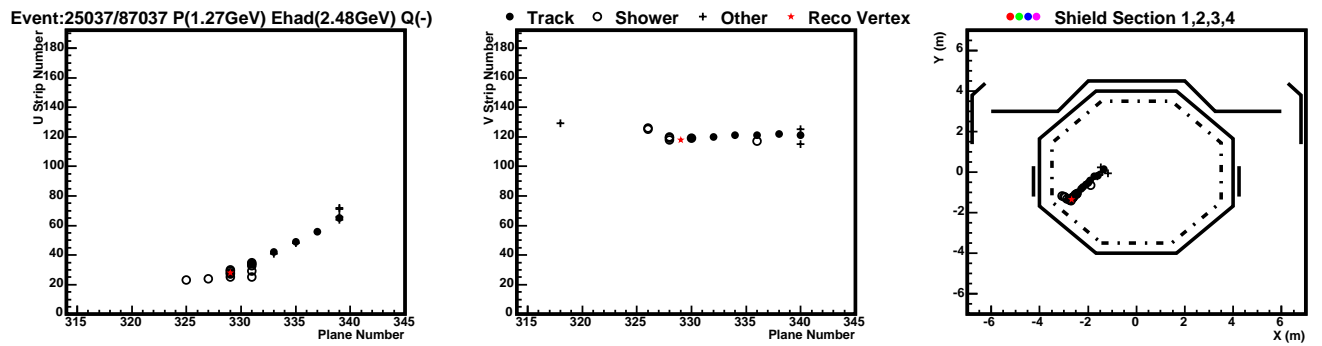

(c)

Figure 6.21: Some examples of selected events. Each plot shows the $U-Z$ and $V-Z$ views and a $3 \mathrm{D}$ reconstruction to give the $X Y$ view (right hand panel). Any in-time shield hits are shown in the $X Y$-plane colour coded according to section. Hits that were reconstructed as part of the track are shown in solid circles, shower hits are shown in open circles and unassigned hits are shown as crosses. The reconstructed vertex from timing is shown by a red star. 


\subsection{Summary}

The signal was separated from the dominant cosmic ray muon background using a series of cuts optimised using Monte Carlo. Other sources of background were investigated and estimates for their contribution to the selected event sample calculated. The final level of background contamination is less than $10 \%$. The final Monte Carlo estimate of the cosmic ray muon background agrees to within one sigma with that measured from data. There is no evidence for an additional unsimulated background present in the selected data.

\begin{tabular}{|c|c|c|}
\hline \multicolumn{2}{|c|}{ Monte Carlo } & Data \\
No oscillations & Oscillations & \\
\hline $40.5 \pm 8.0$ & $29.7 \pm 5.9$ & 37 \\
\hline
\end{tabular}

Table 6.3: Summary of selected events compared to Monte Carlo expectations in the case of no oscillations and oscillations with maximal mixing and $\Delta m^{2}=0.0025 \mathrm{eV}^{2}$.

No conclusions about the observation of neutrino oscillations can be made at this stage as the number of selected events is consistent with both no oscillations and $\left(\sin ^{2} 2 \theta=1, \Delta m_{23}^{2}=0.0025 \mathrm{eV}^{2}\right)$. The extraction of oscillation parameters is discussed in the following chapter. 


\section{Chapter 7}

\section{Neutrino Oscillation Analysis}

The direction, energy and charge of the selected events are estimated using methods developed using Monte Carlo simulations. Before a charge separated atmospheric neutrino oscillation analysis is performed, the selected events are analysed as a whole, independent of charge. The observed zenith angle and energy distributions are compared to Monte Carlo predictions using a maximum likelihood method to estimate the neutrino oscillation parameters. The selected events are then divided into candidate $\nu_{\mu}$ and $\bar{\nu}_{\mu}$ events and the analysis repeated assuming the oscillation parameters for the two charges are independent.

The data set presented here is small compared to the total exposure expected after 5 years of running the MINOS Far Detector. The predicted sensitivity obtained using events selected by this analysis for an exposure equivalent to 25 kton-years of data is presented.

\subsection{Event Properties}

\subsubsection{Event Direction}

The neutrino path length $(L)$ and hence the oscillation probability is dependent on the cosine of the neutrino zenith angle, defined to be the angle between the neutrino direction and the Earth's radial vector. Conventionally, upwards going neutrinos have a negative $\cos \theta_{\text {zenith }}$. Assuming a uniform neutrino production height and a shallow detector, $L$ can be written as,

$$
L=\sqrt{R_{\oplus}^{2} \cos ^{2} \theta_{z e n i t h}+2 R_{\oplus} h+h^{2}}-R_{\oplus} \cos \theta_{z e n i t h},
$$


where $R_{\oplus}$ is the radius of the earth $(6379 \mathrm{~km}), \cos \theta_{\text {zenith }}$ is the cosine of the neutrino zenith angle and $h$ is the neutrino production height. Therefore, the zenith angle distribution contains information about the neutrino oscillation parameters.

To reconstruct the neutrino direction, the direction of muon propagation along the track must be determined. The direction of the track can be determined by the timing of hits along its length. Two fits to the timing of hits along the track length are made to lines of gradient $\pm c$ (alternativley north-going or south-going). The fit with the least $\chi^{2}$ per degree of freedom $\left(\chi^{2} / n d f\right)$ is taken as the track direction. Once the track direction has been determined, the event vertex is identified as the starting point of the track.

For shorter tracks, the ability to determine the direction of muon propagation is degraded. From Monte Carlo it is estimated that only $92 \%$ of selected events are correctly reconstructed. Events that have ambiguous direction reconstruction are identified by cutting on the difference between the value of $\chi^{2} / n d f$ for each direction fit $\left(\left|\Delta \chi^{2}\right| / n d f=\left|\chi_{\text {north }}^{2}-\chi_{\text {south }}^{2}\right| / n d f\right)$. Figure 7.1 shows $\left|\Delta \chi^{2}\right| / n d f$ for selected $\nu_{\mu}$ charge current events. Events with small $\left|\Delta \chi^{2}\right| / n d f$ are placed in a special 'Low Resolution' sample. The position of the cut is tuned to optimise the direction reconstruction efficiency, defined as the fraction of correctly reconstructed tracks retained, and the purity, defined as the fraction of retained events that are reconstructed in the correct direction. Figure 7.2 shows the direction reconstruction efficiency and purity for a series of cuts in $\left|\Delta \chi^{2}\right| / n d f$. The cut was made at $\left|\Delta \chi^{2}\right| / n d f=0.2$, resulting in an efficiency of $81 \pm 0.5$ (stat.)\% and purity of $98.2 \pm 0.2$ (stat.)\%. Table 7.1 shows the number of events that pass the timing cut and the expectation from Monte Carlo with and without oscillations.

\begin{tabular}{|l|c|c|c|}
\hline Selection & Data & $\begin{array}{c}\text { Total Expected } \\
\text { no oscillations }\end{array}$ & $\begin{array}{c}\text { Total Expected } \\
\Delta m_{23}^{2}=0.0025 \mathrm{eV}^{2}\end{array}$ \\
\hline Good Timing & 25 & $30 \pm 6$ & $22 \pm 4$ \\
Low Resolution & 12 & $11 \pm 2$ & $8 \pm 2$ \\
\hline Total & 37 & $41 \pm 8$ & $30 \pm 6$ \\
\hline
\end{tabular}

Table 7.1: Separation of selected events into events where the direction from timing is unambiguous ('Good Timing') and events where the direction is uncertain ('Low Resolution'). The systematic uncertainty due to normalisation is shown.

It was shown in Section 5.2, that there was a significant discrepancy between event timing in data and Monte Carlo. A correction was applied to the timing of Monte Carlo events by smearing the values of $\chi^{2} / n d f$ for each timing direction fit 


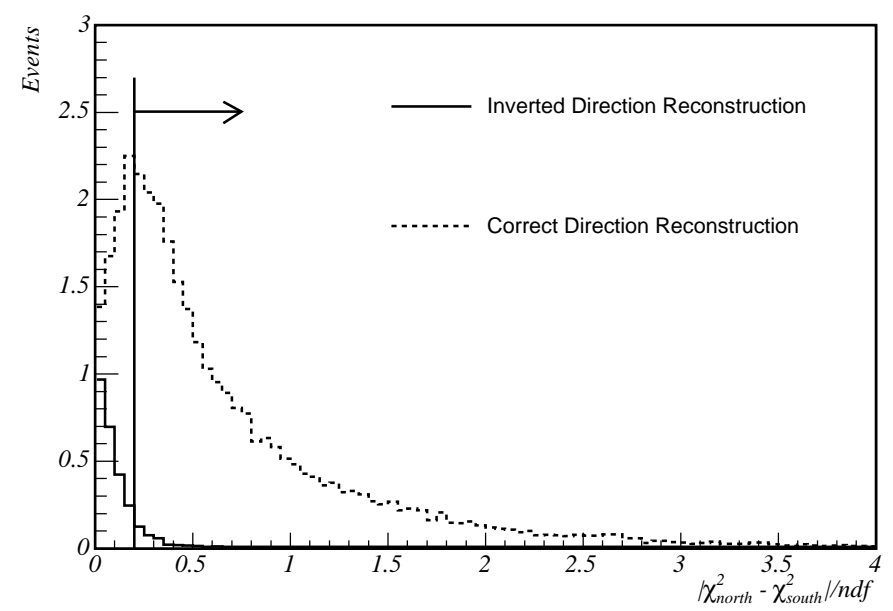

Figure 7.1: The difference in $\chi^{2} / n d f$ for the fits to the north and south going track hypotheses for $\nu_{\mu} \mathrm{CC}$ tracks that are reconstructed in the correct direction and tracks that are inverted. The cut used to flag events with suspect direction reconstruction is shown by the vertical line.

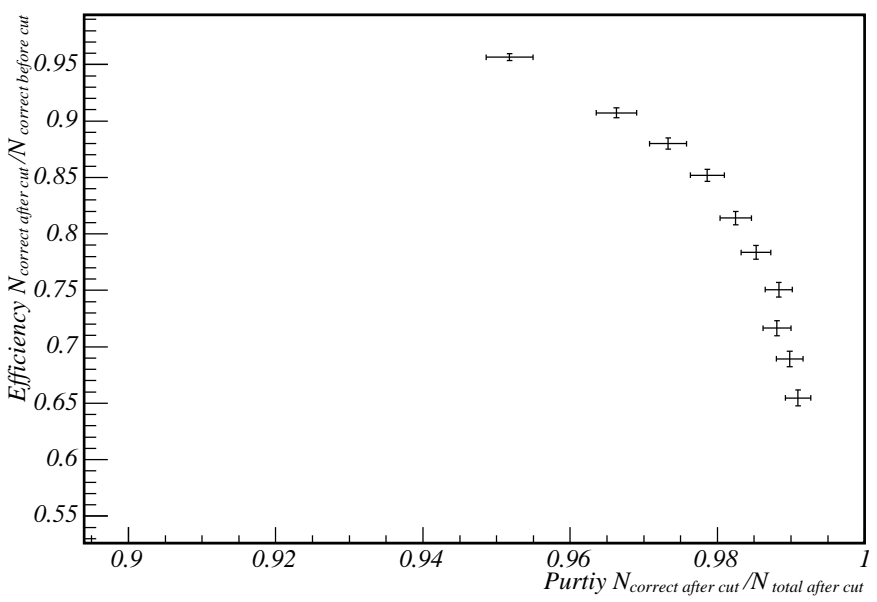

Figure 7.2: The purity, defined as the fraction of retained tracks that are reconstructed in the correct direction, against the efficiency, defined as the fraction of correctly reconstructed tracks retained by the cut for several values of cuts in $\left|\Delta \chi^{2}\right| / n d f$. Statistical errors only are shown. 
by sampling from a Gaussian of width 0.125 centred on the measure value. The track direction is then redetermined using the smeared values of $\chi^{2} / n d f$. Figure 7.3 shows the track direction reconstruction efficiency as a function of the track length for cosmic ray muons that stop in the detector both before and after the smearing was applied. The smearing gives a much improved agreement between data and Monte Carlo and is applied throughout the analysis (unless otherwise stated).

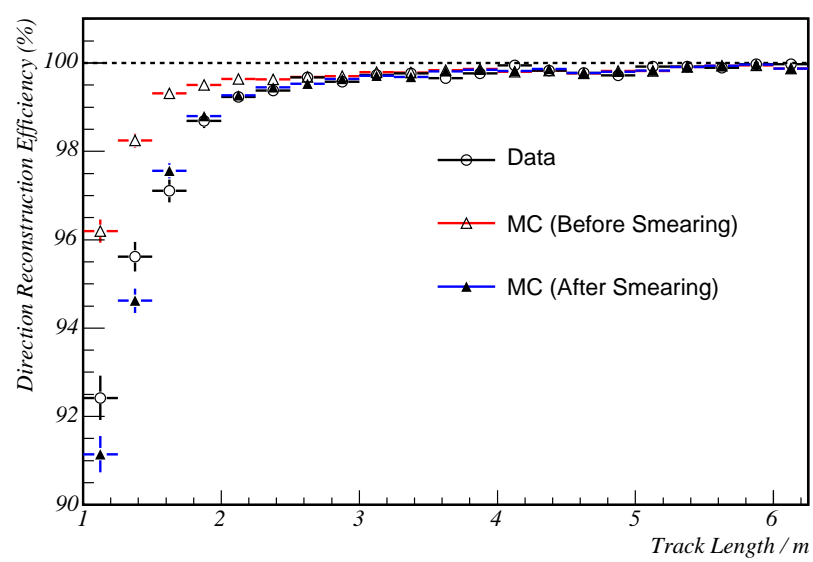

Figure 7.3: The direction reconstruction efficiency for stopping cosmic ray muons for data and Monte Carlo before and after the application of smearing to the $\chi^{2} / n d f$ value for each direction hypothesis (north and south).

Once the vertex has been identified, the muon direction is estimated by a linear fit to the track position in the first five planes of the event. Figure 7.4(a) shows the muon $\cos \theta_{\text {zenith }}$ resolution for three different ranges of true muon zenith angle. Figure 7.4(b) shows the bias and RMS of the zenith angle resolution as a function of true muon zenith angle. A clear bias as a function of zenith angle can be seen. This is due to a number of effects: the tracking software is not able to reconstruct tracks that are sufficiently deflected that they change their direction of travel in $Z$. In addition, the selection cuts have been developed to remove this class of event (see Section 6.3.4). These effects, combined with the fact that the tracking software estimates the track direction by a linear fit to the first five planes, systematically flattens steep tracks, creating a bias towards smaller values of $\left|\cos \theta_{\text {zenith }}\right|$. Figure 7.4(b) shows that the $\cos \theta_{\text {zenith }}$ resolution is significatly worse for horizontal tracks than for very steep tracks, due to the large value of $d\left(\cos \theta_{z e n i t h}\right) / d \theta$ in this region.

The neutrino direction is taken to be the direction of the muon track. Figure 7.5 


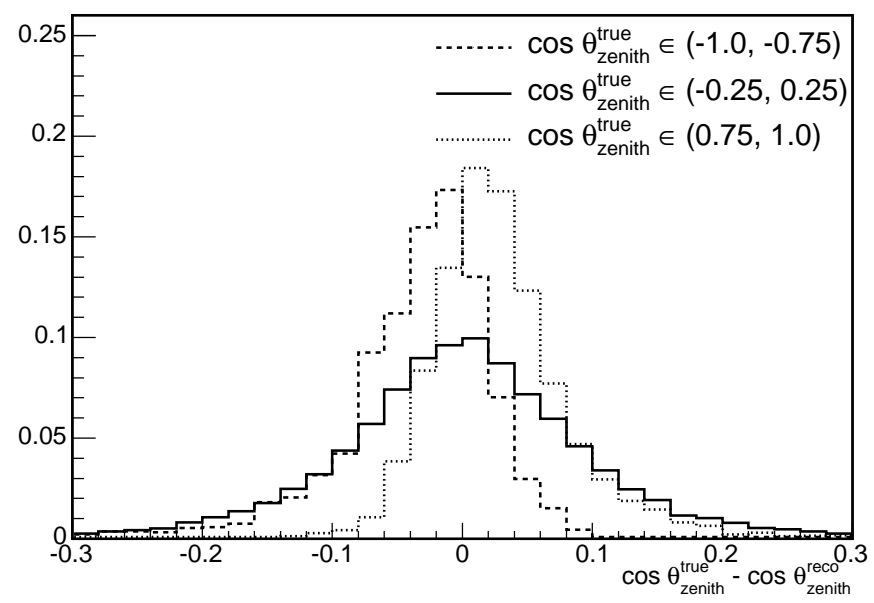

(a)

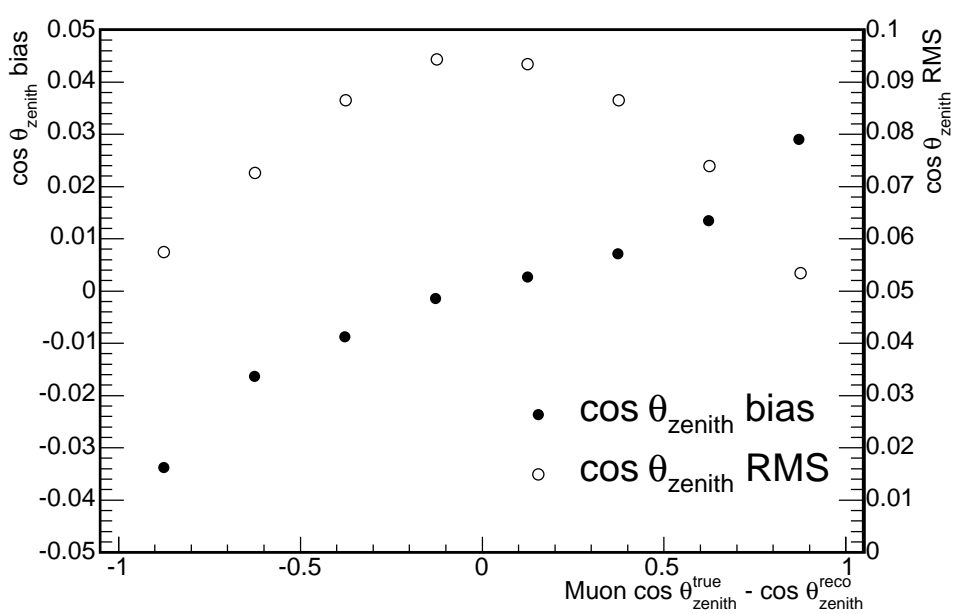

(b)

Figure 7.4: (a) The muon $\cos \theta_{\text {zenith }}$ reconstruction resolution for three different ranges of true muon zenith angle. The histograms are normalised to the same area. (b) The $\cos \theta_{z e n i t h}$ bias and RMS as a function of true muon zenith angle. 
shows the $\cos \theta_{\text {zenith }}$ resolution for selected $\nu_{\mu}$ charged current events which pass the timing cut. The solid line shows the $\cos \theta_{\text {zenith }}$ resolution for muon direction reconstruction with a RMS of 0.09 and the dashed line shows the neutrino $\cos \theta_{\text {zenith }}$ resolution with a RMS of 0.29 . Figure 7.5 demonstrates that the neutrino $\cos \theta_{\text {zenith }}$ resolution is dominated by the opening angle between the neutrino and the muon and not by the reconstruction of the muon direction. Attempts to improve the neutrino zenith angle reconstruction using the direction of the vertex shower have so far not provided any significant improvement in neutrino direction resolution due to coarse granularity of the detector.

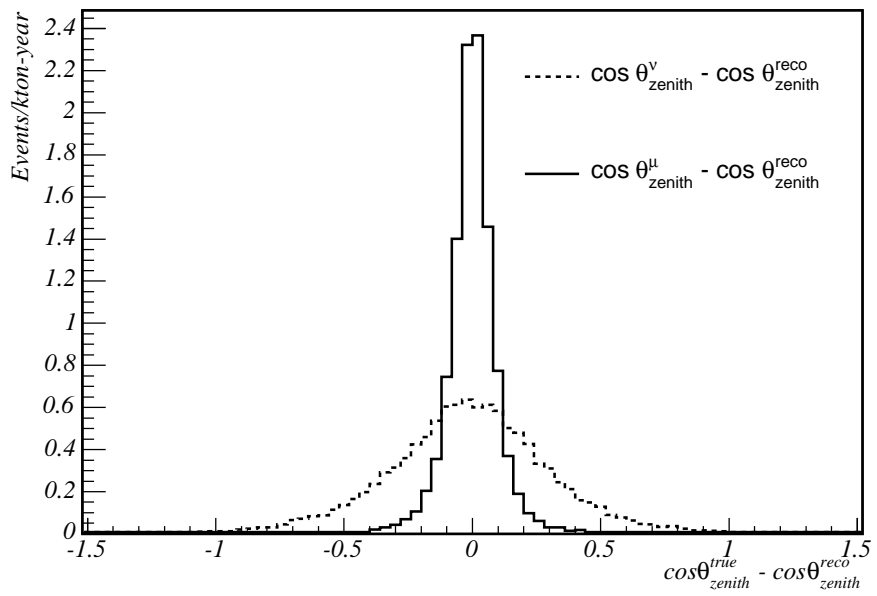

Figure 7.5: The $\cos \theta_{z e n i t h}$ resolution for selected Monte Carlo signal events that pass the timing quality cut. The muon direction resolution is shown by the solid line and the neutrino direction resolution obtained by assuming that the neutrino is collinear with the reconstructed track is shown by the dashed line.

The data $\cos \theta_{\text {zenith }}$ angle distributions are shown in Figure 7.6 for events with good timing and for the 'Low Resolution' events. The total expectation from Monte Carlo in the absence of oscillations is shown by the solid line and by the case for maximal mixing and $\Delta m_{23}^{2}=0.0025 \mathrm{eV}^{2}$ by the dot-dashed line. 


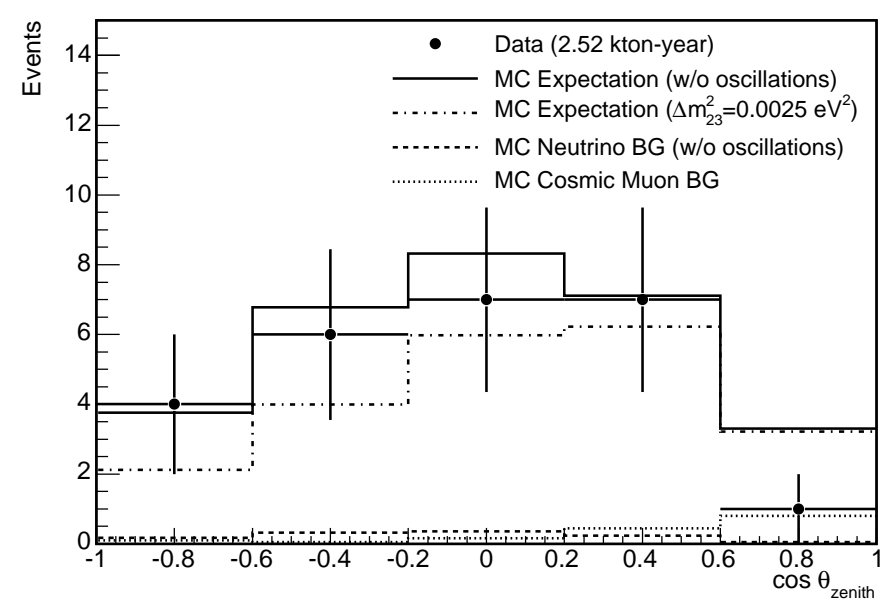

(a)

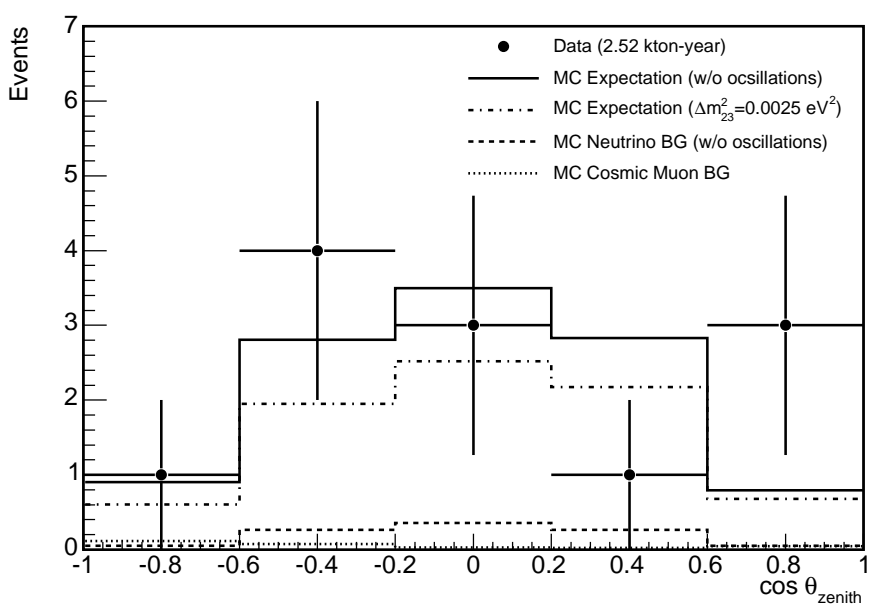

(b)

Figure 7.6: The measured and expected zenith angle distributions for (a) events with good timing and (b) 'Low Resolution' events where the direction from timing is ambiguous. The selected data events are shown by the points. The solid and dotdashed lines show the expected zenith angle distributions from Monte Carlo for an unoscillated sample and maximal mixing oscillations with $\Delta m_{23}^{2}=0.0025 \mathrm{eV}^{2}$. The expected background from cosmic muons is shown by the dashed histogram. The $20 \%$ normalisation error on the expected signal and background distributions is not shown. 


\subsubsection{Energy Reconstruction}

The energy of the selected events contains information about the oscillation parameters. To reconstruct the energy of the neutrino, the energy of the muon and the hadronic shower must be estimated.

By definition, contained events have a muon track that stops inside the fiducial volume of the detector. Therefore, the muon momentum can be determined by comparing its range through the iron and scintillator in the detector with the integral of the Bethe-Block equation [6]. The energy loss of a minimum ionising muon in one plane at normal incidence is $45 \mathrm{MeV}$. Figure 7.7 shows the fractional momentum resolution as a function of true momentum. A significant contribution to the momentum resolution comes from tracking errors, mostly caused by truncation of the track by trigger inefficiencies in the electronics. For comparison, the momentum resolution for muons where the full path of the muon has been tracked is also shown. At high energies the fractional momentum error is approximately $5 \%$ and is dominated by energy loss fluctuations. At energies below $1 \mathrm{GeV}$ the uncertainty in the track end points due to coarse sampling of the detector dominates.

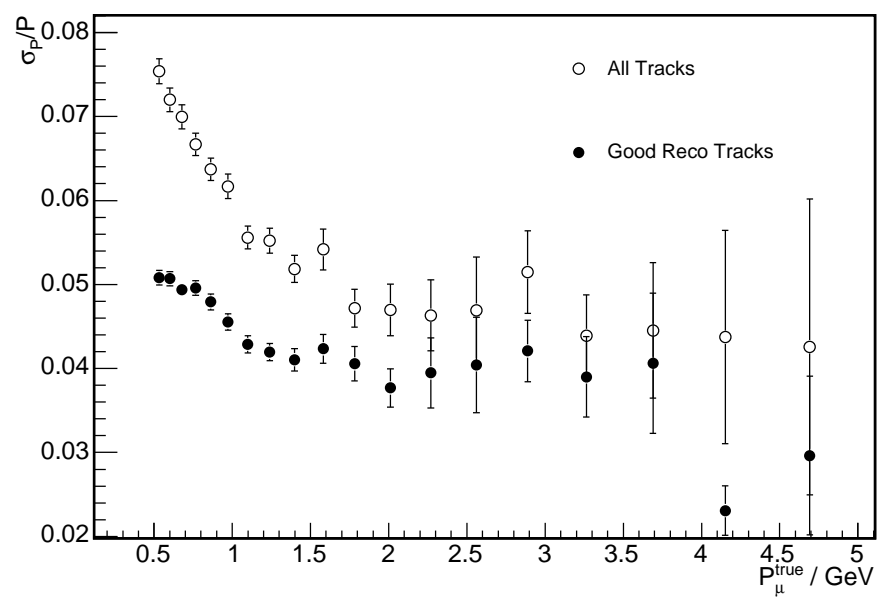

Figure 7.7: The fractional momentum resolution for selected $\nu_{\mu}$ charged current events as a function of true muon momentum. The resolution is shown for all events by the open circles and events where the muon is tracked for its full path length by the closed circles.

The hadronic energy is obtained by summing the energy of the hits which are not part of the track. An absolute energy scale is obtained from Monte Carlo which 
has been shown to give a good description of detector response to single pions and protons at normal incidence produced in a test beam [92. These studies showed that a hadronic energy resolution of $\sigma / E \sim 0.55 / \sqrt{E / \mathrm{GeV}}$ was achievable. The hadronic energy deposited (measured in photoelectrons and corrected for attenuation in the optical fibre) is compared to $\left(E_{\nu}-E_{\mu}\right)_{\text {true }}$ for the selected Monte Carlo events for 100 bins in $\log _{10} E$ between 0 and $10 \mathrm{GeV}$. The energy for a particular shower is then obtained by interpolation between points. Figure 7.8 shows the fractional energy resolution as a function of hadronic energy. For comparison, $55 \% / \sqrt{E}$ is also shown.

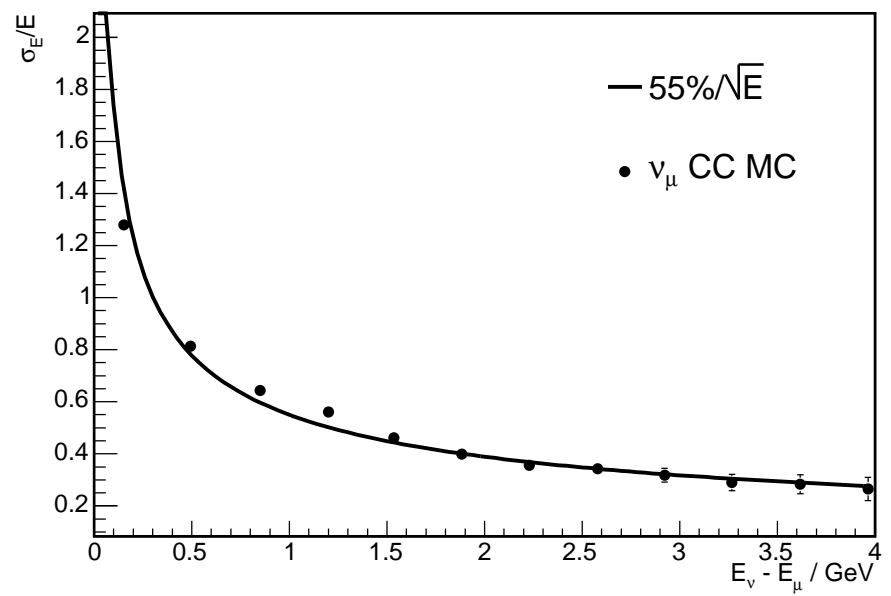

Figure 7.8: The hadronic energy resolution for selected $\nu_{\mu}$ charged current events as a function of the true $E_{\nu}-E_{\mu}$.

The energy of the neutrino is then obtained by summing the energy of the muon and the hadronic vertex shower energy. The distribution of event energies is shown in Figure 7.9, which shows good agreement with both oscillated and unoscillated expected distributions. 


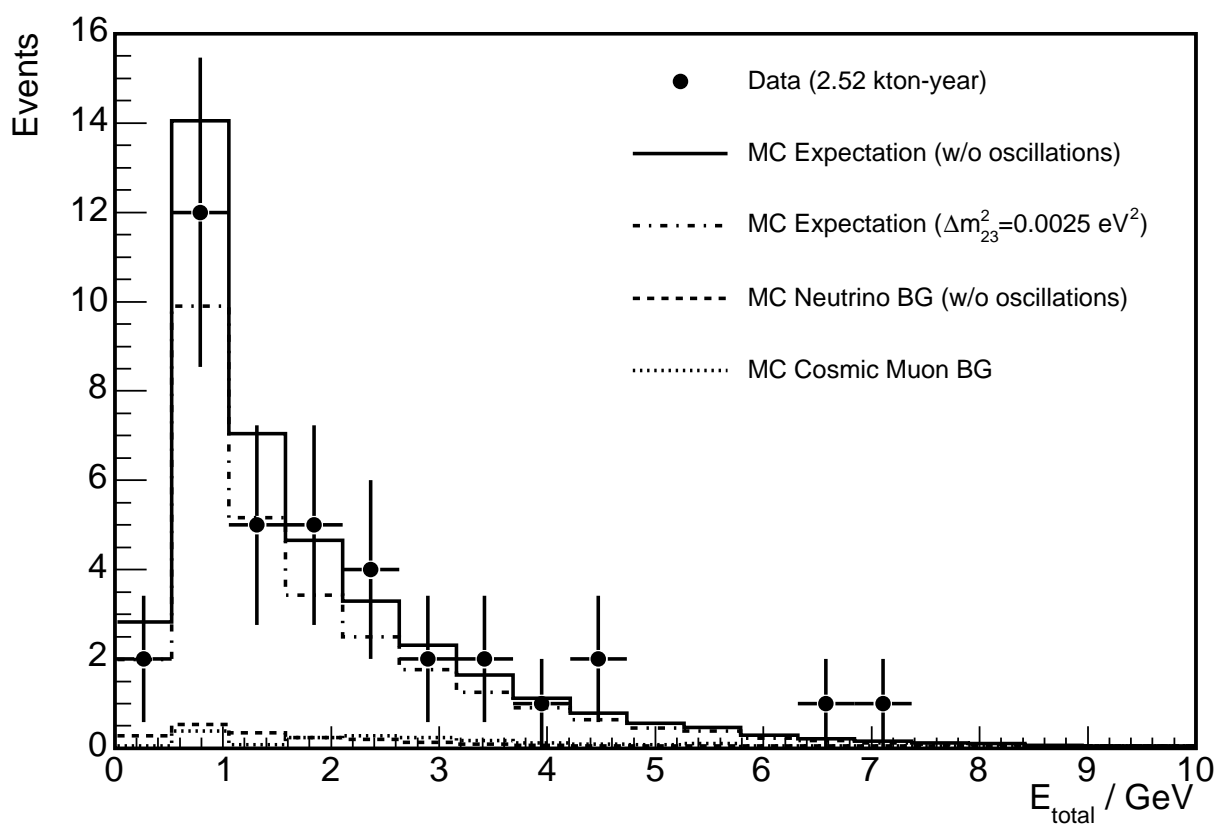

Figure 7.9: The energy of selected events obtained by summing the energies of the muon track and the hadronic system. The Monte Carlo expectation is shown for the null oscillation case and for maximum mixing with $\Delta m^{2}=0.0025 \mathrm{eV}^{2}$. The data are represented by the points. The $20 \%$ systematic uncertainty on the normalisation of the Monte Carlo is not shown. 


\subsubsection{Charge Reconstruction}

The curvature of a muon track in the magnetic field allows the charge sign of the muon to be determined. At low momentum, i.e. less than $1 \mathrm{GeV}$, the tracks are short and the charge separation is degraded. At high momentum the charge reconstruction becomes difficult due to the stiffness of the muon track. In addition, the track curvature, and thus the ability to separate positive and negative tracks, depends on the angle of the track to the magnetic field. The curvature of a track is measured and translated to the quantity $Q / P$ (track charge divided by track momentum) for multiple sections along the track. The mean value of $Q / P$ along the whole track, $\langle Q / P\rangle$ is taken as an estimator for the true curvature and the RMS of the values is taken as its error.

The reconstructed value of $\langle Q / P\rangle / \sigma_{Q / P}$ is shown for selected Monte Carlo $\nu_{\mu}$ charged current events that pass the timing cut in Figure 7.10(a). Two clear peaks can be seen corresponding to $\mu^{+}$and $\mu^{-}$. The sign of $\langle Q / P\rangle / \sigma_{Q / P}$ gives the charge of the muon and its magnitude indicates how reliable the measurement is. As indicated on Figure 7.10(a), a cut can be made on $\left|\langle Q / P\rangle / \sigma_{Q / P}\right|$ to identify events with ambiguous charge reconstruction. The position of the cut is tuned to optimise the efficiency, defined to be the fraction of tracks with correct charge identification retained by the cut, and the purity, defined to be the fraction of retained events that have been correctly identified. Figure 7.10(b) shows the effect of varying the position of the cut on the purity and efficiency of the selected sample. A cut is placed at $\left|\langle Q / P\rangle / \sigma_{Q / P}\right|>$ 0.3 , achieving a purity of $95.1 \%$ and an efficiency of $92.4 \%$. Figure 7.11 shows the purity as a function of the muon momentum for events that pass the cut. At low momentum the charge purity is degraded as the track is short. Above $1 \mathrm{GeV}$ the purity is better than $95 \%$. Figure 7.12 shows the $\langle Q / P\rangle / \sigma_{Q / P}$ distribution for selected events compared to expectation. A summary of the classification of events in shown in Table 7.2 ,

The effect of the location of the timing and charge identification cuts on the sensitivity to differences in $\Delta m^{2}$ (for neutrinos) and $\Delta \bar{m}^{2}$ (for anti-neutrinos) was investigated. Figure 7.13(a) shows the effect of varying $\Delta \bar{m}^{2}$ while fixing $\Delta m^{2}=$ $0.0025 \mathrm{eV}^{2}$ on the fraction $N_{\bar{\nu}_{\mu}} / N$, where $N$ is the total number of events selected with unambiguous charge identification and $N_{\bar{\nu}_{\mu}}$ is the number of events reconstructed with positively charged tracks. $\sigma_{\text {ratio }}$ is defined to be the size of the expected statistical error on the measured ratio $N_{\bar{\nu}_{\mu}} / N$ for the case $\Delta m^{2}=\Delta \bar{m}^{2}=0.0025 \mathrm{eV}^{2}$ and an exposure of 340 kton-years. $r$ is defined to be the difference in $N_{\bar{\nu}_{\mu}} / N$ evaluated at $\left(\Delta m^{2}=0.0025 \mathrm{eV}^{2}, \Delta \bar{m}^{2}=10^{-5} \mathrm{eV}^{2}\right)$ and $\left(\Delta m^{2}=0.0025 \mathrm{eV}^{2}, \Delta \bar{m}^{2}=0.1 \mathrm{eV}^{2}\right)$. 


\begin{tabular}{|l|c|c|c|}
\hline & Data & $\begin{array}{c}\text { Total Expected } \\
\text { no oscillations }\end{array}$ & $\begin{array}{c}\text { Total Expected } \\
\Delta m_{23}^{2}=0.0025 \mathrm{eV}^{2}\end{array}$ \\
\hline Low Resolution & 12 & $11 \pm 2$ & $8 \pm 2$ \\
\hline Ambiguous Charge & 2 & $1.5 \pm 0.3$ & $1.2 \pm 0.2$ \\
\hline Positive Charge & 6 & $9.8 \pm 2.0$ & $7.2 \pm 1.5$ \\
\hline Negative Charge & 17 & $18 \pm 4$ & $13 \pm 3$ \\
\hline
\end{tabular}

Table 7.2: Event charge classification according to timing and track curvature in the magnetic field. The 'Low Resolution' events have ambiguous direction from timing and therefore ambiguous charge. The 'Ambiguous Charge' events have good direction from timing but ambiguous charge identification.

Figure 7.13(b) shows the ratio $\sigma_{N} / r$ as a function of the position of the timing and charge identification cuts. Although no attempt was made to optimise the cuts explicitley in terms of $\sigma_{N} / r$, it can be seen from Figure 7.13(b) that the cut values used are very close to optimal. 


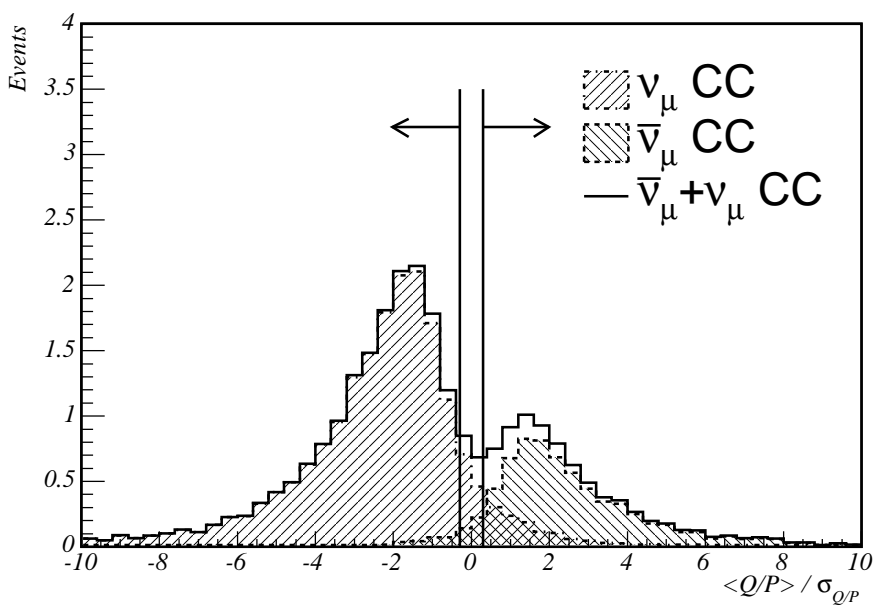

(a)

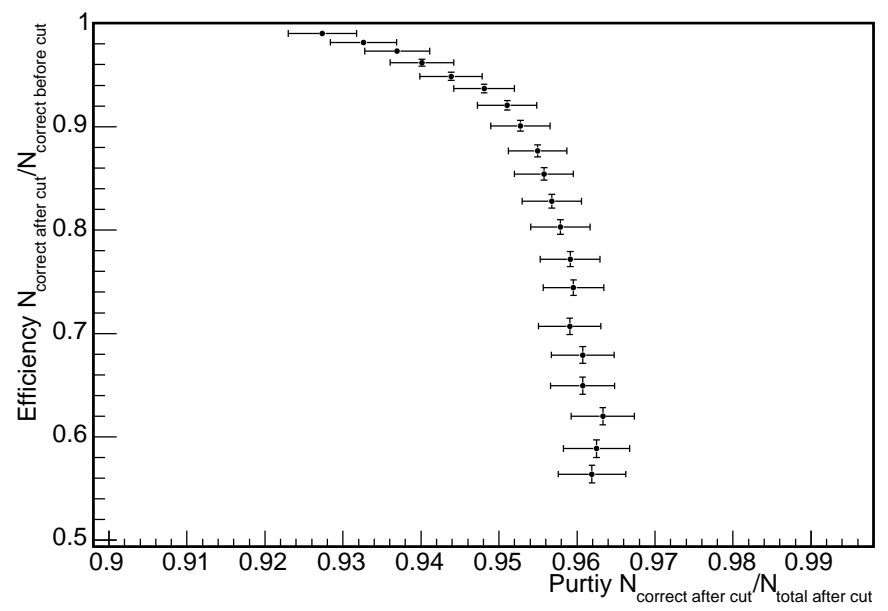

(b)

Figure 7.10: (a) The mean $Q / P$ divided by its error for the selected Monte Carlo signal events with good timing. A cut, shown by the vertical lines, is placed on $|\langle Q / P\rangle| / \sigma_{Q / P}$ to remove events with ambiguous charge. (b) The effect of the position of the cut on $\langle Q / P\rangle / \sigma_{Q / P}$ on the efficiency for retaining events which are reconstructed with the correct charge, plotted against the purity of sample retained. 


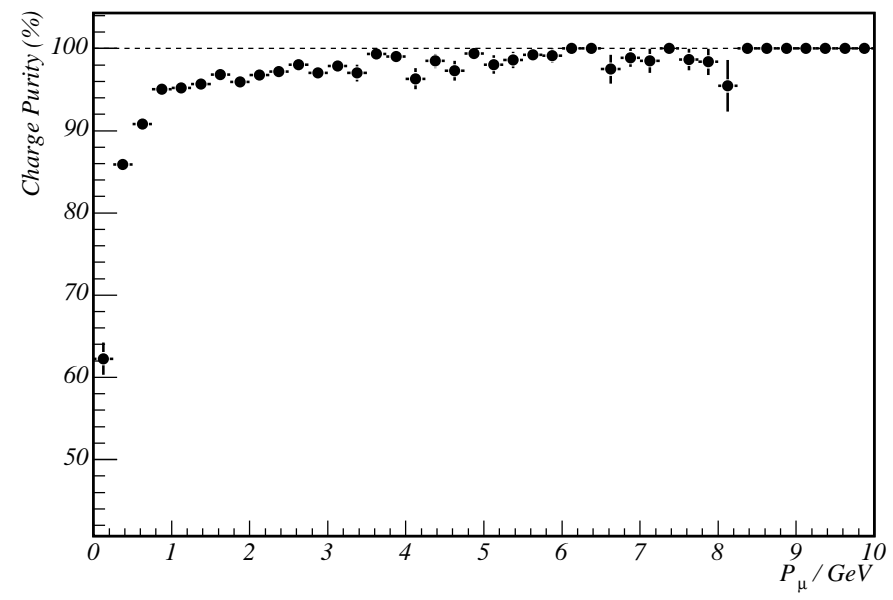

Figure 7.11: The fraction of the selected event sample after the $\langle Q / P\rangle / \sigma_{Q / P}$ cut with the charge correctly identified as a function of true muon momentum.

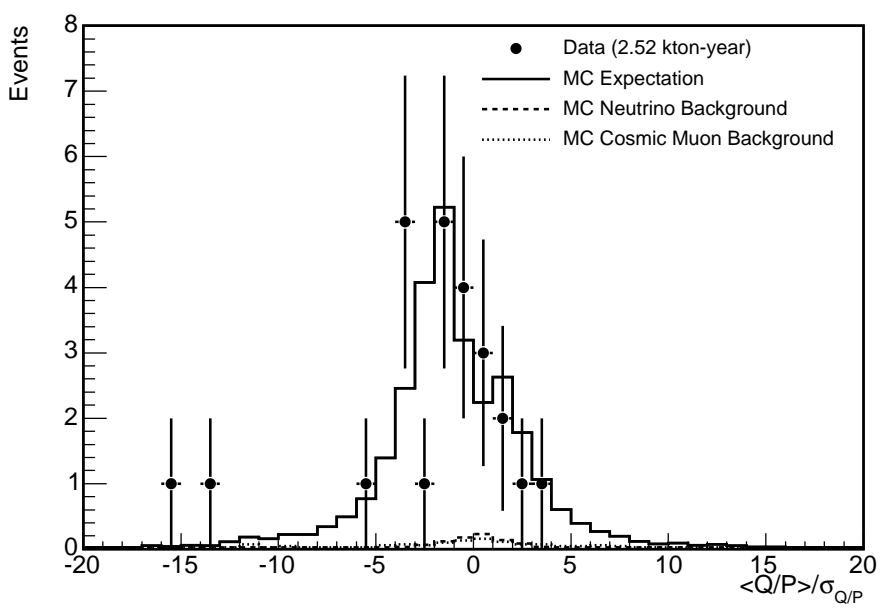

Figure 7.12: The expected $\langle Q / P\rangle / \sigma_{Q / P}$ distribution in the absence of oscillations. The observed distribution is shown by the points. 


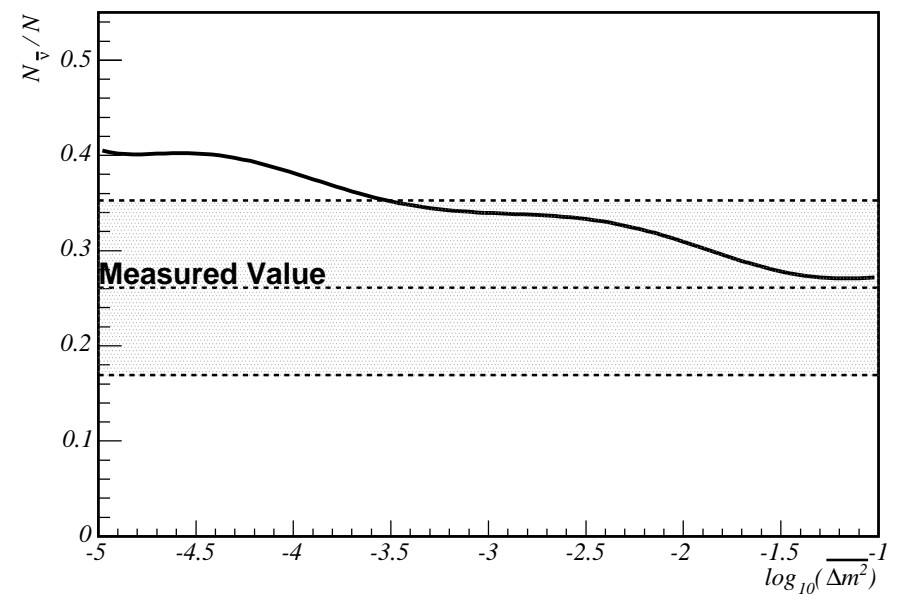

(a)

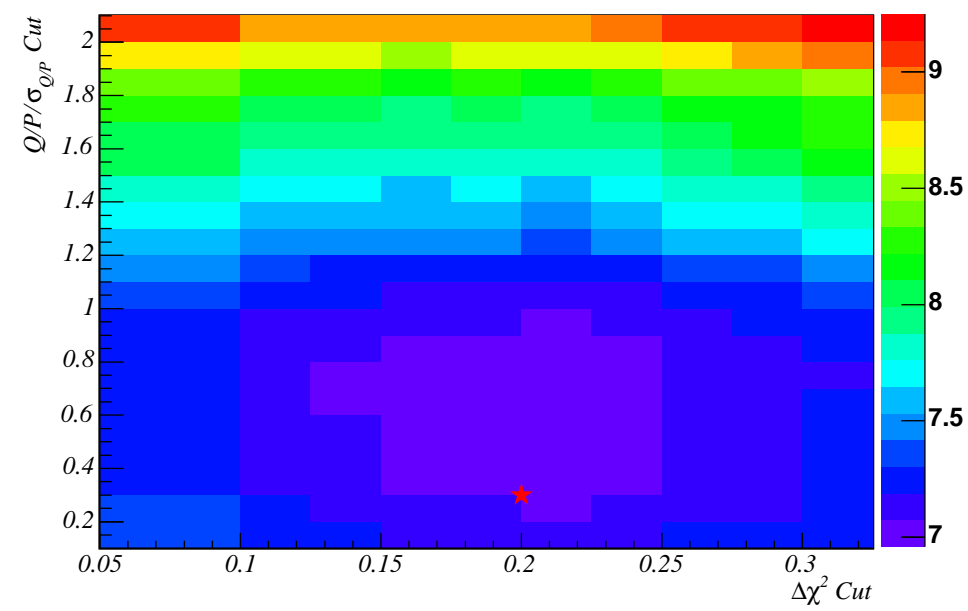

(b)

Figure 7.13: (a) The expected fraction of selected signal events with unambiguous charge identification that are reconstructed as positive as a function of $\Delta \bar{m}^{2}$ while fixing $\Delta m^{2}=0.0025 \mathrm{eV}^{2}$. The measured value is also shown, the shaded region indicate $\pm 1 \sigma$ assuming binomial errors. (b) The ratio $\sigma_{N} / r$, in arbitrary units, as a function of the position of the timing and charge identification cuts. The units on the $Z$ axis are arbitrary. The cuts values used in this analysis is shown by the star. 


\subsection{Neutrino Flux Charge Ratio}

The charge of the observed events can be used to estimate the ratio of the $\bar{\nu}_{\mu}$ and $\nu_{\mu}$ fluxes. The number of observed events of each charge can be written as,

$$
\left(\begin{array}{c}
N_{o b s}^{+} \\
N_{o b s}^{-}
\end{array}\right)=\left(\begin{array}{c}
N_{B G}^{+} \\
N_{B G}^{-}
\end{array}\right)+\left(\begin{array}{cc}
\epsilon_{\bar{\nu}} p_{\bar{\nu}}^{+} & \epsilon_{\bar{\nu}} p_{\bar{\nu}}^{-} \\
\epsilon_{\nu} p_{\nu}^{+} & \epsilon_{\nu} p_{\nu}^{-}
\end{array}\right)\left(\begin{array}{c}
\phi_{\bar{\nu}} L \sigma_{\bar{\nu}} \\
\phi_{\nu} L \sigma_{\nu}
\end{array}\right)
$$

where $\phi_{\nu(\bar{\nu})}$ is the flux of neutrinos, $L$ is the exposure and $\sigma_{\nu(\bar{\nu})}$ are the cross sections for $\nu_{\mu}\left(\bar{\nu}_{\mu}\right)$ charged current interactions. The acceptance for $\nu_{\mu}\left(\overline{\nu_{\mu}}\right) \mathrm{CC}$ events is given by $\epsilon_{\nu}\left(\epsilon_{\bar{\nu}}\right)$ and the fraction of $\nu_{\mu}\left(\bar{\nu}_{\mu}\right)$ events which are reconstructed as negative(positive) events is given by $p_{\nu(\bar{\nu})}^{-(+)} . N_{B G}$ represents the total expected background from all sources discussed in the previous chapter.

By assuming the ratio of neutrino to anti-neutrino charge current cross section is uniform over the energy range of interest (and equal to the average value in this range) and writing $\phi_{\nu}=\frac{(1-R)}{R} \phi_{\bar{\nu}}$, the above equation can be solved for $R$, the fraction of $\bar{\nu}_{\mu}$ in the atmospheric neutrino flux in the range $0.6-10 \mathrm{GeV}$. The value for $R$ obtained from data is $R=0.28 \pm 0.17$ (stat.) which agrees with the Monte Carlo expectation of 0.37 to within one standard deviation.

The systematic error introduced by the discrepancies in the timing simulations (discussed in Section 5.2 were investigated. The expected value of $R$ was then recalculated form the unsmeared Monte Carlo and a value of 0.39 obtained. The difference between the smeared and unsmeared Monte Carlo predictions of $R$ are taken as the contribution to the systematic error from the discrepancies in timing simulation, which are negligible compared to the statistical error. Therefore, the measured value of $R$ is $0.28 \pm 0.17$ (stat.) \pm 0.02 (sys.). Other sources of systematic uncertainty, for example the uncertainty on the ratio $\sigma_{\bar{\nu}_{\mu}} / \sigma_{\nu_{\mu}}$ are taken to be small compared to the statistical error. 


\subsection{Methods of Oscillation Parameter Estimation}

To measure the neutrino oscillation parameters, histograms of selected event properties are compared to the Monte Carlo expectation using a likelihood method for different input values of $\sin ^{2} 2 \theta$ and $\Delta m_{23}^{2}$. The choice of distributions is made to maximise the extraction of oscillation information. The expected likelihood surfaces for four different methods of histogramming the selected events are compared. The methods investigated are:

- The number of upward-going and downward-going events.

- The $L / E$ distribution of the selected events.

- The measured zenith angle and energy distributions.

- The measured zenith angle, energy and $E_{\mu} / E$ distributions.

For each method the average expected likelihood surface for 500 simulated experiments, each equivalent to 25 kton-years exposure, is calculated for input oscillation parameters $\sin ^{2} 2 \theta=1.0$ and $\Delta m_{23}^{2}=0.0025 \mathrm{eV}^{2}$.

To obtain the average expected likelihood surface, a set of Monte Carlo template histograms including backgrounds (discussed in the previous chapter) are generated for each point on a grid of $\sin ^{2} 2 \theta$ and $\Delta m_{23}^{2}$ and are scaled to an exposure of 25 ktonyears. Five hundred mock data sets with oscillation parameters $\left(\sin ^{2} 2 \theta=1.0\right.$, $\Delta m_{23}^{2}=0.0025 \mathrm{eV}^{2}$ ) are generated by fluctuating the Monte Carlo predicted histogram by Poisson statistics. Each mock data set is compared to every point in the parameter space and a likelihood calculated. The likelihood includes the $20 \%$ uncertainty in the overall normalisation of the neutrino flux and the $15 \%$ cosmic ray normalisation uncertainty as nuisance parameters. The likelihood for each point is averaged over the mock data sets giving the mean expected likelihood surface. To compare the relative sensitivities obtained by the different methods, Figure 7.14 shows the likelihoods along the line $\sin ^{2} 2 \theta=1.0$. The different methods are described in detail below.

\section{Up-Down}

The first method compares of the number of upward and downward-going events. The expected fraction of events that pass the timing cut and are reconstructed as upward-going is shown in Figure 7.15 . 


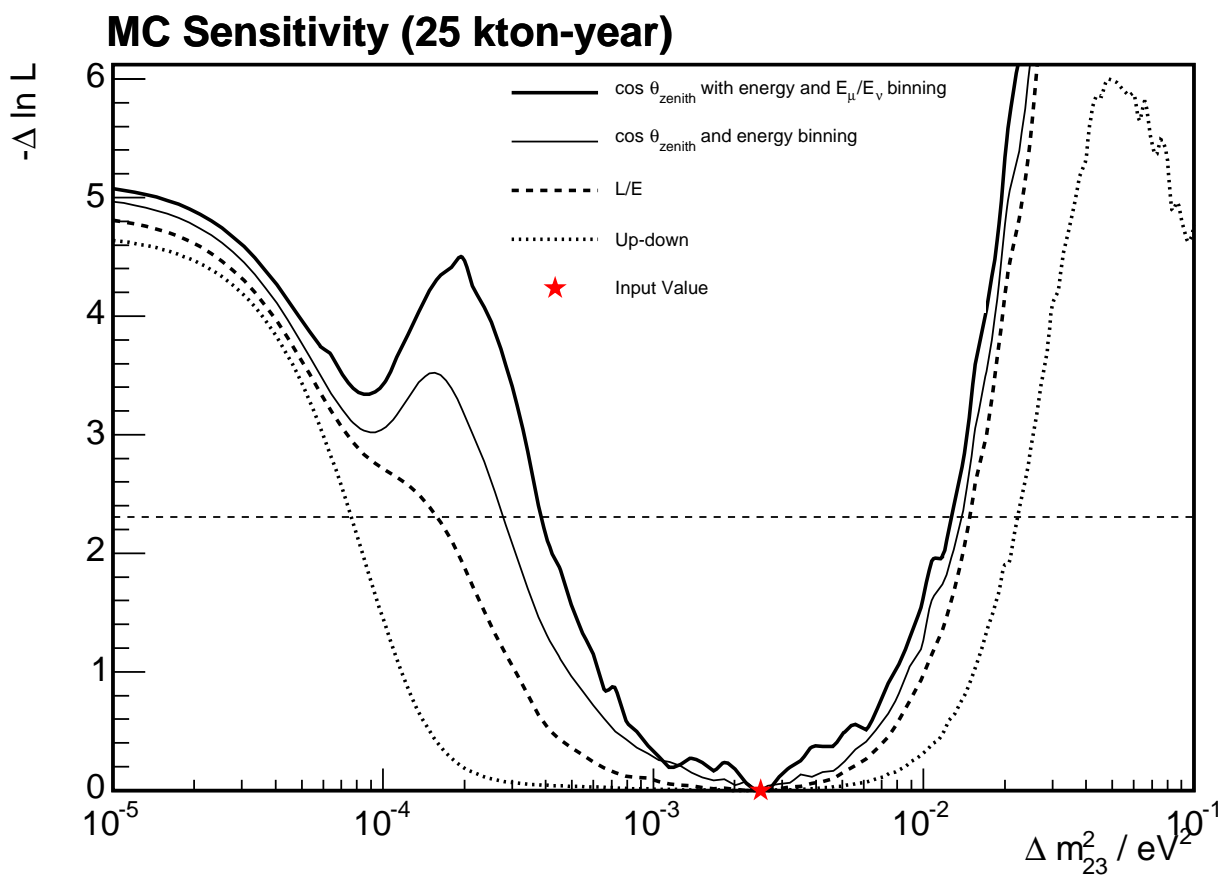

Figure 7.14: The average expected likelihood along the line $\sin ^{2} 2 \theta=1$ obtained by four different methods of fitting and a simulated exposure equivalent to 25 kton-years. The input value was $\Delta m_{23}^{2}=0.0025 \mathrm{eV}^{2}$ and maximal mixing and is shown by the star. The value of $-\Delta \ln L$ corresponding to a $90 \%$ confidence limit is shown by the dashed horizontal line.

The likelihood at each point on the $\sin ^{2} 2 \theta / \Delta m_{23}^{2}$ grid is calculated using equation 7.3 where $p\left(u ; \Delta m_{23}^{2}, \sin ^{2} 2 \theta\right)$ and $p\left(d ; \Delta m_{23}^{2}, \sin ^{2} 2 \theta\right)$ are the probabilities of observing an upward or downward going event respectively. The number of upward and downward going events observed in each mock data set are $N_{o b s}^{u}$ and $N_{o b s}^{d}$ respectively. The total number of observed and expected events are represented by $N_{o b s}$ and $N_{\text {exp }}$ and $\sigma_{\text {exp }}$ represents the systematic error on $N_{\text {exp }}$, which is taken to be $20 \%$.

$$
\begin{gathered}
-\ln L\left(\Delta m_{23}^{2}, \sin ^{2} 2 \theta\right)=-N_{o b s}^{u} \ln p\left(u ; \Delta m_{23}^{2}, \sin ^{2} 2 \theta\right)-N_{o b s}^{d} \ln p\left(d ; \Delta m_{23}^{2}, \sin ^{2} 2 \theta\right) \\
+\frac{\left(N_{o b s}-N_{e x p}\right)^{2}}{2 \sigma_{\text {exp }}^{2}}
\end{gathered}
$$

Figure 7.14 shows that the up-down method produces limits on $\Delta m_{23}^{2}$ of approximately $10^{-4}$ to $10^{-2}$, which is consistent with the flat central region seen in Figure 7.15 . 


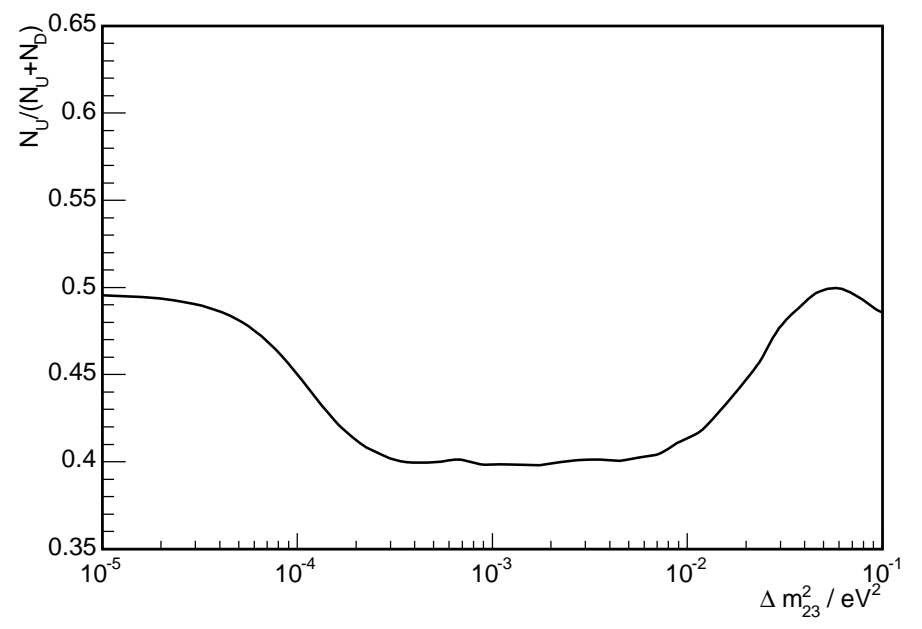

Figure 7.15: The expected fraction of upward going events that pass the timing quality cut as a function of $\Delta m_{23}^{2}$.

\section{L/E Distribution}

The second method is similar to that used by Soudan 2 [29]. Events are separated into two samples, a sample where the direction can be obtained from timing and the 'Low Resolution' sample. For each event in the good timing sample, a value for L is estimated using Equation 7.1, where the production height was assumed to be $20 \mathrm{~km}$ for all zenith angles and energies. The value $L / E$ is then constructed, using the the measured neutrino energy. Neutrino oscillations cause a reduction in the number of events compared to expectations at a specific value of $L / E$. Figure 7.16 shows the $L / E$ resolution for events with good timing. The central peak has a width of 0.25 and the long tails are due to the horizontally travelling neutrinos, where $d L / d \theta_{\text {zenith }}$ is large.

Events with good timing resolution are binned in $\log _{10}(L / E)$. The width of the bins is 0.25 , which is comparable to the experimental resolution in $L / E$. The low resolution events are binned in a single bin as they provide little shape information but do provide information about flux normalisation. Figure 7.17 shows the expected $L / E$ distributions in the absence of oscillations and with oscillations at maximal mixing and $\Delta m_{23}^{2}=0.0025 \mathrm{eV}^{2}$.

The likelihood function used is given in Equation 7.4, where $N_{i}^{\nu}$ and $N_{i}^{B G}$ are the numbers of expected events in the $i^{\text {th }}$ bin due to neutrino events and background events respectively. The normalisation uncertainties in both atmospheric neutrino 


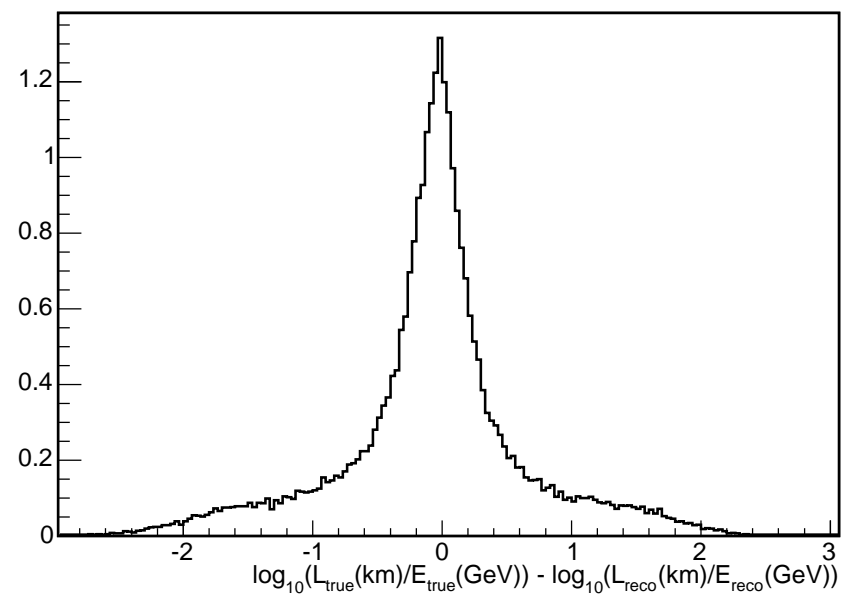

Figure 7.16: The $\log _{10}(L / E)$ resolution for events with good timing. The histogram has been normalised to unit area.
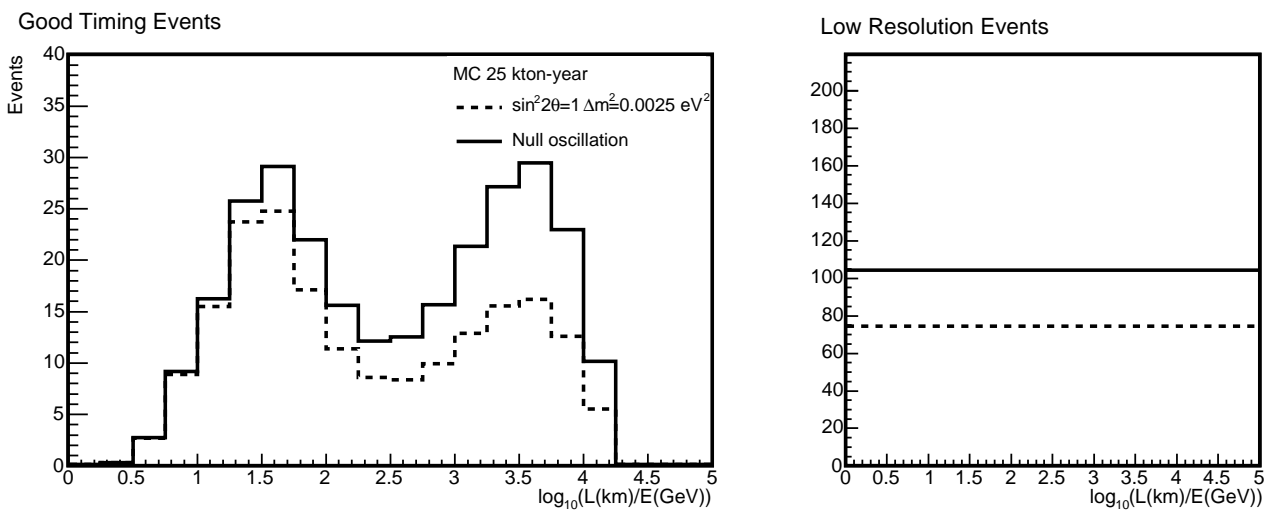

Figure 7.17: The expected $\log _{10}(L / E)$ distribution for an exposure of 25 kton-years, in the absence of oscillations (solid line) and with oscillations at maximal mixing and $\Delta m_{23}^{2}=0.0025 \mathrm{eV}^{2}$ (dashed line). The left-hand panel shows shows the distribution for events with good timing. The right hand panel shows events that fail the timing quality cut. 
flux and background cosmic ray muon flux are included as nuisance parameters $\alpha$ and $\beta$ and are given a Gaussian error term in the likelihood function. The likelihood is minimised at each point in parameter space with respect to $\alpha$ and $\beta$.

$$
\begin{gathered}
-\ln L=\sum_{\text {bins }(i)}\left(\alpha N_{i}^{\nu}+\beta N_{i}^{B G}+\ln \left(N_{i}^{\text {data }} !\right)-N_{i}^{\text {obs }} \ln \left(\alpha N_{i}^{\nu}+\beta N_{i}^{B G}\right)\right) \\
+\frac{(1-\alpha)^{2}}{\sigma_{\alpha}^{2}}+\frac{(1-\beta)^{2}}{\sigma_{\beta}^{2}}
\end{gathered}
$$

Figure 7.14 shows that only modest gains are made in terms of confidence limits by using a $L / E$ style parameter fitting over the simple up-down ratio. Most notably there is a reduction in the lower limit of $\Delta m_{23}^{2}$ due the partial resolution of the first oscillation minimum driven by the relative height of the two peaks in the $L / E$ distribution.

The limitation of the $L / E$ method is that it does not account for the variation of $L / E$ resolution as a function of energy and zenith angle. Figure 7.18 shows the $\log _{10}(L / E)$ resolution as a function of the cosine of zenith angle and the neutrino energy. The $L / E$ resolution is particularly poor for horizontal events due the large value of $d L / d \theta_{\text {zenith }}$ in this region. It is also poor for low energy events due to the reduced Lorentz boost and the shorter muon tracks.

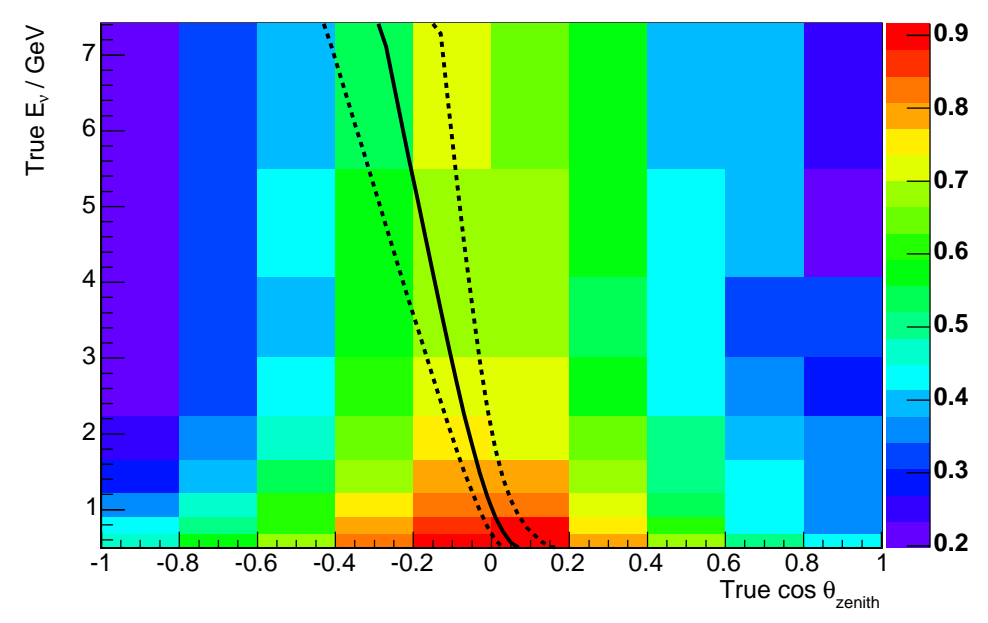

Figure 7.18: Absolute $\log _{10}(L / E)$ resolution as a function of neutrino energy and zenith angle. The first oscillation minimum assuming $\sin ^{2} 2 \theta=1$ and $\Delta m_{23}^{2}=$ $0.0025 \mathrm{eV}^{2}$ is shown by the solid line. The $50 \%$ oscillation points are shown by the dashed lines. 


\section{Zenith Angle and Energy Distributions}

By histogramming events in the cosine zenith angle and the logarithm of the reconstructed energy as shown in Figure 7.19, the events with good $L / E$ resolution are well separated from events with reduced resolution. The same likelihood function used in the $L / E$ method (Equation 7.4) can be used to determine the best fit point for each mock data set. The resulting likelihood curve is shown in Figure 7.14 by the thin continuous line. A slight improvement is seen in the lower limit of $\Delta m_{23}^{2}$ due to the improved resolution of the first minimum. A clear peak can be seen at $\Delta m_{23}^{2}=2 \times 10^{-4} \mathrm{eV}^{2}$. The width of the first oscillation minimum broadens in cosine zenith angle with decreasing $\Delta m^{2}$ until at approximately $\Delta m_{23}^{2}=1 \times 10^{-4}$ the width is approximately 1 at $1 \mathrm{GeV}$ and the experiment is no longer able to resolve the dip. At lower values of $\Delta m_{23}^{2}$ only very low energy neutrinos are oscillating and no oscillation minimum is visible. It is interesting to note that with a neutrino energy threshold of $600 \mathrm{MeV}$ the smallest $\Delta m_{23}^{2}$ resolvable by the fully contained events is of the order of $6 \times 10^{-5} \mathrm{eV}^{2}$, which is the point at which the different methods converge, indicating that for values lower than this all methods rely solely on the up-down ratio.

\section{Zenith Angle, Energy and $E_{\mu} / E$ Fraction Distributions}

As the cosine zenith angle/energy method is simple and yields reasonable results, it is the method used in this analysis. However, it is worth noting possible future improvements that can be made. The most notable is the isolation of events where the muon direction follows the neutrino direction. The muon-neutrino opening angle is governed by two kinematic variables. Firstly, the neutrino energy, the higher the neutrino energy, the greater the Lorentz boost. Secondly, the interaction $y$ (defined to be $\left.\left(E_{\nu}-E_{\mu}\right) / E_{\nu}\right)$, the larger the fraction of the neutrino energy imparted to the muon, the more collinear the muon will be with the initial neutrino direction. Figure 7.20 shows the neutrino direction resolution in degrees as a function of total reconstructed energy and the fraction of neutrino energy carried by the muon for events where a vertex shower was reconstructed.

Events with good timing were separated into four bins in $E_{\mu} / E_{\nu}, 10$ bins in zenith angle and 4 bins in energy. Using the same likelihood as the two previous methods gives the likelihood curve shown by the thick solid line in Figure 7.14. Apart from the increase in the peak at $2 \times 10^{-4}$ due to better resolution of the first oscillation minimum, there is no significant increase in sensitivity. This method was not used 

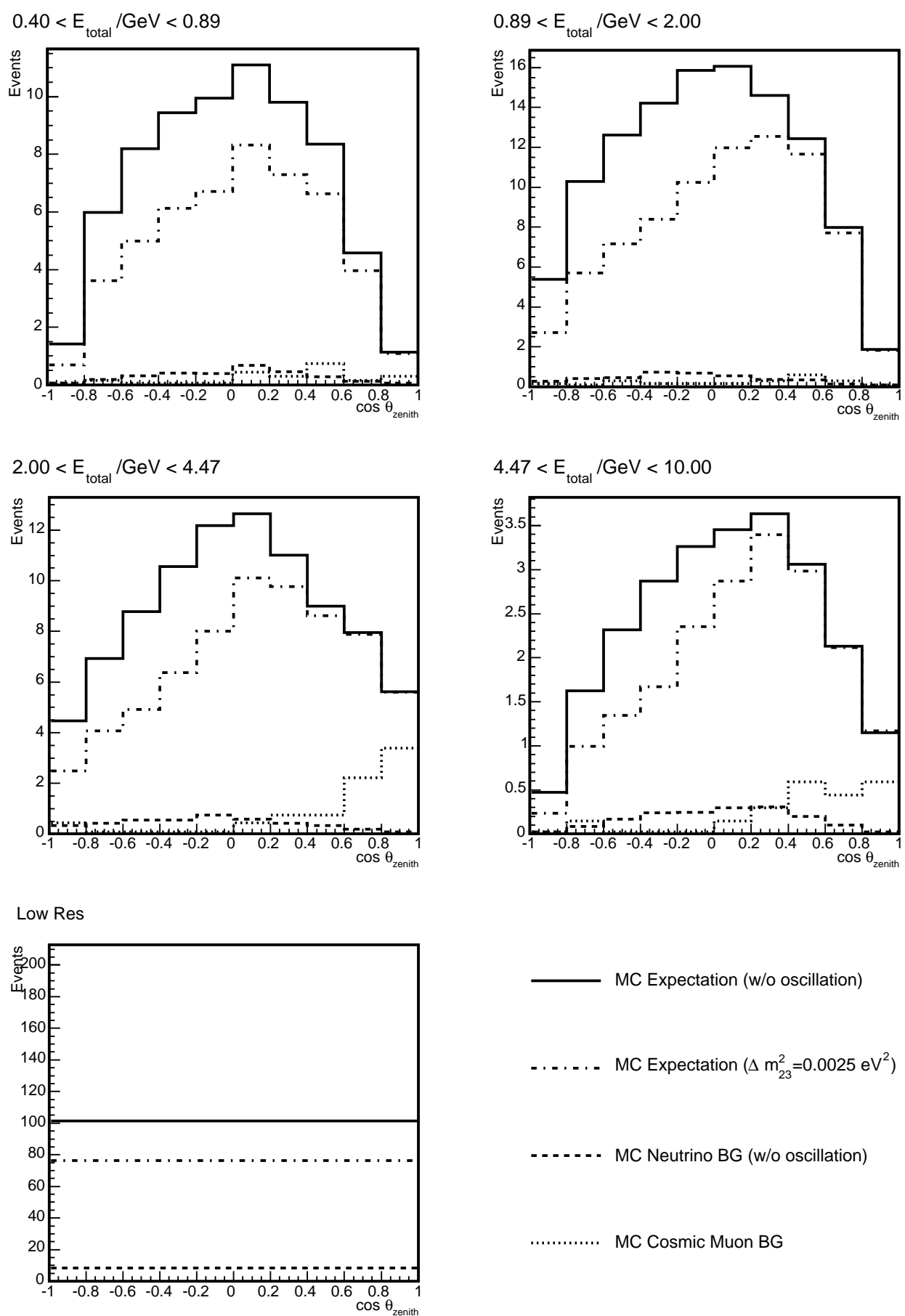

Figure 7.19: The expected zenith angle distributions for a 25 kton-years exposure. 


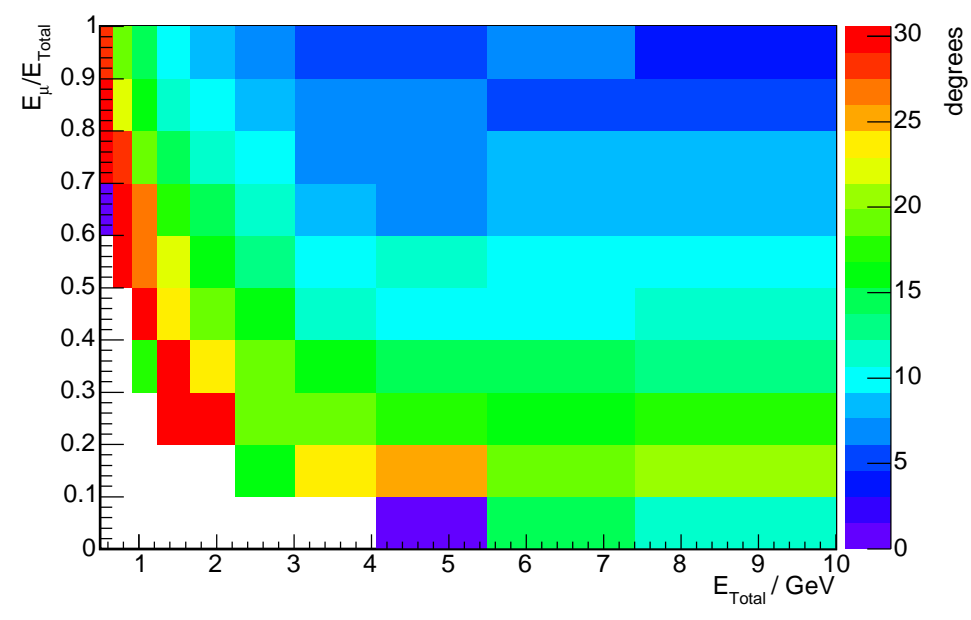

Figure 7.20: The neutrino angle resolution as a function of total reconstructed energy, $E_{\text {total }}$, and the fraction of the total reconstructed energy imparted to the muon, $E_{\mu} / E_{\text {total }}$.

in the following analysis due to its strong dependence on shower finding algorithms and Monte Carlo simulation of hadronic interactions which have yet to be studied in detail.

\subsection{Discussion of Systematics}

Various sources of systematic uncertainty are considered. Due to the small size of the data sample presented in this analysis the resolution of the oscillation parameter measurement is dominated by the statistical error. However, the effect of the systematic uncertainties on future measurements is investigated.

\subsubsection{Normalisation}

The impact of the uncertainty on the normalisation of the neutrino flux (discussed in Section 5.1) is investigated.

It may be possible to reduce this uncertainty by using the normalisation obtained by the Soudan 2 experiment. The best fit point for Soudan 2 resulted in a normalisation $5 \%$ higher than the Battistoni flux model with an estimated error of $\pm 10 \%$. Figure 7.21 shows the expected sensitivity for a perfectly known normalisation, a normalisation known to $10 \%$ and $20 \%$ and a shape only fit where the normalisation 
is allowed to float by removing the term containing $\sigma_{\alpha}$ from Equation 7.4. As can be seen in Figure 7.21 only marginal improvements in sensitivity are expected from a better understanding of the overall normalisation.

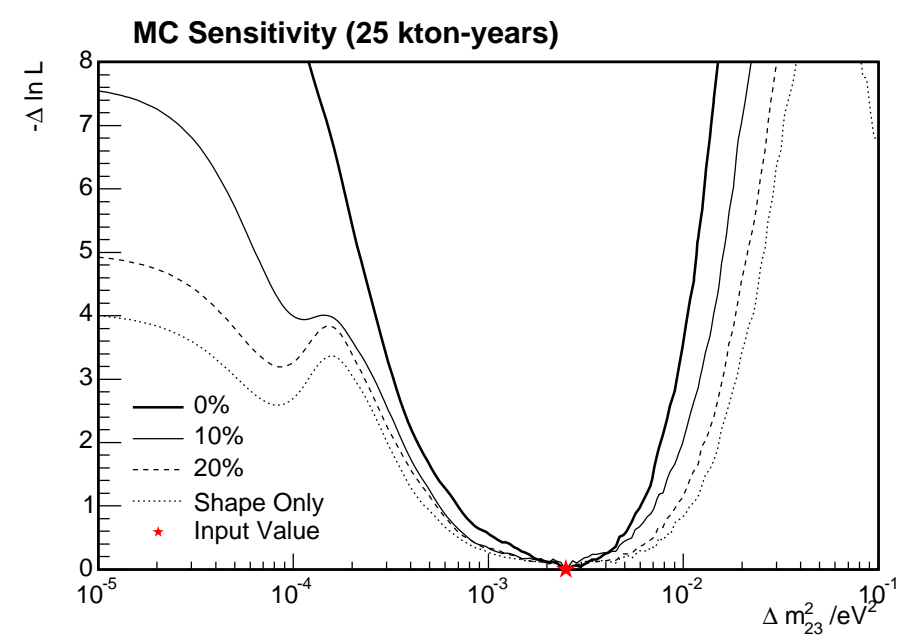

Figure 7.21: The effect on the sensitivity of the neutrino event normalisation uncertainty. The likelihood curves were obtained using the zenith angle/energy fitting method.

\subsubsection{Hadronic Models}

Studies at the MINOS Calibration Detector show that GCALOR gives results that best describe the hadronic response of the MINOS detectors to single pions and protons. However, other models were tested, in particular SLAC-GHEISHA and FLUKA. Of the two alternative models, SLAC-GHEISHA was found to give better agreement with data 92 .

The effect of the hadronic model on event selection and parameter estimation is expected to be small as most of the information about the event comes from the muon track. Two Monte Carlo samples were generated using SLAC-GHEISHA and FLUKA, each corresponding to a 344 kton-year exposure. Events were selected using the same set of selection cuts described in the previous chapter. The zenith angle and energy distributions were compared to those obtained using GCALOR. Figure 7.22 shows the distribution of reconstructed energies of selected events for the three models. The SLAC-GHEISHA and FLUKA models both reproduce the distribution obtained using GCALOR to within statistical errors. The agreement in the en- 
ergy distribution from GCALOR and SLAC-GHEISHA is $\operatorname{good}\left(\chi^{2} / n d f=25.1 / 20\right)$. Good agreement is also seen between GCALOR and FLUKA $\left(\chi^{2} / n d f=20.1 / 20\right)$. Figure 7.23 shows the zenith angle distributions for all models in three energy ranges for events with good timing and the Low Resolution sample. The SLAC-GHEISHA model reproduced the GCALOR distributions with a value $\chi^{2} / n d f$ of $50.6 / 46$. By comparing the FLUKA and GCALOR distributions a $\chi^{2} / n d f=52.9 / 46$ was calculated. It should be noted that the distributions have not been renomalised, so the quoted level of agreement includes systematic differences in selection efficiencies between the models. The two alternative Monte Carlo samples were generated using different events to the GCALOR sample, therefore agreement between the models is consistent with statistical fluctuations rather than a systematic effect. It is concluded that the effect of hadronic model on the measurement of oscillation parameters in this analysis is small.

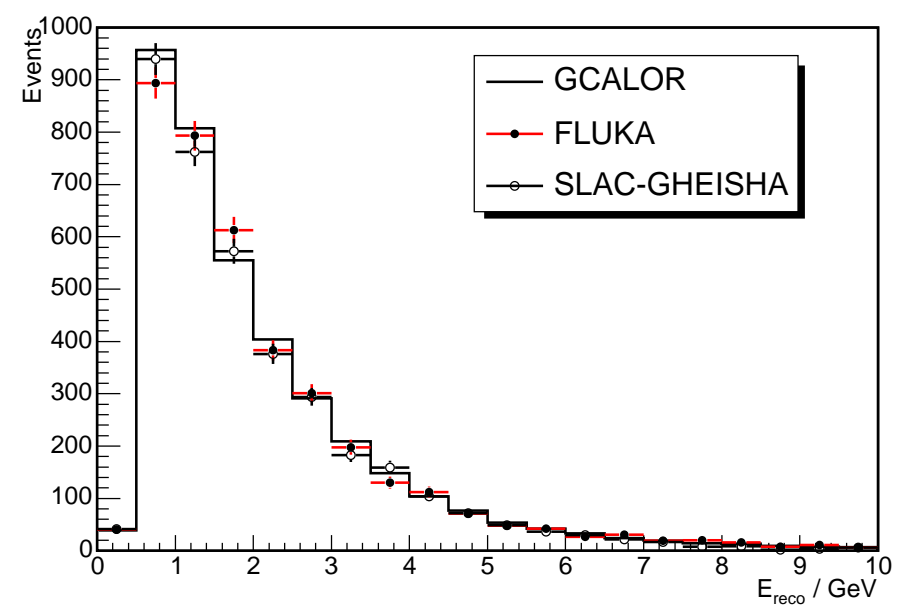

Figure 7.22: The reconstructed energies of selected Monte Carlo events using different hadronic models. Statistical errors only are shown. The statistical errors on the GCALOR sample are small. 

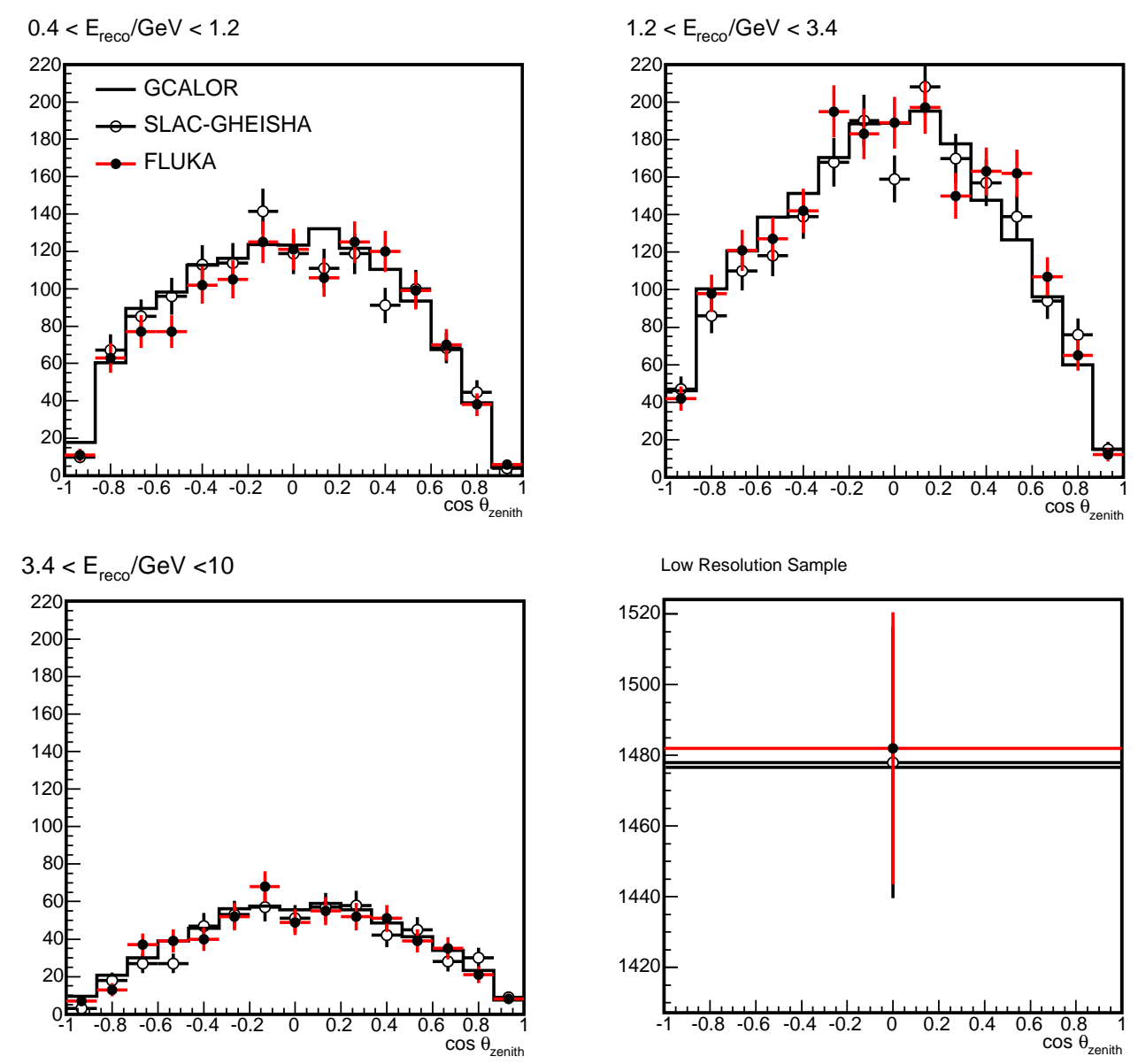

Figure 7.23: The reconstructed zenith angle distributions for Monte Carlo events generated with three different hadronic interaction models. Statistical errors only are shown. 


\subsubsection{Event Timing}

It was shown in Section 5.2 that there was a significant difference between data and Monte Carlo event timing. The same technique discussed in Section 7.1.3 was used to degrade the Monte Carlo timing to match the data. Figure 7.24 shows the expected sensitivity for 25 kton-years of data before and after the timing smearing has been applied to the simulated data set. The effect of the smearing is to introduce a small bias towards a null oscillation result as it randomises the direction of a some of the tracks, washing out the oscillation signature. However, the effect is small as most of the events with poor timing are in the Low Resolution sample.

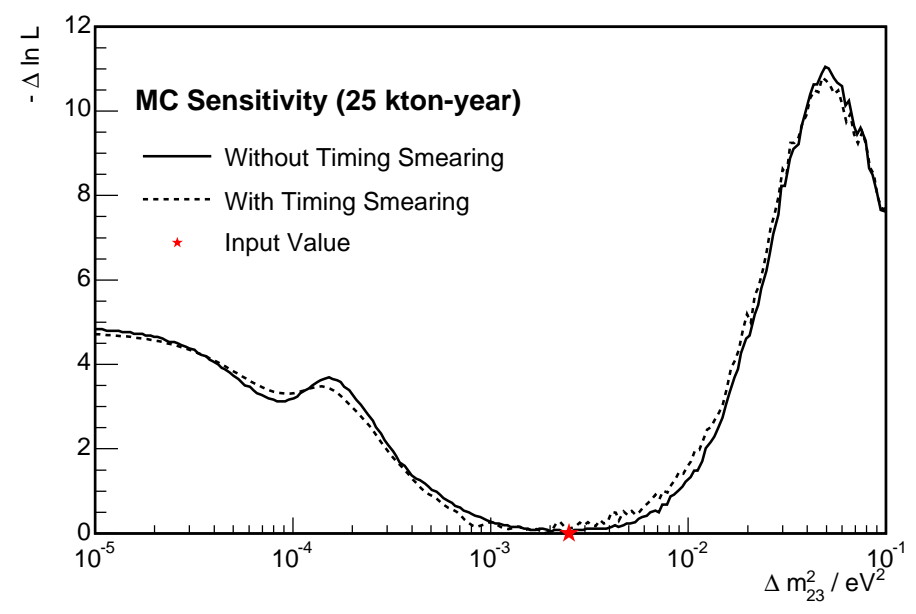

Figure 7.24: The effect of smearing the event timing simulation on the expected sensitivity.

\subsubsection{Neutrino Cross Sections}

The selection cuts are biased towards events with low $Q^{2}$. As a result, $60 \%$ of the selected events are quasi-elastic. However, there is little experimental data in this region to tune the neutrino interaction models. A conservative estimate of the systematic uncertainty in the contribution of the quasi-elastic process to the total cross section is $20 \%$ [78]. Figure 7.25 shows the effect of varying the contribution of quasi-elastic events in the simulated data set by $20 \%$ in both directions. The 
effect is to introduce a bias in the best fit point due to the change in the energy spectrum of selected events. The size of the effect is significant and the uncertainty in the quasi-elastic contribution is included as a systematic uncertainty in the final parameter estimation.

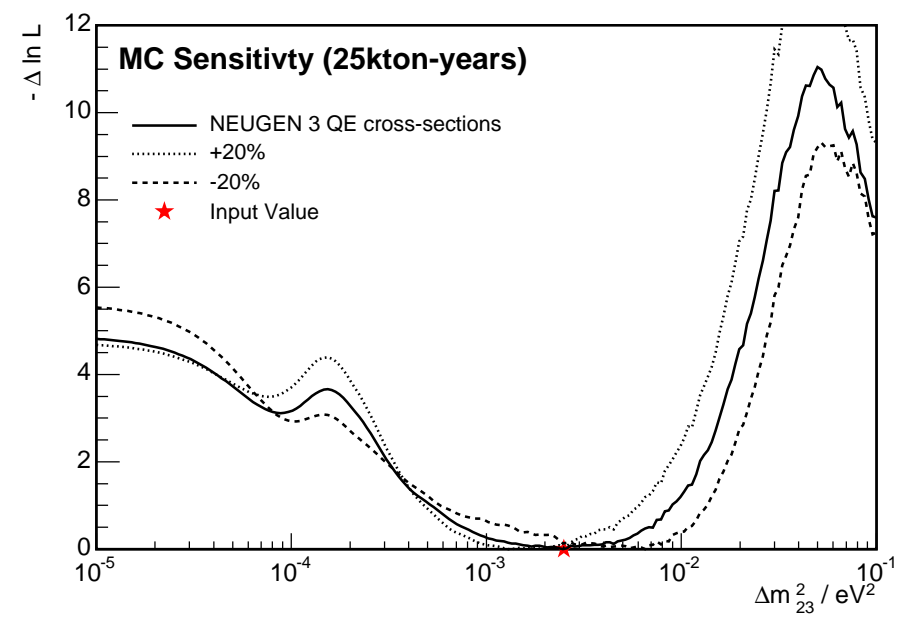

Figure 7.25: The effect of the systematic uncertainty on the contribution of the quasi-elastic process to the total cross section on the expected sensitivity.

\subsection{Expected 25 kton-year Sensitivity}

The zenith angle/energy fitting method (discussed in Section 7.3) was used to obtain the expected sensitivity for an exposure of 25 kton-years. The correction to the timing resolution was made for both Monte Carlo templates and simulated data. The expected distributions are shown in Figure 7.19. To include the effect of the uncertainty on the quasi-elastic cross section, the relative contribution from quasi-elastic events was introduced into the fit as an extra nuisance parameter. The likelihood in Equation 7.4 is modified to,

$$
\begin{gathered}
-\ln L=\sum_{\text {bins }(i)}\left(\alpha\left(N_{i}^{\nu}+\gamma N_{i}^{Q E}\right)+\beta N_{i}^{\mu}+\ln \left(N_{i}^{\text {data }}\right)-N_{i}^{\text {obs }} \ln \left(\alpha\left(N_{i}^{\nu}+\gamma N_{i}^{Q E}\right)+\beta N_{i}^{\mu}\right)\right) \\
+\frac{(1-\alpha)^{2}}{\sigma_{\alpha}^{2}}+\frac{(1-\beta)^{2}}{\sigma_{\beta}^{2}}+\frac{(1-\gamma)^{2}}{\sigma_{\gamma}^{2}}
\end{gathered}
$$


where $N_{i}^{Q E}$ is the contribution to the $i^{t h}$ bin from quasi-elastic signal events and $N_{i}^{\nu}$ is the contribution to the bin from all other atmospheric neutrino events. $N_{i}^{\mu}$ is the contribution from the cosmic muon background. The uncertainty in the neutrino and cosmic muon flux is represented by $\sigma_{\alpha}$ and $\sigma_{\beta}$. The uncertainty in the quasi-elastic contribution is given by $\sigma_{\gamma}$ and is assigned a value of $20 \%$.

Figure 7.26 shows the expected sensitivity for a 25 kton-year exposure. The $90 \%$ and $95 \%$ confidence limits are calculated assuming a 2-dimensional Gaussian approximation and taking the limits at $-\ln L=-(\ln L)_{\min }+2.31(90 \%)$ or $+3.00(95 \%)$ [6], where $-\ln L_{\min }$ is the value of $-\ln L$ at the best fit point.

The MiniBOONE, K2K and MINOS (Near Detector) experiments will significantly increase the understanding of neutrino cross sections in the few $\mathrm{GeV}$ region. In addition, improved Far Detector timing calibrations will give significantly improved timing resolutions. Figure 7.26 shows the limits obtained when the uncertainty on the quasi-elastic contribution and the smearing of the timing resolution are removed. the effect on the expected sensitivity is relatively small.

\subsection{Fit to Data}

Figure 7.27 shows the zenith angle distributions of the selected data events compared to Monte Carlo for both the unoscillated and oscillated $\left(\sin ^{2} 2 \theta=1, \Delta m_{23}^{2}=\right.$ $0.0025 \mathrm{eV}^{2}$ ) hypotheses. The confidence bands for $\sin ^{2} 2 \theta$ and $\Delta m_{23}^{2}$ were obtained directly using the Feldman and Cousins construction (FC) [93]. This method has become widely adopted by neutrino oscillations experiments (for example [29] and [13]) as it has a number of attractive features; no assumption about the Gaussian nature of errors is made, it correctly deals with physical boundaries and it allows the natural inclusion of systematic uncertainties. It is based on the likelihood ratio ordering principle, a full description of which is given in [93]. The FC approach has the disadvantage that it is computationally expensive. The CPU time scales as $N_{\text {exp }} \times N_{p} \times N_{p}$, where $N_{\text {exp }}$ is the number of simulated experiments and $N_{p}$ is the number of points in parameter space. For this reason it was not used to calculate the sensitivity plots.

To determine the oscillation parameters, the data distributions shown in Figure 7.27 are compared to the expected templates for each point on the $\Delta m_{23}^{2}, \sin ^{2} 2 \theta$ 


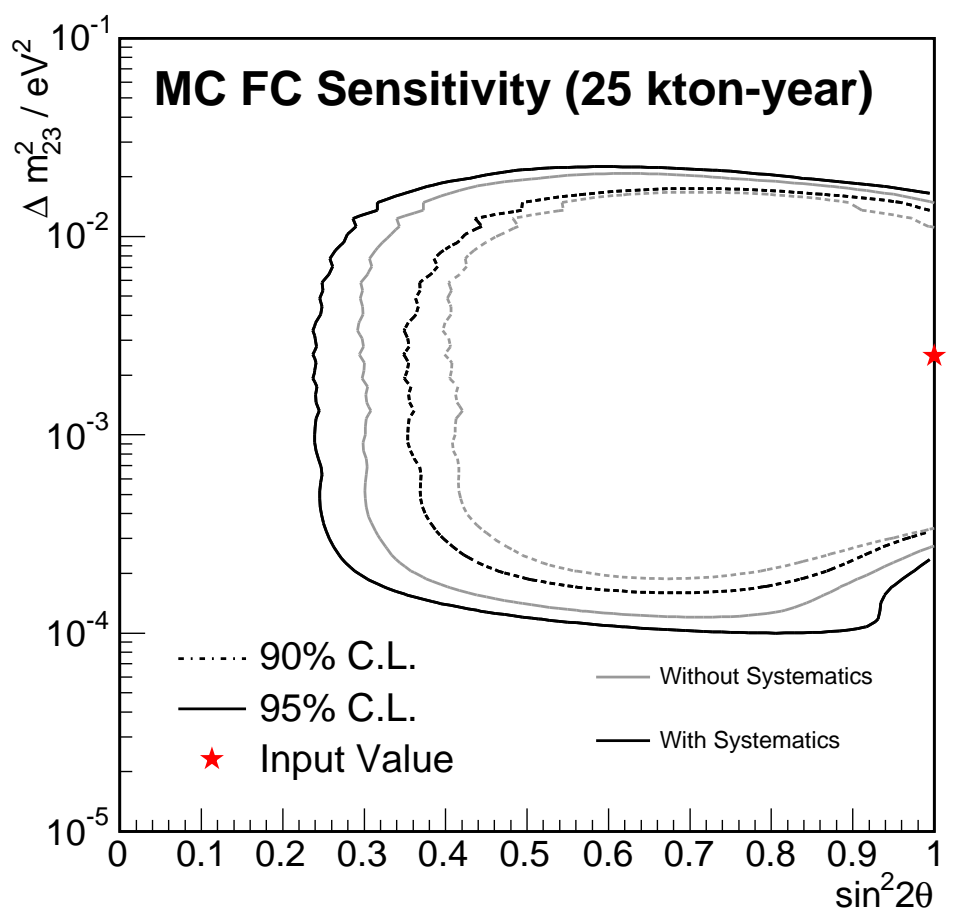

Figure 7.26: The average expected confidence limits obtained by the fully contained analysis for 25 kton-years of data. The input value of $\theta=\pi / 4$ and $\Delta m_{23}^{2}=0.0025 \mathrm{eV}^{2}$ is shown by the star. The mean $90 \%$ and $95 \%$ confidence limits are shown by the dashed and solid line, respectively. The limits obtained when all systematics apart from signal normalisation are removed are also shown.

plane. The likelihood at each point on the plane is calculated as

$$
\mathcal{L}=-\ln \mathrm{L}=\sum_{i=1}^{\text {bins }} N_{i}^{M C}+\ln N_{i}^{\text {data }} !-N_{i}^{\text {data }} \ln N_{i}^{M C}
$$

where $N_{i}^{M C}$ is the total number of events (signal and backgrounds) in the $i^{\text {th }}$ bin from Monte Carlo and $N_{i}^{\text {data }}$ is the number observed. The best fit point occurs at $\sin ^{2} 2 \theta=1.0$ and $\Delta m_{23}^{2}=0.0001 \mathrm{eV}^{2}$. For each point $(j, k)$ in the $\left(\sin ^{2} 2 \theta \Delta m_{23}^{2}\right)$ space the quantity $(\Delta \mathcal{L})_{j k}=\mathcal{L}_{j k}-\mathcal{L}_{\text {best }}$ is constructed, where $\mathcal{L}_{\text {best }}$ is the value of $\mathcal{L}$ at the best fit point. The resultant surface is shown in Figure 7.28,

The confidence limits are then calculated using the FC approach. At each grid point $(j, k)$, a Monte Carlo experiment is generated with the appropriate statistical 
and systematic variations included. The systematic errors included are the normalisation of the atmospheric neutrino flux (20\%), the normalisation of the cosmic ray muon flux (15\%) and the quasi-elastic contribution $(20 \%)$. The difference between $\mathcal{L}$ at that grid point and the minimum value of $\mathcal{L}$ anywhere in the allowed region, which is not necessarily at $(j, k)$, is calculated, $\Delta \mathcal{L}$. This procedure is repeated 1000 times to obtain a distribution of $\Delta \mathcal{L}$. The assumption is made that if the true parameters had been those at the grid point $(j, k)$, then the real experiment would have produced a likelihood drawn from the distribution obtained. Therefore, the $68 \%$ confidence limit (for example) for this point will contain $68 \%$ of the generated experiments. The $68 \%$ limit can therefore be obtained by integrating the distribution of $\Delta \mathcal{L}$ until 680 of the generated experiments have been included. If $\Delta \mathcal{L}$ for the data at $(j, k)$ is less than this limit, then $(j, k)$ lies within the $68 \%$ confidence limit. The confidence bands obtained are shown in Figure 7.29 .

It is clear from Figure 7.29 that there is no significant evidence of neutrino oscillations in the data analysed in this thesis. The consistency with Super-Kamiokande's best fit point $\left(\Delta m_{23}^{2}=0.0024, \sin ^{2} 2 \theta=1.0\right)$ has been tested by generating a large number of mock data samples (including appropriate statistical and systematic errors) with equivalent exposure to the data assuming Super-Kamiokande's oscillation parameters. In $9 \%$ of the generated samples the likelihood obtained when compared to Monte Carlo at $\left(\Delta m_{23}^{2}=0.0024 \mathrm{eV}^{2}, \sin ^{2} 2 \theta=1.0\right)$ exceeded that obtained when comparing the data. Therefore, the data is consistent with the hypotheses of no oscillations and oscillations with parameters equal to the best fit value obtained by Super-Kamiokande. 

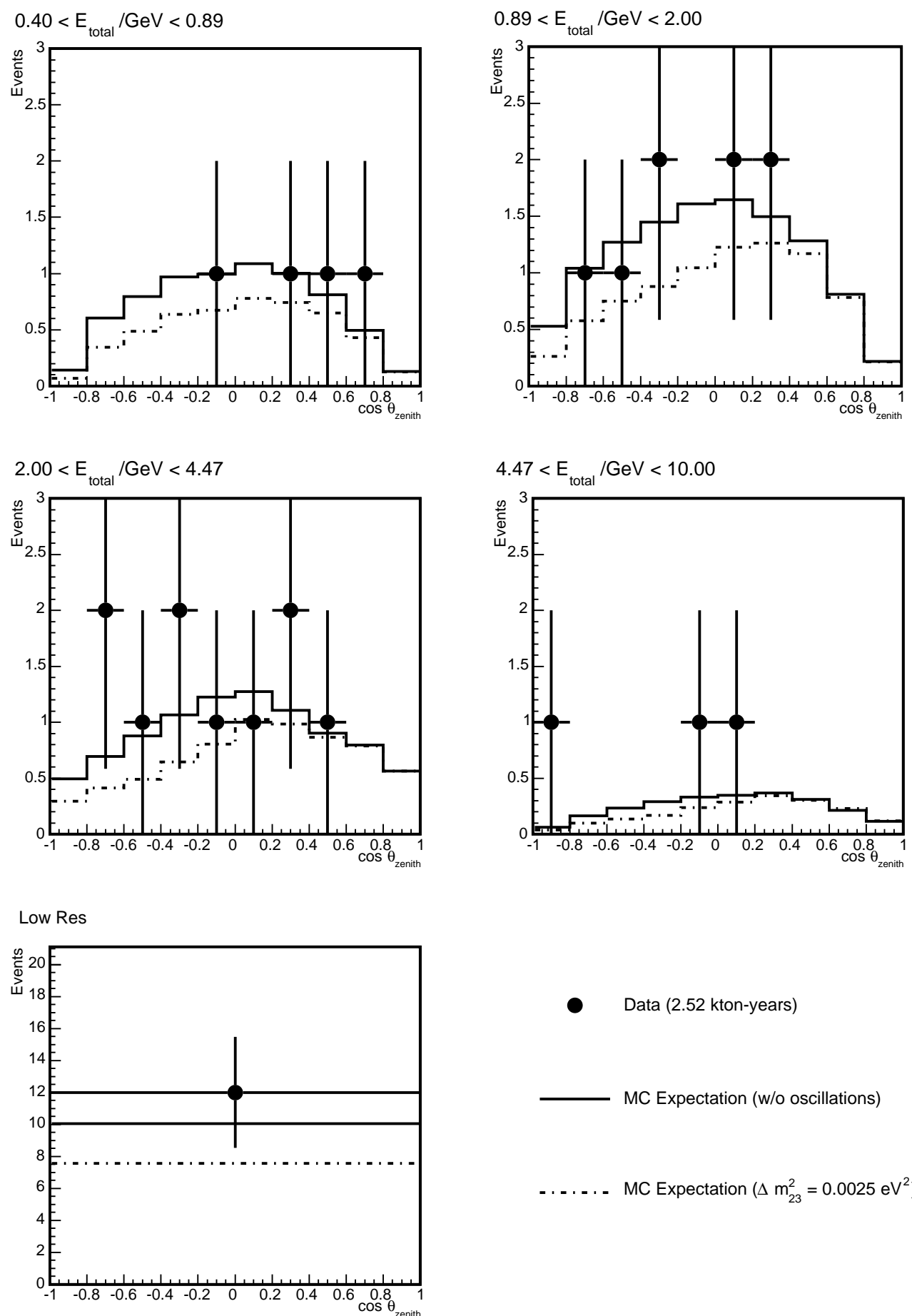

Data (2.52 kton-years)

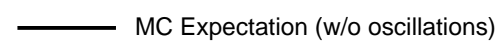

- . . . . MC Expectation $\left(\Delta \mathrm{m}_{23}^{2}=0.0025 \mathrm{eV}^{2}\right)$

Figure 7.27: The expected and observed zenith angle distributions of the Good Timing sample for four energy ranges and the Low Resolution sample. 

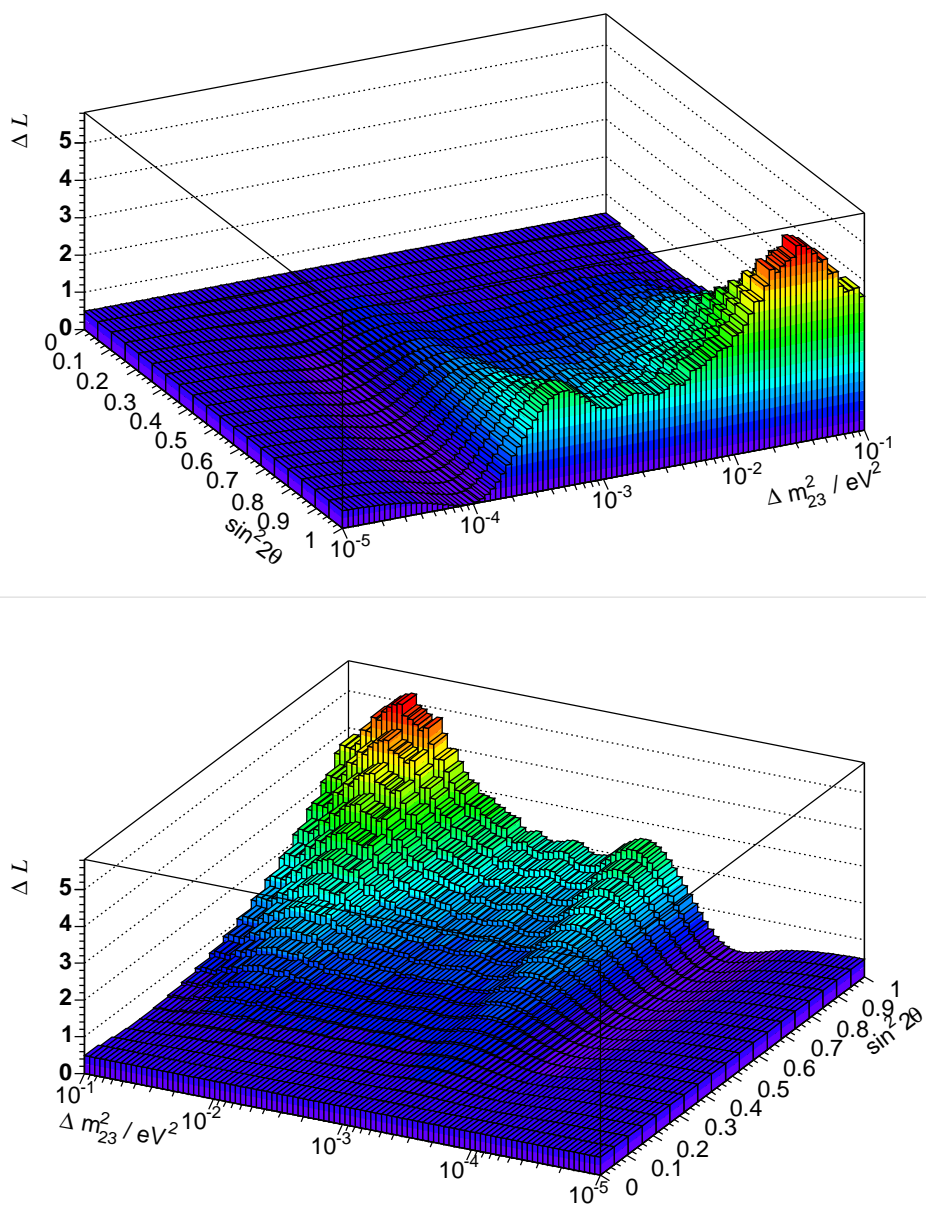

Figure 7.28: Two views of the $\Delta \mathcal{L}$ surface obtained for the selected events. There are 20 bins in $\sin ^{2} 2 \theta$ and 100 in $\Delta m_{23}^{2}$. The value of $\Delta \mathcal{L}$ in the bin $(i, j)$ is obtained from $\mathcal{L}_{i j}-\mathcal{L}_{\text {best }}$, where $\mathcal{L}_{\text {best }}$ is the minimum value of $-\ln \mathrm{L}$. 


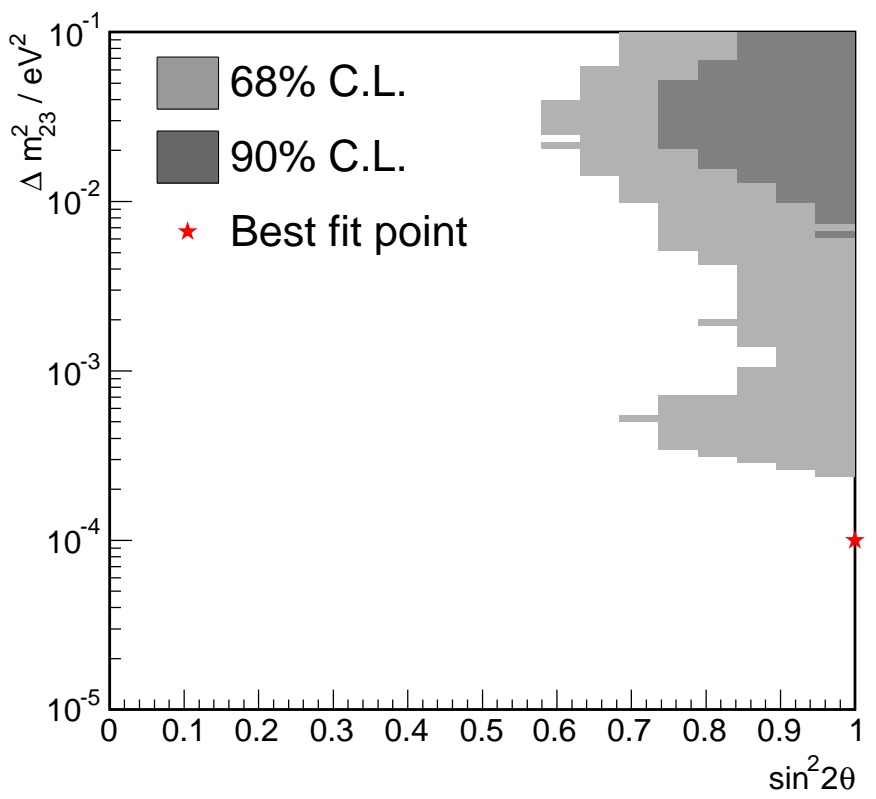

Figure 7.29: The $68 \%$ and $90 \%$ excluded regions for selected data events in 2.52 ktonyears of data defined using FC method. Points outside the shaded regions are allowed. The best fit point at $\sin ^{2} 2 \theta=1.0, \Delta m_{23}^{2}=0.0001 \mathrm{eV}^{2}$ is shown by the marker. 


\subsection{Charge Separated Oscillation Measurement}

As discussed in Section 7.1.3 the detector's magnetic field can be used to separate the charge of the selected events with a high purity and hence limits on the oscillation parameters for $\nu_{\mu}\left(\sin ^{2} 2 \theta, \Delta m_{23}^{2}\right)$ and $\bar{\nu}_{\mu}\left(\sin ^{2} 2 \bar{\theta}, \Delta \bar{m}_{23}^{2}\right)$ can be obtained.

The zenith angle distributions of the selected events when separated into $\nu_{\mu}$ and $\bar{\nu}_{\mu}$ are shown in Figure 7.30. The selected events were subjected to Feldman and Cousins likelihood analysis to measure the oscillation parameters, $\Delta m_{23}^{2}$ and $\Delta \bar{m}_{23}^{2}$. However, due to the low statistics no significant limits could be placed on the value of $\Delta \bar{m}_{23}^{2}$ at this time.

To calculate the expected sensitivity, the selected events are divided up into four samples; a low resolution sample for events that fail the timing cut, an unknown charge sample for events that pass the timing cuts but fail the charge identification cut, and two samples where the charge can be identified. The samples with good timing are histogrammed in zenith angle and energy, as before. The expected and observed distributions for a 2.52 kton-year data set are shown in Figure 7.30. A set of 500 mock data sets including all backgrounds and appropriate statistical variations is created with input parameters $\sin ^{2} 2 \theta=\sin ^{2} 2 \bar{\theta}=1.0$ and $\Delta m_{23}^{2}=\Delta \bar{m}_{23}^{2}=$ $0.0025 \mathrm{eV}^{2}$. Each mock data set is then compared to Monte Carlo expectations for each point on a $100 \times 100$ grid of $\log _{10}\left(\Delta m_{23}^{2}\right), \log _{10}\left(\Delta \bar{m}_{23}^{2}\right)$ assuming maximal mixing,

and a likelihood calculated. The likelihood is calculated using Equation 7.5, where the sum over bins now includes the different charge samples and the uncertainties in the neutrino and anti-neutrino cross sections are allowed to vary independently. The mean likelihood surface is calculated and the expected confidence limits are derived using a 2-dimensional Gaussian approximation as before. Figure 7.31 shows the expected sensitivity for a 25 kton-year exposure. Also shown is the sensitivity obtained when the neutrino and anti-neutrino cross sections are correlated and not allowed to float independently.

Figure 7.32 shows the confidence limits obtained when the input value of $\Delta m_{23}^{2}$ is fixed at $0.0025 \mathrm{eV}^{2}$ but the input value of $\Delta \bar{m}^{2}$ is changed to $0.1 \mathrm{eV}^{2}$ and $10^{-5} \mathrm{eV}^{2}$. Figures 7.31 and 7.32 demonstrate that with five years of data and the current systematic uncertainties the MINOS fully contained events will be able to resolve differences between $\Delta m_{23}^{2}$ and $\Delta \bar{m}_{23}^{2}$ at the level of two orders of magnitude. 

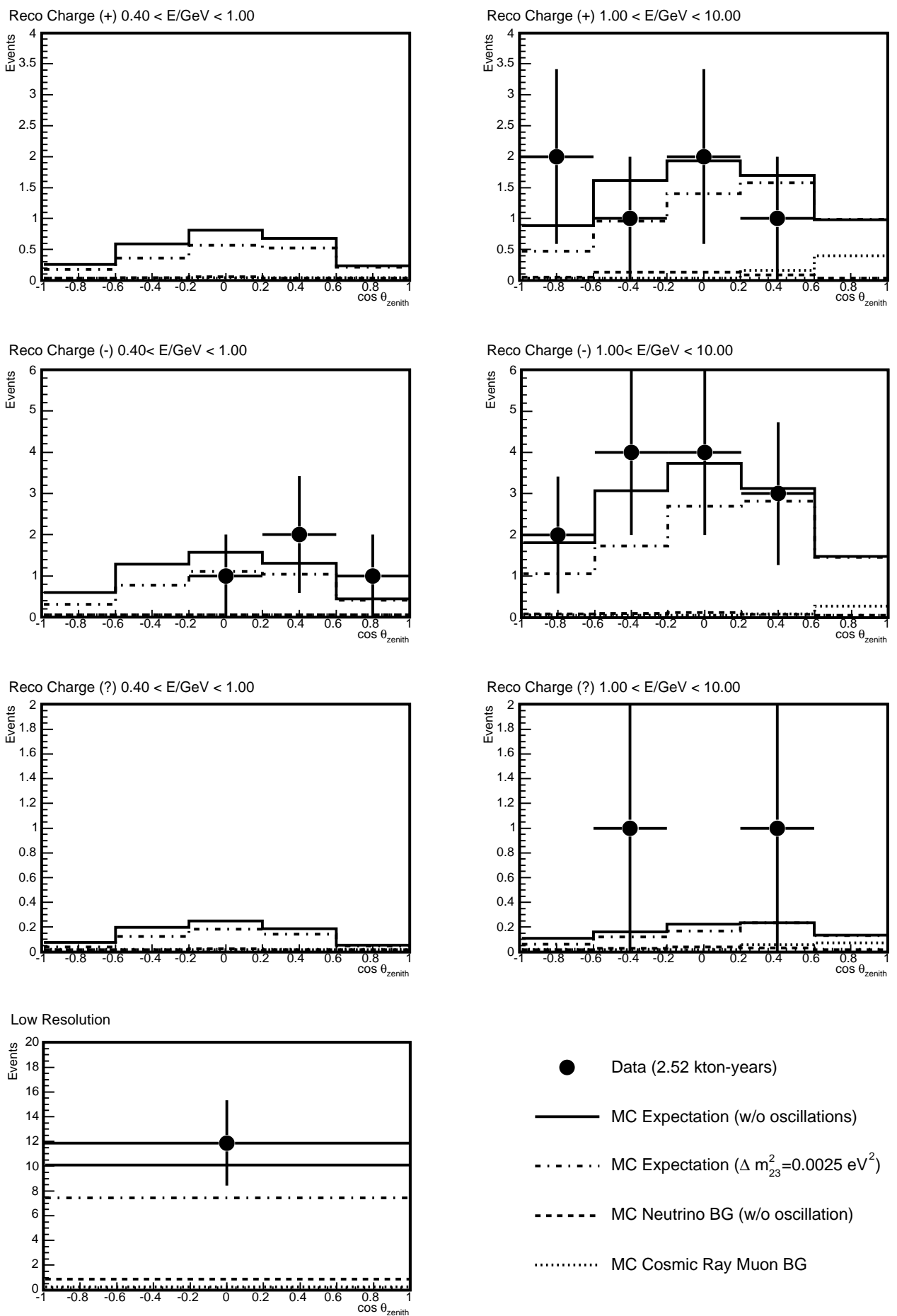

Figure 7.30: The expected and observed zenith angle distributions for 2.52 kton-years exposure. The expected distributions from Monte Carlo in the absence of oscillations is shown by the solid line. All histograms have been corrected for inaccuracies in the timing simulation. 


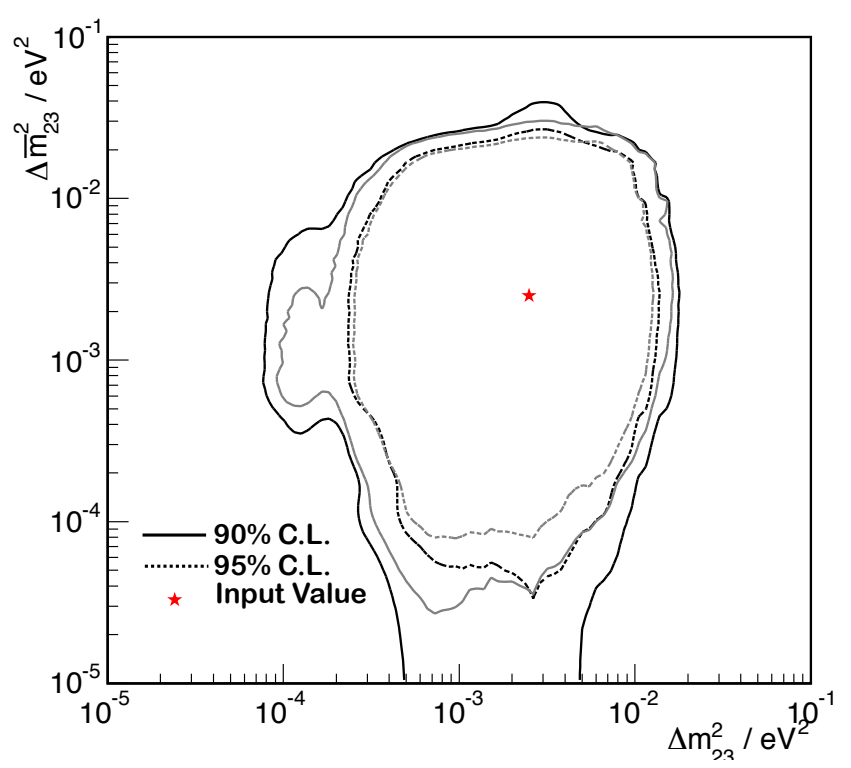

Figure 7.31: The average expected sensitivity to $\Delta m^{2}$ and $\Delta \bar{m}^{2}$ for 25 kton-years exposure of data. The expected sensitivity obtained when the normalisation of neutrinos and anti-neutrinos are not allowed to float independently is shown by the grey lines.
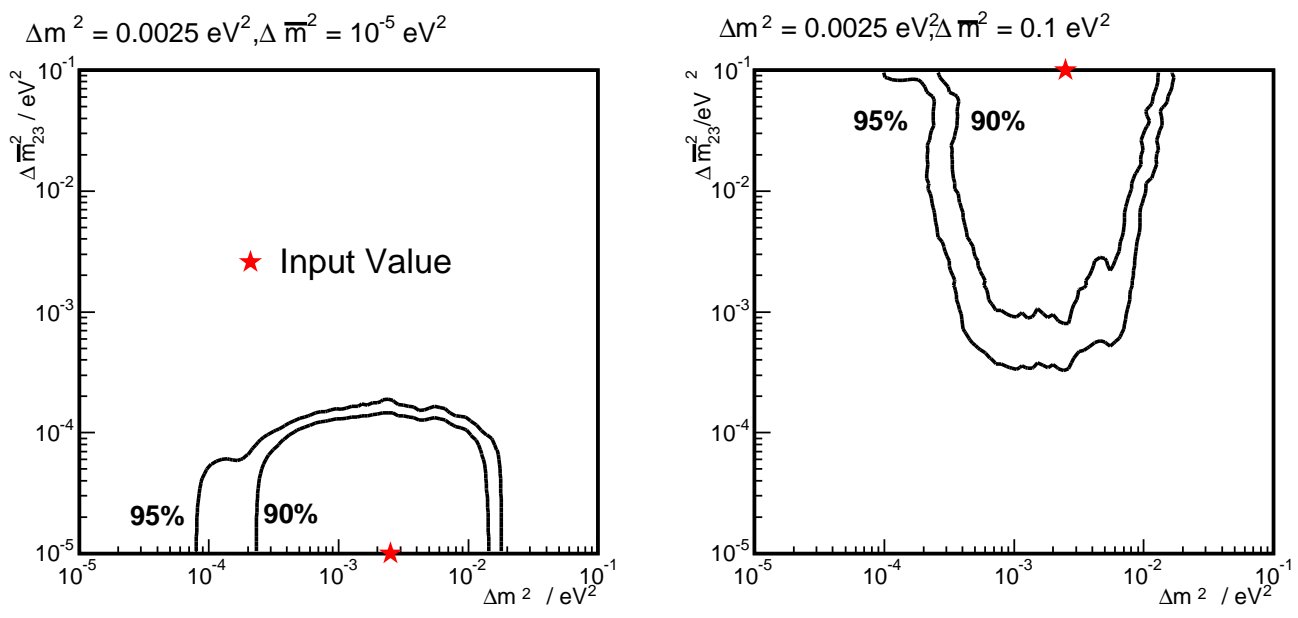

Figure 7.32: The average expected confidence limits from Monte Carlo samples equivailent to 25 kton-years exposure for two cases $\left(\Delta m_{23}^{2}, \Delta \bar{m}_{23}^{2}\right)=\left(0.0025 \mathrm{eV}^{2}, 10^{-5} \mathrm{eV}^{2}\right)$ and $\left(0.0025 \mathrm{eV}^{2}, 0.1 \mathrm{eV}^{2}\right)$. 


\subsection{Summary}

This chapter detailed the measurement of neutrino properties such as energy and direction. The direction of muon propagation along the reconstructed track was determined using the timing of hits along the track's length. Tracks with ambiguous timing information are identified and placed in a special low resolution sample. It was shown that the zenith angle resolution was dominated by the opening angle between the neutrino and the muon and not by event reconstruction. The momentum of the muon track was measured using the range of the track through the detector material. The energy of the hadronic shower was estimated by measuring the amount of energy deposited in the detector.

The MINOS far detector is the first deep underground neutrino detector with a magnetic field. Therefore, the charge of the muon produced can be identified on an event-by-event basis. The efficiency and purity of charge identification for contained events with unambiguous timing was investigated and found to be $92.4 \%$ and $95.1 \%$ respectively. When the charge selection cuts are applied to the data, $17 \nu_{\mu}$ and 6 $\bar{\nu}_{\mu}$ events were identified. There were 2 events with ambiguous charge and 12 events where the direction from timing could not be determined.

The selected events are analysed for a neutrino oscillation signature. However, due to the very low statistics of the sample, no significant statement about the oscillation parameters for either $\nu_{\mu}$ or $\bar{\nu}_{\mu}$ can be made. The average expected sensitivity after 5 years of running is presented and demonstrates that the MINOS contained atmospheric neutrino analysis will be able to observe differences between $\Delta m_{23}^{2}$ and $\Delta \bar{m}_{23}^{2}$ at the level of two orders of magnitude. 


\section{Chapter 8}

\section{Discussion}

The MINOS Far Detector was completed in August 2003 and since then has been collecting cosmic data. The data taken between August 2003 and April 2004 were analysed to separate atmospheric $\nu_{\mu}$ charged current interactions contained within the detector volume from the dominant background of cosmic ray muons. A total of 37 events were selected. Monte Carlo simulations predict 40.5 \pm 8.0 in the absence of oscillations and $29.7 \pm 5.9$ assuming $\sin ^{2} 2 \theta_{23}=1.0$ and $\Delta m_{23}^{2}=0.0025 \mathrm{eV}^{2}$. The veto shield was used to measure the background from cosmic ray muons, $1.5 \pm$ 0.002 (stat.) \pm 0.004 (sys.) which is in good agreement with Monte Carlo prediction of $1.8 \pm 0.2$ (stat.) \pm 0.3 (sys.).

The selected events were analysed using a maximum likelihood technique, for evidence of atmospheric neutrino oscillations. However, due to the very low statistics no significant statement about the oscillation parameters can be made at this time.

By measuring the curvature of the muon tracks in the magnetic field the charge of the muon can be determined with $95 \%$ purity and therefore identifying the neutrino event as a $\nu_{\mu}$ or $\bar{\nu}_{\mu}$ charged current interaction. A total of $17 \nu_{\mu}$ and $6 \bar{\nu}_{\mu}$ events were observed. The charge of 14 events could not be determined, mostly because the direction of the track from timing was ambiguous. This is the first observation of charge separated contained atmospheric neutrinos. The extremely limited statistics of the $\bar{\nu}_{\mu}$ sample means that no statement about the independence of the $\nu_{\mu}$ and $\bar{\nu}_{\mu}$ oscillation parameters can be made at this time.

The expected sensitivity for an exposure of 25 kton-years is presented for both charge separated and charge un-separated analyses. The sensitivity is calculated assuming current experimental resolutions and understanding of systematic errors. The studies show that the MINOS fully contained data set should be able to detect 
differences in $\Delta m_{23}^{2}$ and $\Delta \bar{m}_{23}^{2}$ of the order of one to two orders of magnitude. Therefore, the contained data set will be able to resolve the difference in $\Delta m_{23}^{2}$ and $\Delta \bar{m}_{23}^{2}$ required by the disagreement between the LSND and atmospheric neutrino results.

It should be noted that the presented sensitivity should not be interpreted as the final expected sensitivity of the MINOS atmospheric neutrino analysis. The analysis presented in this thesis only considers fully contained $\nu_{\mu}$ charged current interactions. However, there are additional event samples which are currently being examined which will contribute to the overall MINOS atmospheric neutrino sensitivity. The additional samples are muons from interactions in the rock below the detector, interactions within the detector and where the muon exits the detector (partially contained events) and electron neutrino interactions. Secondly, current efforts to improve the timing resolution will result in recovery of information from the Low Resolution sample. Therefore, the sensitivity presented in the previous chapter should not be directly compared to current allowed regions presented by the Super-Kamiokande, Soudan 2 and MACRO collaborations.

The MINOS atmospheric neutrino analysis will continue over the next four years. The start of beam data taking, scheduled for January 2005, is not expected to significantly reduce the amount of data available for atmospheric neutrino analyses.

Although the limited size of the data presented in this thesis means that no significant statement about oscillation parameters can be made, this thesis details the first direct observation of charge separated atmospheric neutrino interactions. It also details many of the techniques for performing atmospheric neutrino studies in the MINOS detector that have been developed. The analysis of atmospheric neutrino data has also led to a much improved understanding of the Far Detector before the NuMI beam is commissioned. 


\section{Appendix A}

\section{Selected Events}

The 37 data events selected are shown in chronological order below. For each event the run number and event number are shown in the upper left hand corner. The measured muon momentum and the energy of the hadronic shower (if observed) is also shown. The left hand panel shows the $U-Z$ view and the middle panel shows the $V-Z$ view. The right hand panel shows a $X-Y$ view obtained by combining the $U-Z$ and $V-Z$ views. In each view the estimated vertex position is shown by the red star.
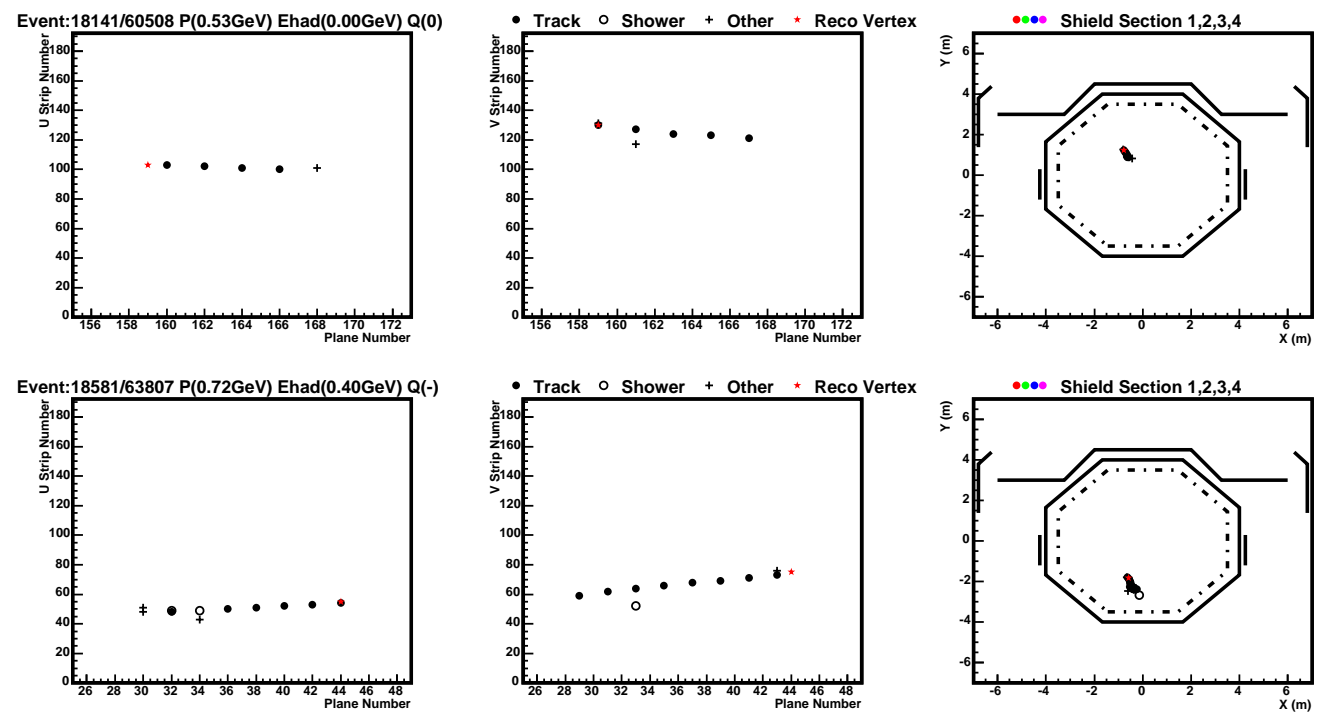

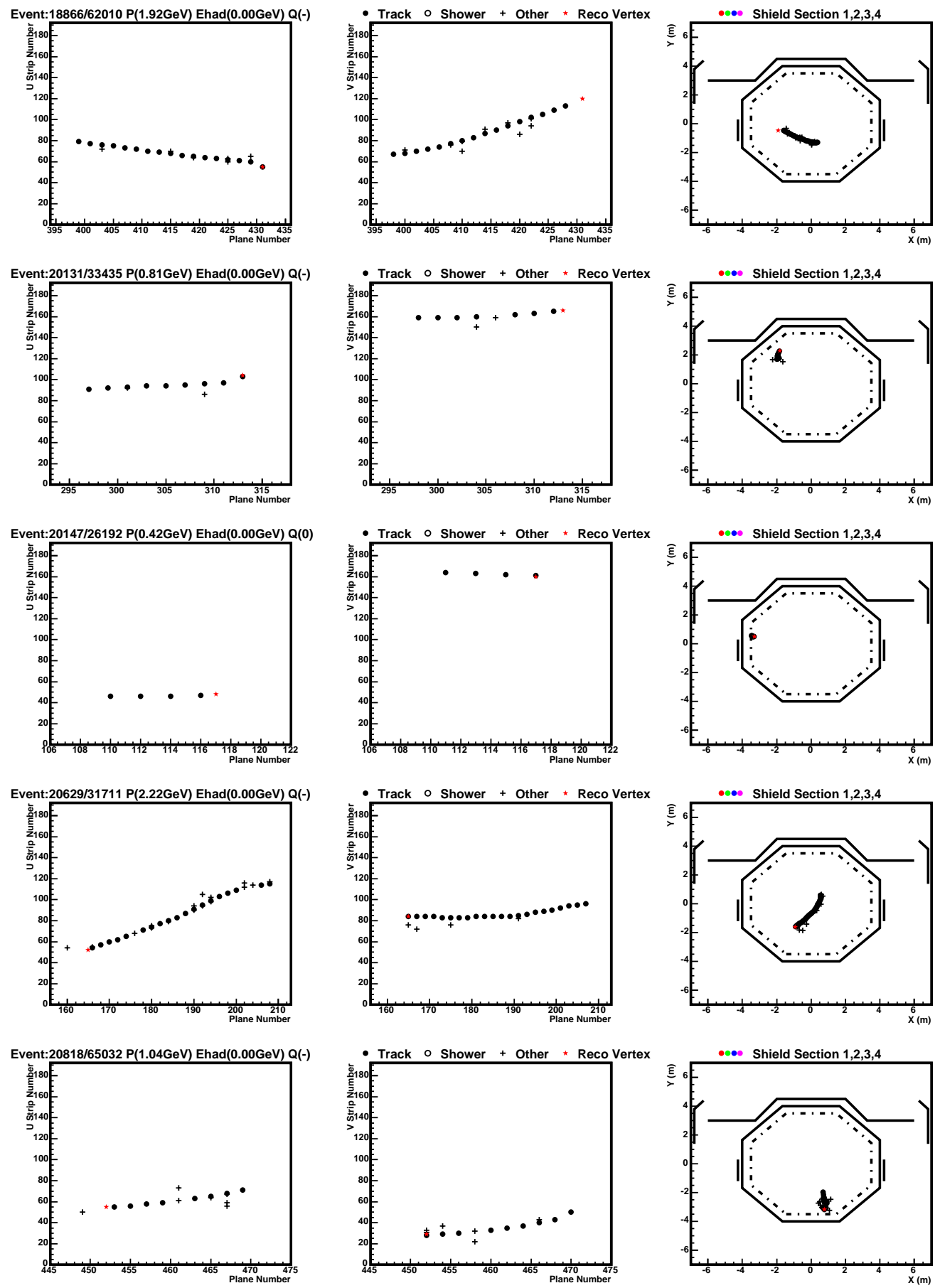

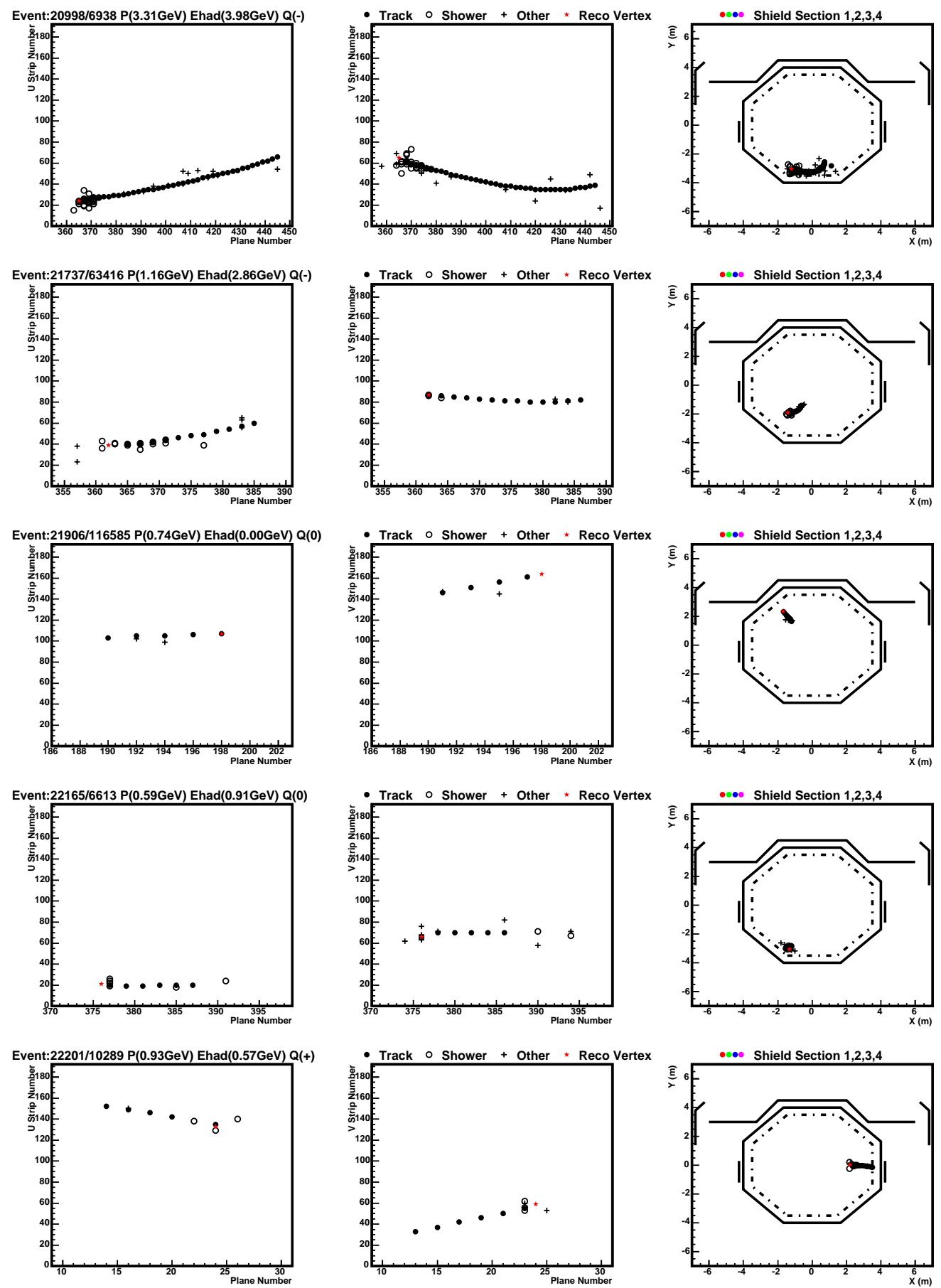

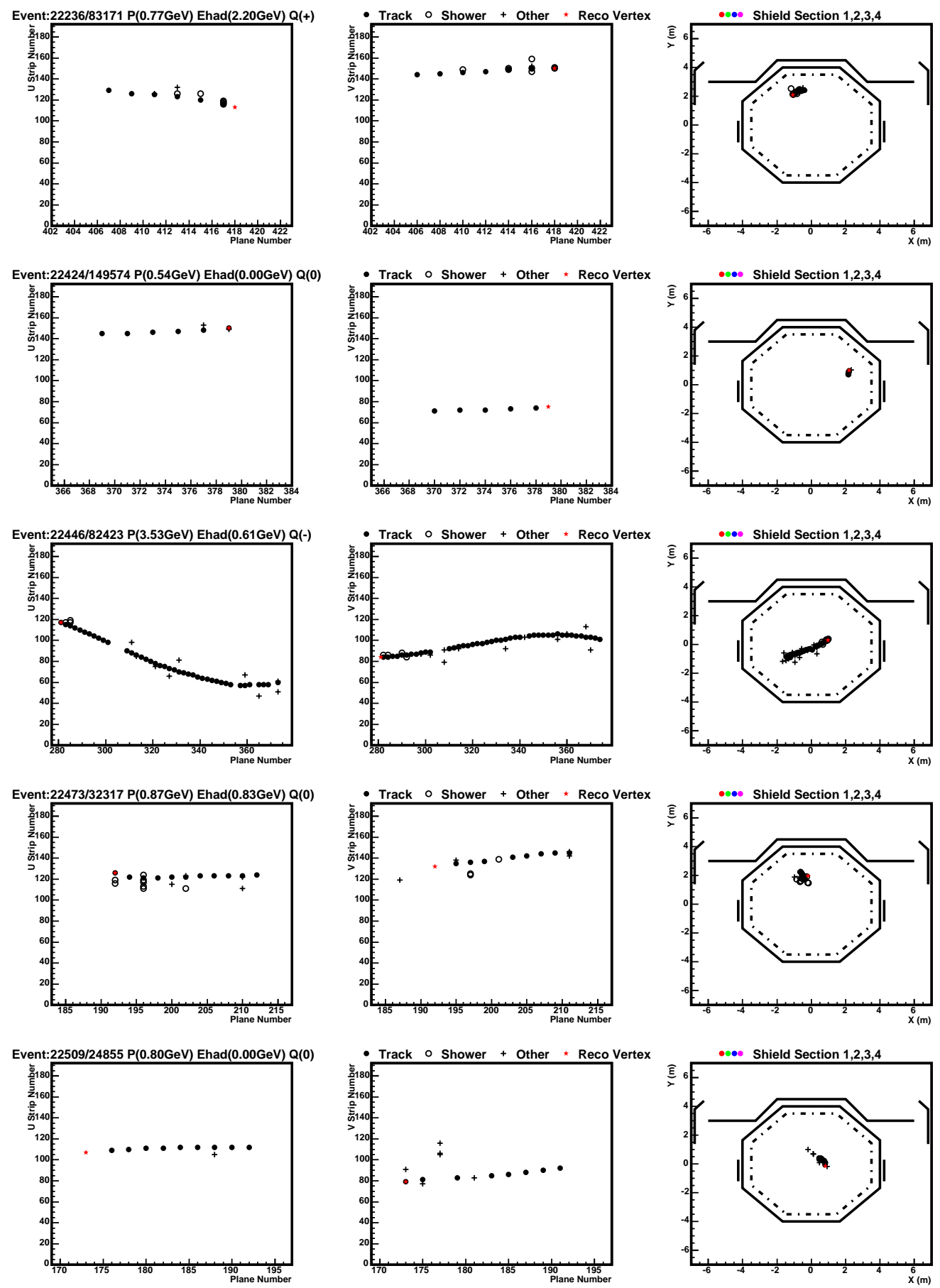

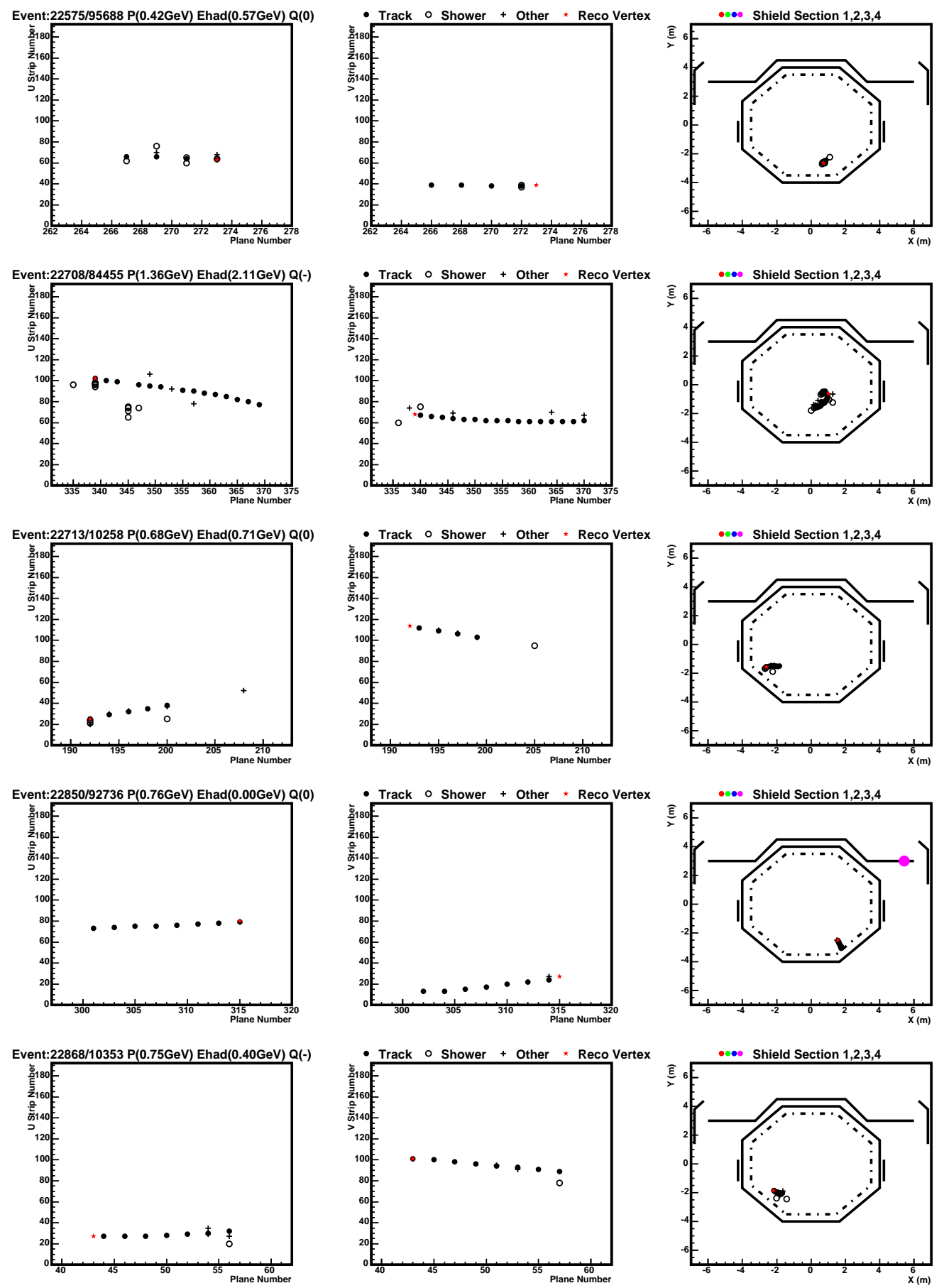

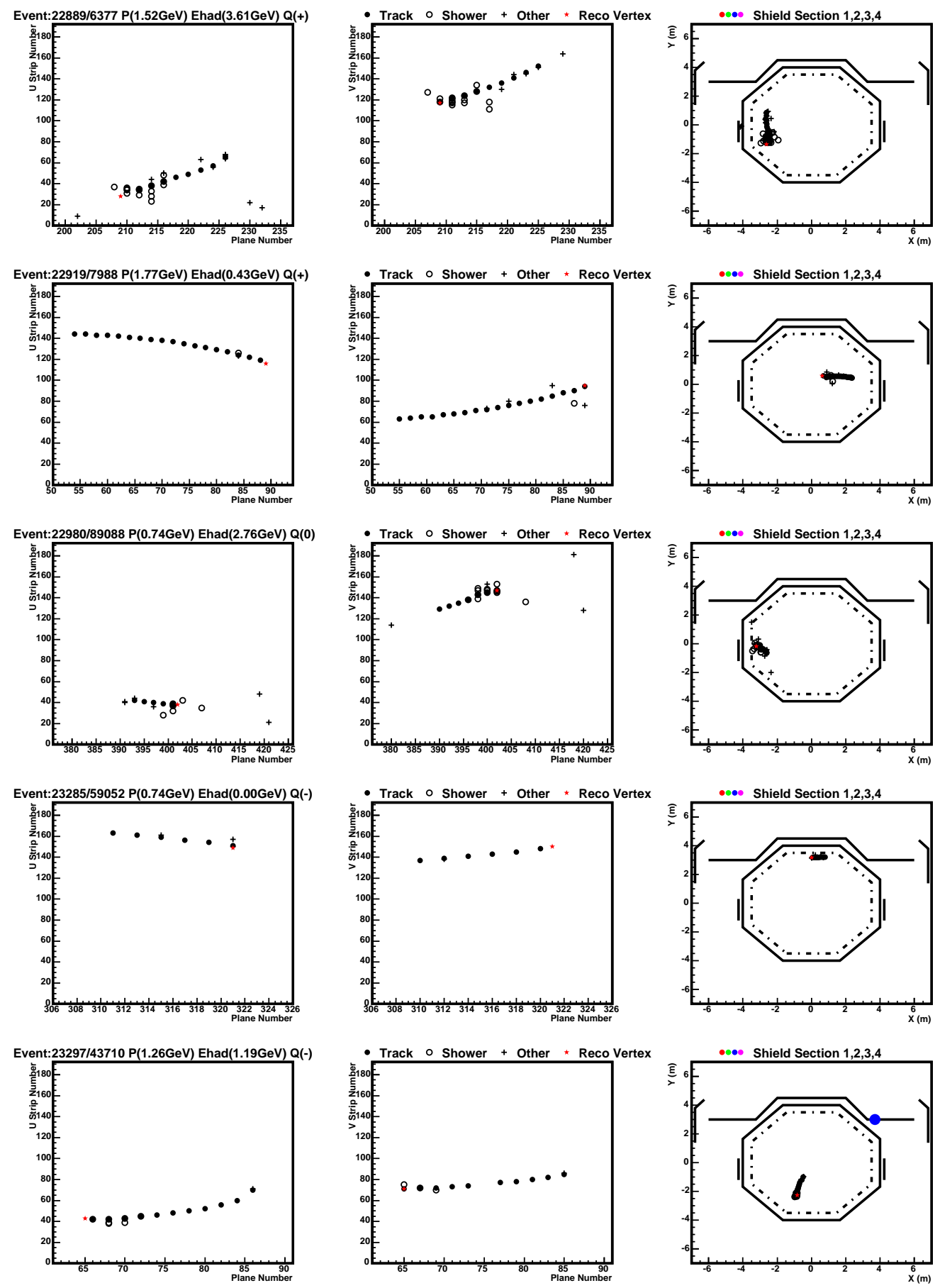

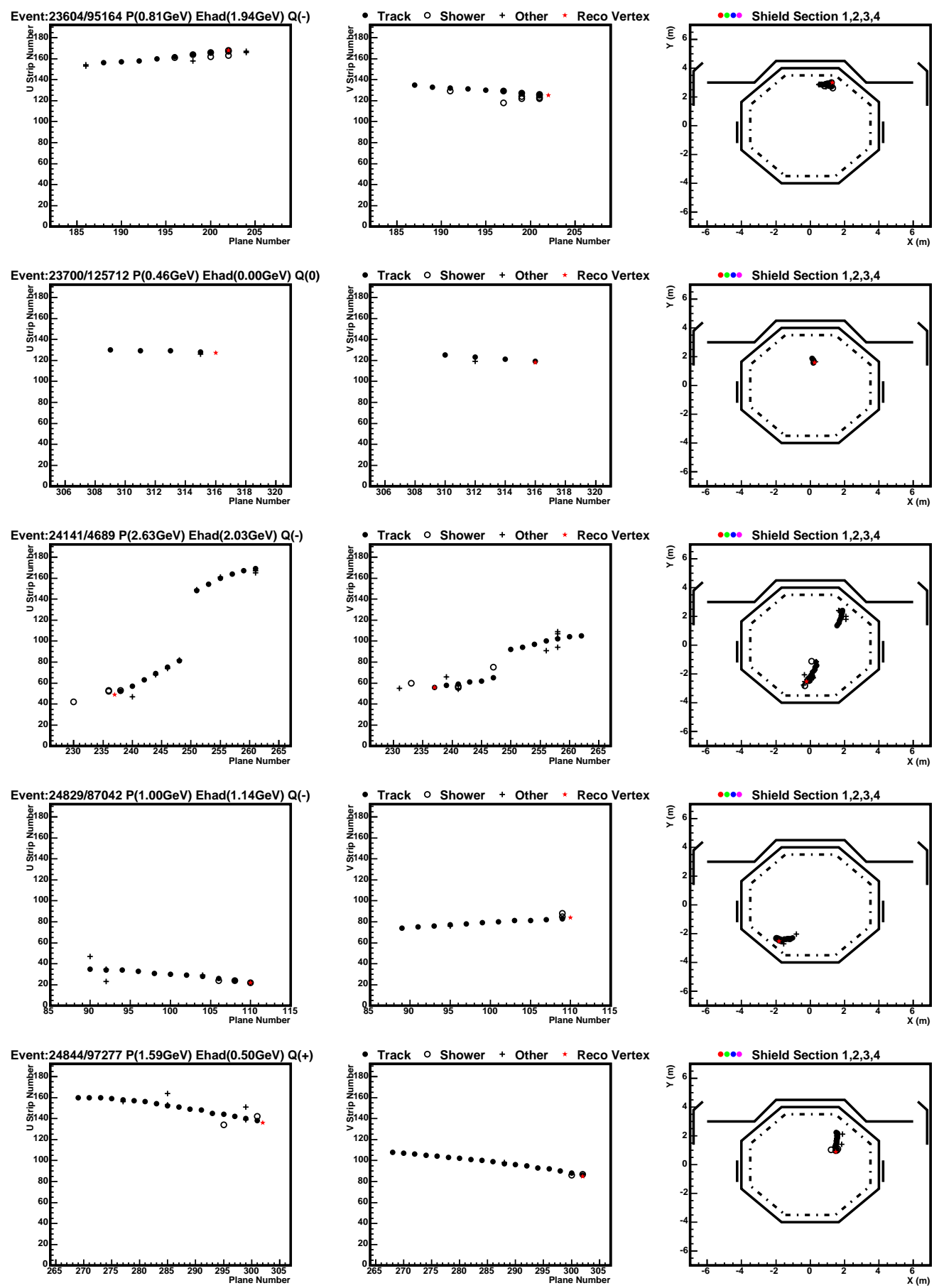

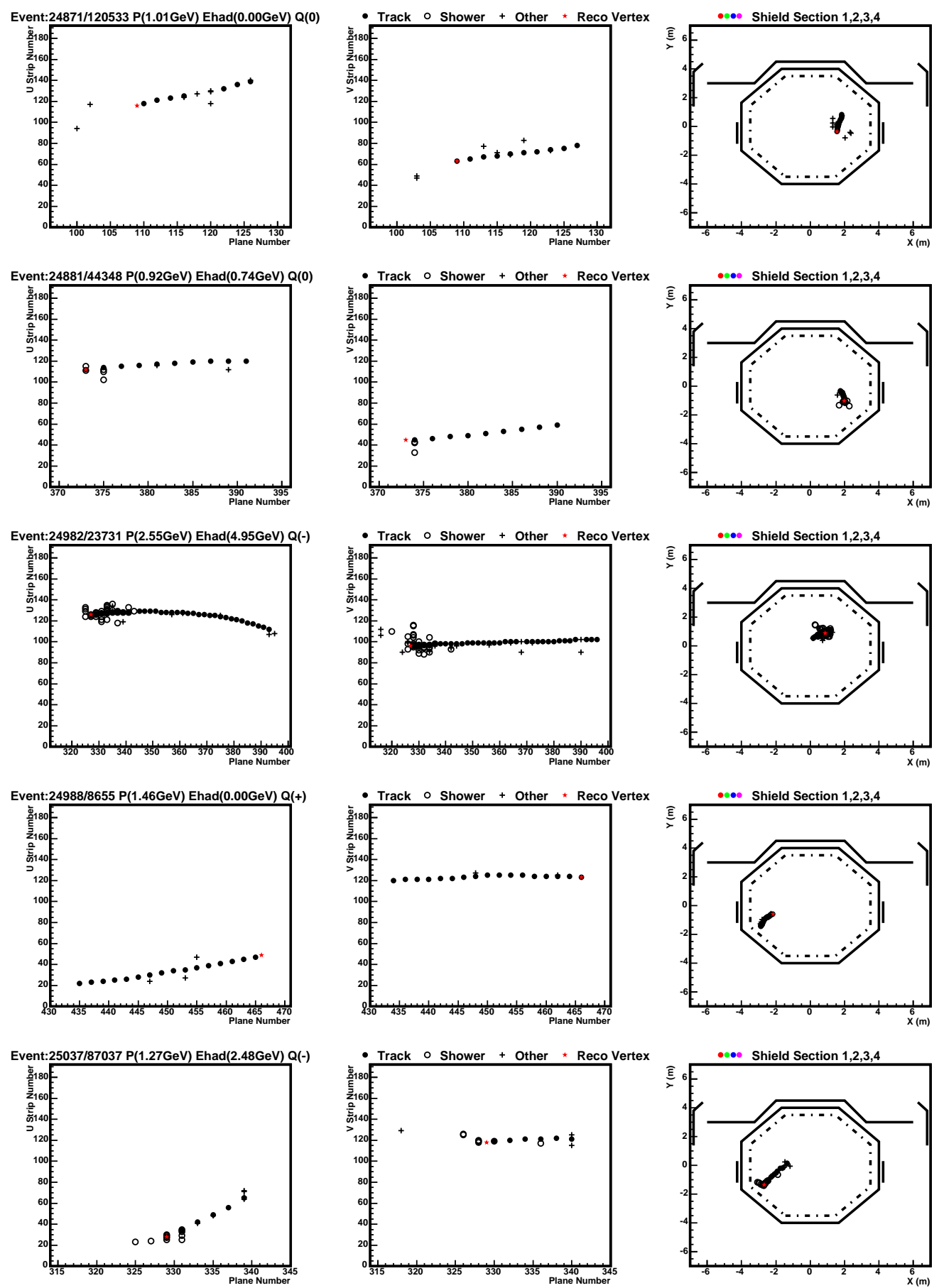


\section{Bibliography}

[1] J. Chadwick, Distribution in intensity in the magnetic spectrum of the $\beta$-rays of radium, Ver. Dtsch. Physik. Ges. 16 (1914) 383-391.

[2] J. Chadwick, Possible existence of a neutron, Nature 129 (1932) 312.

[3] E. Fermi, An attempt of a theory of beta radiation, Z. Phys. 88 (1934) 161-177.

[4] G. Danby et al., Observation of high-energy neutrino reactions and the existence of two kinds of neutrinos, Phys. Rev. Lett. 9 (1962) 36-44.

[5] DONUT Collaboration, K. Kodama et al., Observation of tau-neutrino interactions, Phys. Lett. B504 (2001) 218-224.

[6] S. Eidelman et al., Review of particle physics, Phys. Lett. B592 (2004) 1. http://pdg.lbl.gov.

[7] B. Pontecorvo, Inverse beta processes and nonconservation of lepton charge, Sov. Phys. JETP 7 (1958) 172-173.

[8] B. Pontecorvo, Mesonium and antimesonium, Sov. Phys. JETP 6 (1957) 429.

[9] J. Davis, Raymond, D. S. Harmer and K. C. Hoffman, Search for neutrinos from the sun, Phys. Rev. Lett. 20 (1968) 1205-1209.

[10] Super-Kamiokande Collaboration, Y. Fukuda et al., Measurement of a small atmospheric $\nu_{\mu} / \nu_{e}$ ratio, Phys. Lett. B433 (1998) 9-18.

[11] Z. Maki, M. Nakagawa and S. Sakata, Remarks on the unified model of elementary particles, Prog. Theor. Phys. 28 (1962) 870.

[12] L. Chau and W. Keung, Comments on the parametrization of the Kobayashi-Maskawa matrix, Phys. Rev. Lett. 53 (1984) 1802. 
[13] M. Apollonio et al., Search for neutrino oscillations on a long base-line at the CHOOZ nuclear power station, Eur. Phys. J. C27 (2003) 331-374.

[14] Palo Verde Collaboration, A. Piepke, Final results from the Palo Verde neutrino oscillation experiment, Prog. Part. Nucl. Phys. 48 (2002) 113-121.

[15] J. N. Bahcall, M. H. Pinsonneault and S. Basu, Solar models: Current epoch and time dependences, neutrinos, and helioseismological properties, Astrophys. J. 555 (2001) 990-1012.

[16] M. Thomson, Neutrino oscillations, Phil. Trans. Roy. Soc. Lond. A360 (2002) 921-938.

[17] B. T. Cleveland et al., Measurement of the solar electron neutrino flux with the Homestake chlorine detector, Astrophys. J. 496 (1998) 505-526.

[18] SAGE Collaboration, J. N. Abdurashitov et al., Measurement of the solar neutrino capture rate in SAGE, Nucl. Phys. Proc. Suppl. 118 (2003) 39-46.

[19] GNO Collaboration, T. A. Kirsten, Progress in GNO, Nucl. Phys. Proc. Suppl. 118 (2003) 33-38.

[20] Super-Kamiokande Collaboration, S. Fukuda et al., Solar ${ }^{8} \mathrm{~B}$ and hep neutrino measurements from 1258 days of Super-Kamiokande data, Phys. Rev. Lett. 86 (2001) 5651-5655.

[21] SNO Collaboration, Q. R. Ahmad et al., Measurement of the charged current interactions produced by ${ }^{8} \mathrm{~B}$ solar neutrinos at the Sudbury Neutrino Observatory, Phys. Rev. Lett. 87 (2001) 071301.

[22] SNO Collaboration, A. W. P. Poon, Recent results from the Sudbury Neutrino Observatory, Eur. Phys. J. C33 (2004) 823-825.

[23] KamLAND Collaboration, T. Araki et al., Measurement of neutrino oscillation with KamLAND: Evidence of spectral distortion, hep-ex/0406035.

[24] IMB Collaboration, R. Becker-Szendy et al., Neutrino measurements with the IMB detector, Nucl. Phys. Proc. Suppl. 38 (1995) 331-336.

[25] Kamiokande Collaboration, Y. Fukuda et al., Atmospheric muon-neutrino / electron-neutrino ratio in the multi-GeV energy range, Phys. Lett. B335 (1994) $237-245$. 
[26] Frejus. Collaboration, K. Daum, Determination of the atmospheric neutrino spectra with the Frejus detector, Z. Phys. C66 (1995) 417-428.

[27] The NUSEX Collaboration, M. Aglietta et al., Experimental study of atmospheric neutrino flux in the NUSEX experiment, Europhys. Lett. 8 (1989) 611-614.

[28] Super-Kamiokande Collaboration, M. Ishitsuka, Super-Kamiokande results: Atmospheric and solar neutrinos, hep-ex/0406076.

[29] Soudan 2 Collaboration, M. Sanchez et al., Observation of atmospheric neutrino oscillations in Soudan 2, Phys. Rev. D68 (2003) 113004.

[30] MACRO Collaboration, M. Ambrosio et al., Measurements of atmospheric muon neutrino oscillations, global analysis of the data collected with MACRO detector, Eur. Phys. J. C36 (2004) 323-339.

[31] Super-Kamiokande Collaboration, Y. Ashie et al., Evidence for an oscillatory signature in atmospheric neutrino oscillation, Phys. Rev. Lett. 93 (2004) 101801.

[32] V. D. Barger, J. G. Learned, S. Pakvasa and T. J. Weiler, Neutrino decay as an explanation of atmospheric neutrino observations, Phys. Rev. Lett. $\mathbf{8 2}$ (1999) 2640-2643.

[33] E. Lisi, A. Marrone and D. Montanino, Probing possible decoherence effects in atmospheric neutrino oscillations, Phys. Rev. Lett. 85 (2000) 1166-1169 hep-ph/0002053.

[34] K2K Collaboration, E. Aliu et al., Evidence for muon neutrino oscillation in an accelerator-based experiment, hep-ex/0411038.

[35] K2K Collaboration, T. Ishii, Recent K2K results, hep-ex/0406055.

[36] OPERA Collaboration, D. Duchesneau, The CERN-Gran Sasso neutrino program, eConf C0209101 (2002) TH09 hep-ex/0209082.

[37] LSND Collaboration, G. B. Mills, Neutrino oscillation results from LSND, Nucl. Phys. Proc. Suppl. 66 (1998) 382-386.

[38] A. Strumia, Interpreting the LSND anomaly: Sterile neutrinos or CPTviolation or...?, Phys. Lett. B539 (2002) 91-101. 
[39] M. C. Gonzalez-Garcia, M. Maltoni and T. Schwetz, Status of the CPT violating interpretations of the LSND signal, Phys. Rev. D68 (2003) 053007.

[40] KARMEN Collaboration, B. Armbruster et al., Upper limits for neutrino oscillations $\bar{\nu}_{\mu} \rightarrow \bar{\nu}_{e}$ from muon decay at rest, Phys. Rev. D65 (2002) 112001.

[41] V. Valuev, Search for $\nu_{\mu} \rightarrow \nu_{e}$ oscillations in the NOMAD experiment, Prepared for International Europhysics Conference on High- Energy Physics (HEP 2001), Budapest, Hungary, 12-18 Jul 2001.

[42] MiniBooNE Collaboration, P. Kasper, MiniBooNE overview and status, Pramana 62 (2004) 611-614.

[43] S. Palomares-Ruiz and S. T. Petcov, Three-neutrino oscillations of atmospheric neutrinos, $\theta_{13}$, neutrino mass hierarchy and iron magnetized detectors, hep-ph/0406096.

[44] CDMS Collaboration, L. Baudis, Search for cosmic dark matter at CDMS, ECONF C020805 (2002) TF02.

[45] Soudan-2 Collaboration, T. Kafka, The Soudan-2 experiment, Nucl. Phys. Proc. Suppl. 28A (1992) 377-381.

[46] A. Cabrera, Pedestal and Sparsification studies for the FarDet FEE: Truncation and Stability, 2003. MINOS-NuMI Internal Note NuMI-ANA-0999.

[47] P. Adamson et al., On the linearity of the minos light-injection calibration system, Nucl. Instrum. Meth. A521 (2004) 361-366.

[48] C. Bower, MINOS Far Detector Gamma Background and Rate Prediction, 1999. MINOS-NuMI Internal Note NuMI-L-569.

[49] J. Liu et al., Test of Anomalous PMT Singles Rates due to MINOS Fibers, 2003. MINOS-NuMI Internal Note NuMI-L-941.

[50] J. Hartnell, PMT Gain calibration for the MINOS Far Detector, Presented at MINOS Collaboration Meeting (FermiLab, Jan 2003).

[51] B. Rebel, Upward going neutrino induced muons in the MINOS Far Detector. $\mathrm{PhD}$ thesis, Indiana University, 2004.

[52] A. Belias et al., The MINOS Data Acquisition System, IEEE Trans. Nucl. Sci 51 (2004). 
[53] ROOT An Object-Oriented Data Analysis Framework, http://root.cern.ch/

[54] R. Halsall et al., MINOS DAQ Readout and Triggering, Conceptual Design Review, 2000. MINOS DAQ Note, http://www-numi.fnal.gov/minwork/daq/manuals/.

[55] M. A. Thomson, MINOS Run Control GUI, http://www-numi.fnal.gov/minwork/daq/manuals/.

[56] T. Nicholls, Data Acquisition Run Model, 2001. MINOS DAQ Note, http://www-numi.fnal.gov/minwork/daq/manuals/.

[57] E. Gamma, R. Helm, R. Johnson and J. Vlissides, Design Patterns: Elements of Reusable Object-Oriented Software. Addison-Wesley, 1995.

[58] F. Yergeau et al., Extensible Markup Language (XML) 1.0, 2004. W3C Recommendation, http://www.w3.org/TR/2004/REC-xml-20040204/.

[59] V. Agrawal, T. K. Gaisser, P. Lipari and T. Stanev, Atmospheric neutrino flux above 1 GeV, Phys. Rev. D53 (1996) 1314-1323.

[60] G. Battistoni, A. Ferrari, T. Montaruli and P. R. Sala, The FLUKA atmospheric neutrino flux calculation, Astropart. Phys. 19 (2003) 269-290.

[61] G. D. Barr et al., A three-dimensional calculation of atmospheric neutrinos, Phys. Rev. D70 (2004) 023006.

[62] M. Honda, T. Kajita, K. Kasahara and S. Midorikawa, A precise three-dimensional calculation of the atmospheric neutrino flux, Prepared for 28th International Cosmic Ray Conferences (ICRC 2003), Tsukuba, Japan, 31 Jul - 7 Aug 2003.

[63] D. J. Culter and D. E. Groom, Mayflower Mine 1500 GeV detector Cosmic-ray anisotropy and search for Cygnus X-3, Astrophys. J. 376 (1991) 322.

[64] T. K. Gaisser and M. Honda, Flux of atmospheric neutrinos, Ann. Rev. Nucl. Part. Sci. 52 (2002) 153-199. 
[65] E. S. Seo, Measurement of cosmic-ray proton and helium spectra during the 1987 solar minimum, Astrophys. J. 378 (1991) 763-772.

[66] WiZard/MASS2 Collaboration, R. Bellotti et al., Balloon measurements of cosmic ray muon spectra in the atmosphere along with those of primary protons and helium nuclei over mid-latitude, Phys. Rev. D60 (1999) 052002.

[67] M. Boezio et al., The Cosmic-Ray Proton and Helium Spectra between 0.4 and 200 GeV, Astrophys. J. 518 (1999) 457-472.

[68] IMAX Collaboration, W. Menn et al., The absolute flux of protons and helium at the top of the atmosphere using IMAX, Astrophys. J. 533 (2000) 281-297.

[69] BESS Collaboration, T. Sanuki et al., Precise measurement of cosmic-ray proton and helium spectra with the BESS spectrometer, Astrophys. J. $\mathbf{5 4 5}$ (2000) 1135-1142.

[70] M. Bruckel, The AMS experiment: Particle identification with the transition radiation detector, Surveys High Energy Phys. 18 (2003) 233-247.

[71] RUNJOB Collaboration, A. V. Apanasenko et al., Composition and energy spectra of cosmic-ray primaries in the energy range $10^{13}-10^{15} \mathrm{eV} /$ particle observed by Japanese-Russian joint balloon experiment, Astropart. Phys. 16 (2001) 13-46.

[72] Y. Kawamura et al., 'Quasidirect' observations of cosmic ray primaries in the energy region $10^{12} \mathrm{eV}$ to approximately $10^{14} \mathrm{eV}$, Phys. Rev. D40 (1989) 729.

[73] AGASA Collaboration, M. Takeda, Energy spectrum above $3 \times 10^{18} \mathrm{eV}$ observed with AGASA, Prepared for 26th International Cosmic Ray Conference (ICRC 99), Salt Lake City, Utah, 17-25 Aug 1999.

[74] Auger Collaboration, D. V. Camin, The Southern Pierre Auger Observatory: Status and prospectives, Nucl. Instrum. Meth. A518 (2004) 172-176.

[75] NASA Solar Physics, http://science.nasa.gov/ssl/pad/solar/sunspots.htm.

[76] M. C. Sanchez, Oscillation analysis of atmospheric neutrinos in Soudan 2. PhD thesis, Tufts University, 2003. UMI-30-98145. 
[77] H. Gallagher, The NEUGEN neutrino event generator, Nucl. Phys. Proc. Suppl. 112 (2002) 188-194.

[78] H. Gallagher. Private communication.

[79] A. Rubbia, NUX - neutrino generator, Presented at NuInt01, http://neutrino.kek.jp/nuint01/.

[80] D. Casper, The NUANCE neutrino physics simulation, and the future, Nucl. Phys. Proc. Suppl. 112 (2002) 161-170.

[81] G. P. Zeller, Low energy neutrino cross sections: Comparison of various Monte Carlo predictions to experimental data, hep-ex/0312061.

[82] T. Gaisser, Cosmic Rays and Particle Physics. Cambridge University Press, 1990.

[83] Soudan 2 Collaboration, S. M. Kasahara, A study of cosmic ray composition in the knee region using multiple muon events in the Soudan 2 detector, Prepared for 9th Annual Divisional Meeting (DPF 96) of the Division of Particles and Fields of the American Physical Society, Minneapolis, Minnesota, 11-15 Aug 1996.

[84] The GEANT detector description and simulation tool, CERN Program Library Long Writeup W5013.

[85] N. Tagg, DetSim and PhotonTransport: MINOS Detector and Front-End Simulation,

http://www-numi.fnal.gov/offline_software/srt_public_context/ WebDocs/detsim/DetSim_Documentation.html.

[86] The GCALOR program, http://www.physik.uni-mainz.de/zeitnitz/gcalor/gcalor.html.

[87] A. Fasso et al., The FLUKA code: Present applications and future developments, ECONF C0303241 (2003) MOMT004 [physics/0306162].

[88] R. Cassell and G. Bower. Private communication.

[89] M. A. Thomson, Alternative Demultiplexer: AltDemux, Presented at MINOS Collaboration Meeting (FermiLab, June 2003), http://www.hep.phy.cam.ac.uk/ thomson/minos/talks/fnal03.pdf. 
[90] A. J. Blake, Event Reconstrunction in the MINOS Far Detector, 2004. NuMI-MINOS Internal Note ATM_NU-1064.

[91] C. P. Ward. Private Communication.

[92] M. Kordosky, Hadronic interactions in the MINOS detectors. PhD thesis, University of Texas at Austin, 2004.

[93] G. J. Feldman and R. D. Cousins, A Unified approach to the classical statistical analysis of small signals, Phys. Rev. D57 (1998) 3873-3889. 